\title{
Symbiont diversity and coral bleaching: An antioxidant view on thermal stress
}

\author{
Thomas Krüger
}

A thesis submitted to Victoria University of Wellington in fulfilment of the requirements for the degree of Doctor of Philosophy in Science

Victoria University of Wellington

2014 
"So let us not be blind to our differences, but let us also direct attention to our common interests and the means by which those differences can be resolved. And if we cannot end now our differences, at least we can help make the world safe for diversity. For, in the final analysis, our most basic common link is that we all inhabit this small planet. We all breathe the same air. We all cherish our children's futures. And we are all mortal."

John Fitzgerald Kennedy

A Strategy of Peace

June 10, 1963 


\section{Abstract}

The functioning of coral reef systems, as biodiversity hotspots, is largely dependent on the symbiotic association between dinoflagellate symbionts (Symbiodinium spp.) and scleractinian coral hosts. The breakdown of this symbiosis (coral bleaching), as a result of global warming and other stressors, therefore has profound implications for the tropical marine environment. Corals associate with a variety of Symbiodinium genotypes, and it is this mosaic nature that contributes to the variable stress thresholds of corals. Research over the past 25 years has established that the generation and scavenging of reactive oxygen species (ROS) in both partners, under light and thermal stress, is a fundamental element of the bleaching response. However, while the existence of more thermally susceptible and tolerant symbiont types has been recognized, the differences in the antioxidant systems that may accompany these properties have received less attention. The purpose of this thesis was to explore the role of the antioxidant network in explaining the different thermal susceptibilities of various symbiont types and how the activity of key antioxidants in both partners under thermal stress relates to bleaching patterns in different corals. Thus, the specific objectives were to: (1) assess the antioxidant network response in different Symbiodinium types; (2) investigate the activity and structural diversity of key enzymatic antioxidants in different Symbiodinium types; (3) examine the regulation of these antioxidants at the transcriptomic and proteomic levels; and (4) contrast the symbiont's and host's antioxidant responses under bleaching conditions. Symbiodinium types in culture were found to differ significantly with regards to the concentration and activity of specific antioxidants, exhibiting magnitude scale differences in some of them. However, the response of the main removal pathway, involving superoxide dismutase (SOD) and ascorbate peroxidase (APX), under lethal thermal stress was fairly similar. Instead, the typespecific differences were found to lie in more downstream systems, and particularly in those associated with the maintenance of the glutathione redox state. A declining glutathione redox state was the common feature of the three thermally susceptible Symbiodinium types: B1, C1, and E. Indeed, in comparison to the most sensitive type (B1), the tolerant type F1 exhibited stronger antioxidant up-regulation and the successful preservation of the highly reduced glutathione 
pool. Comparing antioxidant gene orthologues from members of different Symbiodinium clades $(A-E)$ revealed a higher degree of sequence variation at the amino acid level for peroxidases, which reflected the genetic radiation of the genus. In contrast, primary defences in the form of SOD isoforms were highly conserved. Sequence variations between Symbiodinium types involved residues that constitute binding sites of substrates and co-factors, and therefore likely affect the catalytic properties of these enzymes. While expression of antioxidant genes was successfully measured in Symbiodinium B1, it was not possible to assess the link between transcriptomic expression and proteomic activity due to high variability in expression between replicates, and little response in their enzymatic activity over three days. In contrast to previous findings, up-regulation of antioxidant defences was not evident in Symbiodinium cells inside the host (i.e. in hospite). In fact, oxidative stress in the thermally sensitive corals Acropora millepora and Pocillopora damicornis was only apparent from increased host catalase activity, which interestingly preceded photosynthetic dysfunction of their symbionts. Baseline antioxidant activities of thermally tolerant and susceptible host species showed no differences, though the scavenging activities of the hosts were considerably higher than those of the symbionts. Baseline activities for the symbionts were different, however, with Symbiodinium $\mathrm{C} 15$ from the thermally tolerant coral Montipora digitata exhibiting the lowest activities for SOD and catalase peroxidase. This thesis provides significant findings with respect to the variability in antioxidant activity, structure, and network response in different Symbiodinium types in culture, and how these relate to thermal tolerance. What effect these differences have on the response in the intact symbiosis remains unclear, however, as the findings contradict the classic bleaching model of photoinhibition and symbiont-derived ROS. I argue, using previously published data, that heating rates might profoundly affect the way we perceive the antioxidant response of both partners to thermal stress, and that host antioxidant defences might not be as easily overwhelmed by symbiont ROS as suggested previously. This thesis reports important findings on the antioxidant system in different Symbiodinium types, but also raises new questions about the antioxidant response of the intact coral. 


\section{Contributions and publications}

This thesis is written as a series of stand-alone manuscripts, which are in preparation for submission. The author conducted all aspects of the experimental design, laboratory work, fieldwork, statistical analyses and writing, with assistance as described below.

\section{Chapter 2}

Krueger T., Becker S., Pontasch S., Dove S., Hoegh-Guldberg O., Leggat, W., Fisher P.L. and Davy S.K. (In review). Antioxidant plasticity and thermal sensitivity in the coral endosymbiont Symbiodinium sp. Journal of Phycology.

S.K. Davy and P.L. Fisher advised on experimental design, interpretation, analyses and writing. S. Becker, S. Pontasch, S. Dove, O. Hoegh-Guldberg, W. Leggat advised on writing. Assistance with laboratory work came from S. Becker (sampling, photosynthetic pigment analysis, cell counts) and P.L. Fisher (setup and sampling).

\section{Chapter 3}

Krueger T., Becker S., Pontasch S., Dove S., Hoegh-Guldberg O., Leggat, W., Fisher P.L. and Davy S.K. (In prep). Transcriptomic and proteomic characterization of enzymatic antioxidants in the dinoflagellate genus Symbiodinium. The Protist.

S.K. Davy and P.L. Fisher advised on experimental design, interpretation, analyses and writing. Assistance with the laboratory work came from P.L Fisher (primer design and preliminary findings for Symbiodinium C1) and S. Becker (exploratory PCRs). 


\section{Chapter 4}

Krueger T., Hawkins T. D., Becker S., Pontasch S., Dove S., Hoegh-Guldberg O., Leggat, W., Fisher P.L. and Davy S.K. (In prep). Differential antioxidant activity in the coral holobiont under thermal stress. Coral Reefs.

S.K. Davy and P.L. Fisher advised on experimental design, interpretation, analyses and writing. T.D. Hawkins and S. Becker advised on writing. Assistance with fieldwork came from T.D. Hawkins, P.L. Fisher and S.K. Davy. Assistance with laboratory work came from T.D. Hawkins (cell counts), S. Becker (photosynthetic pigment analysis, cell counts) and P.L. Fisher (coral surface determination). 


\section{Acknowledgements}

I would like to thank my supervisor Simon Davy for giving me the opportunity to pursue this $\mathrm{PhD}$ in his work group and allowing me, to work on something, I find very intriguing. While this $\mathrm{PhD}$ has been difficult and disappointing at times, Simon has encouraged me, and supported my work all the way to the end. I am grateful for his guidance in this part of my career. Having Paul Fisher as my cosupervisor, was the best thing that could have happened to me. Not only did he express a practical ingenuity that came in handy in a number of problems, he also had the wise foresight to bring my somewhat "grand" experimental designs to a manageable format. Over the course of the last 3.5 years, I had the luck to meet a number of people, who knowingly or unsuspectingly supported and inspired part of my work. It is also these people, who made some bad days more bearable, and everything worth the effort. For all the fantastic talks, exchange of ideas, distractions, funny moments, words of encouragement, and hugs, I thank my fellow lab researchers Paul, Stefanie, Tom, Jennifer, Anne, Emma, Shaun, Katie, Scott, Dorota, Mareike, Alyssa and Ben. A special thanks goes to Jennifer for taking care of getting my thesis submitted. I literally could not have done it without you!

With all the ups and downs of a PhD, I was grateful for the support and help of my wonderful partner, Susanne. Things would have been different without you, and you were a constant reminder that there is a world beyond the lab.

Having a natural curiosity is a good starting point for a scientist, but it needs an environment, in which this curiosity can grow into something useful. Getting to this point in my life was made possible by my parents, and I am grateful for their support along the way.

For all their help and support with administrative issues, and visa and enrolment matters at the beginning, and throughout my studies at VUW, I would like to express my gratitude to Rachel Zhang, Patricia Stein and Mary Murray.

For the original supply of the Symbiodinium cultures for my lab experiments, I would like to thank Scott Santos (Auburn University, Auburn, AL, USA), Mary Alice Coffroth (State University of New York at Buffalo, Buffalo, NY, USA), Gisele 
Muller-Parker (former Western Washington University, WA, USA) and the National Centre for Marine algae and Microbiota (NCMA [formerly CCMP], East Boothbay, MI, USA).

With regard to my field experiments, I wish to thank the staff at Heron Island Research Station (HIRS), University of Queensland. In particular, Kyra Hay and Liz Perkins deserve my gratitude for assistance with the fieldwork. I would also like to thank Olga Pantos, University of Queensland, for her help with the logistics of bringing part of the samples to Wellington.

Today's research is largely dependent on financial funds and my work was no exception. Therefore, I would like to acknowledge the financial support that was provided through a Coral Reef Biology PhD scholarship from the Royal Society of New Zealand Marsden Fund, a JL Stewart scholarship and a grant from the Botanical Society of Wellington. 


\section{List of figures}

Figure 1.1. Socio-economic dependence on coral reefs................................. 1 -

Figure 1.2. Histological schematic of zooxanthellate coral tissue. .................... 4 -

Figure 1.3. Conceptual framework of the coral stress response...................... 7 -

Figure 1.4. Differential coral bleaching...................................................... 8 -

Figure 1.5. The Oxidative Theory of Coral Bleaching. ................................. 12 -

Figure 1.6. Antioxidant network in chloroplasts............................................. 14 -

Figure 1.7. Foyer-Halliwell-Asada cycle..................................................... 16 -

Figure 1.8. Mitochondrial apoptosis pathway............................................ 20 -

Figure 2.1. Components of the antioxidant network assessed in this study....-28 -

Figure 2.2. Experimental setup........................................................... 30 -

Figure 2.3. Viability of Symbiodinium types B1, F1, C1 and E...................... 35 -

Figure 2.4. Type-specific antioxidant profiles in Symbiodinium....................... 37 -

Figure 2.5. SOD-APX pathway activity. ....................................................... 40 -

Figure 2.6. Size and redox state of the glutathione pool............................... 41 -

Figure 2.7. GR and GST activity in Symbiodinium B1 ................................ 42 -

Figure 2.8. PCA analysis of antioxidant responses.................................... 45 -

Figure 3.1. Protein alignment of Symbiodinium manganese superoxide

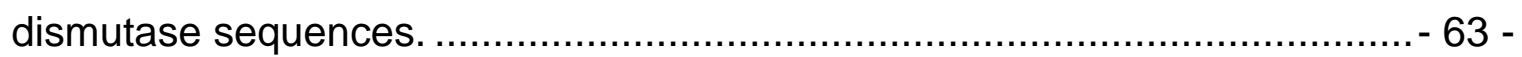

Figure 3.2. Predicted monomer structures of Symbiodinium manganese superoxide dismutase isoforms................................................................... 63 -

Figure 3.3. Protein phylogeny of manganese superoxide dismutase.............. 64 -

Figure 3.4. Alignment of iron superoxide dismutase protein sequences.........- 65 -

Figure 3.5. Alignment of ascorbate peroxidase protein sequences.................. 66 -

Figure 3.6. Ascorbate peroxidase protein phylogeny.................................. 68 -

Figure 3.7. Catalase peroxidase protein alignment..................................... 70 -

Figure 3.8. Structural comparison of the catalase peroxidase from the bacterium Haloarcula marismortui and Symbiodinium B1......................................... 71 -

Figure 3.9. Catalase peroxidase protein phylogeny................................... 72 -

Figure 3.10. Stability of HKG and gene expression in Symbiodinium B1........ 74 -

Figure 4.1. Experimental setup on Heron Island......................................... 84 -

Figure 4.2. Temperature profile of experimental system............................... 85 - 
Figure 4.3. Response of photobiological variables of three coral species under thermal stress. -90 -

Figure 4.4. Baseline activities of enzymatic antioxidants in host and symbiont of three different coral species. $91-$

Figure 4.5. Symbiont antioxidant activity................................................ - 93 -

Figure 4.6. Host antioxidant activity....................................................... - 94 -

Figure 5.1. Key antioxidant processes that define thermal thresholds in Symbiodinium at low light intensities. - 107 -

Figure 5.2. Schematic depiction of gradients for scavenging of hydrogen peroxide and superoxide in host and symbiont. - 114 -

Figure 7.1. Chorophyll a content per cell................................................ - 145 -

Figure 7.2. Protein content per cell. ................................................... - 146 -

Figure 7.3. Glutathione reductase activity. ............................................ - 147 -

Figure 7.4. Glutathione S-transferase activity. ………………………... - 148 -

Figure 7.5. Complete KatG alignment................................................. - 152 -

Figure 7.6. Melting curve analysis for genes of interest. ............................ - 156 -

Figure 7.7. Melting curve analysis for housekeeping genes....................... - 157 -

Figure 7.8. Calibration curves for genes of interest in Symbiodinium B1.... - 158 -

Figure 7.9. Calibration curves for potential housekeeping genes in Symbiodinium B1................................................................. - 159 -

Figure 7.10. Gel electrophoresis of PCR amplicons for suitable qPCR primers in Symbiodinium B1.................................................................... - 160 -

Figure 7.11. Symbiont density and chlorophyll a content........................... - 161 -

Figure 7.12. Ratios between total host and symbiont protein....................... - 162 -

Figure 7.13. Protein contents for host and symbiont................................ - 162 -

Figure 7.14. ITS2 rDNA signatures of isolated coral symbionts. .................. - 163 -

Figure 8.1. Acclimation treatments used for Symbiodinium B1 ................... - 166 -

Figure 8.2. Effects of thermal acclimation mode on physiological variables under thermal stress in Symbiodinium B1........................................................ - 168 - 


\section{List of tables}

Table 2.1. Baseline levels of antioxidants in Symbiodinium. $36-$

Table 2.2. Statistical effects of temperature and Symbiodinium type for

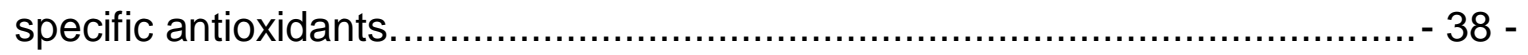

Table 3.1. Primer characteristics for gene expression of Symbiodinium B1 ... - 60 Table 3.2. Temperature effects on viability and physiological variables in Symbiodinium B1. -73 -

Table 4.1. Overall statistical analysis of photobiological variables. $-88-$

Table 4.2. Statistical analysis of physiological variables for each coral. - 95 -

Table 7.1 Distance matrices for pairwise comparison of amino acid sequences for different Symbiodinium ITS2 types. 153 -

Table 8.1. Statistical analysis for acclimation experiment. 167 
Abbreviations

$\Delta F / F_{m}^{\prime} \quad$ effective quantum yield of PSII

${ }^{1} \mathrm{O}_{2} \quad$ singlet oxygen

ANOVA analysis of variance

APAF1 apoptotic protease-

activating factor 1

APX

ascorbate peroxidase

ATP adenosine triphosphate

Bcl-2 B-cell lymphoma 2

BID

BIM

(caspase

CAT

cDNA copy deoxyribonucleic

acid

CDNB 1-chloro-2,4-

dinitrobenzene

Chl a chlorophyll a

Cl

confidence interval
$\mathrm{CO}_{2} \quad$ carbon dioxide

Cox cytochrome oxidase subunit 1

CuZnSOD copper zinc superoxide dismutase

D1 protein of photosystem

II reaction centre

DFA discriminant function

analysis

DIABLO direct IAP binding

protein with low $\mathrm{PI}$

DMF N, N-

dimethylformamide

DMS dimethylsulfide

DNA deoxyribonucleic acid

DTNB 5,5'-dithiobis(2-

nitrobenzoic acid)

EC-SOD extracellular CuZnSOD

EDTA ethylene diamide tetra

acetate

ER endoplasmic reticulum

EST expressed sequence

tag

Fd ferredoxin

FeSOD iron superoxide

dismutase 


\begin{tabular}{|c|c|c|c|}
\hline $\mathrm{F}_{\mathrm{m}}$ & maximum fluorescence & HNE & 4-hydroxynonenal \\
\hline \multirow[t]{2}{*}{ FNR } & ferredoxin-NADP ${ }^{+}$ & $\mathrm{HO}_{2}^{\bullet}$ & perhydroxyl radical \\
\hline & reductase & HSD & honestly significant \\
\hline$F_{v}$ & variable fluorescence & & difference \\
\hline \multirow[t]{2}{*}{$\mathrm{F}_{\mathrm{v}} / \mathrm{F}_{\mathrm{m}}$} & maximum quantum & HSP & heat shock protein \\
\hline & yield of photosystem II & IAP & inhibitors of apoptosis \\
\hline GFP & $\begin{array}{l}\text { green fluorescent } \\
\text { protein }\end{array}$ & ITS & $\begin{array}{l}\text { internal transcribed } \\
\text { spacer }\end{array}$ \\
\hline G-G & $\begin{array}{l}\text { Greenhouse-Geisser } \\
\text { correction }\end{array}$ & $\mathrm{K}_{2} \mathrm{HPO}_{4}$ & $\begin{array}{l}\text { dipotassium } \\
\text { monohydrogen }\end{array}$ \\
\hline GPX & glutathione peroxidase & & phosphate \\
\hline GR & glutathione reductase & KatG & catalase peroxidase \\
\hline \multirow[t]{3}{*}{$\mathrm{GSH} / \mathrm{GS} \mathrm{x}$} & $\begin{array}{l}\text { glutathione (reduced) } \\
\text { proportion of reduced }\end{array}$ & $\mathrm{KH}_{2} \mathrm{PO} 4$ & $\begin{array}{l}\text { potassium dihydrogen } \\
\text { phosphate }\end{array}$ \\
\hline & $\begin{array}{l}\text { glutathione relative to } \\
\text { total glutathione }\end{array}$ & LHC & $\begin{array}{l}\text { light harvesting } \\
\text { complex }\end{array}$ \\
\hline & content & M2VP & methyl-2- \\
\hline GSSG & glutathione (oxidized) & & vinylpyridinium \\
\hline GST & $\begin{array}{l}\text { glutathione S- } \\
\text { transferase }\end{array}$ & MAA & $\begin{array}{l}\text { mycosporine-like amino } \\
\text { acid }\end{array}$ \\
\hline GSx & $\begin{array}{l}\text { total glutathione } \\
\text { content }\end{array}$ & $\begin{array}{l}\text { MANOVA } \\
\text { MDA }\end{array}$ & $\begin{array}{l}\text { multivariate ANOVA } \\
\text { malondialdehyde }\end{array}$ \\
\hline $\mathrm{H}_{2} \mathrm{O}$ & water & MnSOD & manganese superoxide \\
\hline $\mathrm{H}_{2} \mathrm{O}_{2}$ & hydrogen peroxide & & dismutase \\
\hline HGT & horizontal gene transfer & & \\
\hline HKG & housekeeping gene & & \\
\hline
\end{tabular}




\begin{tabular}{|c|c|c|c|}
\hline MPTP & mitochondrial & PCD & programmed cell death \\
\hline & $\begin{array}{l}\text { membrane permeability } \\
\text { transition pores }\end{array}$ & PCR & $\begin{array}{l}\text { polymerase chain } \\
\text { reaction }\end{array}$ \\
\hline $\mathrm{NADP}^{+}$ & $\begin{array}{l}\text { nicotinamide adenine } \\
\text { dinucleotide phosphate }\end{array}$ & PSI & photosystem I \\
\hline NADPH & reduced nicotinamide & PSII & photosystem II \\
\hline & $\begin{array}{l}\text { adenine dinucleotide } \\
\text { phosphate }\end{array}$ & PUMA & $\begin{array}{l}\text { p53 upregulated } \\
\text { modulator of apoptosis }\end{array}$ \\
\hline NBT & nitroblue tetrazolium & $\mathrm{Q}_{\mathrm{m}}$ & $\begin{array}{l}\text { excitation pressure } \\
\text { across PSII }\end{array}$ \\
\hline NOS & nitric oxide synthase & qPCR & $\begin{array}{l}\text { quantitative polymerase } \\
\text { chain reaction }\end{array}$ \\
\hline$N P Q$ & $\begin{array}{l}\text { non-photochemical } \\
\text { quenching }\end{array}$ & $\mathrm{Rf}$ & riboflavin \\
\hline NR & nitric reductase & rDNA & ribosomal DNA \\
\hline $\mathrm{O}_{2}$ & molecular oxygen & rmANOVA & $\begin{array}{l}\text { repeated measures } \\
\text { ANOVA }\end{array}$ \\
\hline $\mathrm{O}_{2}{ }^{--}$ & superoxide radical & RNA & ribonucleic acid \\
\hline $\mathrm{OH}^{\bullet}$ & hydroxyl radical & $\mathrm{RNAi}$ & RNA interference \\
\hline $\mathrm{ONOO}^{-}$ & peroxynitrite & RNS & reactive nitrogen \\
\hline ORF & open reading frame & & species \\
\hline PAM & $\begin{array}{l}\text { pulse-amplitude- } \\
\text { modulation }\end{array}$ & $\begin{array}{l}\mathrm{ROOH} \\
\mathrm{ROS}\end{array}$ & $\begin{array}{l}\text { lipid hydroperoxide } \\
\text { reactive oxygen }\end{array}$ \\
\hline PAR & $\begin{array}{l}\text { photosynthetically } \\
\text { active radiation (400- } \\
700 \mathrm{~nm})\end{array}$ & RT & $\begin{array}{l}\text { species } \\
\text { room temperature }\end{array}$ \\
\hline PCA & $\begin{array}{l}\text { principal components } \\
\text { analysis }\end{array}$ & & \\
\hline
\end{tabular}




$\begin{array}{ll}\text { Rubisco } & \text { ribulose-1,5- } \\ \text { bisphosphate } & \text { carboxylase/ } \\ & \text { oxygenase }\end{array}$
SAM S-adenosyl methionine synthetase
SD standard deviation
SE standard error of mean
SMAC second mitochondrial activator of caspases
SOD superoxide dismutase
SST sea surface
temperature

SymKatG catalase peroxidases of members of the genus Symbiodinium

Tub B-tubulin

Tukey HSD Tukey's Honestly Significant Difference

U units

UV ultraviolet radiation 


\section{Table of contents}

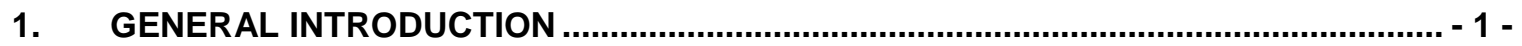

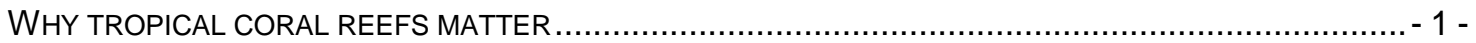

SYMBIOSIS AS THE FOUNDATION FOR TROPICAL CORAL REEF ECOSYSTEMS .................................. - 2 -

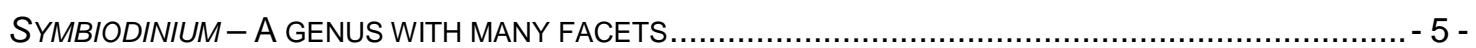

CORAL BLEACHING - THE SEARCH FOR A UNIFYING THEORY................................................... 6 -

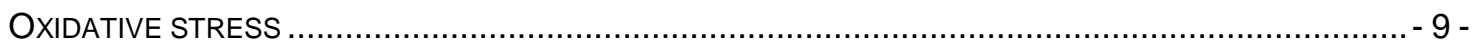

Photosynthesis under stress - ROS formation in the chloroplast ................................. - 10 -

Antioxidative enzyme mechanisms...................................................................... - 13 -

Low molecular weight non-enzymatic antioxidants ................................................. - 15 -

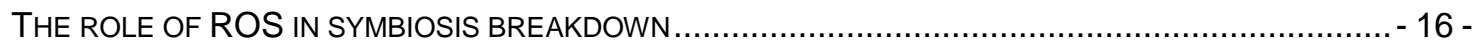

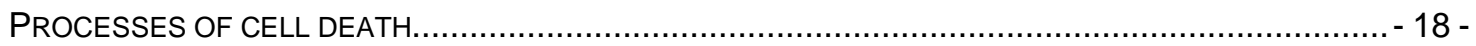

A potential role of calcium in the apoptotic cascade.................................................... - 21 -

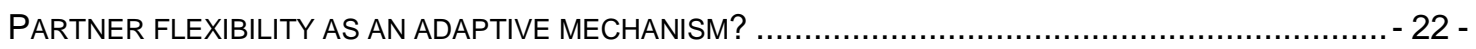

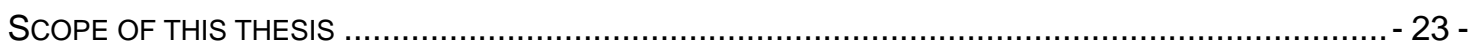

2. ANTIOXIDANT PLASTICITY AND THERMAL SENSITIVITY IN THE CORAL

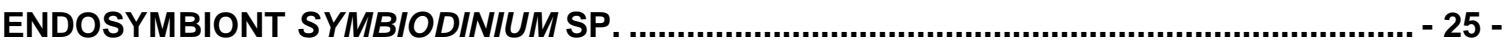

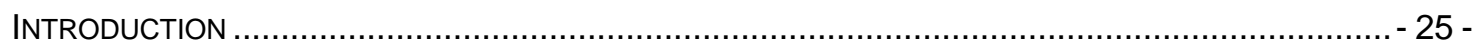

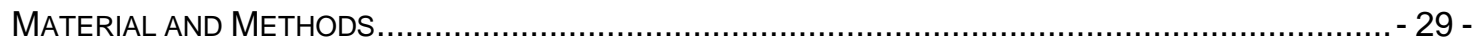

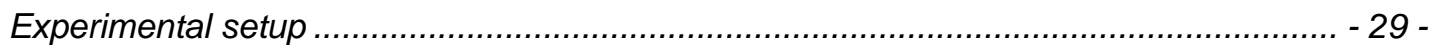

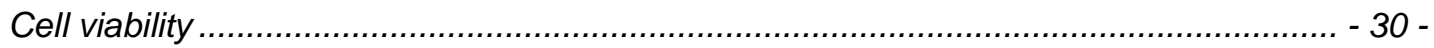

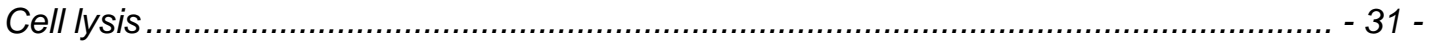

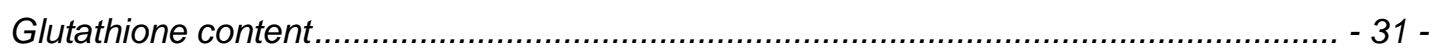

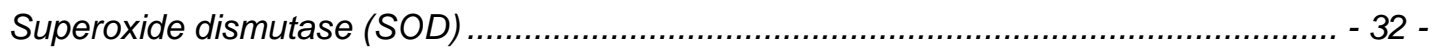

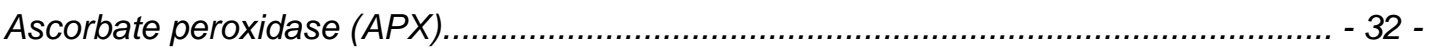

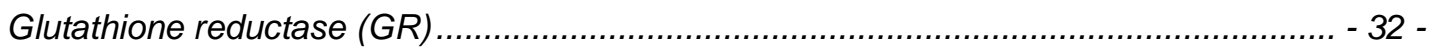

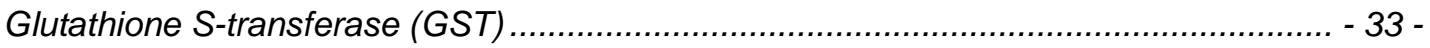

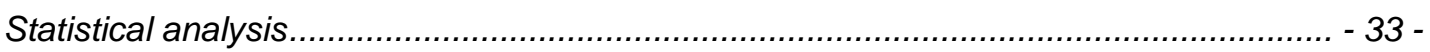

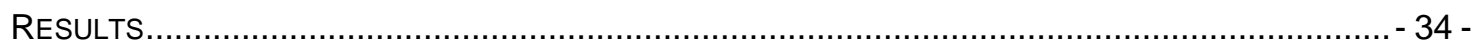

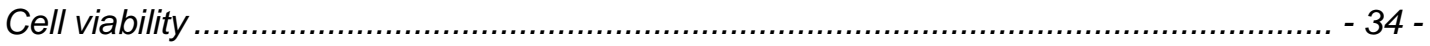

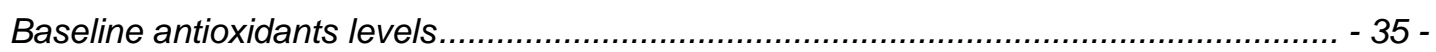

Antioxidant response in Symbiodinium B1 .......................................................... 38 -

Antioxidant response in Symbiodinium C1 ............................................................ - 42 -

Antioxidant response in Symbiodinium E ............................................................. - 43 -

Antioxidant response in Symbiodinium F1.......................................................... - 43 -

Network responses in Symbiodinium genotypes...................................................... - 44 -

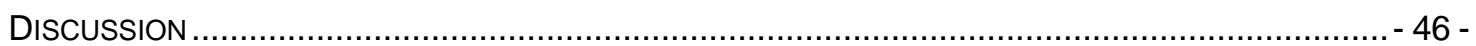

Antioxidant profiles are type specific in Symbiodinium ............................................. - 46 - 
Photosynthesis and the SOD-APX pathway $47-$

The glutathione system as an important part of the antioxidant network under thermal stress. $-48-$

Antioxidant plasticity as a key for acclimation $50-$

3. TRANSCRIPTOMIC AND PROTEOMIC CHARACTERIZATION OF ENZYMATIC ANTIOXIDANTS IN THE DINOFLAGELLATE GENUS SYMBIODINIUM ............................... 53 -

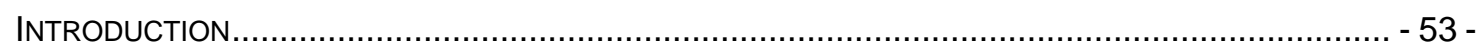

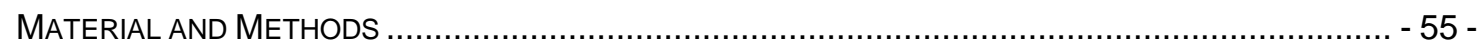

Sequence characterization and phylogenetic analysis of antioxidant genes ................... - 55 -

Symbiodinium types, RNA isolation, and cDNA generation ........................................................ 55 -

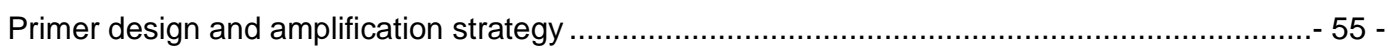

Identification, alignment and annotation of coding sequences.................................................... 56 -

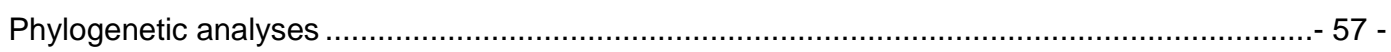

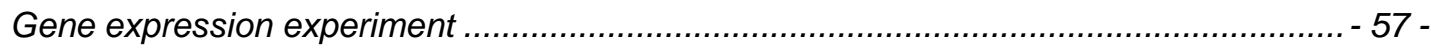

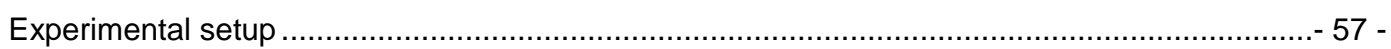

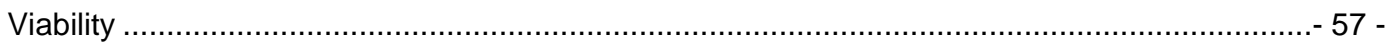

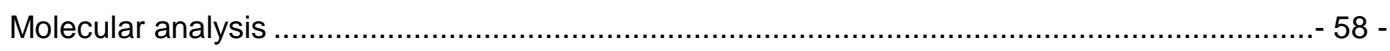

Primer design and amplification of housekeeping genes and genes of interest for

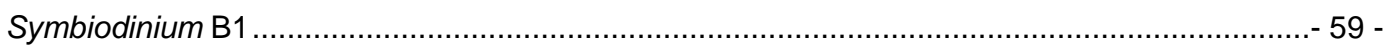

Quantitative polymerase chain reaction (qPCR) ………….................................................. 59 -

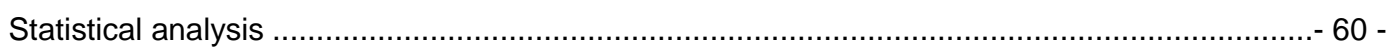

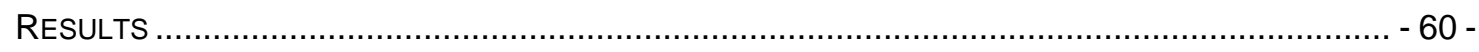

Molecular characterization of antioxidant genes in the genus Symbiodinium .................. 60 -

Manganese superoxide dismutase ................................................................................. 60 -

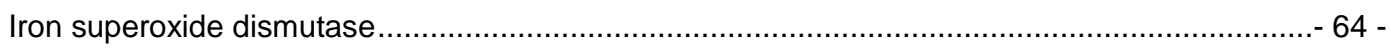

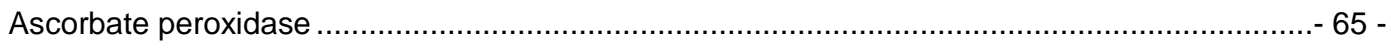

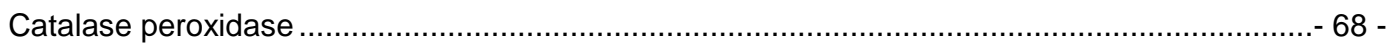

Viability and proteomic temperature response of Symbiodinium B1 ............................ - 72 -

Antioxidant gene expression in Symbiodinium B1 under thermal stress ........................ - 73 -

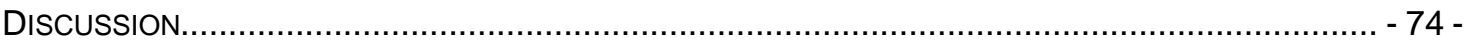

SOD isoforms are highly conserved between Symbiodinium types.............................. - 74 -

C-terminal reduction and additional loops characterize Symbiodinium catalase

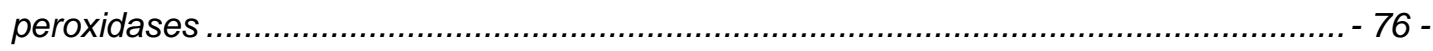

Sequence evolution of peroxidases reflect patterns of genetic radiation in the genus

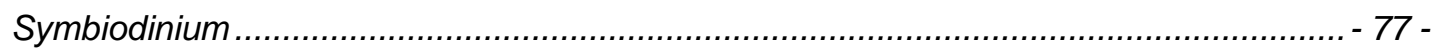

Antioxidant gene expression in Symbiodinium B1 …............................................ 78 -

4. DIFFERENTIAL ANTIOXIDANT ACTIVITY IN THE CORAL HOLOBIONT UNDER

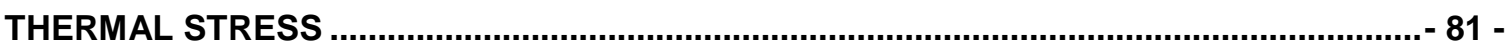

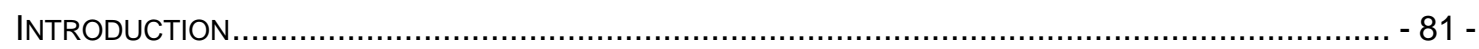

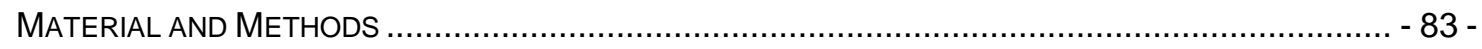




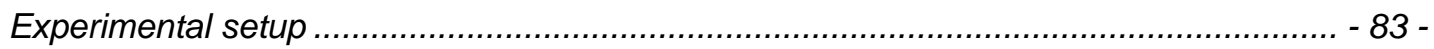

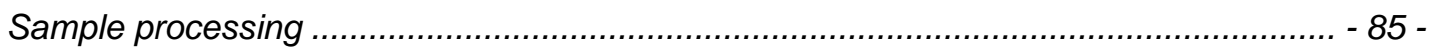

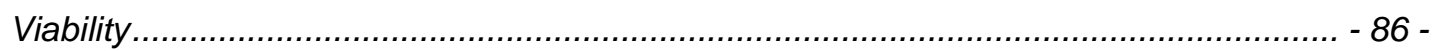

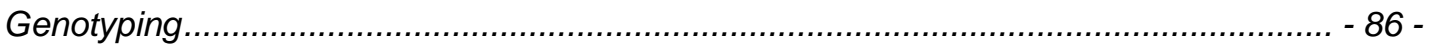

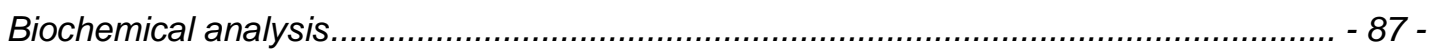

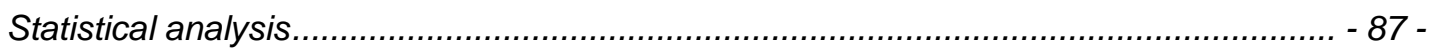

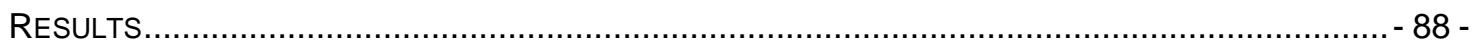

Symbiont identity, photobiological responses and bleaching ....................................... - 88 -

Antioxidant features ............................................................................................ 90 -

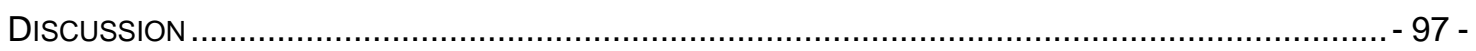

Photophysiological stress at high temperatures …................................................ - 97 -

Oxidative stress in host and symbiont as a temperature response ................................ - 98 -

ROS and coral bleaching - the role of hydrogen peroxide ........................................... - 100 -

Antioxidants and bleaching in corals - the role of the host ......................................... - 101 -

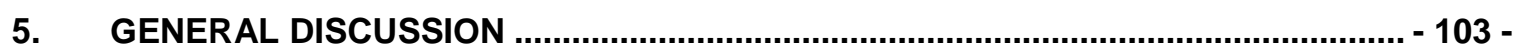

HOW SYMBIODINIUM GENOTYPES DIFFER IN THEIR ANTIOXIDANT PHYSIOLOGY.......................... - 105 -

IS GENETIC REDUNDANCY A DRIVER OF VARIABILITY IN ANTIOXIDANT DEFENCES DOWNSTREAM OF

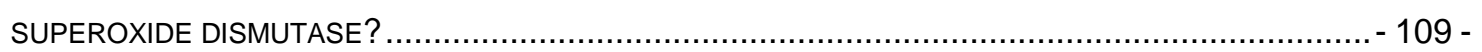

IS THERE A LINK BETWEEN HEATING RATE AND THE TEMPORAL ONSET OF ROS PRODUCTION IN BOTH

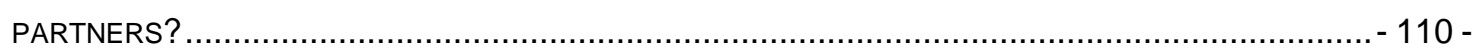

CAN THE SYMBIONT CHALLENGE THE HOST WITH ROS? ...................................................... 112 -

CELL BIOLOGY IS THE KEY TO UNDERSTANDING THE THERMAL RESPONSE OF CORALS................. - 114 -

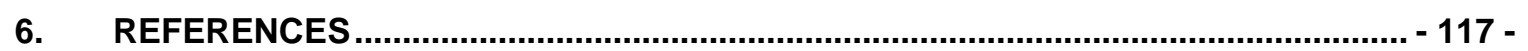

7. APPENDIX

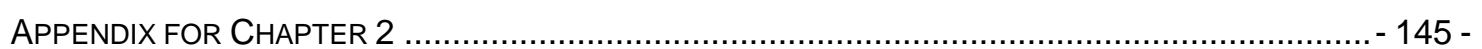

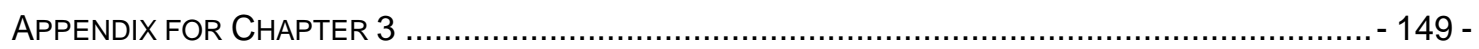

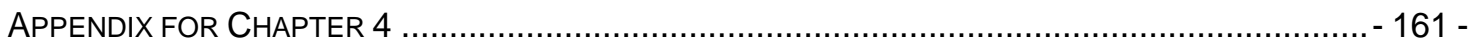

8. ADDITIONAL WORK 


\section{General Introduction}

\section{Why tropical coral reefs matter}

According to the United Nations Atlas of the Oceans, an estimated $44 \%$ of the world's population lives within 150 kilometres of the coast. Coastal ecosystems such as coral reefs therefore provide ecosystem goods and services for a large number of people. These fall into the categories of disturbance regulation, waste treatment, biological control, habitat/refugia, food production, raw materials, recreational services, cultural goods and storm protection (Martínez et al., 2007). Estimates for the total value and the benefits of ecosystem services of tropical coral reefs vary for different regions in the world, but a global annual net benefit of US $\$ 29.8$ billion has been suggested (reviewed in Conservation International, 2008). On the other hand, coral reefs together with sea-ice biomes have been identified as the two marine ecosystems that are "likely to be especially affected by climate change" (IPCC, 2007). Social and economic dependence on coral reefs is often very high for a number of oceanic nations, where large parts of the population depend on reef fisheries as a means of employment and nutrition (Figure 1.1, Burke et al., 2011).

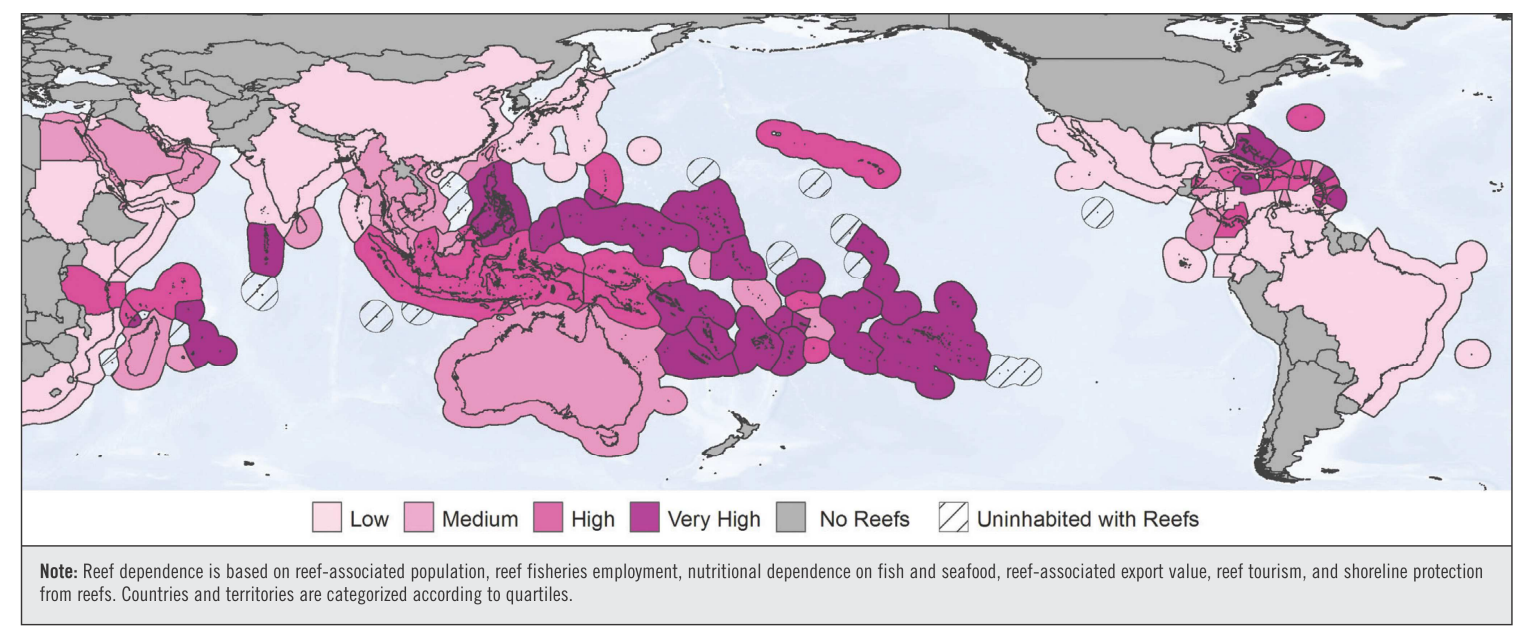

Figure 1.1. Socio-economic dependence on coral reefs.

Regional dependence on reef-based ecosystem goods and services for coastal populations differs between countries. Source: Burke et al. (2011).

In other Pacific regions such as Guam or Hawai'i, the recreational value of reefs generates a substantial proportion $(>75 \%)$ of annual tourism income (Cesar \& 
Van Beukering, 2004, van Beukering et al., 2007). Overuse of resources and negative impacts of increasing urbanization are therefore the main local threats for coral reefs today. In particular, overfishing and destructive fishing, coastal development and damage, watershed-based pollution and marine-based pollution are detrimental to the health of coral reef ecosystems (Burke et al., 2011). In addition to these local threats, increasing warming of the oceans and the increasing trend of acidification are considered major threats to a number of marine systems, including coral reefs (reviewed in Leadley, 2010).

\section{Symbiosis as the foundation for tropical coral reef ecosystems}

The main tropical reef-builders are stony corals (Scleractinia). Individual polyps of basic cnidarians (class: Anthozoa) deposit calcium carbonate, which provides the structural foundation of coral reefs. Shallow reef formations are built by zooxanthellate corals, i.e. corals that harbour symbiotic dinoflagellates of the genus Symbiodinium. In contrast to cold-water corals, which are predominantly azooxanthellate and restricted to water temperatures between $4^{\circ} \mathrm{C}$ and $12^{\circ} \mathrm{C}$ (Roberts et al., 2006), tropical shallow corals are latitudinally restricted to water temperatures of $18-30^{\circ} \mathrm{C}$ (roughly between $30^{\circ} \mathrm{N}$ and $30^{\circ} \mathrm{S}$ ) (Sheppard et al., 2009). Within this temperature range, calcification rates are usually positively correlated with water temperature (Lough \& Barnes, 2000, Silverman et al., 2007). However, distribution and growth of corals is also governed by light availability, $\mathrm{CaCO}_{3}$ saturation state, nutrient concentrations, salinity, hydrodynamic forces and the availability of hard substrate (Sheppard et al., 2009).

Corals are physical ecosystem engineers, defined as "organisms that directly or indirectly control the availability of resources to other organisms by causing physical state changes in biotic or abiotic materials" (Jones et al., 1994). They provide structural habitat but also retain and transfer energy to higher trophic levels, making the coral polyp a key component of reef function. The key to the success of tropical corals is their endosymbiotic dinoflagellates. This association is generally perceived as a mutualistic symbiosis, in which the symbiont provides photosynthetically-derived organic metabolites in exchange for inorganic waste products from the coral host (Muller-Parker \& D'Elia, 1997). 
Symbiodinium is known to associate with hosts, spanning across several taxa such as corals, anemones, jellyfish, tridacnid bivalves, sponges and foraminiferans (Trench, 1997, Pochon et al., 2001, Lobban et al., 2002, Stat et al., 2006, Hernawan, 2008). In corals, Symbiodinium cells in their non-motile coccoid state are enclosed in vacuoles within the host's endodermal cells (Figure 1.2). The host-symbiont interface is represented by the "symbiosome membrane complex" that consists of a number of algal- and host-derived membrane layers, where the final host vacuolar membrane is referred to as the "symbiosome membrane" (Wakefield \& Kempf, 2001, Kazandjian et al., 2008). The symbiosome membrane has been shown to contain ATPases and ATP-driven membrane transporters, which most likely provide the mechanism for active transmembrane transport of metabolites between both partners (Rands et al., 1993, Peng et al., 2010).

Translocated metabolites may include, but are not limited to, photosyntheticallyfixed carbon in the form of glycerol, glucose, lipids, neutral amino acids and some organic acids (reviewed in Hinde, 1988, Whitehead \& Douglas, 2003, Venn et al., 2008, Davy et al., 2012). In addition, due to their endosymbiotic nature, the dinoflagellate symbionts recycle inorganic waste from the host and assimilate it into organic compounds. Indeed, the assimilation of inorganic nitrogenous compounds into organic products is one of the key functions of these endosymbionts (Taguchi \& Kinzie III, 2001, Tanaka et al., 2006, Kopp et al., 2013) and the cyclical exchange and conservation of nutrients is an important factor that allows corals to grow in otherwise nutrient-poor waters (Muscatine \& Porter, 1977, Yellowlees et al., 2008). Moreover, the algal symbionts significantly enhance calcification rates in scleractinian corals (Pearse \& Muscatine, 1971, Gattuso et al., 1999). 


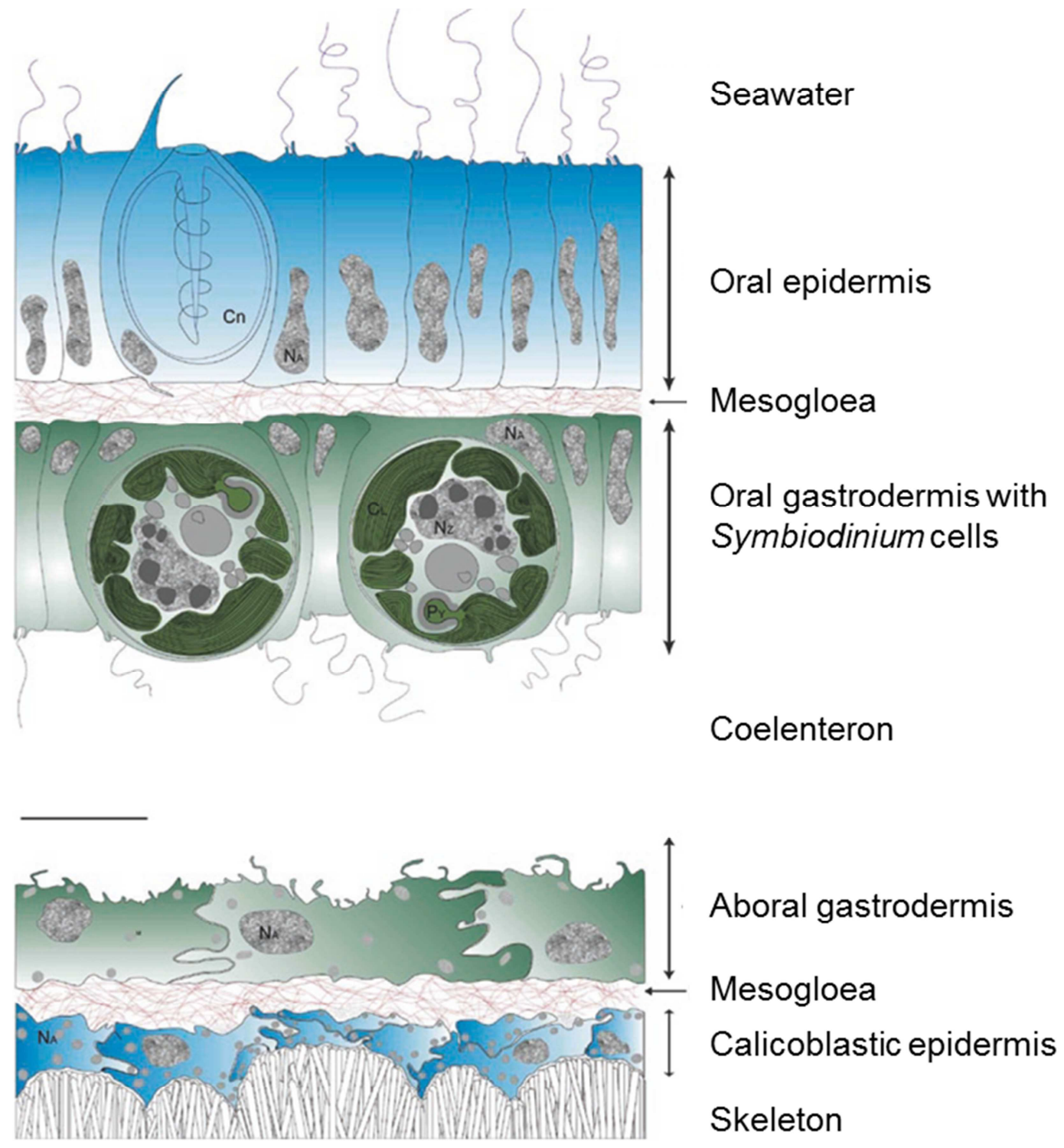

Figure 1.2. Histological schematic of zooxanthellate coral tissue.

Outer layers include oral epidermis with stinging cells (cnidocytes, $C_{n}$ ) and oral gastrodermis with enclosed Symbiodinium cells (chloroplast, $C_{L}$ and pyrenoid $P_{Y}$ ). Basal layers consist of the aboral gastrodermis adjacent to the calicoblastic epidermis, responsible for calcification. Scale bar length $=5 \mu \mathrm{m}$. Cell nuclei of animal $\left(\mathrm{N}_{\mathrm{A}}\right)$ or algae $\left(\mathrm{N}_{\mathrm{Z}}\right)$ are indicated; small grey spots inside of cells represent mitochondria. Source: adapted from Allemand et al. (2011).

Coral hosts can acquire their symbionts either through maternal inheritance (vertical transmission) or through uptake from the environment (horizontal transmission). The establishment of a stable symbiosis through horizontal transfer has been shown to be a complex process, which is not yet completely understood. One important recognition mechanism probably involves the interaction of symbiont cell-surface glycans with specific host lectins (Lin et al., 
2000, Wood-Charlson et al., 2006, Kvennefors et al., 2008, Logan et al., 2010). Symbiont cells are phagocytosed by the gastrodermal layer after ingestion (Reynolds et al., 2000, Schwarz \& Weis, 2003). The induction of their non-motile state is probably also induced through lectin interactions (Jimbo et al., 2000, Koike et al., 2004). In summary, specificity in the host-symbiont pairing is achieved by preferential uptake of "suitable" Symbiodinium types through the initial glycan-lectin interaction, but also by post-phagocytotic steps, termed "winnowing" (Nyholm \& McFall-Ngai, 2004, Dunn \& Weis, 2009).

\section{Symbiodinium - A genus with many facets}

In vitro cultivation of coral symbionts allowed their identification as gymnoid dinoflagellates and their description as a single species with the name Symbiodinium microadriaticum (Kawaguti, 1944, McLaughlin \& Zahl, 1957, McLaughlin \& Zahl, 1959, Freudenthal, 1962). However, early studies investigating cellular ultrastructure, cell morphology and physiological features suggested that the genus Symbiodinium might contain more than one species (Schoenberg \& Trench, 1980a, Schoenberg \& Trench, 1980b). Indeed, through the application of molecular tools and the use of ribosomal DNA sequences as a taxonomic marker, the genus Symbiodinium was revealed to contain a high degree of diversity (Rowan \& Powers, 1991b, LaJeunesse, 2001). To date, nine major Symbiodinium clades (A-I) with various subclades (types) within each clade have been identified (LaJeunesse, 2005, Pochon et al., 2006, Pochon \& Gates, 2010). Diversity at the subcladal level has been revealed through the use of faster-evolving internal transcribed spacer (ITS) regions (mainly ITS2), and confirmed by mitochondrial and chloroplastic markers (Santos et al., 2002, Takishita et al., 2003b, Takabayashi et al., 2004, Sampayo et al., 2009). This molecular phylogeny enabled the development of evolutionary models of genetic radiation and host specialization for the genus Symbiodinium (LaJeunesse, 2005, Pochon et al., 2006), which identified members of clade $C$ as the dominant symbionts of Indo-Pacific corals, and clade B and $C$ as the most common symbionts in the Caribbean region (Baker, 2003).

The molecular identification of symbiont types made it possible to investigate the link between the physiological features of different holobiont (i.e. coral and 
dinoflagellate combined) pairings and their respective Symbiodinium types. In vitro studies of cultures, as well as holobiont studies, have indicated variability between Symbiodinium types with respect to light intensity and temperature tolerance (e.g., Iglesias-Prieto \& Trench, 1994, Robison \& Warner, 2006, Suggett et al., 2008, Ragni et al., 2010). These differences are also evident in the existence of type zonation patterns within coral colonies and over depth gradients (Rowan \& Knowlton, 1995, van Oppen et al., 2001, Ulstrup \& van Oppen, 2003). Thus, specific host-Symbiodinium pairings are commonly correlated with temperature and irradiance (reviewed in Coffroth \& Santos, 2005), but can express flexibility during ontogenesis (Weis et al., 2001, Little et al., 2004). Consequently, the physiology of particular coral holobionts and their differential bleaching susceptibilities under stressful conditions are largely driven by the specific combination of one or multiple symbiont types and host species (Bhagooli \& Hidaka, 2003, Rowan, 2004b, Loram et al., 2007, Abrego et al., 2008, Sampayo et al., 2008, Cantin et al., 2009, Fitt et al., 2009).

\section{Coral bleaching - The search for a unifying theory}

Coral bleaching can be defined as visible whitening of the coral as a result of decreasing densities of the Symbiodinium cells and/or declines in photosynthetic pigments, and is regarded as a symptom for the breakdown of the symbiosis, which can lead to coral mortality (Iglesias-Prieto et al., 1992, Glynn, 1996, Brown, 1997). Localized coral bleaching as a result of reduced salinity, aerial exposure, excessive UV radiation or disease occurs on all reefs, and contributes to natural erosion and turnover among corals (Sheppard et al., 2009). Coral bleaching on large spatial scales is, however, a considerable threat for the existence of coral reefs. Global reef cover is in decline as a result of local pressures and increasing sea surface temperatures (SST) (Gardner et al., 2003, Hughes et al., 2003, Pandolfi et al., 2003, Bruno \& Selig, 2007, Carpenter et al., 2008). Mass coral bleaching events, which affect multiple species over large areas and multiple locations around the globe are linked to increasing SST (Glynn, 1993, Oliver et al., 2009). Most corals live near their upper thermal tolerance limits and this becomes critical during the seasonal maximum SST in summer, when temperatures can reach $28.2-34.4^{\circ} \mathrm{C}$ (Kleypas et al., 1999). However, individual 
bleaching thresholds of corals are also influenced by a number of other factors, such as duration and intensity of stress, the composition and ontogenetic state of the holobiont, and the thermal history of the coral (Figure 1.3).

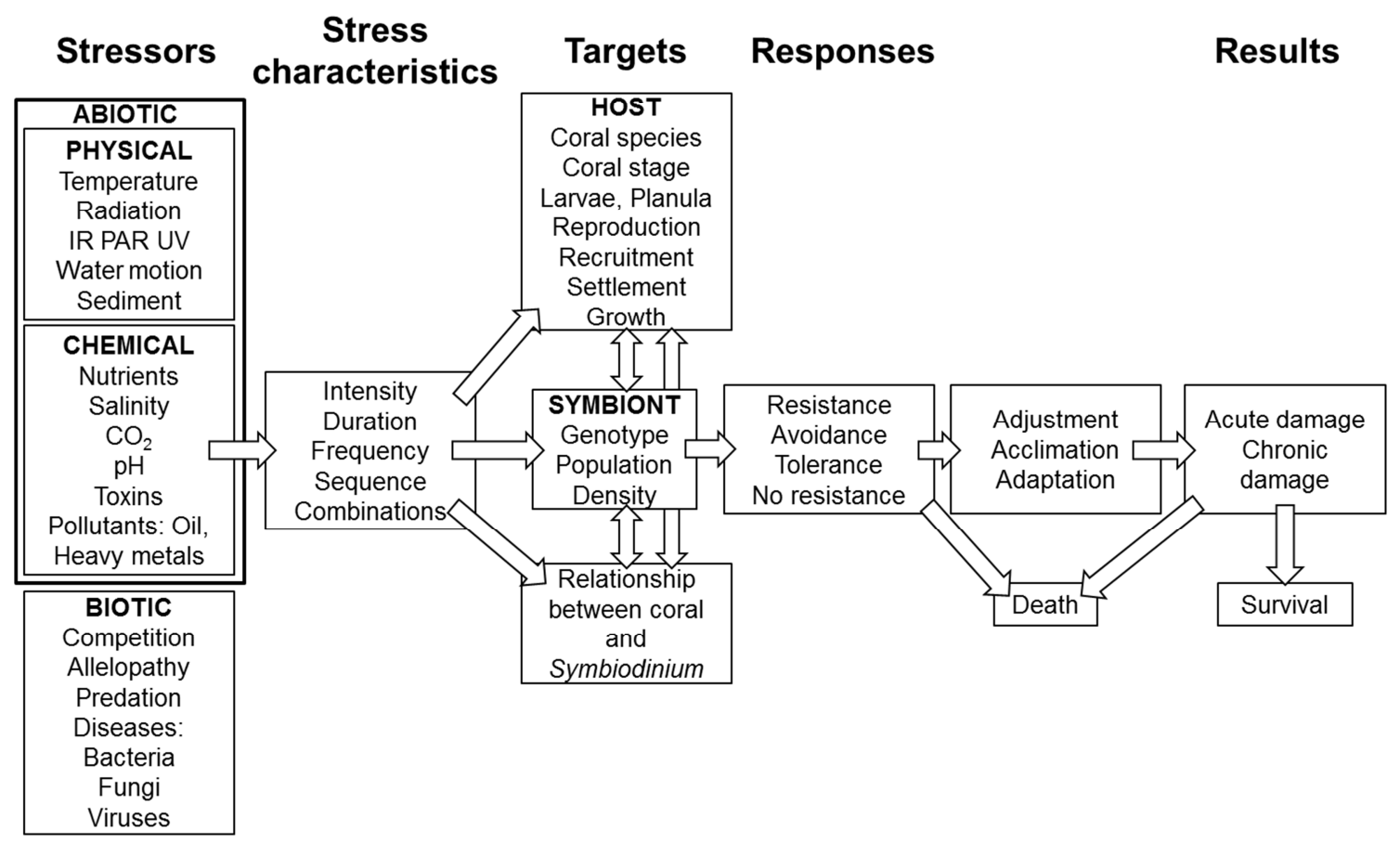

Figure 1.3. Conceptual framework of the coral stress response.

Overview of different factors and levels that influence coral stress responses and determine death or survival. Source: adapted from Stambler (2010).

As result of the combination of particular stress characteristics and the physiological state of the holobiont, thermal stress events do not affect all corals to the same extent, even when in spatial proximity (Figure 1.4). Frequent differential bleaching of coral communities as a result of environmental stress can lead to shifts in species composition and the overall structure of the coral reef over time (Berumen \& Pratchett, 2006, Golbuu et al., 2007, Pratchett et al., 2013). 

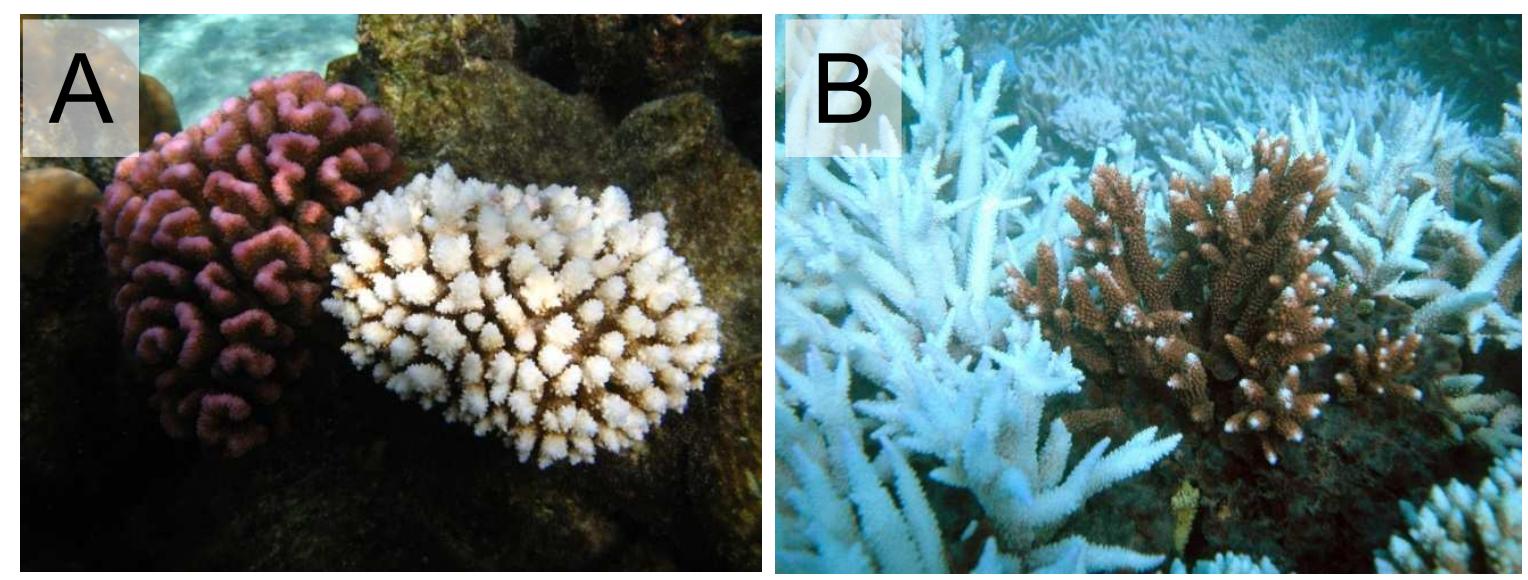

Figure 1.4. Differential coral bleaching.

Corals express different bleaching thresholds between species $(A)$, but also between conspecific hosts (B). Source: with permission of Morgan Pratchett, James Cook University ( $A$; bleached Acropora sp. adjacent to Pocillopora sp., Moorea, French Polynesia, 2007) and Ove Hoegh-Guldberg, University of Queensland (B; Acropora millepora, Keppel Island, 2006).

While hyperoxic conditions in symbionts and host tissue will develop under natural diurnal light regimes (Dykens \& Shick, 1982, Shashar et al., 1993, Kühl et al., 1995), the long coevolution of both partners has adapted symbiotic hosts to tolerate hyperoxic conditions in terms of a stronger antioxidant defence system compared to non-symbiotic hosts (Dykens \& Shick, 1982, Richier et al., 2005). Furthermore, it has been shown that marker levels of oxidative lipid damage under natural light-induced tissue hyperoxia do not change significantly, indicating that symbiotic hosts may be able to cope with tissue hyperoxia (Richier et al., 2005). Early studies indicated that excessive light and temperature cause physiological stress in the symbiont, manifested as declines in the maximum quantum yield of photosystem II (PSII), photosynthetic rate, growth rate, and activity of ribulose 1,5-bisphosphate carboxylase/oxygenase (Rubisco) (Lesser \& Shick, 1989a, Lesser et al., 1990, Iglesias-Prieto et al., 1992, Lesser, 1996). At the same time, antioxidant enzyme activity was seen to increase, while the addition of exogenous antioxidants improved photosynthetic performance (Lesser, 1996). The consistent observation of increased antioxidative defence in both the symbiont and host under light and/or temperature stress (Shick et al., 1995, Downs et al., 2000, Yakovleva et al., 2004, Lesser, 2006, Flores-Ramírez \& Liñán-Cabello, 2007), in combination with the detection of reactive oxygen species (ROS; Dykens et al., 1992, Lesser, 1996, Tchernov et al., 2004, Suggett 
et al., 2008), has established the hypothesis that ROS play a significant role in coral bleaching. However, despite the evidence for the involvement of ROS in bleaching, the specific cause and effect relationship between inhibition of photosynthesis (light and dark reactions) and ROS generation in the symbiont, and the impacts on host physiology and symbiosis stability, have been the subject of debate for the last twenty years (Lesser et al., 1990, Lesser, 1997, Downs et al., 2002, Lesser, 2006, Venn et al., 2008, Baird et al., 2009, Fitt et al., 2009, Yakovleva et al., 2009).

Much of the focus has been on the symbiont as the more "environmentally sensitive partner" (Edmunds \& Gates, 2003). One mechanistic model of coral bleaching, which links molecular damage in the symbiont to the dissociation of the symbiosis, based on the generation of ROS, is called the "Oxidative Theory of Coral Bleaching" (as coined by Downs et al. 2002, based on the ideas and works of Malcolm Shick, Jim Dykens, lan Sandeman and Michael Lesser in the 1980's and 90's).

\section{Oxidative stress}

Oxidative stress describes a "disturbance in the prooxidant-antioxidant balance in favour of the former, leading to potential damage" (Sies, 1991). It has been identified as a key factor that contributes to cellular senescence and ageing in many organisms (Finkel \& Holbrook, 2000). Since the evolution of aerobic life is based on oxygen production and consumption for acquiring energy, the potential toxic properties of oxygen derivatives make mechanisms for counteracting or neutralizing adverse effects in organisms essential. The early studies of Gerschman et al. (1954) indicated similarities between the adverse effects of oxygen and ionizing radiation, suggesting that free radicals of oxygen could be the reason for the observed damaging effects. Since then, an extensive amount of literature has been published on oxygen toxicity and its cellular mechanisms (e.g. reviewed in Haugaard, 1968, Elstner, 1982, Fridovich, 1998). By definition, radicals are compounds with unpaired electrons (Halliwell \& Gutteridge, 2007). However, intermediate reduction products of activated oxygen are usually also considered as ROS. ROS commonly comprise singlet oxygen $\left({ }^{1} \mathrm{O}_{2}\right)$, hydroxyl radicals $\left(\mathrm{OH}^{\bullet}\right)$, superoxide anion radicals $\left(\mathrm{O}_{2}^{\bullet-}\right)$, perhydroxyl radicals $\left(\mathrm{HO}_{2}{ }^{\bullet}\right)$ and 
hydrogen peroxide $\left(\mathrm{H}_{2} \mathrm{O}_{2}\right)$. The basic reactions and conversions between these species are rather complex and multifaceted (Halliwell \& Gutteridge, 2007, Winterbourn, 2008). In the following section, principles and sites of generation that lead to the formation of ROS will be outlined.

\section{Photosynthesis under stress - ROS formation in the chloroplast}

The mechanisms that activate oxygen can be grouped into electron-transfer and energy-transfer reactions (Cadenas, 1995). The transfer of one electron to molecular oxygen in biological electron transport chains, reactions of carboncentred radicals with oxygen, or binding of oxygen to transition metals with unpaired electron spins, lead to the formation of superoxide radicals $\left(\mathrm{O}_{2}{ }^{\circ-}\right)$. In chloroplasts, solar radiation excites chlorophyll molecules, arranged in lightharvesting complexes (LHC), to a singlet state. The subsequent decay is usually used to channel excitation energy to the reaction centres, where it is utilized for charge separation. Excessive energy can be dissipated as radiative decay (fluorescence/heat), by involvement of the xanthophyll cycle or by the formation of triplet chlorophyll (Buchanan et al., 2000). Triplet chlorophyll, however, can interact with ground-state triplet oxygen to produce singlet oxygen $\left({ }^{1} \mathrm{O}_{2}\right)$ (Halliwell, 1984, Foyer et al., 1994). Additionally, generation of superoxide anions as a "malfunction" of ferredoxin-NADP ${ }^{+}$reductase (FNR) has been observed (Misra \& Fridovich, 1971). Due to the similar redox potential of ferredoxin $\left(\mathrm{Fd}_{\mathrm{red} / \mathrm{ox}}\right.$ $=-0.43 \mathrm{~V})$ and $\mathrm{O}_{2} / \mathrm{O}_{2}{ }^{\bullet-}(-0.45 \mathrm{~V})$, univalent reduction of oxygen can occur as a side reaction if electrons cannot be transferred to $\mathrm{NADP}^{+}$at photosystem I (PSI). The formation of superoxide by illuminated thylakoids as a result of direct transfer of electrons to oxygen is known as the Mehler reaction (Mehler, 1951).

Damage to plant cells is caused by the reaction of various ROS species with macromolecules (proteins, lipids or DNA), modifying their structure and thereby impairing their function (reviewed in Møller et al., 2007). The oxidation of thiolgroups by hydrogen peroxide in enzymes of the Benson-Calvin-cycle such as $\mathrm{NADP}^{+}$-glyceraldehyde-3-phosphate dehydrogenase (Halliwell, 1984), fructose biphosphatase and seduheptulose biphosphatase (Buchanan et al., 2000), impairs carbon dioxide fixation. As a consequence, sink-limitation for ATP and NADPH, with continuing energy absorption by photosystem II (PSII) chlorophylls, affects photosynthetic performance and can lead to increased pigment bleaching 
as well as D1-protein damage and/or insufficient synthesis of this protein (Buchanan et al., 2000). If excitation energy is not sufficiently disposed of by other processes, e.g. non-photochemical quenching (NPQ), and D1-protein turnover is too low, photoinhibition can occur. The impact of temperature and irradiance stress on the dinoflagellate pigments peridinin, diadinoxanthin, dinoxanthin and diatoxanthin levels has been outlined by some authors, emphasizing the photoprotective role of these compounds as part of the xanthophyll cycle (e.g. Prézelin, 1987, Brown et al., 1999, Venn et al., 2006). Whereas singlet oxygen has a short lifetime in aqueous solutions, it persists longer in hydrophobic environments like the interior of the thylakoid membrane (Halliwell, 1984). Interactions with the conjugated dienes of highly concentrated polyunsaturated fatty acid side-chains produce cytotoxic lipid peroxides, which further decompose to aldehydes such as malondialdehyde (MDA) or 4hydroxynonenal (4-HNE) and volatile hydrocarbons (ethane, ethylene, pentane). Subsequent alteration of membrane properties (see Richter, 1987) and damage to enzymes by lipid peroxides can lead to partial or complete impairment of membrane functionality and its bound protein/enzyme complexes, with subsequent energetic uncoupling in the case of thylakoid or mitochondrial membranes (Halliwell \& Gutteridge, 2007). Although the thylakoid membrane, and phospholipid membranes in general, are not very permeable to charged superoxide anions (Takahashi \& Asada, 1983), any reduction in membrane integrity could potentially result in leakage of ROS to the cytosol.

The Oxidative Theory of Coral Bleaching identifies the photosynthetic symbiont as the major contributor of ROS, based on three potential mechanisms: (1) Dysfunction of PSIl with D1 degradation, pigment bleaching and insufficient repair (Warner et al., 1999, Takahashi et al., 2004, Hill \& Ralph, 2006, Hill \& Ralph, 2008, Takahashi et al., 2009); (2) alteration of membrane properties and potential energetic uncoupling (Tchernov et al., 2004); and (3) inhibition of the Benson-Calvin cycle (Lesser, 1996, Jones et al., 1998). All three mechanisms have been shown to be valid for a stress response of Symbiodinium cells in high energy environments (light, UV, temperature) and are considered to be the main cellular impact sites in the algal cell that lead to the formation of ROS, with subsequent oxidative damage (Figure 1.5). 


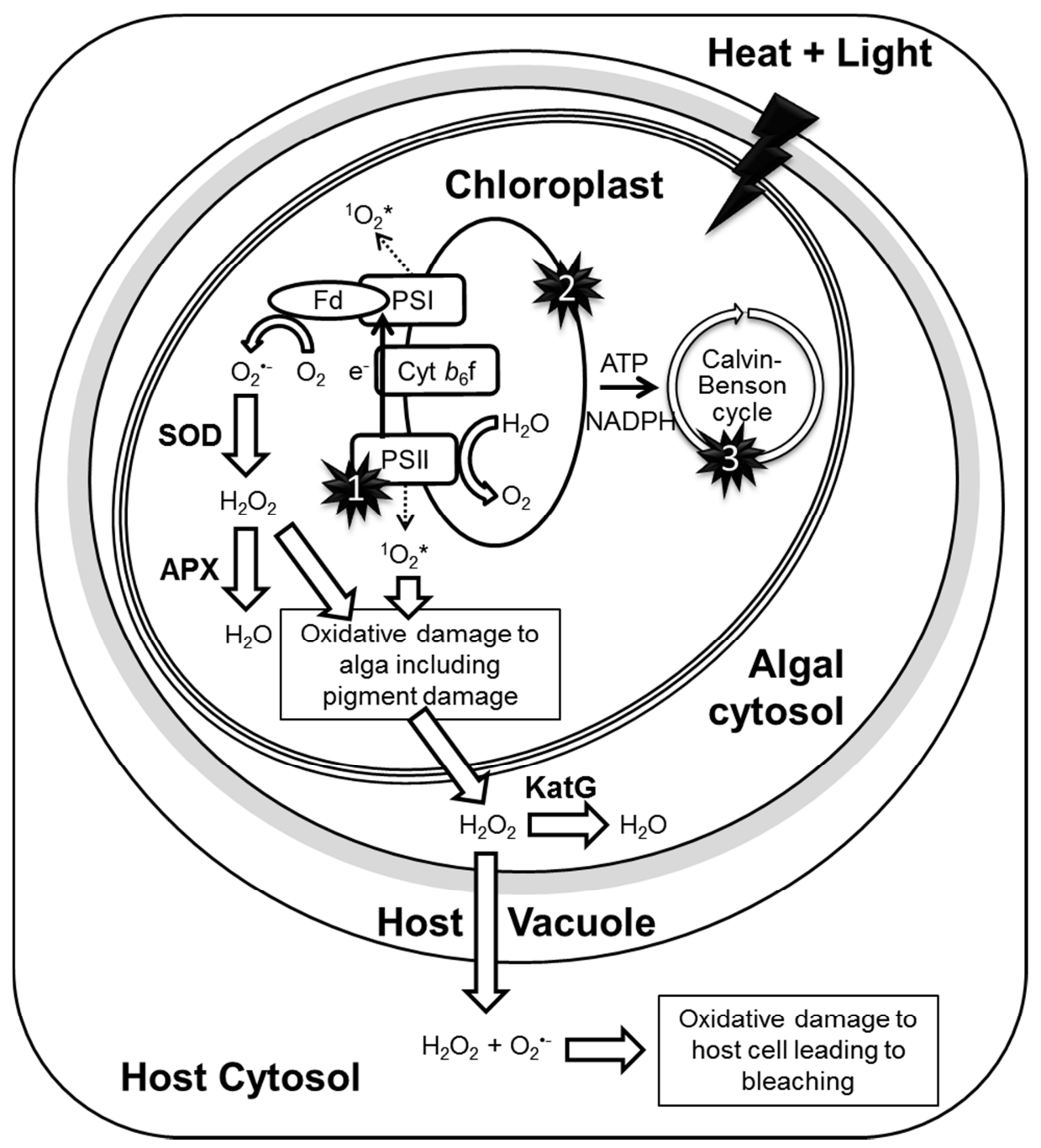

Figure 1.5. The Oxidative Theory of Coral Bleaching.

Shown are the primary impact sites in the symbiont under light and temperature stress (1-3) as outlined in the text. Generation of singlet oxygen $\left({ }^{1} \mathrm{O}_{2}{ }^{*}\right)$ and superoxide $\left(\mathrm{O}_{2}{ }^{\bullet}\right)$ at photosystems I and II (PSI and PSII) with inhibition of the dark reaction can overwhelm the antioxidative defence provided by superoxide dismutase (SOD) and ascorbate peroxidase (APX) activity in the chloroplast. Cytosolic hydrogen peroxide $\left(\mathrm{H}_{2} \mathrm{O}_{2}\right)$ is scavenged by catalase peroxidase (KatG). Accumulation of hydrogen peroxide leads to oxidative damage in the symbiont, and it eventually leaks into the host tissue leading to bleaching. Membranes (single black lines) and algal cell wall (grey) are indicated. Source: adapted from Venn et al. (2008).

As the production of ROS in chloroplasts, mitochondria, endoplasmic reticulum or peroxisomes is inevitable in organisms that produce or consume oxygen, a complex system to quench or reduce ROS fluxes has evolved, subsumed under the term 'antioxidative defence system'. Antioxidant defences are comprised of enzyme-mediated mechanisms, as well as direct scavenging of ROS by low molecular weight compounds (Halliwell \& Gutteridge, 2007). 


\section{Antioxidative enzyme mechanisms}

Enzymatic antioxidants such as superoxide dismutase (SOD; EC 1.15.1.1), ascorbate peroxidase (APX; EC 1.11.1.11), catalase (CAT; EC 1.11.1.6) and glutathione peroxidase (GPX; EC 1.11.1.9) detoxify superoxide to hydrogen peroxide and finally to water. Some of these enzymes are universally present, whereas others like APX are usually chloroplast-specific and therefore do not exist in animals. The concerted action of antioxidative enzyme mechanisms provides an effective scavenging mechanism of ROS. Spatial proximity of enzyme complexes to ROS-generating sites, as well as high enzymatic rate constants, allow for effective and rapid scavenging. Asada (2000) outlined microcompartmentalization of participating enzymes in higher plants, differentiating between thylakoidal and stromal scavenging systems for ROSremoval in the chloroplasts of higher plants (Figure 1.6).

The first line of defence represents the direct scavenging of superoxide by SOD and works via the disproportionation reaction:

$$
2 \mathrm{O}_{2}^{\bullet-}+2 \mathrm{H}^{+} \rightarrow \mathrm{H}_{2} \mathrm{O}_{2}+\mathrm{O}_{2}
$$

All three different SOD types, termed by their prosthetic metals as CuZnSOD, FeSOD and MnSOD (Kanematsu \& Asada, 1994), have been shown to be present in Symbiodinium cells (Lesser \& Shick, 1989a, Matta et al., 1992, Richier et al., 2003). In addition, various isoenzymes of SOD exist. Richier et al. (2003) identified at least seven different SOD activity bands in the sea anemone Anemonia viridis, while Alscher et al. (2002) mentioned 4-8 different isoforms in higher plants. In particular, isolated symbionts from $A$. viridis showed four MnSOD bands and two FeSOD isoenzymes (Richier et al., 2003), whereas cultured symbionts (ITS2 B1, M. Lesser, personal communication) showed three of the five SOD bands to be MnSOD (Lesser \& Shick, 1989a). Since the dismutation product of the SOD-reaction, hydrogen peroxide, inactivates CuZnSOD and FeSOD (see Kanematsu \& Asada, 1994 for general properties of CuZn-, Mn- and FeSODs), further reduction of hydrogen peroxide, e.g. by APX in chloroplasts, is essential (Asada, 1984). CuZnSOD occurs in the cytosol, mitochondria, chloroplasts and peroxisomes, and as extracellular CuZnSOD (EC- 
SOD), and represents the major form of SOD in plants and algae (Kanematsu \& Asada, 1994).

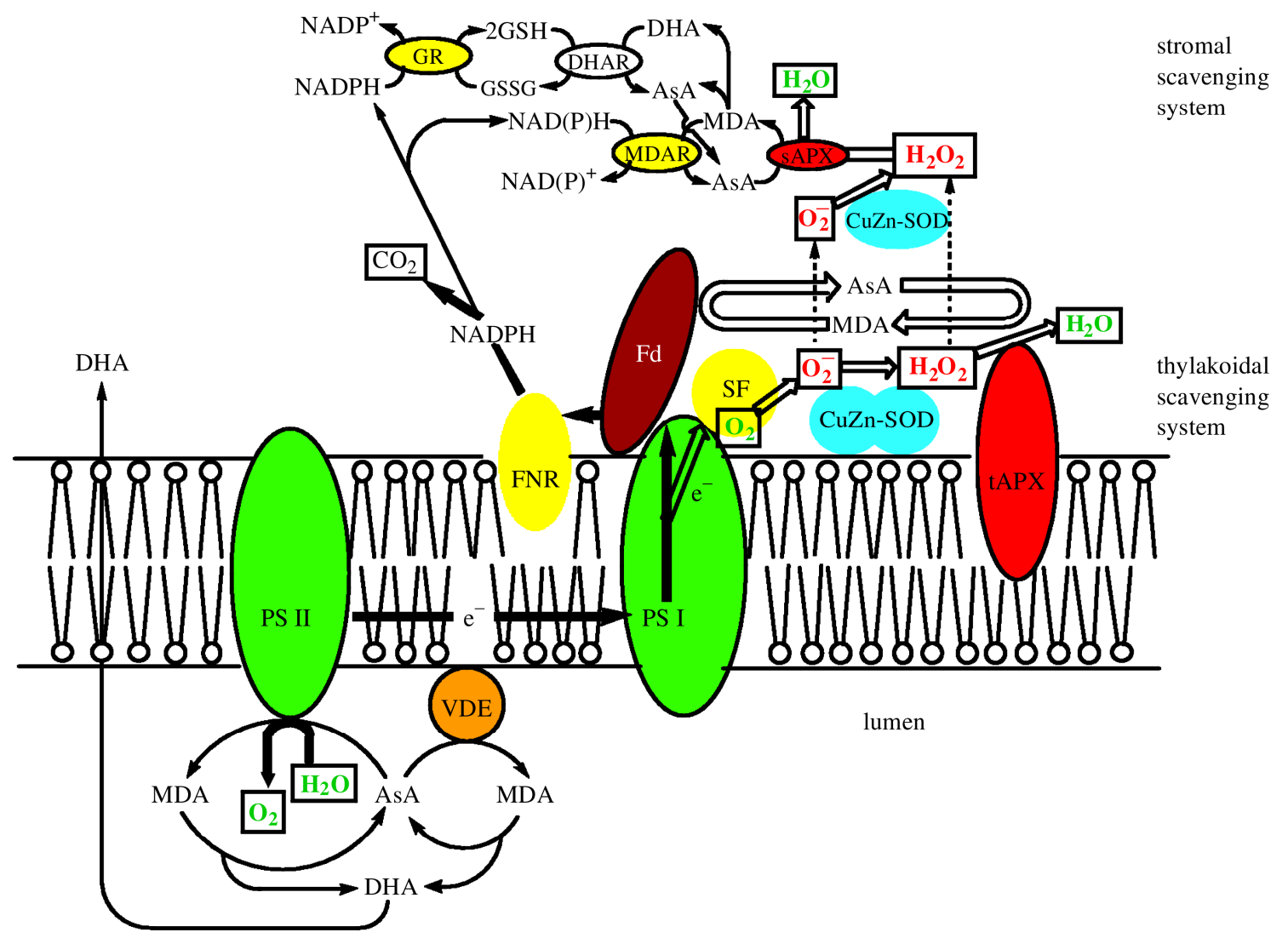

Figure 1.6. Antioxidant network in chloroplasts.

Schematic depiction of the network of antioxidative enzyme-mediated mechanisms of ROS removal associated with photosynthetic reaction centres in chloroplasts of higher plants, summarized by Asada (2000) as the 'water-water-cycle'. Shown is the splitting of water at PSII $\left(\mathrm{H}_{2} \mathrm{O} \rightarrow \mathrm{O}_{2}\right)$, production of superoxide as a result of photoreduction of $\mathrm{O}_{2}$ $\left(\mathrm{O}_{2} \rightarrow \mathrm{O}_{2}{ }^{\circ}\right)$, disproportionation of superoxide to $\mathrm{H}_{2} \mathrm{O}_{2}\left(\mathrm{O}_{2}{ }^{\circ-} \rightarrow \mathrm{H}_{2} \mathrm{O}_{2}\right)$ and reduction of hydrogen peroxide to water $\left(\mathrm{H}_{2} \mathrm{O}_{2} \rightarrow \mathrm{H}_{2} \mathrm{O}\right)$, as well as the associated pathways involving glutathione and ascorbate. APX, ascorbate peroxidase; AsA, ascorbate; CuZn-SOD, copper-zinc superoxide dismutase; DHA, dehydroascorbate; DHAR, dehydroascorbate reductase; Fd, ferredoxin; FR, Fd-NADP+ reductase; GR, glutathione reductase; MDA, monodehydroascorbate radical; MDAR, MDA reductase; SAPX, stromal APX; SF, stromal factor for enhanced photoreduction of dioxygen; tAPX, thylakoid-bound APX; VDE, violaxanthin de-epoxidase. Source: Asada (2000).

MnSOD occurs in the mitochondrial matrix and peroxisomes, but has also been identified as a thylakoid membrane-bound form in the chloroplasts of some plants (Kanematsu \& Asada, 1994); chloroplasts also contain FeSOD. Scavenging of hydrogen peroxide resulting from SOD activity is achieved by peroxidase and catalase. The presence of a chloroplastic glutathione peroxidase has been 
identified in Chlamydomonas and Arabidopsis (Navrot et al., 2006, Dayer et al., 2008), while no stromal catalase has been found in chloroplasts so far. However, a heme catalase associated with PSII membranes appears to have a significant role in the scavenging of hydrogen peroxide in chloroplasts (Sheptovitsky \& Brudvig, 1998). For Symbiodinium, the activity of a cytosolic catalase (Shick et al., 1995), later identified as catalase peroxidase (Leggat et al., 2007) and glutathione peroxidase (Downs et al., 2005, Kramarsky-Winter et al., 2009), has been shown.

\section{Low molecular weight non-enzymatic antioxidants}

A number of small molecules act as direct scavengers or co-substrates within the antioxidative system (Figure 1.6). While glutathione and ascorbate are watersoluble, lipophilic antioxidants such as tocopherols, carotenoids and mycosporine-like amino acids (MAAs) are specifically located in membranes to alleviate oxidative damage at these sites (Halliwell \& Gutteridge, 2007). In particular, a-tocopherol and carotenoids have been found to reduce the risk of lipid peroxidation by singlet oxygen (Fukuzawa, 2000, Munné-Bosch, 2005). High levels of MAAs and fluorescent host pigments, like the green fluorescent protein (GFP), have been suggested to shield Symbiodinium cells from UV- and high light-induced oxidative damage (e.g. Dunlap \& Yamamoto, 1995, Bou-Abdallah et al., 2006). Studies have also reported antioxidant activity for ubiquinone (e.g. Frei et al., 1990) and some compatible solutes like mannitol and dimethylsulfide (DMS) in Symbiodinium sp. and other marine algae (Shen et al., 1997, Jennings et al., 1998, Sunda et al., 2002, Miller, 2009, Yost \& Mitchelmore, 2009).

The tripeptide glutathione (Glu-Cys-Gly) plays an important role in this network, since it is a co-substrate for a number of pathways that remove products of oxidative damage. Additionally, it has been shown that glutathione acts in intercellular communication, drug detoxification, and radioprotection, and that it supports protein folding and degradation (reviewed in Sies, 1999, Franco et al., 2007, Halliwell \& Gutteridge, 2007). Its involvement in a number of antioxidative mechanisms makes glutathione an essential part of the cellular antioxidative defence system. The redox-state of the glutathione pool, i.e. the relative amounts of oxidized (GSSG) and reduced glutathione (GSH), has been suggested as one indicator of oxidative stress in a number of publications (reviewed in Sies, 1999, 
Lesser, 2006, Halliwell \& Gutteridge, 2007). Glutathione can also scavenge cellular hydrogen peroxide and organic peroxides $(\mathrm{ROOH}$, e.g., non-esterified fatty acid peroxides) by acting as a co-substrate for glutathione peroxidase via the reactions:

$$
\begin{array}{lll}
\mathrm{H}_{2} \mathrm{O}_{2}+2 \mathrm{GSH} & \rightarrow & \mathrm{GSSG}+2 \mathrm{H}_{2} \mathrm{O} \\
\mathrm{ROOH}+2 \mathrm{GSH} & \rightarrow & \mathrm{GSSG}+\mathrm{ROH}+\mathrm{H}_{2} \mathrm{O}
\end{array}
$$

Alternatively, it regenerates oxidized ascorbate in the chloroplast stroma via the Foyer-Halliwell-Asada cycle (Figure 1.7). By this means it prevents, in a concerted action with other pathways, potential damage by ROS and represents an essential component of the system (Halliwell, 1987). The Foyer-HalliwellAsada cycle is one of the central pathways in the antioxidant network and is not only linked to ascorbate peroxidase activity but also to regeneration of $\alpha$ tocopherol (Munné-Bosch, 2005), an important antioxidant in photosynthetic membranes for the limitation of lipid peroxidation.

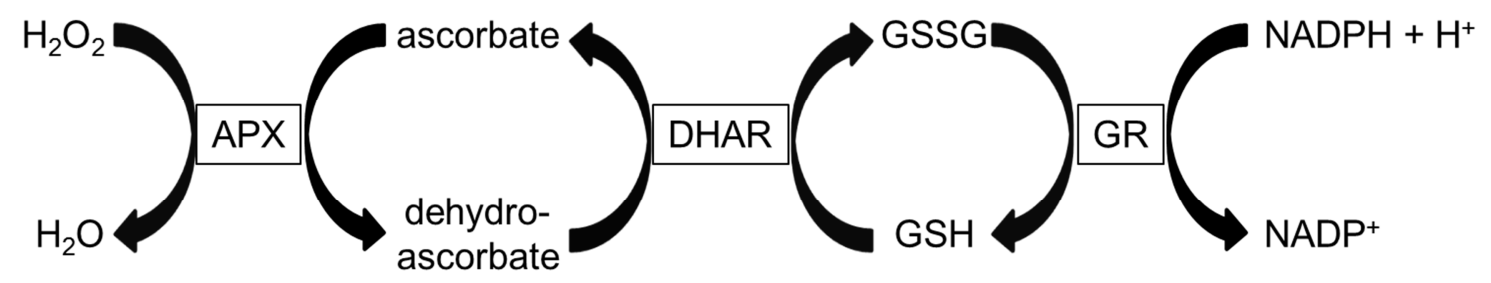

Figure 1.7. Foyer-Halliwell-Asada cycle.

Schematic depiction of the components of the ascorbate-glutathione cycle or FoyerHalliwell-Asada cycle in chloroplasts (compare Figure 1.6). Enzymes involved are ascorbate peroxidase (APX), dehydroascorbate reductase (DHAR) and glutathione reductase (GR). Glutathione as oxidized (GSSG) and reduced form (GSH). Redrawn from Halliwell (1987).

\section{The role of ROS in symbiosis breakdown}

While the involvement of ROS in the bleaching processes is supported by numerous studies, the downstream processes that lead to the dissociation of the symbiosis are not yet fully understood. As mentioned earlier, the bleaching definition is rather general. Fitt et al. (2001) differentiated between two types of stress bleaching: algal and animal stress bleaching. Algal bleaching is most likely based on the aforementioned effects of photoinhibition and pigment bleaching. 
However, some studies have illustrated processes of host cell death and detachment under thermal stress, with released symbionts exhibiting photosynthetic competence sometimes even at bleaching temperatures of $33^{\circ} \mathrm{C}$ (Gates et al., 1992, Ralph et al., 2001, Dunn et al., 2002, Dunn et al., 2004) or bleaching induction independent of photophysiological stress (Paxton et al., 2013, Tolleter et al., 2013). Therefore, bleaching might result not just from a dysfunction of the symbiont but from a dysfunction of the interaction between both partners. However the question of what exact process triggers the dissociation of the symbiosis remains open. The Oxidative Theory of Bleaching suggests a leakage of ROS from the symbiont to the host that affects the redox homeostasis of the host tissue. Support for this comes from a number of studies that have shown the upregulation of antioxidative enzymes as well as heat shock proteins (HSPs, molecular chaperones that stabilize proteins), in host tissues under thermal stress (Black et al., 1995, Fang et al., 1997, Sharp et al., 1997, Downs et al., 2000, Downs et al., 2002, Yakovleva et al., 2004, Fitt et al., 2009). ROS leakage to the host in non-necrotic Symbiodinium cells is based on the permeability of intact (or lipid peroxide compromised) membranes, and transmembrane transport of a particular ROS depends on its half-life, reactivity with macromolecules and diffusion coefficient (Halliwell \& Gutteridge, 2007, Winterbourn, 2008). Based on these properties, hydrogen peroxide (a very stable ROS) was suggested as the most likely candidate for leakage to the host (Lesser et al., 1990). The studies of Sandeman (2006) and Suggett et al. (2008) have shown that temperature- and light-challenged Symbiodinium cells release hydrogen peroxide in vitro. However, it has to be acknowledged that hydrogen peroxide also acts as a signalling compound, so cells need to regulate its compartmental concentrations. Thus, membranes are not always freely permeable and transmembrane diffusion is, to some degree, regulated by membrane lipid composition or the presence of facilitating channel proteins such as aquaporins (reviewed in Bienert et al., 2006).

Oxidative stress is a symptom of a disturbed cellular homeostasis. As such, the presence of reactive oxygen (and nitrogen) species (ROS/RNS) has led cells to respond not only by means of direct scavenging but also by altering their transcriptional profiles. In yeast and E. coli, transcription factors such as OxyR 
and Yap1 are redox-sensitive. In fact the specific oxidation pattern of cysteinyl residues acts as a sensor of a redox imbalance, with different ROS species causing different modulation patterns (Hancock, 2008). Thus ROS species can induce different genes while acting through the same transcription factor (D'Autréaux \& Toledano, 2007, Tachibana et al., 2009, Ouyang et al., 2011). In plants, nitric oxide ( $\mathrm{NO}^{\circ}$ ) has also been recognised as an important molecule involved in signal transduction. Nitric oxide is thought to be mainly produced by the activity of the enzyme nitric oxide synthase (NOS), but can emerge as a byproduct of nitric reductase (NR) under low oxygen tension and high nitrite levels (Rockel et al., 2002). Interaction of ROS and $\mathrm{NO}^{\circ}$ can give rise to a number of RNS. The reaction of superoxide and nitric oxide forms, for example, peroxynitrite (ONOO-), a highly reactive RNS (Halliwell \& Gutteridge, 2007). It is generally believed that the formation of peroxynitrite can significantly contribute to oxidative stress and downstream responses such as apoptosis in plants (ArasimowiczJelonek \& Floryszak-Wieczorek, 2011). Production of nitric oxide and peroxynitrite in Symbiodinium cells has been documented (Bouchard \& Yamasaki, 2008, Hawkins \& Davy, 2012, Hawkins \& Davy, 2013), while the presence of NOS and nitric oxide has been confirmed in different hosts (Colasanti et al., 2010, Safavi-Hemami et al., 2010).

\section{Processes of cell death}

Studies on the ultrastructural integrity of the symbiotic partners have shown that apoptosis and necrosis, as ultimate death responses, can occur in both partners under thermal stress (Dunn et al., 2002, Dunn et al., 2004). In the context of the Oxidative Theory of Bleaching, the redox imbalance caused or enhanced by additional ROS from the symbiont could initiate downstream regulatory pathways that cause necrosis and programmed cell death (PCD) in the host. Cnidarians, although basic metazoans, have a complex apoptosis network that is comparable to the one of higher vertebrates (Dunn et al., 2006, Zmasek et al., 2007, Lasi et al., 2010). Processes of cell death, be they related to necrosis, apoptosis or autophagy (both forms of PCD), are complex and partially overlap on a molecular level. For the cnidarian-dinoflagellate symbiosis, the discovery of conserved homologous protein sequences of a B-cell lymphoma 2 (Bcl-2) and a caspase (a 
family of cysteine proteases), provides clues for understanding the molecular processes of cell death in the host (Dunn et al., 2006, Richier et al., 2006). The initial apoptotic cascade can either follow an intrinsic (mitochondrial-mediated) or extrinsic (death-receptor-mediated) pathway. So far, available data mostly support the mitochondrial-mediated pathway for cnidarians. Bcl-2 family proteins are usually associated with an intrinsic pathway and include pro-apoptotic members such as Bak and Bax, as well as anti-apoptotic proteins such as Bcl-2 and Bcl- $\mathrm{x}_{\mathrm{L}}$ (Youle \& Strasser, 2008). The balance between proapoptotic BH3-only proteins (e.g., Bcl-2-interacting mediator of cell death [BIM], p53 upregulated modulator of apoptosis [PUMA], BH3 interacting-domain death agonist [BID]) and antiapoptotic Bcl-2 protein family members, affected by external stimuli such as free radicals, controls the mitochondrial apoptotic pathway. While integrity of the outer mitochondrial membrane is weakened by Bak and Bax through the formation of permeabilization pores, $\mathrm{Bcl}-2$ and $\mathrm{Bcl}-\mathrm{X}_{\mathrm{L}}$ counteract this process. The formation of mitochondrial membrane permeability transition pores (MPTP) can also be caused by a variety of secondary messengers of stress such as calcium, oxygen radicals, nitric oxide, ganglioside GD3, arachidonic acid and peroxynitrite, as well as fatty acids and their oxidation products such as hydroxynonenal (Vieira et al., 2000). Loss of mitochondrial integrity leads to the release of proapoptotic molecules such as cytochrome c, SMAC/DIABLO (second mitochondrial activator of caspases/direct inhibitor of apoptosis binding protein with low isoelectric point), and the serine protease HtrA2/Omi that promotes caspase activation. Activity of caspases, such as the executioner caspases $-3,-6$ and -7 , then leads to substrate cleavage and apoptosis (Elmore, 2007). Activity of these caspases is linked to mitochondrial integrity, because it can be initiated by the initiator caspase-9 or blocked by a group of IAP (inhibitor of apoptosis) proteins. The two factors are, however, dependent on the release of mitochondrial SMAC/DIABLO, which inactivates IAP proteins and the formation of a heptameric protein (aptosome), which activates caspase-9. Released cytochrome $\mathrm{c}$ will react with APAF1 (apoptotic protease-activating factor 1), and is therefore essential for the formation of aptosomes and the initiation of the apoptotic cascade (Elmore, 2007, Hotchkiss et al., 2009, Figure 1.8A). Structural homologues for components of the mitochondrial-mediated apoptosis pathway have been found for the sea anemones Aiptasia pallida (Dunn et al., 2006), 
Anemonia viridis (Richier et al., 2006) and Nematostella vectensis (Oberst et al., 2008) and the hydrozoan Hydra (Lasi et al., 2010, Figure 1.8B). RNA interference (RNAi) assays targeting a homologue of the caspase- 3 have been shown to prevent caspase-mediated apoptosis in A. pallida (Dunn et al., 2007a).
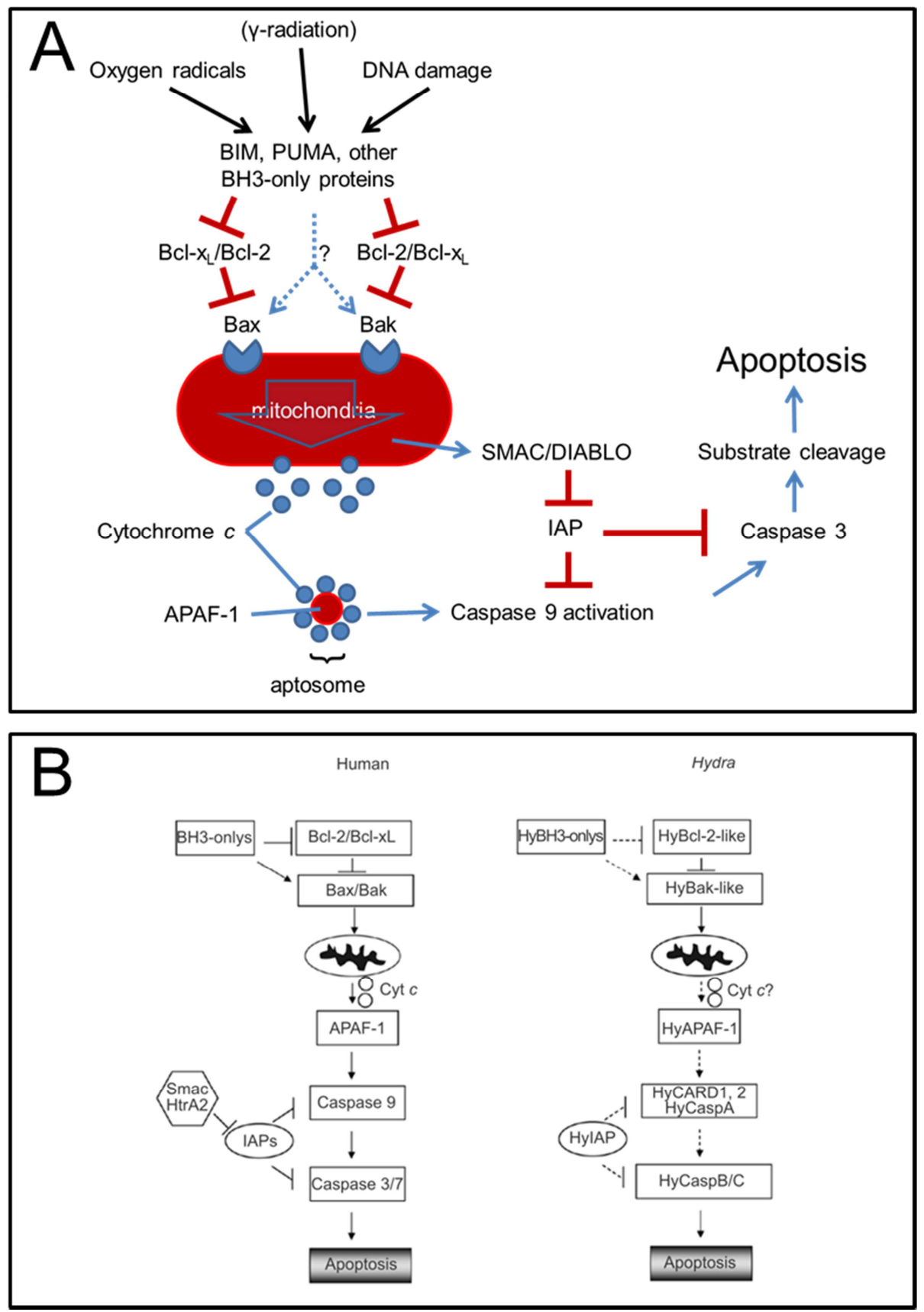

Figure 1.8. Mitochondrial apoptosis pathway.

(A) Schematic depiction of the intrinsic apoptotic cascade in humans and (B) the comparison with homologues from the network of Hydra. Homologues of these components have also been found in a number of cnidarians (see text). Dashed lines indicate the possibility that proapoptotic $\mathrm{BH} 3$ molecules might act directly on Bax and Bak to induce mitochondrial permeability. Figures adapted from Hotchkiss et al. (2009) (A) and Lasi et al. (2010) (B). 


\section{A potential role of calcium in the apoptotic cascade}

Release of intact symbionts from corals and anemones under high and low temperature stress has drawn attention to the involvement of calcium in the mechanisms of exocytosis and host cell detachment. While Steen \& Muscatine (1987) argued that low temperature might have stimulated calcium-related exocytosis in the anemone Aiptasia pulchella, Gates et al. (1992) suggested that temperature-induced membrane thermotropism permits passive influx of ions such as calcium. Increased calcium levels would then compromise cellular adhesion of host cells and affect the assembly of cytoskeletal filaments, causing the observed host cell detachment. Whereas the study of Sawyer \& Muscatine (2001) was unable to confirm the idea of thermal thermotropism and changes in intracellular calcium, other studies have demonstrated an increase in the intracellular calcium concentration with heat exposure in the absence of an external calcium supply (Huang et al., 1998, Sandeman, 2006). Chelation and depletion of intracellular calcium stores on the other hand suppressed the bleaching effect (Fang et al., 1997, Huang et al., 1998). Similar to conflicting results about the involvement of calcium in cnidarian bleaching, evidence for the involvement of calcium in apoptosis in mammalian cells is controversial, though it is generally believed that calcium and its major store, the endoplasmic reticulum (ER), play an important role in apoptotic events (Guo et al., 2009). In mammals, a pathway that directly links disturbance in calcium homeostasis to caspase activity exists in the form of the ER apoptotic pathway. In this pathway, calpain, a calcium-dependent cystein protease in the ER, activates cytosolic caspase 12, which in turn activates caspase 9 and subsequently caspase 3 (Ferri \& Kroemer, 2001). Furthermore, there is an indication that a calpain similar to caspase 1 and 3 can cleave $\mathrm{Bcl}-\mathrm{X}_{\mathrm{L}}$, converting it from an anti-apoptotic to a pro-apoptotic state (Nakagawa \& Yuan, 2000). Excessive cytosolic calcium can trigger a mitochondrial calcium overload, resulting in mitochondrial dysfunction by formation of MPTPs with subsequent apoptotic downstream effects as described earlier (Ferri \& Kroemer 2001).

Little is known about the regulation of apoptotic pathways and the particular role of calcium in the breakdown of the coral symbiosis. However, advancement in the general understanding of apoptotic networks and the introduction of molecular 
tools such as RNAi and genome sequencing to questions of coral health will promote future research in this area.

\section{Partner flexibility as an adaptive mechanism?}

As a result of the mosaic nature of the coral holobiont, it was suggested that perhaps "specificity changes as environmental conditions do" and that "although bleaching may represent instability in the short term, it promotes long-term stability by enhancing survival chances [...] under conditions that are not those of the pre-stress environment" (Buddemeier \& Fautin, 1993). Thus, bleaching was proposed as an adaptive mechanism; adaptive in a sense that it alters the holobiont's composition to enhance its fitness under the conditions of its environment (Adaptive Bleaching Hypothesis; Buddemeier et al., 2004). Changes in composition of the symbiont population can occur as result of: (1) frequency shifts in the symbiont types within the host ("shuffling"); and (2) expulsion and acquisition of novel types from the environment ("switching") (Baker, 2003). While the potential for symbiont shifts in some corals is evident (e.g., Toller et al., 2001, Lewis \& Coffroth, 2004, Chen et al., 2005a, Berkelmans \& van Oppen, 2006, Jones, 2008), the presence of cryptic types, i.e., types that are present at a very low density, make distinguishing between shuffling and true switching with the emergence of novel symbiont types, difficult. Moreover, differentiation between both mechanisms in field studies is complicated further by potential sampling bias due to differential colony mortality (Baker, 2004). On the other hand, there is evidence that some associations are highly specific and do not change throughout bleaching events, as is the case for the vertical transmitting PoritesSymbiodinium C15 association (LaJeunesse et al., 2003, Baker, 2004, Baker et al., 2008, Stat et al., 2008a, Stat et al., 2009). To what degree flexibility and specificity in host-Symbiodinium pairings can be related to thermal sensitivity is still under debate (Putnam et al., 2012b). 


\section{Scope of this thesis}

The overall aim of this study was to investigate the link between Symbiodinium diversity and thermal sensitivity. In particular, the role of key antioxidants in explaining differential bleaching susceptibility between symbiont types in vitro, as well as between different coral holobionts, was studied. Furthermore, variation of major enzymatic antioxidants on the amino acid level was investigated in a number of Symbiodinium types. This thesis pursued the following objectives:

1) Monitor major antioxidant pathways under sublethal and lethal thermal stress to determine to what degree the level of activity differs between Symbiodinium types in culture, and how their responses are connected to cell viability in terms of photosynthesis and growth.

Hypotheses: (a) Antioxidant defences in Symbiodinium will increase in response to elevated temperature and declining photosynthetic performance; (b) Some parts of the antioxidant network will differ in their response between types at given temperatures; (c) Overall viability of types will be related to $(b)$.

2) Integrate multiple antioxidants into a network response, to identify activity shifts in thermally- sensitive and -tolerant Symbiodinium types in culture

Hypothesis: (a) Closely connected elements of the antioxidant network will be correlated in their response to temperature; (b) The integrated network response will highlight the most responsive parts (i.e. main shifts) in the network at given temperatures.

3) Develop molecular tools to measure the transcriptomic responses of major enzymatic antioxidants in Symbiodinium, and contrast transcriptomic and proteomic antioxidant responses under thermal stress.

Hypothesis: (a) Antioxidant defences in Symbiodinium will increase in response to elevated temperature and declining photosynthetic performance; (b) If enzymatic antioxidants are transcriptionally regulated, their gene expression will precede increases in enzymatic activity. 
4) Investigate the degree of sequence divergence and phylogenetic relatedness of enzymatic antioxidants on the clade and type level in Symbiodinium.

Hypothesis: (a) The genetic radiation of the genus will be reflected in mutations to antioxidant genes; (b) Antioxidant gene mutations will relate to variations in amino acid sequences of enzymatic antioxidants; (c) Sequence similarities of enzymatic antioxidants from different types will be higher between types of the same clade.

5) Compare the activity of antioxidants in both partners under thermal stress between corals with differing thermal sensitivities under field conditions.

Hypothesis: (a) Antioxidant defences in Symbiodinium will increase in response to elevated temperature and declining photosynthetic performance; (b) Antioxidant defences of the coral host will increase in response to (a); (c) Coral bleaching at high temperatures will occur as a result of (a) and (b).

As this thesis investigates a number of different aspects that are relevant for linking symbiont diversity to antioxidant defences under thermal stress, each chapter is written as a manuscript in preparation for submission. Thus, there may be some overlap in content between chapters, particularly in the introductions of each chapter. 


\section{Antioxidant plasticity and thermal sensitivity in the coral endosymbiont Symbiodinium sp.}

\section{Introduction}

Worldwide, shallow tropical coral reef systems experience severe ecological impoverishment as a result of local stressors and the effects of global climate change (Pandolfi et al., 2003). The success of these ecosystems lies in the endosymbiosis between cnidarians (Class: Anthozoa) and dinoflagellates from the genus Symbiodinium. The Symbiodinium cells supply photosynthetic carbon to the host and enable fast calcification rates, while benefiting from the provision of inorganic nutrients such as ammonium and phosphate (Muscatine \& Porter, 1977). The resulting advantages, including the recycling of critical nutrients, partially explains the success of coral reefs in the oligotrophic waters of tropical coastal areas. Localized breakdown of this obligate symbiosis (i.e. coral bleaching), as a result of reduced salinity, aerial exposure, high irradiance or disease, occurs on all reefs and contributes to the natural turnover of corals (Sheppard et al., 2009). However, mass bleaching and mortality events that have large scale impacts on multiple species are typically driven by increasing sea surface temperatures (SST) (Jokiel \& Coles, 1990, Glynn, 1993). Such mass bleaching events, alongside ocean acidification, are considered a major threat to the survival of coral reefs (Hoegh-Guldberg et al., 2007, Carpenter et al., 2008).

The dinoflagellate genus Symbiodinium exhibits a great deal of genetic diversity (at least nine clades and hundreds of subclades based on their ITS2 phylogeny) and associates with at least five animal phyla (Cnidaria, Mollusca, Foraminifera, Protozoa, Porifera) (Baker, 2003, LaJeunesse, 2005, Pochon \& Gates, 2010). As conspecific Symbiodinium types can associate with different hosts, the bleaching sensitivity of corals to environmental stress is determined by the characteristics of both animal host and symbiont (Loya et al., 2001, Stimson et al., 2002, Abrego et al., 2008, Baird et al., 2009). Oxygen concentrations within photosynthetic symbioses vary greatly over the diel cycle and can also exacerbate these forms of stress. By day, photosynthesis by endosymbionts leads to hyperoxic host tissues; by night respiration by endosymbionts and host tissue leads to a state of 
hypoxia, especially in the deep tissues of the calicoblastic body wall (Dykens \& Shick, 1982, Shashar et al., 1993, Kühl et al., 1995). Oxygen supersaturation $(>200 \%)$ by day and rapid changes in host tissue oxygenation can promote the generation of reactive oxygen species (ROS; Halliwell \& Gutteridge, 2007). In response, coral hosts upregulate their antioxidant defences, compared to the non-symbiotic state, to the extent that significant lipid and protein oxidation is avoided (Dykens \& Shick, 1982, Richier et al., 2005).

Excessive light and temperature have been shown to cause physiological stress in Symbiodinium in the form of declines in growth and photosynthetic rate, maximum quantum yield of photosystem II (PSII) and activity of ribulose 1,5bisphosphate carboxylase/oxygenase (Rubisco) (Iglesias-Prieto et al., 1992, Lesser, 1996, Jones et al., 1998). At the same time, antioxidant enzyme activity has been shown to increase, and the addition of exogenous antioxidants partially improves photosynthetic parameters such as maximum quantum yield of PSII $\left(F_{v} / F_{m}\right)$ and Rubisco activity (Lesser, 1996, Lesser, 1997). Concurrent observation of increased antioxidative defences in both partners (Shick et al., 1995, Downs et al., 2000, Yakovleva et al., 2004, Flores-Ramírez \& LiñánCabello, 2007) and detection of ROS in cultured Symbiodinium under light and/or temperature stress (Tchernov et al., 2004, Suggett et al., 2008, McGinty et al., 2012), has established the hypothesis that ROS play a significant role in the onset of coral bleaching. Under environmental stress, a profound redox imbalance in the symbiont might lead to the leakage of ROS to the host tissue (Sandeman, 2006, Suggett et al., 2008), challenging and potentially overwhelming the antioxidative capacity of the host. Furthermore, since ROS can act as direct or indirect (via the oxidation of amino acid residues in functional proteins) signalling molecules, leakage of ROS from the symbionts will affect cellular processes in the host cell (Mullineaux et al., 2006). This 'Oxidative Theory of Coral Bleaching' assumes a unidirectional flow of excessive prooxidants from symbiont to host and highlights the potential role that the dinoflagellate symbiont's antioxidative capacity plays in the regulation of coral health (Lesser, 1997, Downs et al., 2002).

Oxidative stress is defined as the "disturbance in the prooxidant-antioxidant balance in favour of the former, leading to potential damage" (Sies, 1991). It has 
been identified as one key factor in explaining fundamental processes of cellular senescence and ageing in organisms (Finkel \& Holbrook, 2000). A number of oxygen intermediates are generated in the pathways of aerobic metabolism or photosynthesis, and can also occur as products of non-enzymatic autoxidation of macromolecules (Foyer et al., 1994, Halliwell \& Gutteridge, 2007, Navrot et al., 2007). A complex antioxidant network, comprised of enzymatic and nonenzymatic pathways, maintains the redox balance and serves as a crucial element of cellular homeostasis (Apel \& Hirt, 2004). Here, the term 'antioxidant network' is used in a narrower sense to refer to two interconnected systems: the glutathione system and the pathway from superoxide dismutase (SOD) to ascorbate peroxidase (APX), which is involved in scavenging two oxygen intermediates: superoxide $\left(\mathrm{O}_{2}{ }^{\circ}\right)$ and hydrogen peroxide $\left(\mathrm{H}_{2} \mathrm{O}_{2}\right)$ (Figure 2.1). Both systems are connected by the ascorbate-glutathione cycle (Foyer-Halliwell-Asada cycle), which utilizes reduced glutathione to regenerate ascorbate for APX activity in the chloroplast (Asada, 2006). The tripeptide glutathione in its reduced form (GSH) represents a major antioxidant in the cell, and the glutathione pool in tissues of higher animals and plants usually consists of more than $90 \% \mathrm{GSH}$ (Halliwell \& Gutteridge, 2007). In addition to its ability to directly scavenge a number of prooxidants, glutathione is also involved in the removal of xenobiotics and organic peroxides via the activity of glutathione S-transferase (GST), and it plays an important role in protein modification and cell signalling (Foyer \& Noctor, 2011). Glutathione therefore represents an essential component of the antioxidant network and operates in concert with other pathways to prevent damage by ROS. 


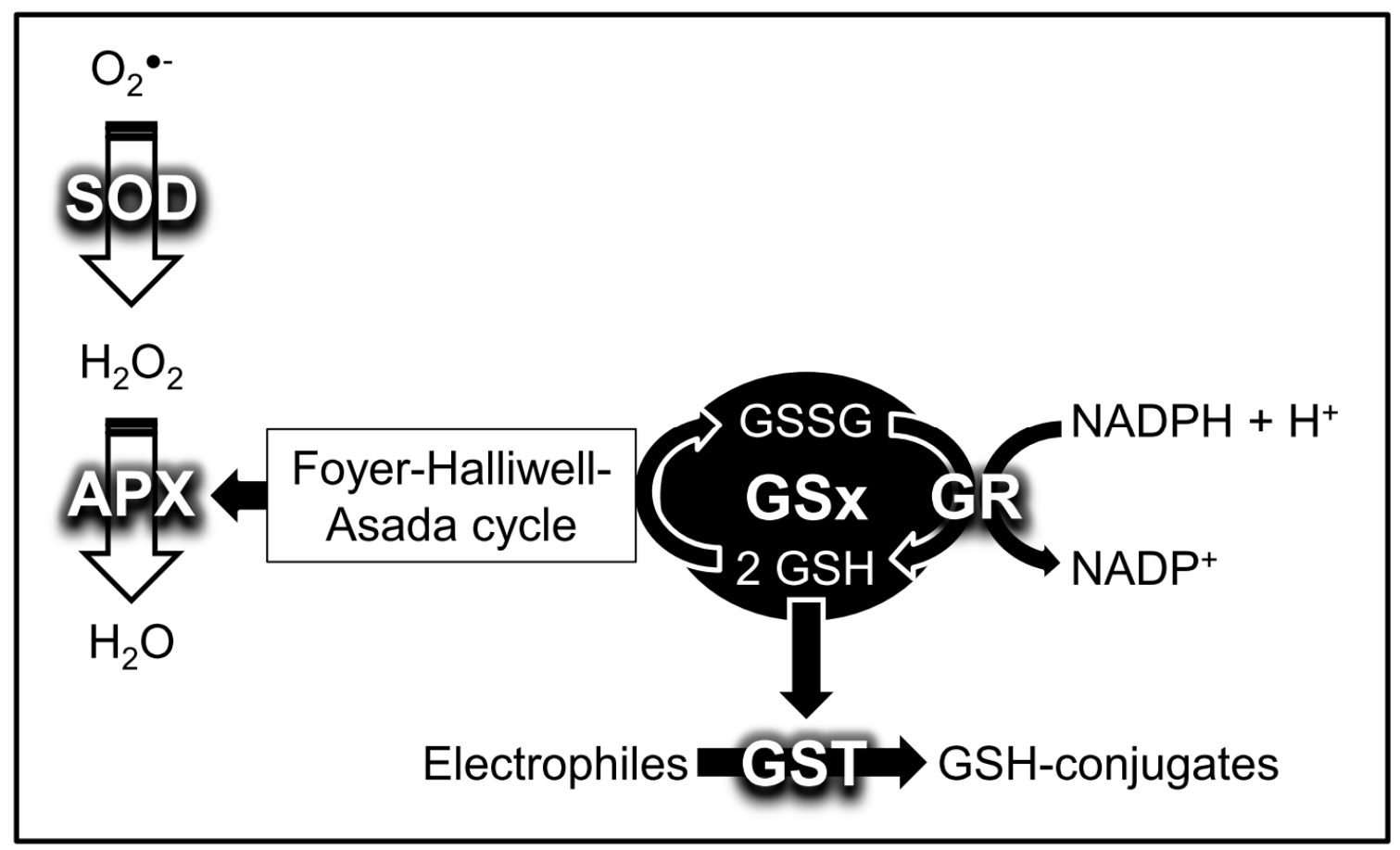

Figure 2.1. Components of the antioxidant network assessed in this study.

Connection of elements of the antioxidant network investigated in this study, disregarding subcellular localisation. Superoxide dismutase (SOD), ascorbate peroxidase (APX), total glutathione content (GSx), glutathione disulphide (GSSG), reduced glutathione $(\mathrm{GSH})$, glutathione reductase (GR), glutathione S-transferase (GST). Shown are enzymes of the superoxide removal pathway (white arrows) and pathways involving glutathione (black arrows).

The potential involvement of ROS and antioxidative defences in Symbiodinium stress physiology and coral bleaching has been widely acknowledged, however few studies have systematically characterised the link between antioxidative capacity and thermal resistance in different Symbiodinium genotypes. While it has been illustrated that cellular ROS levels differ between these types (McGinty et al., 2012), significant differences in hydrogen peroxide production under temperature stress in thermally tolerant and susceptible Symbiodinium genotypes have only been found at very high light levels (for cultures) of $1000 \mu \mathrm{mol}$ quanta $\mathrm{m}^{-2} \mathrm{~s}^{-1}$ (Suggett et al., 2008).

Here, I hypothesized that the differences in the thermal sensitivity of Symbiodinium genotypes are directly related to their capacity to withstand thermally induced oxidative stress. In order to investigate this hypothesis, the relationship between elevated temperature, cell viability and activity of the antioxidant network was examined in four cultured Symbiodinium genotypes. In 
particular, it was tested whether the activity of elements of the antioxidant network fundamentally differs between types, and how thermal stress affects enzymatic scavenging of ROS and the dynamics of the glutathione system. This study provides a mechanistic view of temperature-induced activity shifts in the antioxidant network of Symbiodinium genotypes with varying thermal susceptibilities.

\section{Material and Methods}

\section{Experimental setup}

Batch cultures of Symbiodinium B1 (culture ID Ap1), C1 (CCMP2466), E (CCMP421) and F1 (Mv) that had been continuously maintained at $25^{\circ} \mathrm{C}$ were exposed to temperatures of $25.1^{\circ} \mathrm{C} \pm 0.6^{\circ} \mathrm{C}, 29.0^{\circ} \mathrm{C} \pm 0.2^{\circ} \mathrm{C}$ and $33.3^{\circ} \mathrm{C} \pm 0.3^{\circ} \mathrm{C}$ over 14 days in temperature-controlled tanks (Figure 2.2). Cultures were grown in $2 \mathrm{~L}$ round-bottom flasks, containing silica-free sterile f/2-medium, $\mathrm{pH} 8.1$ (Guillard \& Ryther, 1962), based on synthetic seawater (salinity 34; Instant Ocean sea salt, Spectrum Brands Inc., USA). Cultures were aerated with filter-sterilized air (0.22 $\mu \mathrm{m})$ and grown on a constant 12-h/12-h light/dark cycle under an irradiance of 40-50 $\mu \mathrm{mol}$ quanta $\mathrm{m}^{-2} \mathrm{~s}^{-1}$ (cool white fluorescent tubes, Philips 36W/840). The experimental settings were chosen based on preliminary experiments to achieve several discrete stress levels and physiological responses. All four cultures were tested simultaneously, after being acclimated to the experimental settings for ten days and the experiment was replicated four times with fresh batch cultures. After sampling on Day 0 , the temperature in the heated tanks was increased from $25^{\circ} \mathrm{C}$ by $1.67 \pm 0.40^{\circ} \mathrm{C} \mathrm{h}^{-1}$ for the $29^{\circ} \mathrm{C}$ tank and $1.44 \pm 0.30^{\circ} \mathrm{C} \mathrm{h}^{-1}$ for the $33^{\circ} \mathrm{C}$ tank; these increases took place during the dark phase. Temperature was monitored via HOBO Pendant ${ }^{\circledR}$ data loggers (Onset Computer Corporation, Bourne, MA, USA).

Initial cell density was approximately $1 \times 10^{5}$ cells $\mathrm{mL}^{-1}$ and cultures were sampled by removing $50 \mathrm{~mL}$ aliquots ( $\mathrm{N}=9$ per culture) of culture medium containing Symbiodinium cells on Days $0,1,3,7$ and 14. Aliquots were pelleted (5 min, $2000 \times \mathrm{g}, 25^{\circ} \mathrm{C}$ ) and the pellet was immediately flash frozen and stored at $-80^{\circ} \mathrm{C}$. To ensure that the cultures were maintained in exponential growth (based on 
preliminary growth experiments), flasks were replenished with $500 \mathrm{~mL}$ of $\mathrm{f} / 2$ medium after Day 3 (for B1, E and F1) and Day 7 (all cultures).
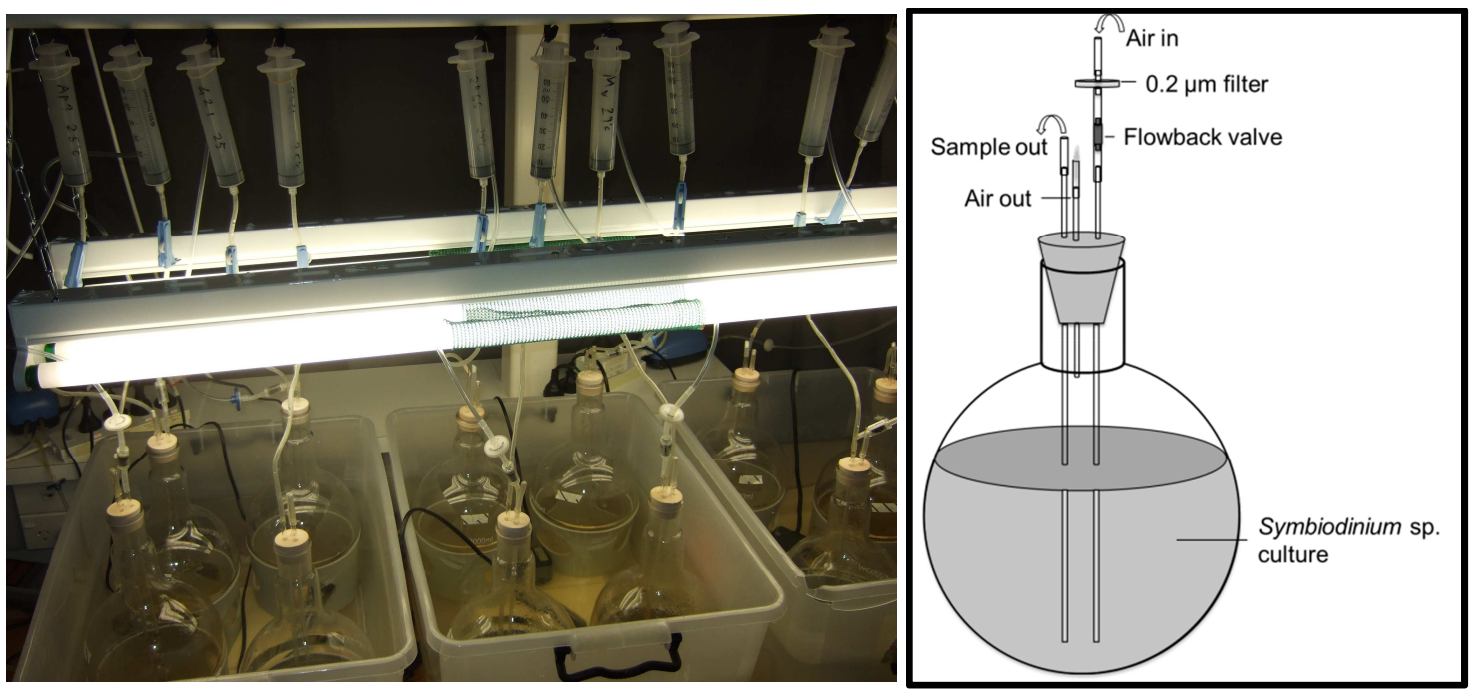

Figure 2.2. Experimental setup.

Experimental setup of four Symbiodinium cultures, exposed to three temperatures in multiple replicate runs (left). Cultures were maintained in sterile, aerated $2 \mathrm{~L}$ flasks (right).

\section{Cell viability}

Growth, maximum quantum yield of PSII $\left(\mathrm{F}_{\mathrm{v}} / \mathrm{F}_{\mathrm{m}}\right)$ and chlorophyll a $(\mathrm{Chl}$ a) content were monitored as proxies for cell viability in all cultures. $F_{v} / F_{m}$ was measured using an Imaging-PAM chlorophyll fluorometer (MI 7-10, SI 10, Gain 1-3, Damp 2-3, saturating width $0.8 \mathrm{~s}$; Heinz Walz $\mathrm{GmbH}$, Effeltrich, Germany) by pelleting 5$10 \mathrm{~mL}$ of each Symbiodinium culture (5 min, $2000 \times \mathrm{g}, 25^{\circ} \mathrm{C}$ ) and resuspending the pellet in $1 \mathrm{~mL} \mathrm{f} / 2$-medium. Three technical replicates (200 $\mu \mathrm{L}$ each) were measured after 20 min dark adaptation in a black flat-bottom microtiter plate. Cell density was assessed by haemocytometer counts $(\mathrm{N}=6)$ and the specific growth rate $(\mu)$ determined using the equation:

$$
\mu=\left(\ln N_{2}-\ln N_{1}\right) /\left(t_{2}-t_{1}\right)
$$

where $N_{1}$ and $N_{2}$ are densities at times $t_{1}$ and $t_{2}$ (Guillard, 1973). Chl $a$ of one of the frozen $50 \mathrm{~mL}$ pellets was extracted over $48 \mathrm{~h}$ in $1-2 \mathrm{~mL} \mathrm{~N}, \mathrm{~N}$ dimethylformamide at $4^{\circ} \mathrm{C}$ in the dark. For spectrophotometric measurements, extracts were centrifuged to remove cellular debris $(3 \mathrm{~min}, 3900 \times \mathrm{g})$ and absorbance of $3 \times 200 \mu \mathrm{L}$ (technical replicates) determined at $646.8 \mathrm{~nm}, 663.8$ $\mathrm{nm}$ and $750 \mathrm{~nm}$ in 96 well plates (UVStar, Greiner Bio-One $\mathrm{GmbH}$, 
Frickenhausen, Germany). Chl a concentrations $\left(\mu \mathrm{g} \mathrm{mL}^{-1}\right)$ were determined after optical path length correction $(0.555 \mathrm{~cm})$ using the equation:

$$
\text { Chl } \mathrm{a}=12.00 \times \mathrm{A}_{663.8 \mathrm{~nm}}-3.11 \times \mathrm{A}_{646.8 \mathrm{~nm}}
$$

where $A_{663.8 n m}$ and $A_{646.8 n m}$ represent the turbidity corrected $\left(A_{750 \mathrm{~nm}}\right)$ absorbances at 663.8 and $646.8 \mathrm{~nm}$ (Porra et al., 1989).

\section{Cell lysis}

For enzyme measurements, five frozen $50 \mathrm{~mL}$ pellets per time-point were pooled

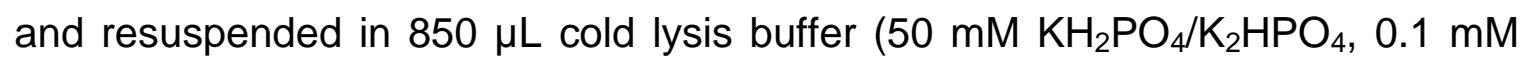
EDTA, 10\% [v/v] glycerol, protease inhibitor (P9599, Sigma-Aldrich), $\mathrm{pH}$ 7.0). After addition of $200 \mathrm{mg}$ glass beads (710-1180 $\mu \mathrm{m}$; Sigma-Aldrich), cells were lysed in a bead mill $\left(50 \mathrm{~Hz}, 3 \mathrm{~min}, 4^{\circ} \mathrm{C}\right.$; Qiagen tissue lyser, Qiagen N.V., Hilden, Germany). All lysates were centrifuged $\left(16000 \times \mathrm{g}, 5 \mathrm{~min}, 4^{\circ} \mathrm{C}\right)$ and supernatants aliquoted and frozen at $-80^{\circ} \mathrm{C}$ until further analysis. Total aqueous soluble protein content was determined using the improved Bradford assay with BSA as a protein standard (Zor \& Selinger, 1996).

For glutathione determination, samples were processed within seven days of each experimental run. Two frozen $50 \mathrm{~mL}$ pellets were washed, pooled in $350 \mu \mathrm{L}$ $5 \%[\mathrm{w} / \mathrm{v}]$ 5-sulfosalicylic acid and lysed as above. Lysates were centrifuged $\left(16000 \times \mathrm{g}, 5 \mathrm{~min}, 4^{\circ} \mathrm{C}\right.$ ) and $200 \mu \mathrm{L}$ of the protein-free supernatant used for measurement of total glutathione content (GSx) and glutathione disulphide content (GSSG). Remaining supernatant was discarded and the pellets frozen at $-80^{\circ} \mathrm{C}$. For protein determination, pellets were resuspended and incubated in 0.2 $M$ Tris on an orbital shaker $\left(10 \mathrm{~min}, 4^{\circ} \mathrm{C}\right)$. After centrifugation $(16000 \times \mathrm{g}, 5 \mathrm{~min}$, $4^{\circ} \mathrm{C}$ ), total aqueous soluble protein content was measured as before.

\section{Glutathione content}

GSx was assessed using the DTNB-recycling assay in a microtiter format (Baker et al., 1990). For GSx determination, $20 \mu \mathrm{L}$ of sample or glutathione standard (0500 pmol GSSG/20 $\mu \mathrm{L}$ ) were used in a $200 \mu \mathrm{L}$ reaction mixture containing potassium phosphate $(90 \mathrm{mM}, \mathrm{pH} 7.5)$, EDTA $(0.9 \mathrm{mM})$, glutathione reductase (1 $\mathrm{U} / \mathrm{mL}$ ), 5,5'-dithiobis(2-nitrobenzoic acid) (DTNB) (0.15 mM) and NADPH (0.2 $\mathrm{mM})$. Change in absorbance after addition of NADPH was monitored at $412 \mathrm{~nm}$ 
for 3 min at $25^{\circ} \mathrm{C}$, using a microtiter plate reader (Enspire® 2300, Perkin-Elmer, Waltham, MA, USA).

Glutathione disulphide was determined after derivatization of GSH with 1-methyl2-vinylpyridinium triflate (M2VP) (Shaik \& Mehvar, 2006). An aliquot (80 $\mu \mathrm{L}$ ) of the GSx sample was incubated with $10 \mu \mathrm{L}$ M2VP (33 mM) and $10 \mu \mathrm{L} 90 \%(\mathrm{v} / \mathrm{v})$ triethanolamine for $5 \mathrm{~min}$ at room temperature. Standard amounts of GSSG (0150 pmol GSSG/20 $\mu \mathrm{L}$ ) were treated the same way, and samples and standards subjected to the same recycling assay as above.

\section{Superoxide dismutase (SOD)}

SOD assays were performed using the riboflavin/nitroblue tetrazolium (RF/NBT) assay in a microtiter plate format (Beauchamp \& Fridovich, 1971, Fryer et al., 1998). Lysate or SOD standard $(20 \mu \mathrm{L})$ was measured in a final reaction mixture of $300 \mu \mathrm{L}$ potassium phosphate buffer $(50 \mathrm{mM}, \mathrm{pH} 7.8)$ containing EDTA (0.1 $\mathrm{mM})$, riboflavin $(1.3 \mu \mathrm{M})$, L-methionine $(10 \mathrm{mM})$, NBT $(57 \mu \mathrm{M})$ and Triton $\mathrm{X}-100$ $(0.025 \%(\mathrm{v} / \mathrm{v}))$. Absorbance was read at $560 \mathrm{~nm}$ both immediately and after 10 min incubation under a homogeneous light field $\left(130 \mu \mathrm{mol}\right.$ quanta $\left.\mathrm{m}^{-2} \mathrm{~s}^{-1}\right)$ at $25^{\circ} \mathrm{C}$ using a microtiter plate reader (Biochrom Anthos 2010 Microplate Reader, Biochrom Ltd., Cambridge, United Kingdom). Standards and samples were measured using the same reaction mixture and a sigmoidal 5-parameter semilogarithmic standard curve (24 standard levels) was used to determine SOD activity of samples. One unit of SOD activity was defined as the amount of enzyme that inhibits the NBT reduction by $50 \%$.

\section{Ascorbate peroxidase (APX)}

Using $100 \mu \mathrm{L}$ lysate in a final reaction mixture of $700 \mu \mathrm{L}$ potassium phosphate buffer $(50 \mathrm{mM}, \mathrm{pH} 7.0)$, EDTA $(0.1 \mathrm{mM})$, ascorbate $(0.3 \mathrm{mM})$ and hydrogen peroxide (0.1 mM) (Nakano \& Asada, 1981), APX activity was assessed by monitoring the oxidation of ascorbate at $290 \mathrm{~nm}$ over $3 \mathrm{~min}$ at $25^{\circ} \mathrm{C}$ (UV-Vis Spectrophotometer UV-2550, Shimadzu Corp., Kyoto, Japan). APX activity was determined with $\varepsilon=2.8 \mathrm{mM}^{-1} \mathrm{~cm}^{-1}$.

\section{Glutathione reductase (GR)}

GR activity was measured according to the DTNB method, using $20 \mu \mathrm{L}$ lysate in a final reaction mixture of $200 \mu \mathrm{L}$, containing sodium phosphate buffer (100 mM, 
$\mathrm{pH}$ 7.5), EDTA (1 mM), DTNB (0.75 mM), NADPH (0.1 mM) and GSSG (1 mM) (Smith et al., 1988, Cribb et al., 1989). Increase in absorbance at $412 \mathrm{~nm}$ over 3 min at $25^{\circ} \mathrm{C}$ was monitored (Enspire ${ }^{\circledR}$ 2300, Perkin-Elmer, Waltham, MA, USA) and GR activity calculated $\left(\varepsilon=14.151 \mathrm{mM}^{-1} \mathrm{~cm}^{-1}\right.$ with $\left.\mathrm{d}=0.571 \mathrm{~cm}\right)$.

\section{Glutathione S-transferase (GST)}

GST activity was determined via GSH conjugation with 1-chloro-2,4dinitrobenzene (CDNB) at $340 \mathrm{~nm}$ over $3 \mathrm{~min}$ at $25^{\circ} \mathrm{C}$ in a microtiter plate format (Habig et al., 1974). A $20 \mu \mathrm{L}$ aliquot of lysate was incubated in a final reaction mixture of $200 \mu \mathrm{L}$, containing sodium phosphate buffer (100 mM, pH 6.5), CDNB $(1 \mathrm{mM})$ and GSH $(2 \mathrm{mM})$, and GST activity calculated $\left(\varepsilon=9.6 \mathrm{mM}^{-1} \mathrm{~cm}^{-1}\right.$ with $\mathrm{d}=$ $0.571 \mathrm{~cm})$.

All enzyme activities were normalized to protein content and expressed as specific activity $\left(\mathrm{U} \mathrm{mg}^{-1}\right)$, after checking that protein content per cell did not significantly change in any of the treatments.

\section{Statistical analysis}

PAM data were arcsine square root transformed and analysed for 'time', 'type' and 'temperature' effects using a repeated measures analysis of variance (rmANOVA). Normality of data was confirmed with the Shapiro-Wilk test and sphericity assumptions tested using Mauchly's sphericity test. In case sphericity was violated, results of Pillai's trace test or the epsilon-adjusted univariate F-test (Greenhouse-Geisser; G-G) at a confidence level of 0.05 are reported. For chlorophyll and enzyme data, multivariate analysis of variance (MANOVA) on linear slopes over time was performed due to missing data points (death or nondetectable levels). Significant effects are reported for the overall dataset (pooled type data) using the factors 'type', 'temperature' and 'type $x$ temperature', as well as for individual types using ANOVA to test for temperature effects (with post hoc Tukey HSD test). In some cases, rmANOVA was used to investigate the relationship between temperature and time effects for specific aspects of the results. All datasets were checked for significant autocorrelation using the DurbinWatson test.

Comparison of antioxidant baseline levels at $25^{\circ} \mathrm{C}$ between types was done via ANOVA or Welch ANOVA with post hoc Tukey HSD tests for Day 0 data. A linear 
discriminant function analysis (DFA) was performed to test how well baseline activities of a set of antioxidants would delineate Symbiodinium genotypes, using the complete $25^{\circ} \mathrm{C}$ dataset over 14 days to account for the natural variation. Principal components analysis (PCA), using a correlation matrix, was performed to visualize the antioxidant network response for each genotype under the temperatures tested. For missing values, the implemented imputation method of JMP was employed and score plots with imputation are shown. For DFA and PCA, only Eigenvalues larger than one were considered. Data were analysed using JMP 10.0.0 (SAS Institute Inc., Cary, NC, USA).

\section{Results}

\section{Cell viability}

PSIl fluorescence yields showed significant differences in $F_{v} / F_{m}$ response, based on all main factors (type, temperature, time) and their interaction (rmANOVA, $\mathrm{p}<0.001$ ). While Symbiodinium $\mathrm{F} 1$ survived at $33^{\circ} \mathrm{C}$ for 14 days without a decline in growth or $F_{v} / F_{m}$, all other types died within the second week, with $F_{v} / F_{m}$ values on Day 7 at $33^{\circ} \mathrm{C}$ being $29 \%$ (B1), 30\% (C1) and $45 \%$ (E) of initial values (Figure 2.3). No differences in the rate of decline for $F_{v} / F_{m}$ at $33^{\circ} \mathrm{C}$ were found $\left(\right.$ rmANOVA; $\mathrm{B} 1=\mathrm{C} 1=\mathrm{E}$; Days 0 to 7 at $33^{\circ} \mathrm{C}$ ). A temperature of $29^{\circ} \mathrm{C}$ had no significant effect on growth or $\mathrm{F}_{\mathrm{v}} / \mathrm{F}_{\mathrm{m}}$ in the Symbiodinium cultures, except for $\mathrm{B} 1$ where growth was significantly reduced by $35 \%$ during the second week (Wilcoxon test, $x^{2}=5.6333, p=0.0176$ ) (Figure 2.3 A). Chl a content $\left(p g\right.$ cell $^{-1}$ ) on Day 0 was significantly different between each type, with Symbiodinium E (3.61 \pm $0.21)>\mathrm{F} 1(2.46 \pm 0.25)>\mathrm{B} 1(1.14 \pm 0.18)>\mathrm{C} 1(0.30 \pm 0.22)$ (Welch ANOVA, $F_{3}$, $20.934=102.499, p<0.0001)$. A significant decline in chl a level relative to that at $25^{\circ} \mathrm{C}$ was only observed for $\mathrm{B} 1$ at $33^{\circ} \mathrm{C}$, where levels decreased by $21 \%$ over the first week $\left(F_{2,2}=15.525, \mathrm{p}=0.0004\right.$, Appendix Figure 7.1). 

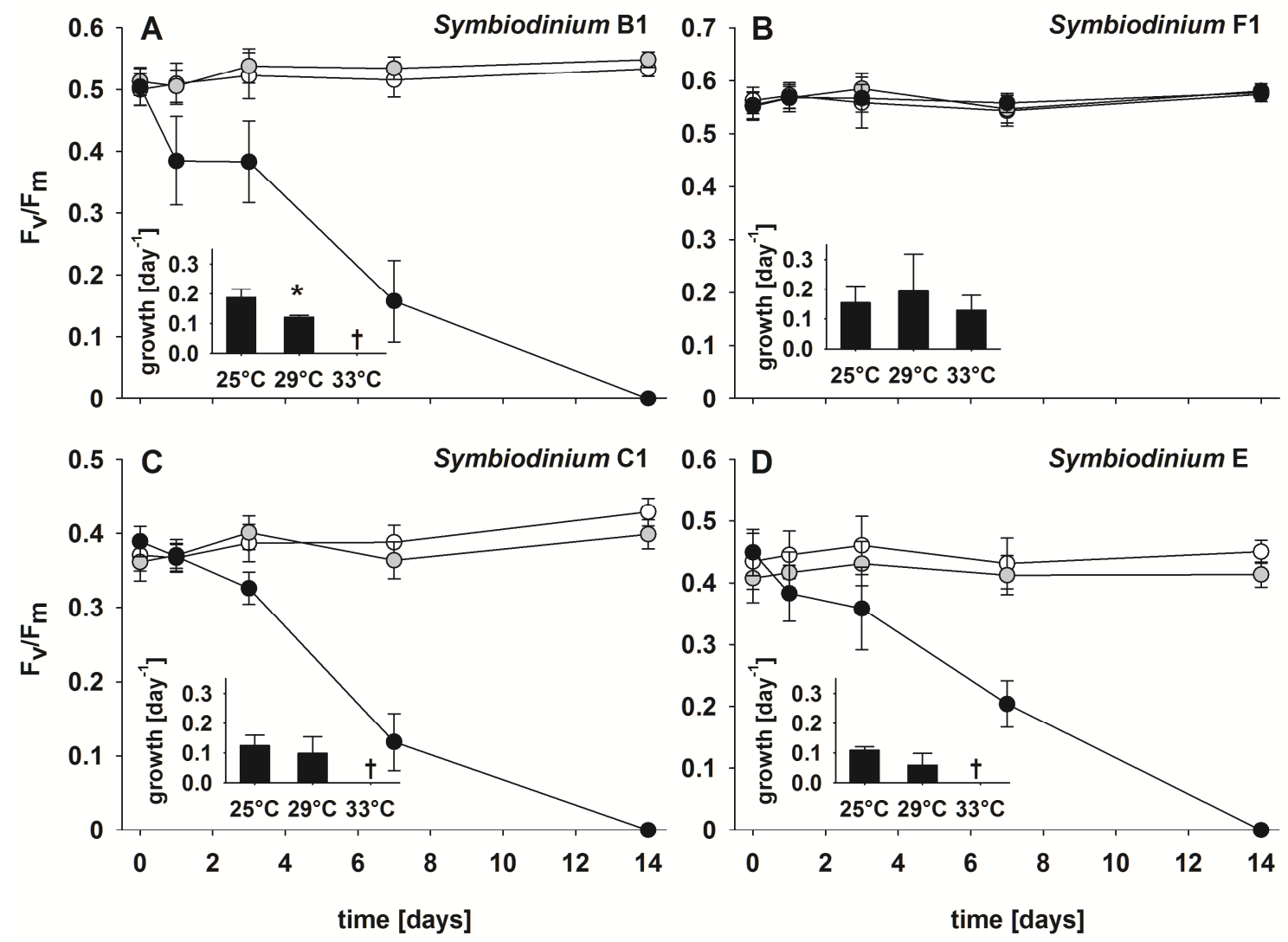

Figure 2.3. Viability of Symbiodinium types B1, F1, C1 and E.

Shown are maximum quantum yields and growth rates of Symbiodinium genotypes B1 (A), $F 1$ (B), $C 1$ (C) and $E(D)$ over time in response to three thermal treatments. Maximum quantum yields $\left(\mathrm{F}_{\mathrm{v}} / \mathrm{F}_{\mathrm{m}}\right)$ at $25^{\circ} \mathrm{C}$ (white), $29^{\circ} \mathrm{C}$ (light grey) and $33^{\circ} \mathrm{C}$ (black) shown as interpolated scatter plots, with cell growth rates [day ${ }^{-1}$ ] between Days 7 and 14 at each temperature shown as bar graphs (insert). Significant differences of growth rates $(p<0.05)$ between treatments are indicated by asterisks, and negative growth rates with subsequent cell death are indicated by daggers. Values are means \pm SE; Symbiodinium B1 $(\mathrm{N}=5-6), \mathrm{C} 1(\mathrm{~N}=3-4), \mathrm{E}(\mathrm{N}=4), \mathrm{F} 1(\mathrm{~N}=4-5)$.

\section{Baseline antioxidants levels}

Constitutive levels of antioxidants at $25^{\circ} \mathrm{C}$ varied significantly between all Symbiodinium genotypes (Table 2.1). Differences were largest for glutathione content and GST activity. Type C1 had a fivefold larger glutathione pool, than all other types and GST activity in B1 was an order of magnitude smaller than in $\mathrm{E}$ and F1. A discriminant function analysis based on their constitutive antioxidant features was able to identify all four Symbiodinium genotypes (Figure 2.4; Pillai's trace, $\left.F_{15,159}=24.207, p<0.0001\right)$. Separation of types along discriminant function 1 was driven by GR, GSx and GST, while along discriminant function 2, GSX and APX were most prominent. The analysis clearly delineated separate 
clusters for Symbiodinium B1, C1 and an overlapping cluster of E and F1 (Figure 2.4). SOD activity mainly discriminated along a third function, explaining the remaining variation. While the cluster of $E$ and $F 1$ visually overlapped, the statistical prediction was accurate enough to separate these two types from one another (misclassification of $E$ in predicted $F 1$ was 33\%; F1 in predicted $E$ was 7\%). Baseline values were most similar for SOD activity, and baseline variability between types increased in the order APX $<$ GR $<$ GST $<$ GSx (based on the ratio between range and mean, Table 2.1; visible as relative length of biplot rays; Figure 2.4).

Table 2.1. Baseline levels of antioxidants in Symbiodinium.

Shown are constitutive levels and activities of antioxidants in different Symbiodinium ITS2 types under control conditions on Day $0\left(25^{\circ} \mathrm{C}, 40-50 \mu \mathrm{mol}\right.$ quanta $\left.\mathrm{m}^{-2} \mathrm{~s}^{-1}\right)$. Superoxide dismutase (SOD; $N=9-16$ ), ascorbate peroxidase (APX; $N=9-16$ ), glutathione content (GSx; $N=10-13$ ), glutathione reductase (GR; $N=11-16$ ), glutathione $\mathrm{S}$-transferase (GST; $\mathrm{N}=12-15$ ). All values are normalized to protein content either as specific activity ( $\mathrm{U} \mathrm{mg}^{-1}$; SOD, APX, GR, GST) or amount (nmol $\mathrm{mg}^{-1}$; GSX) and expressed as means \pm SE. Probability values are based on ANOVA or Welch ANOVA $\left(^{*}\right)$ tests between types. Types not connected by the same letter within each column are significantly different based on post-hoc Tukey HSD test. Range/mean ratios were calculated from the shown means of each type.

\begin{tabular}{cccccc}
$\begin{array}{c}\text { ITS2 } \\
\text { genotype }\end{array}$ & $\begin{array}{c}\text { SOD } \\
\mathrm{p}<0.0014^{*}\end{array}$ & $\begin{array}{c}\text { APX } \\
\mathrm{p}<0.0001^{*}\end{array}$ & $\begin{array}{c}\text { GSx } \\
\mathrm{p}<0.0001\end{array}$ & $\begin{array}{c}\text { GR } \\
\mathrm{p}<0.0001^{*}\end{array}$ & $\begin{array}{c}\text { GST } \\
\mathrm{p}<0.0001\end{array}$ \\
\hline $\mathrm{B} 1$ & $80.31 \pm 5.43^{\mathrm{b}}$ & $0.50 \pm 0.04^{\mathrm{c}}$ & $64.62 \pm 7.49^{\mathrm{a}}$ & $0.40 \pm 0.03^{\mathrm{a}}$ & $0.037 \pm 0.008^{\mathrm{c}}$ \\
$\mathrm{C} 1$ & $82.85 \pm 3.75^{\mathrm{a}, \mathrm{b}}$ & $0.83 \pm 0.08^{\mathrm{b}}$ & $319.60 \pm 32.77^{\mathrm{b}}$ & $0.13 \pm 0.03^{\mathrm{b}}$ & $0.14 \pm 0.03^{\mathrm{b}}$ \\
$\mathrm{E}$ & $63.14 \pm 7.18^{\mathrm{b}}$ & $0.77 \pm 0.05^{\mathrm{b}}$ & $75.30 \pm 8.11^{\mathrm{a}}$ & $0.13 \pm 0.01^{\mathrm{b}}$ & $0.48 \pm 0.06^{\mathrm{a}}$ \\
$\mathrm{F} 1$ & $106.75 \pm 6.91^{\mathrm{a}}$ & $1.44 \pm 0.05^{\mathrm{a}}$ & $68.49 \pm 5.80^{\mathrm{a}}$ & $0.15 \pm 0.02^{\mathrm{b}}$ & $0.50 \pm 0.06^{\mathrm{a}}$ \\
\hline $\begin{array}{c}\text { Range/ } \\
\text { mean ratio }\end{array}$ & 0.52 & 1.05 & 1.93 & 1.32 & 1.61
\end{tabular}




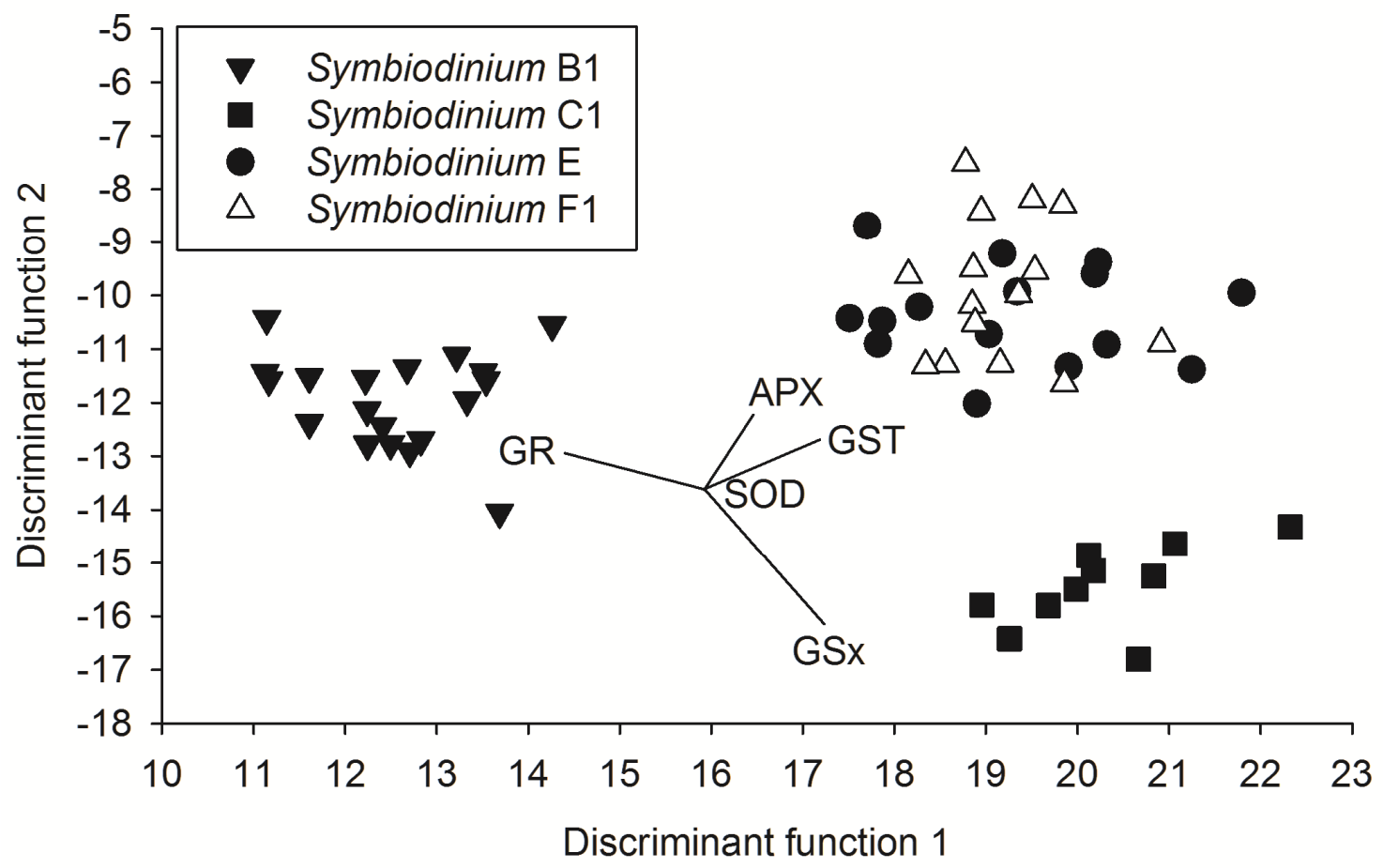

Figure 2.4. Type-specific antioxidant profiles in Symbiodinium.

Linear discriminant analyses of Symbiodinium types based on antioxidant levels at $25^{\circ} \mathrm{C}$, integrating enzymatic activities of superoxide dismutase (SOD), ascorbate peroxidase (APX), glutathione reductase (GR), glutathione S-transferase (GST) and the glutathione pool size (GSx) over two weeks. Values are canonical scores for two discriminant functions that explain a cumulative variation of $96.4 \%$. Biplot rays indicate the coordinate direction for each antioxidant. SOD is directed into the plane and mainly discriminates along a third function.

Changes in all antioxidants, except GST, were significantly affected by temperature, and these temperature effects were type specific for the responses of GSx and GR (Table 2.2). While differing trends in APX, GST and GSH/GSx values for some types and temperatures were observed, these effects were not significant in the overall dataset (Table 2.2). 
Table 2.2. Statistical effects of temperature and Symbiodinium type for specific antioxidants.

Shown are MANOVA test results on changes over time, based on the overall dataset (all types together) over 14 days. Significant effects are indicated by asterisks. Superoxide dismutase (SOD), ascorbate peroxidase (APX), glutathione pool size (GSx), reduced glutathione content relative to total glutathione (GSH/GSx), glutathione reductase (GR), glutathione S-transferase (GST).

\begin{tabular}{cccc} 
Variable & Type & Temperature & \multicolumn{1}{c}{ Type x Temperature } \\
\hline SOD & $F_{3,3}=0.052, \mathrm{p}=0.9841$ & $F_{2,2}=18.659, \mathrm{p}<0.0001^{*}$ & $F_{6,6}=2.119, \mathrm{p}=0.0766$ \\
APX & $F_{3,3}=1.481, \mathrm{p}=0.2351$ & $F_{2,2}=11.265, \mathrm{p}=0.0001^{*}$ & $F_{6,6}=1.679, \mathrm{p}=0.1530$ \\
GSx & $F_{3,3}=0.951, \mathrm{p}=0.4275$ & $F_{2,2}=15.646, \mathrm{p}<0.0001^{*}$ & $F_{6,6}=2.569, \mathrm{p}=0.0381^{*}$ \\
GSH/GSx & $F_{3,3}=0.505, \mathrm{p}=0.6818$ & $F_{2,2}=25.172, \mathrm{p}<0.0001$ & $F_{6,6}=2.245, \mathrm{p}=0.0614$ \\
GR & $F_{3,3}=2.488, \mathrm{p}=0.0735$ & $F_{2,2}=4.344, \mathrm{p}=0.0193^{*}$ & $F_{6,6}=9.479, \mathrm{p}<0.0001^{*}$ \\
GST & $F_{3,3}=2.270, \mathrm{p}=0.0951$ & $F_{2,2}=0.044, \mathrm{p}=0.9572$ & $F_{6,6}=1.033, \mathrm{p}=0.4185$
\end{tabular}

\section{Antioxidant response in Symbiodinium B1}

Activity of SOD was only significantly upregulated at $33^{\circ} \mathrm{C}$ compared to $25^{\circ} \mathrm{C}$ and $29^{\circ} \mathrm{C}$ (SOD: $F_{2,2}=16.678, \mathrm{p}=0.0003$ ). Elevated enzyme activities were detected on Day 3 and increased thereafter, with maximum activities of SOD observed on Day $7(165 \pm 14 \%$ of Day 0 value; Figure $2.5 \mathrm{~A})$. As for SOD, APX activity at $33^{\circ} \mathrm{C}$ was significantly upregulated (APX: $F_{2,2}=12.953, p=0.0008$ ), reaching its maximum on Day 7 (237 $\pm 27 \%$; Figure $2.5 \mathrm{~B})$. APX activity at $29^{\circ} \mathrm{C}$ was only temporarily elevated on Day 7 (Welch ANOVA post hoc Tukey HSD, $F_{2,4.075=}$ 8.912, $p=0.0325)$, but returned to control levels by Day 14 (Figure 2.5B). Activities of SOD and APX were significantly, and positively correlated at $29^{\circ} \mathrm{C}(r$ $=0.49, \mathrm{p}=0.0116, \mathrm{~N}=26)$ and $33^{\circ} \mathrm{C}(\mathrm{r}=0.67, \mathrm{p}=0.0024, \mathrm{~N}=18)$.

The total glutathione content per unit of protein (GSx) at $29^{\circ} \mathrm{C}$ increased until Day 7 , when the concentration reached $145 \pm 14.9 \mathrm{nmol} \mathrm{mg}^{-1}$ protein, before returning to control levels by Day 14 (Figure 2.6; rmANOVA with $25^{\circ} \mathrm{C}, 29^{\circ} \mathrm{C}$ over 14 days, $\left.F_{\text {Time } x \text { Temp(4,4) }}=9.008, p=0.028\right)$. No change in the redox state of glutathione was observed for this temperature. At $33^{\circ} \mathrm{C}$, significant increases in GSx were observed in Symbiodinium B1 $\left(F_{2,2}=36.022\right.$, $\left.p<0.0001\right)$ and GSx levels peaked

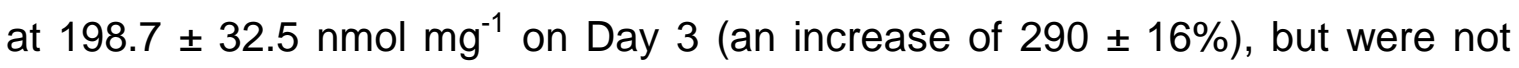
detectable on Day 7. As GSx increased at $33^{\circ} \mathrm{C}$, a significant drop by 23 percentage points was observed in the GSH/GSx ratio within the first three days (Figure 2.6B; $F_{2,2}=11.545, p=0.0025$ ). 
GR activities tended to increase at $29^{\circ} \mathrm{C}$, but declined by $50 \%$ from their initial value $\left(0.43 \pm 0.06 \mathrm{U} \mathrm{mg}^{-1}\right)$ after seven days at $33^{\circ} \mathrm{C}$ (Figure 2.7A). A significant difference was observed between the $29^{\circ} \mathrm{C}$ and $33^{\circ} \mathrm{C}$ treatments on Day 7 (ANOVA post hoc Tukey HSD, $F_{2,11}=8.818, p=0.0052$ ), though both were not significantly different from the control. Despite the declines of GSH/GSx and GR at $33^{\circ} \mathrm{C}$, increases in total glutathione allowed for significantly higher GSH concentrations at $33^{\circ} \mathrm{C}\left(245.3 \pm 30.7 \mathrm{nmol} \mathrm{GSH} \mathrm{mg}{ }^{-1}\right)$ compared to $25^{\circ} \mathrm{C}$ on Day 3 (ANOVA, $F_{2,9}=4.5216, p=0.0437$ ). Thus, the effective $\mathrm{GSH}$ concentration at $33^{\circ} \mathrm{C}$ on Day 3 corresponded to maximum GSH concentrations observed at $29^{\circ} \mathrm{C}$ on Day $7\left(270.1 \pm 29.2 \mathrm{nmol} \mathrm{GSH} \mathrm{mg}{ }^{-1}\right)$.

Symbiodinium B1 was the only type that exhibited elevated GST activity in response to $33^{\circ} \mathrm{C}$, as it increased significantly from $0.040 \pm 0.009 \mathrm{U} \mathrm{mg}^{-1}$ at Day 0 to $0.070 \pm 0.014 \mathrm{U} \mathrm{mg}^{-1}$ at Day 7 (Figure 2.7B, $F_{2,2}=5.438, p=0.0228$ ). 

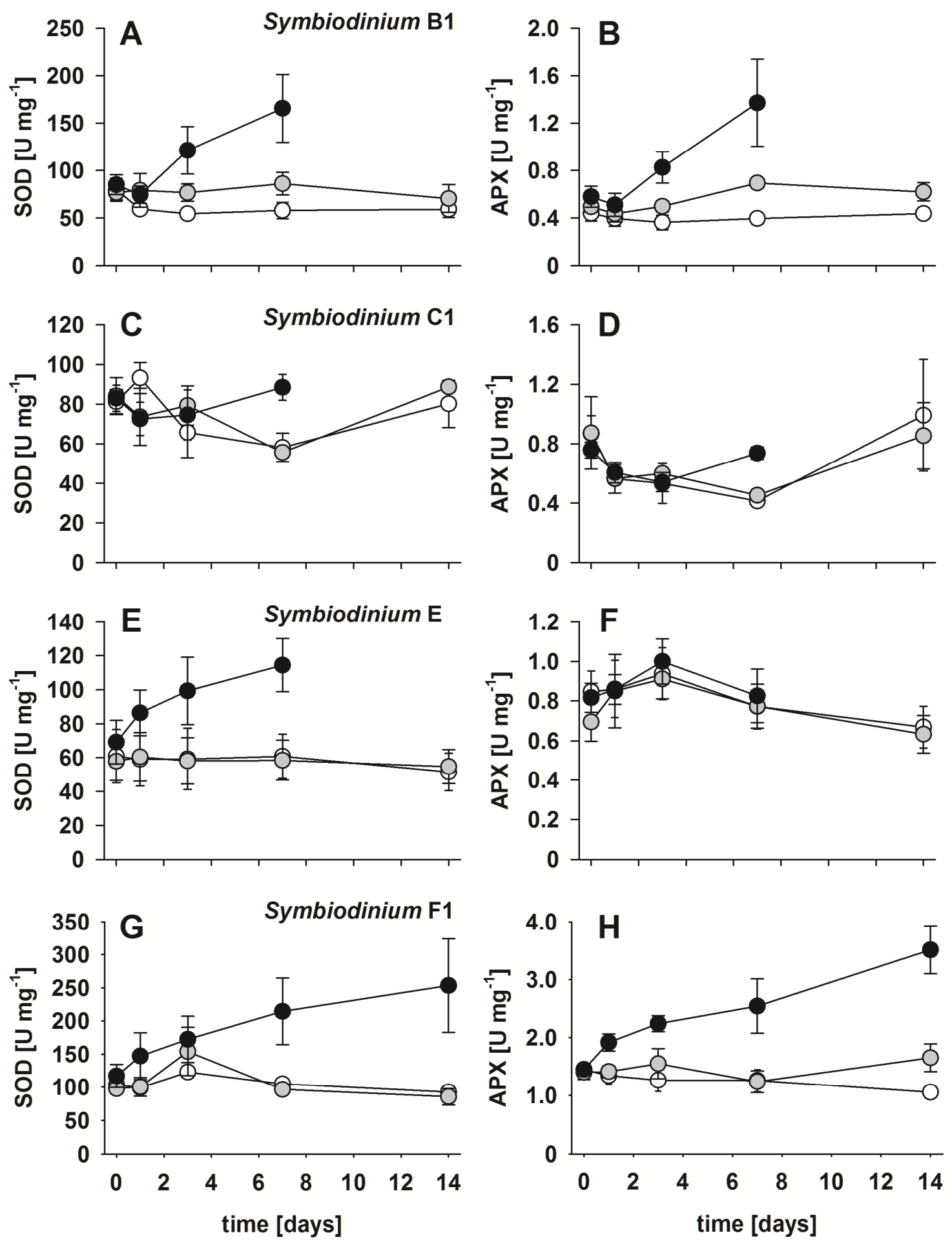

Figure 2.5. SOD-APX pathway activity.

Superoxide dismutase (SOD, left) and ascorbate peroxidase (APX, right) activity as units per mg protein of Symbiodinium types B1 (A-B), C1 (C-D), E (E-F) and F1 (G-H) over 14 days at $25^{\circ} \mathrm{C}$ (white), $29^{\circ} \mathrm{C}$ (grey) and $33^{\circ} \mathrm{C}$ (black), shown as interpolated scatter plots. Symbiodinium B1, $\mathrm{C} 1$ and $\mathrm{E}$ died after seven days at the highest temperature. Values are means \pm SE. Symbiodinium B1 $(N=5-6), C 1(N=3), E(N=4-5), F 1(N=4-5)$. Note different scaling of axes. 

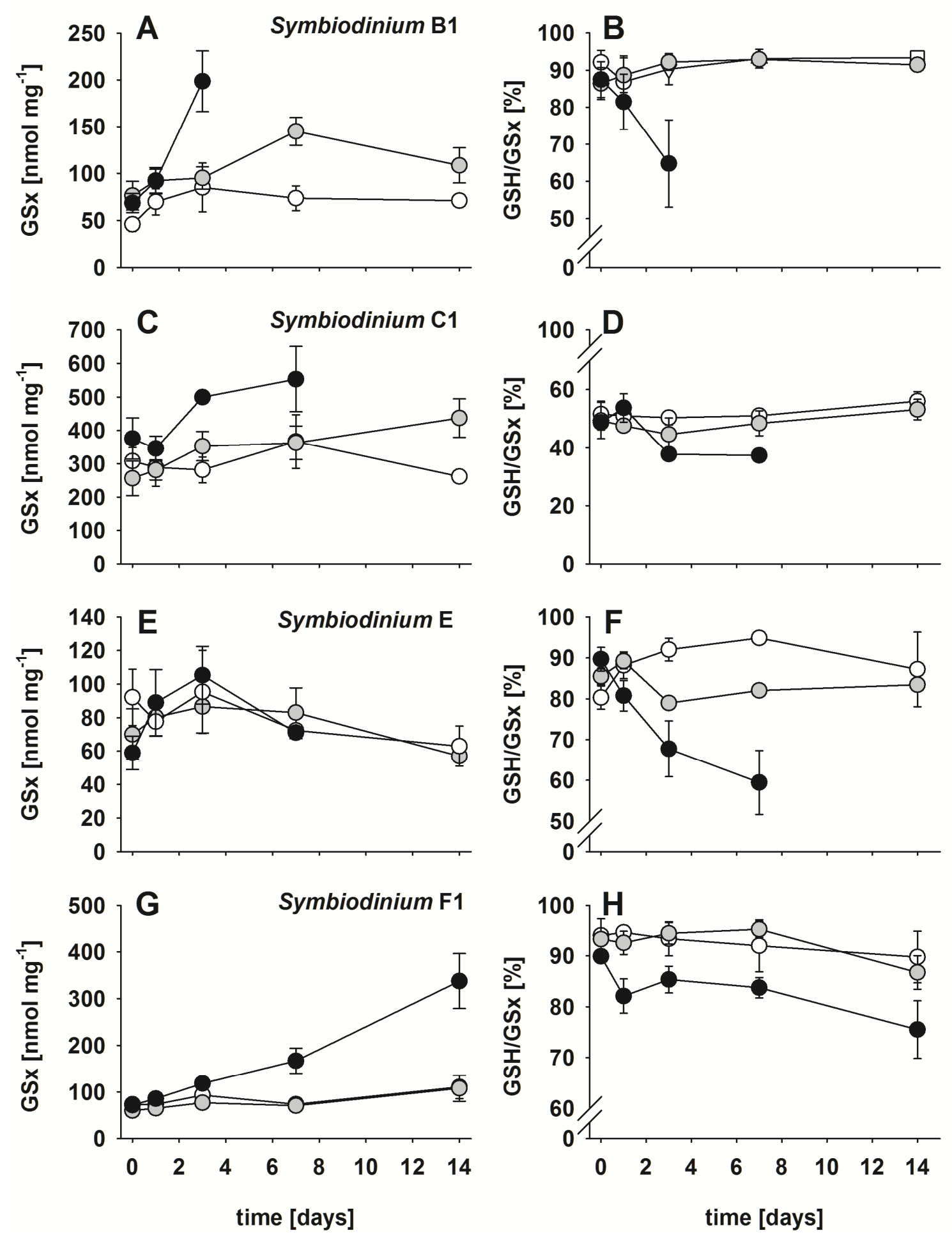

Figure 2.6. Size and redox state of the glutathione pool.

Glutathione pool size (left; GSx; nmol per mg protein) and corresponding redox state (right; GSH/GSx) for Symbiodinium types B1 (A-B), C1 (C-D), E (E-F) and F1 (G-H) at $25^{\circ} \mathrm{C}$ (white), $29^{\circ} \mathrm{C}$ (grey) and $33^{\circ} \mathrm{C}$ (black) over 14 days of exposure, shown as interpolated scatter plots. Symbiodinium B1, C1 and $\mathrm{E}$ died after seven days at $33^{\circ} \mathrm{C}$. Values are means \pm SE. Symbiodinium B1 $(N=4-5), C 1, E$ and $F 1$ (all $N=3-4)$. Note different scaling of axes. 

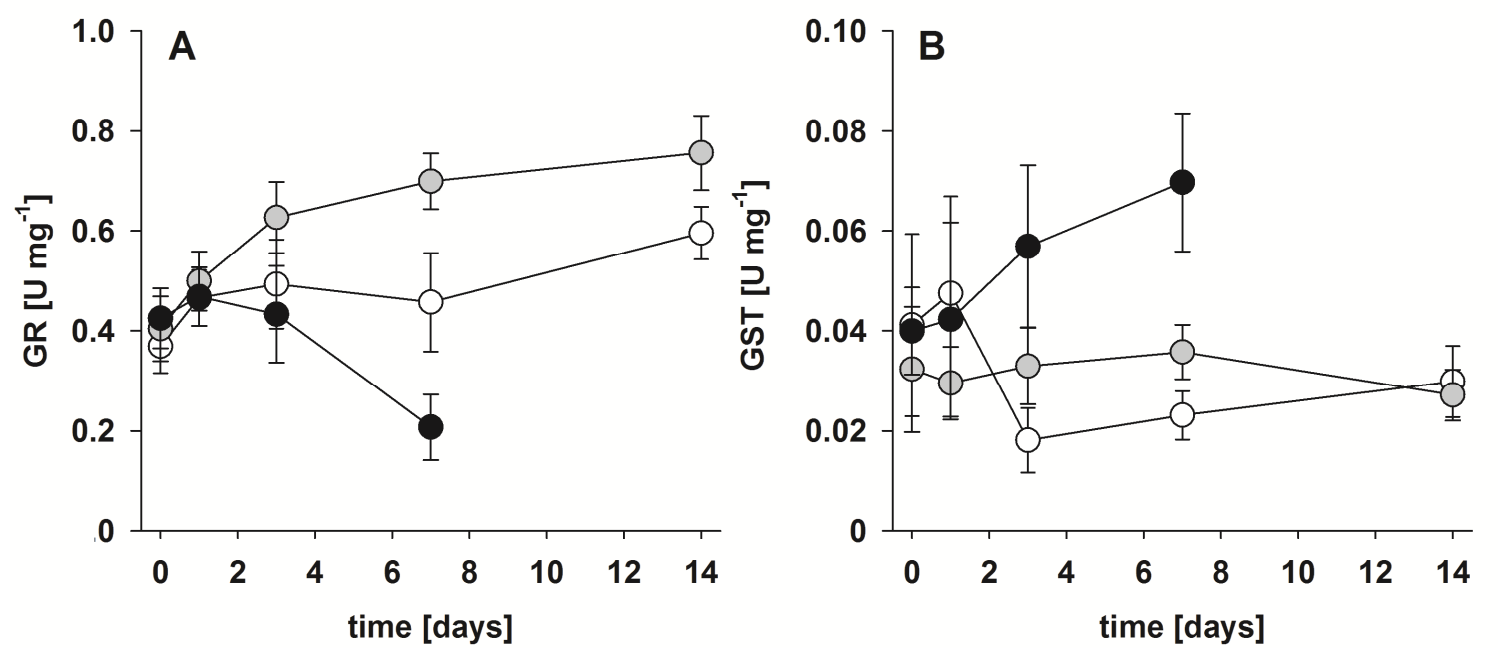

Figure 2.7. GR and GST activity in Symbiodinium B1.

Activity of glutathione reductase (GR; A) and glutathione S-transferase (GST; B) in units per mg protein at $25^{\circ} \mathrm{C}$ (white), $29^{\circ} \mathrm{C}$ (grey) and $33^{\circ} \mathrm{C}$ (black) in Symbiodinium B1 over 14 days of exposure, shown as interpolated scatter plots. Culture died after seven days at $33^{\circ} \mathrm{C}$. Values are means $\pm \mathrm{SE}(\mathrm{N}=5-6)$.

\section{Antioxidant response in Symbiodinium C1}

No significant change in any of the antioxidants was observed in Symbiodinium $\mathrm{C} 1$ at $29^{\circ} \mathrm{C}$. However, at $33^{\circ} \mathrm{C}$ the activities of SOD and APX increased by Day 7 (SOD: $F_{2,6}=9.965, \mathrm{p}=0.0124 ;$ APX: $F_{2,6}=27.182, \mathrm{p}=0.0010$ ), to $88.52 \pm 6.55$ $\mathrm{U} \mathrm{mg}^{-1}$ and $0.74 \pm 0.04 \mathrm{U} \mathrm{mg}^{-1}$, respectively (corresponding to $152 \%$ and $178 \%$ of the control level; Figure 2.5C-D). SOD and APX activities were positively correlated in all treatments, with the strength of this correlation remaining similar, irrespective of temperature $(r=0.58, p<0.0001, N=42)$.

Symbiodinium C1 exhibited a larger glutathione pool (GSx mg-1 protein), but a much lower glutathione redox state than the other types (GSH/GSx values of approximately $50 \%$ ). No significant change of the glutathione content in response to temperature was observed $\left(F_{2,2}=4.096, \mathrm{p}=0.0596\right)$, though the glutathione pool size tended to increase at $33^{\circ} \mathrm{C}$ and peaked at $553.2 \pm 97.5 \mathrm{nmol} \mathrm{mg}^{-1}$ (an increase of $147 \pm 18 \%$ ) on Day 7 (Figure 2.6C). As glutathione levels increased at $33^{\circ} \mathrm{C}$, the amount of reduced glutathione relative to the total glutathione pool significantly declined by 11 percentage points $\left(F_{2,2}=6.177, \mathrm{p}=0.0239\right.$, Fig. 5D). No significant changes in either GR or GST were observed. 


\section{Antioxidant response in Symbiodinium $E$}

A significant increase in SOD activity over time was only found for the highest temperature in Symbiodinium E $\left(F_{2,2}=14.782, \mathrm{p}=0.0014\right)$. SOD activity continuously increased from $69.16 \pm 12.76 \mathrm{U} \mathrm{mg}^{-1}$ on Day 0 to $114.38 \pm 15.63 \mathrm{U}$ $\mathrm{mg}^{-1}$ on Day 7 (an increase of $165 \pm 14 \%$ ) (Figure 2.5E). Neither APX activity nor glutathione pool size was affected by any temperature increase in Symbiodinium E (Figure $2.6 \mathrm{E}$ ). However, at $33^{\circ} \mathrm{C}, \mathrm{GSH} / \mathrm{GSx}$ values significantly declined by 30 percentage points from their initial value of $89.7 \pm 2.9 \%$ between Days 0 and 7 $\left(F_{2,2}=17.701, \mathrm{p}=0.0031\right)$ (Figure $\left.2.6 \mathrm{~F}\right)$. GR and GST activity did not significantly change at either $29^{\circ} \mathrm{C}$ or $33^{\circ} \mathrm{C}$.

\section{Antioxidant response in Symbiodinium $\mathrm{F1}$}

Neither SOD nor APX activity was significantly different from the control levels at $29^{\circ} \mathrm{C}$ in Symbiodinium F1. Though SOD activity doubled between Days 0 and 3 at $33^{\circ} \mathrm{C}$ and continued to rise to $253.5 \pm 70.5 \mathrm{U} \mathrm{mg}^{-1}$ on Day 14 (an increase of $217 \pm 28 \%$ ), the variation between experimental replicates meant that no overall significant effect of temperature on SOD activity was detected (Figure 2.5G). Activity of $\mathrm{APX}$ on the other hand, significantly increased at $33^{\circ} \mathrm{C}\left(F_{2,2}=7.498, \mathrm{p}\right.$ $=0.0102$ ), reaching its maximum value of $3.51 \pm 0.41 \mathrm{U} \mathrm{mg}^{-1}$ on Day 14 (an increase of $242 \pm 12 \%$ ) (Figure $2.5 \mathrm{H}$ ). A significant positive correlation between SOD and APX activity was only found at $33^{\circ} \mathrm{C}(r=0.74, p<0.0001, \mathrm{~N}=25)$.

Over the course of the experiment, elevated GSx levels were observed only at $33^{\circ} \mathrm{C}$ (Figure 2.6G; $F_{2,2}=8.3679, \mathrm{p}=0.0139$ ) with maximum concentrations of $337.1 \pm 58.8 \mathrm{nmol} \mathrm{mg}{ }^{-1}$ on Day 14 (an increase of $471 \pm 17 \%$ ). Statistically, GSH/GSx ratios were not affected by temperature over the duration of the experiment. However, the overall GSH/GSx ratio was significantly lower at $33^{\circ} \mathrm{C}$ $\left(\mathrm{rmANOVA}, F_{\text {Temp }(2,7)}=6.573, \mathrm{p}=0.0247 ; F_{\text {Time }(4,4)}=0.087 ; F_{\text {Time }} \times \operatorname{Temp(8,10)}=\right.$ $0.862, p=0.575$, Figure $2.6 \mathrm{H}$ ). GR activity did not change during the first week in any of the treatments, but reached $0.41 \pm 0.06 \mathrm{U} \mathrm{mg}^{-1}$ by Day 14 at $33^{\circ} \mathrm{C}$ and was therefore about twice the control value (ANOVA, $F_{2,10}=4.824, \mathrm{p}=0.0341$, Appendix Figure 7.3). No changes in GST activity were observed in any of the treatments. 


\section{Network responses in Symbiodinium genotypes}

Breaking down all the antioxidant responses into two principal components (two sets of values that account for the majority of the variance) illustrated the main activity shifts in the antioxidant network in response to temperature in the Symbiodinium genotypes. Although cumulative variation explained by the first two principal components was similar for all types with $72.4 \%$ for $\mathrm{B} 1,69.3 \%$ for $\mathrm{C} 1$, $73.2 \%$ for $E$ and $79.8 \%$ for $F 1$, a clear thermal or temporal response could only be identified for Symbiodinium B1 and F1 (Figure 2.8).

For Symbiodinium B1, SOD and APX activity, and GST activity and glutathione pool size, were correlated and constituted PC1. GR activity was the main vector in PC2. At $29^{\circ} \mathrm{C}$, the main network shift occurred along PC2 with increased GR activity after Day 3 with a slight positive movement along PC1 (Figure 2.8). For $33^{\circ} \mathrm{C}$, replicates of Day 3 and 7 were characterized by lower GR activity and higher activity of SOD/APX and GST/GSx, compared to the $29^{\circ} \mathrm{C}$ treatment (Figure 2.8).

For Symbiodinium F1, the main shift was observed along PC1, consisting of SOD and APX vectors grouped closely together, as well as separate vectors for GR and GSx. GST and also GSx contributed to PC2. While no change in the network of this Symbiodinium genotype was observed at $29^{\circ} \mathrm{C}$, it shifted along $\mathrm{PC} 1$ and in the direction of the GSx vector during Days 3 and 7 at $33^{\circ} \mathrm{C}$. After 14 days at $33^{\circ} \mathrm{C}$, a small cluster containing four of the five replicates can be seen, characterized by high score values for SOD, APX and GSx (Figure 2.8). 
Symbiodinium B1

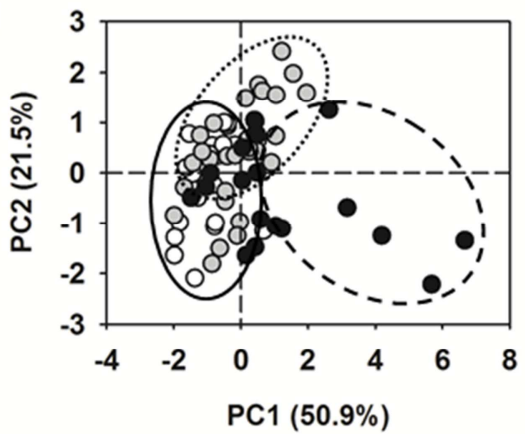

Symbiodinium $\mathrm{C} 1$

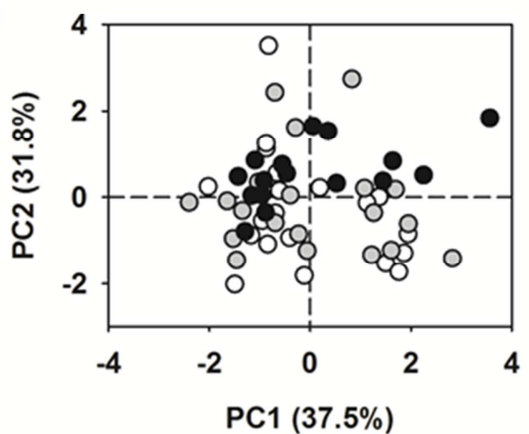

Symbiodinium $\mathrm{E}$

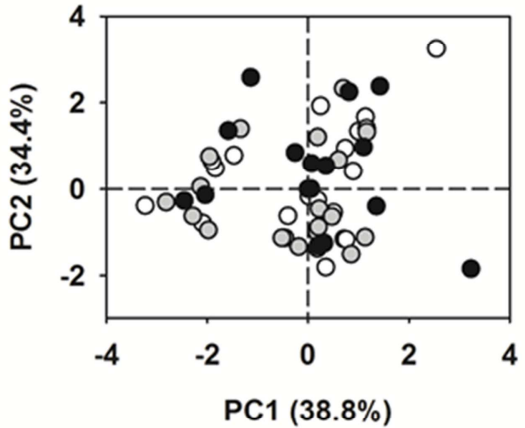

Symbiodinium F1

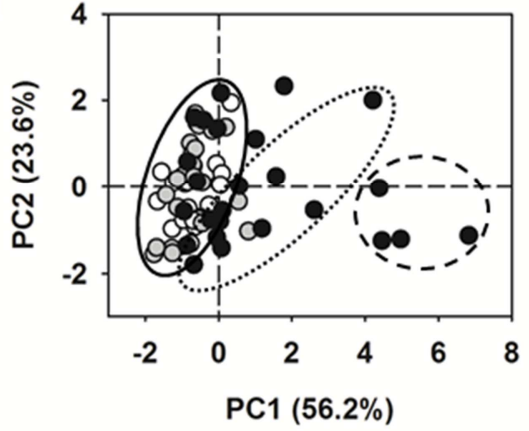

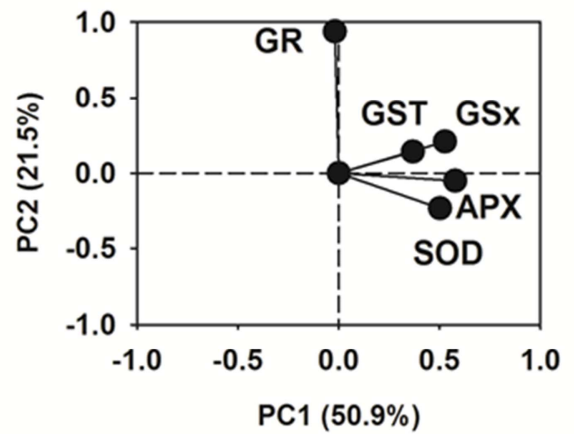
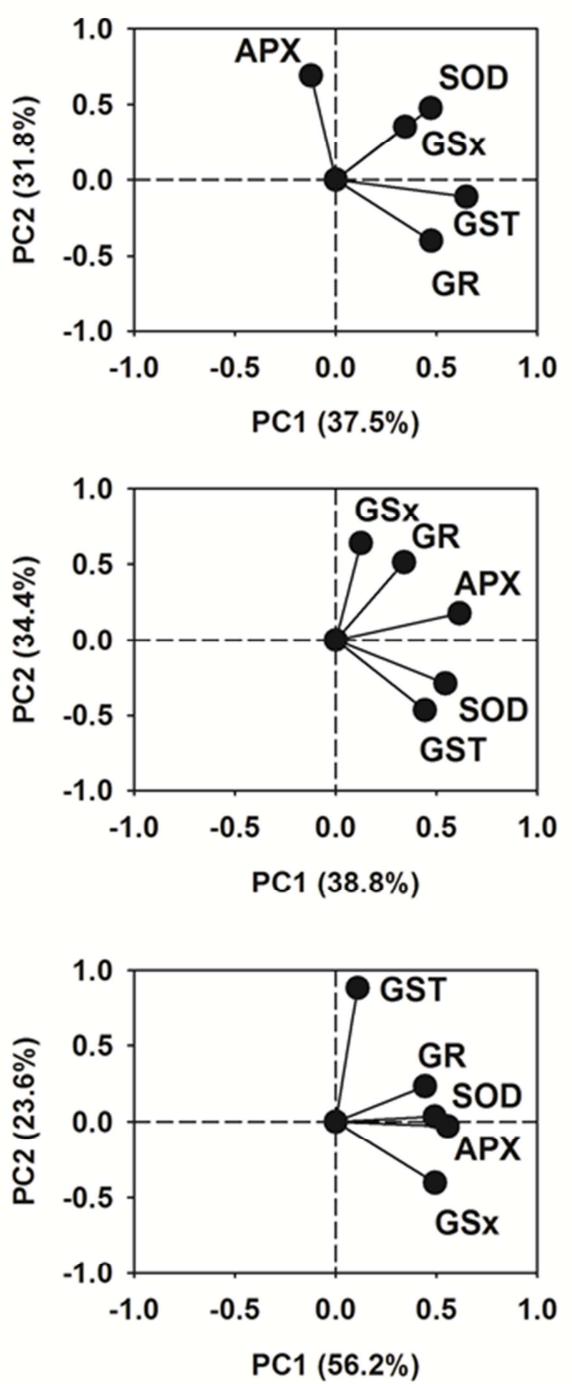

Figure 2.8. PCA analysis of antioxidant responses.

Principal component analysis of antioxidant responses, integrating 14 days of exposure to $25^{\circ} \mathrm{C}$ (white), $29^{\circ} \mathrm{C}$ (grey) and $33^{\circ} \mathrm{C}$ (black) in four different Symbiodinium types. Score plots (left) and corresponding loading plots with Eigenvectors (right) are shown for the first two principal components (PC1 and PC2). Ovals indicate main network shifts under treatments, containing all or the majority of replicates. For Symbiodinium B1: Replicates for the control treatment, and Days 0 and 1 for $29^{\circ} \mathrm{C}$ and $33^{\circ} \mathrm{C}$, are enclosed by the solid oval; replicates for Days 3-14 at $29^{\circ} \mathrm{C}$ (dotted oval); and replicates for Days $3-7$ at $33^{\circ} \mathrm{C}$ (dashed oval). For Symbiodinium F1: Replicates for the control and $29^{\circ} \mathrm{C}$ treatments are enclosed by the solid oval; replicates for Day 3 and Day 7 at $33^{\circ} \mathrm{C}$ (dotted oval); and replicates for Day 14 at $33^{\circ} \mathrm{C}$ (dashed oval). 


\section{Discussion}

Integrating the responses of multiple components of the antioxidant network is essential for understanding the specific physiological impact that thermal stress has on different Symbiodinium genotypes. This study revealed that constitutive levels of antioxidants and responses of the network differ between types and that a thermal challenge is not necessarily detected in all parts of the antioxidant system. Activity within different pathways was highly diverse, ranging from solely increased SOD activity in Symbiodinium $\mathrm{E}$ at $33^{\circ} \mathrm{C}$ to up- or down-regulation of all investigated components in Symbiodinium B1. The most tolerant type, Symbiodinium F1, exhibited the largest proportional up-regulation for most antioxidants, whereas the most sensitive type, B1, already exhibited increased glutathione recycling and reduced growth at the sublethal temperature of $29^{\circ} \mathrm{C}$. In all three susceptible types, declining $F_{\mathrm{v}} / F_{\mathrm{m}}$ was associated with an increasing oxidation of the glutathione pool.

\section{Antioxidant profiles are type specific in Symbiodinium}

Removing cellular ROS is a concerted process, involving a complex network of enzymatic and non-enzymatic antioxidants, whose activity is modulated by an equally complex signalling network and affected by the oxidized:reduced ratio of different antioxidants (Mittler et al., 2004, Mullineaux et al., 2006, Mittler et al., 2011). The observed differences in the baseline activities of antioxidants are not surprising, given the high degree of evolutionary radiation in the genus Symbiodinium (LaJeunesse, 2005). Nevertheless, the scale of difference for some of these antioxidants was considerable. The DFA approach shows that using features of their antioxidant network was sufficient to separate and identify the four Symbiodinium genotypes, giving each a unique antioxidant profile. While previous observations that constitutive SOD activities differ between types were confirmed (Lesser, 2011, McGinty et al., 2012), this analysis further illustrated that variability between types is even stronger in parts of the glutathione system, as it mainly drove the cluster separation. The reasons for this are unclear, but SOD provides the primary line of defence against superoxide and therefore affects the amount of ROS that downstream defences have to deal with. In contrast, a number of enzymes can scavenge hydrogen peroxide, providing more 
redundancy. Due to the importance of SOD, activity might be less likely to vary between cell types in comparison to the more downstream mechanisms, which are connected to other systems such as the glutathione system. Temperature responses in the glutathione system also proved to be a key difference between the most tolerant and susceptible types, $\mathrm{F} 1$ and $\mathrm{B} 1$, where relative increases in SOD activity were similar. It has to be acknowledged, however, that given the multiple functions of glutathione in the cell, differences in baseline glutathione pool size and the activity of related enzymes could also be the result of general physiological differences between Symbiodinium types.

\section{Photosynthesis and the SOD-APX pathway}

For plants and algae, stressful temperatures can affect overall cell viability and physiological efficiency, often in conjunction with dramatic decreases in PSII maximum quantum yield of fluorescence and cellular growth. Here, three of the four Symbiodinium genotypes exhibited declining $F_{v} / F_{m}$ values and subsequently died. In Symbiodinium B1, declining $F_{v} / F_{m}$ at $33^{\circ} \mathrm{C}$ was associated with a loss of chlorophyll $a$ within the first week, potentially as a result of photo-oxidative stress (Krieger-Liszkay, 2005). All Symbiodinium genotypes tested here demonstrated increased SOD activity at $33^{\circ} \mathrm{C}$ (though this was not significant for $\mathrm{F} 1$ ). Excessive temperature stress has been linked to Benson-Calvin cycle inhibition, alteration of thylakoid membrane properties with energetic uncoupling, and general D1 degradation and PSIl dysfunction in Symbiodinium (Jones et al., 1998, Takahashi et al., 2004, Tchernov et al., 2004, Hill \& Ralph, 2008). Increased SOD activities in conjunction with declining $F_{v} / F_{m}$ values in $B 1, C 1$, and $E$, may indicate an increased generation of superoxide via the Mehler reaction, potentially as a consequence of a sink limitation for ATP and NADPH in the dark reaction (Asada, 2006). For Symbiodinium F1, increased metabolic turnover with temperature, though not apparent in growth rate, might have contributed to the observed increase in SOD activity. Due to the fact that total SOD activity was determined, it is not possible to assess the relative contribution of superoxide from chloroplastic, mitochondrial and cytosolic sources in these types. Furthermore, since no detergents were used in the extraction buffer, reported global SOD and APX activities will only partially account for the activity of membrane-bound isoforms. Previous studies have, however, shown that increased total SOD activity in 
thermally sensitive Symbiodinium types in vitro and in hospite is generally related to the impairment of photosynthesis (Lesser, 1996, Yakovleva et al., 2004, Richier et al., 2005).

In dinoflagellates, the end product of SOD activity, hydrogen peroxide, can be enzymatically scavenged by APX, catalase peroxidase, peroxiredoxin and potentially Se-containing glutathione peroxidase (GPX) (Osaka et al., 2003, Foyer \& Shigeoka, 2011, Bayer et al., 2012). Interestingly, Symbiodinium E was the only type where APX showed no significant correlation with SOD at $33^{\circ} \mathrm{C}$. Since no obvious hydrogen peroxide-scavenging via APX and the ascorbateglutathione pathway (as GSx did not change either) was observed, elevated concentrations might either have directly contributed to the overall decline in $F_{v} / F_{m}$ or alternative scavenging mechanisms are more prevalent in $E$. Assuming that the site of the most active oxygen metabolism is the chloroplast, alternative pathways such as the 2-Cys peroxiredoxin-dependent water/water cycle may be a potential scavenging route at low hydrogen peroxide concentrations, as peroxiredoxin-encoding genes have been found in a number of Symbiodinium genotypes (Dietz et al., 2002, Bayer et al., 2012). Alternatively, increased hydrogen peroxide-scavenging by cytosolic catalase peroxidase and GPX, or even non-enzymatic detoxification has to be considered. However, APX has a much smaller $K_{m}$ value ( $\mu \mathrm{M}$ range) than, for example a eukaryotic catalase (mM), and is regarded as more responsive to small changes in hydrogen peroxide levels. It is therefore surprising to see no reaction of APX to increased SOD activity in Symbiodinium $\mathrm{E}$.

\section{The glutathione system as an important part of the antioxidant network under thermal stress}

Activity of chloroplastic APX is tightly linked to the glutathione system via the Foyer-Halliwell-Asada cycle, as one mechanism to regenerate oxidized ascorbate (Foyer \& Noctor, 2011). All Symbiodinium genotypes, except E, exhibited considerable increases in their glutathione pool size at the highest temperature, with Symbiodinium B1 temporarily doubling its pool size even at $29^{\circ} \mathrm{C}$. While glutathione pool size in Symbiodinium has rarely been investigated, the values obtained here for Symbiodinium B1, E and F1 fall within the range of coral and anemone holobiont measurements (Kolesar \& Mitchelmore, 2003, Mitchelmore et 
al., 2003, Sunagawa et al., 2008). Interestingly, glutathione levels in Symbiodinium $\mathrm{C} 1$ were an order of magnitude higher than in the other types. While such large pool sizes have also been found for cultures of Symbiodinium A1 and Ax, with even higher pool sizes of up to $2900 \mathrm{nmol} \mathrm{GSx} \mathrm{mg}^{-1}$ protein in A1 (Krämer et al., 2012), the C1 GSx pool is maintained in a highly oxidized state, with an unstressed GSH/GSx value of $50 \%$ compared to $~ 90 \%$ in the other types. Since baseline GR activities and therefore the rate of GSH regeneration are not significantly higher than in Symbiodinium E and F1, it raises the question as to whether the large glutathione pool size in $\mathrm{C} 1$ compensates for the low redox state. On the other hand, low GSH/GSx values might not be unusual for some microalgae, as values of $53 \%$ have also been reported for the planktonic diatom Thallassiosira pseudonana (Rijstenbil, 2002).

Oxidation of the glutathione pool was a common response in all Symbiodinium genotypes when exposed to the highest temperature. Only Symbiodinium F1 was, however, able to stabilize the redox ratio after an initial drop on Day 1. After two weeks, the antioxidant network reached a state with a high turnover of superoxide and Foyer-Halliwell-Asada cycle activity, including the beginning of increased glutathione recycling through GR. This up-regulation of GR after two weeks indicated that the stable glutathione redox state was no longer only met by de novo synthesis of GSH, but also supported by increased glutathione recycling. These processes were also apparent in the most susceptible type, Symbiodinium B1, where they started at sublethal temperatures that did not affect photosynthetic efficiency. While the stress response of Symbiodinium B1 at $33^{\circ} \mathrm{C}$ was similar to that in $\mathrm{F} 1$, but more immediate, the glutathione system switched from a reduced state at $29^{\circ} \mathrm{C}$ to a highly oxidized state at $33^{\circ} \mathrm{C}$ due to the collapse of the recycling mechanism. This led to a situation where an increased activity of the ascorbate-glutathione pathway under thermal stress caused a progressive oxidation of the glutathione pool, a potential pro-apoptotic signal. Moreover, increased GST activity at $33^{\circ} \mathrm{C}$ consumed GSH from the pool, exacerbating the glutathione redox imbalance. Plant GSTs represent a large family of proteins, and remove peroxides and electrophilic compounds that may potentially attack DNA, RNA and proteins (Noctor et al., 2002). Studies measuring GST in Symbiodinium when in hospite have produced mixed results in 
response to anthropogenic contaminants (Downs et al., 2005, Ramos \& García, 2007), but this is the first study to show a temperature response at the proteomic level.

Variability in the temporal response and the importance of other ROS-removal pathways has to be considered, when interpreting the responses of Symbiodinium E and C1. In Symbiodinium E, oxidative stress was only apparent with respect to SOD activity, with no significant upregulation in the ascorbate- or glutathione-related enzymatic systems, though the glutathione redox state continued to decline, effectively lowering the amount of available GSH for metabolism at this temperature. On the other hand, a significant upregulation of the SOD-APX pathway and the glutathione content in Symbiodinium $\mathrm{C} 1$ at $33^{\circ} \mathrm{C}$ was only observed on the last day, when $F_{v} / F_{m}$-values had already declined considerably. This discrepancy indicates that alternative protective measures, including photoprotective measures upstream of antioxidant defences, or nonenzymatical scavenging might be more relevant for determining the thermal threshold in this type.

\section{Antioxidant plasticity as a key for acclimation}

All Symbiodinium types investigated here expressed considerable differences in their antioxidant baseline activities. Since the most tolerant type, F1, also had the highest activities for the SOD-APX pathway, it seems tempting to link its thermal tolerance to a fundamentally higher scavenging activity in this part of the network. However, one has to consider that this does not necessarily translate to an actual advantage, as it has been shown that steady state fluxes of ROS in some Symbiodinium genotypes might simply be higher (McGinty et al., 2012). Irrespective of the type-specific differences found here, the ability to activate and upregulate the activity of these enzymes under stress is the fundamental mechanism that allows organisms to cope with ROS (Finkel \& Holbrook, 2000). In terms of proportional upregulation relative to baseline activity, Symbiodinium F1 was superior to all types. This greater capacity to enhance components of its antioxidative defences probably contributed to the maintenance of cellular viability in a thermal environment that was lethal to all other types. In this context, this study agrees with previous publications on photophysiological responses, which have highlighted that the capacity to upregulate protective mechanisms 
tends to be a key element of thermally tolerant Symbiodinium types (Robison \& Warner, 2006, Takahashi et al., 2009, Ragni et al., 2010). This physiological plasticity is also observed in the adjustments of the glutathione system in Symbiodinium B1, however only up to $29^{\circ} \mathrm{C}$. Both cases illustrate that the ability to maintain cellular homeostasis under thermal stress in Symbiodinium relies on the plasticity of the antioxidant network and its role in defining the "threshold at which a cell or tissue makes the transition from successful acclimation/resistance to oxidative stress-induced cell death" (Mullineaux \& Baker, 2010).

The data presented here illustrate the complex responses of symbiotic dinoflagellates in culture. The results, however, have to be viewed as an approximation of the processes in the intact symbiosis and emphasize the mechanistic viewpoint rather than predicting in situ processes. From an ecological point of view, this study demonstrates that elevated seawater temperatures, while lethal for most Symbiodinium genotypes beyond $30^{\circ} \mathrm{C}$, may already have a profound sublethal impact, by altering growth rates and energy investment. Such impacts may influence population dynamics in mixed environmental Symbiodinium pools and possibly in the intact symbiosis.

In summary, I have shown that the differential thermal susceptibility of Symbiodinium genotypes in culture can be related to the activity of their antioxidant network. Baseline activities, scale of upregulation in the SOD-APX pathway, and mechanisms to maintain redox homeostasis in the glutathione system, all contributed to overall cell viability and defined the type-specific response to a thermal challenge. Significant network shifts in antioxidant activity were evident in two Symbiodinium types and illustrate the importance of antioxidant plasticity in maintaining cellular viability. This study provides an important link between Symbiodinium diversity and stress physiology, and delivers insights into the biochemical responses that may determine the fate of different Symbiodinium types with varying thermal tolerances. 
- 52 - 


\section{Transcriptomic and proteomic characterization of enzymatic antioxidants in the dinoflagellate genus Symbiodinium}

\section{Introduction}

The photosynthetic dinoflagellate Symbiodinium sp. is a significant endosymbiont of a wide range of marine invertebrates (reviewed in Stat et al., 2006). It is also a major contributor of the ecological success of zooxanthellate scleractinian corals in oligotrophic shallow tropical seas (Muscatine \& Hand, 1958, Muscatine \& Porter, 1977). Phylogenetic diversity within the genus Symbiodinium, based on the internal transcribed spacer region (ITS2), is high, with currently nine major clades (A-I) and hundreds of sub-clades ('types') recognized (Coffroth \& Santos, 2005, Pochon \& Gates, 2010). The mutualistic association of these dinoflagellates with corals has gained much attention through the phenomenon of coral bleaching, where a decline in symbiont density and/or photosynthetic pigment content in response to environmental stress results in the visible paling of the coral (Glynn, 1993, Glynn, 1996). Coral bleaching can occur on both local and mass scales and culminate in large scale coral mortality as a result of climate change-induced warming of the oceans. Hence, coral bleaching represents a major threat to the functioning of coral reef ecosystems (Hoegh-Guldberg, 1999, Hughes et al., 2003).

Variability in bleaching severity between different coral-Symbiodinium associations under similar temperatures has highlighted the role of Symbiodinium diversity in determining the performance of the coral holobiont, i.e., the combination of symbiont and coral (Rowan, 2004a, Berkelmans \& van Oppen, 2006, Stat et al., 2008b, Fisher et al., 2012). While the physiological mechanisms of bleaching are not yet fully understood, the generation of reactive oxygen species (ROS) in the symbiont under stress is thought to play an important role in the cellular bleaching cascade (reviewed in Weis, 2008, Lesser, 2011), and differences in ROS generation or antioxidant defences between Symbiodinium types could contribute to the variable bleaching susceptibilities of corals. 
Reactive oxygen species (ROS) such as superoxide or hydrogen peroxide occur as side-products of oxygenic photosynthesis and respiration (Halliwell \& Gutteridge, 2007), and antioxidants have an important role in maintaining redox homeostasis and preventing any associated oxidative damage. Enzymatic scavenging of ROS works via a number of pathways and one of the first lines of defence is the scavenging of superoxide by superoxide dismutase (SOD). SOD is expressed as a number of metalloproteins with different isoforms of which some are plastid-specific (e.g., mitochondrial MnSOD or chloroplastic FeSOD; Alscher et al., 2002). Different forms of cellular peroxidases and catalases act as downstream defences that deal with the end product of SOD activity, hydrogen peroxide $\left(\mathrm{H}_{2} \mathrm{O}_{2}\right)$. The activity of these peroxidases is also dependent on the availability of low molecular weight antioxidants, such as ascorbate and glutathione, which act as important cofactors and are essential to the hydrogen peroxide metabolism of photosynthetic organisms (reviewed in Foyer \& Noctor, 2011).

Differential activity of antioxidants in different Symbiodinium types under thermal stress has been shown at the proteomic level, both for algal cultures and intact corals (Chapter 2; Lesser, 1997, Yakovleva et al., 2009). SOD, ascorbate peroxidase (APX) and the bacterially-derived enzyme catalase peroxidase (KatG) have all been shown to be involved in the response to light and temperature stress (Lesser, 1996, McGinty et al., 2012). The application of high-throughput sequencing technologies to the coral-dinoflagellate symbiosis has provided a large body of transcriptomic data for a number of coral species (Kortschak et al., 2003, Forêt et al., 2007, Miller et al., 2007, deSalvo et al., 2008, Schwarz et al., 2008, Shinzato et al., 2011), but only a few Symbiodinium types (Leggat et al., 2007, Bayer et al., 2012). While changes in host expression levels under thermal stress for genes related to oxidative stress such as ferritin, heat shock proteins, glutathione S-transferase, MnSOD or catalase have been shown (Edge et al., 2008, Schwarz et al., 2008, Császár et al., 2009, Rodriguez-Lanetty et al., 2009, Polato et al., 2010), only a few studies have investigated the transcriptomic response of a major enzymatic antioxidant in the symbiont to date (Mayfield et al., 2011, Putnam et al., 2012a). Considering their pivotal role in coral health and evolutionary fitness under environmental stress, it is essential to extend the 
transcriptomic toolkit to allow a comparison of antioxidant expression patterns in a variety of Symbiodinium types, incorporating cladal and sub-cladal diversity.

The focus of this study was therefore to generate gene transcripts for enzymatic antioxidants from a number of Symbiodinium types, in order to investigate the degree of sequence variation at the amino acid level and assess their phylogenetic relationships. Furthermore, antioxidant gene expression and activity at the proteomic level was investigated in Symbiodinium ITS2 type B1 under thermal stress.

\section{Material and Methods}

\section{Sequence characterization and phylogenetic analysis of antioxidant genes}

Symbiodinium types, RNA isolation, and cDNA generation

RNA from $10 \mathrm{~mL}$ of concentrated batch culture (2000 $\times \mathrm{g}, 5 \mathrm{~min})$ of the Symbiodinium types A1 (culture ID CCMP2467), B1 (Ap1), C1 (CCMP2466), C3 (Mp), E (CCMP421) and F1 (Mv) was extracted with a bead mill (50 Hz, 5 min, $4^{\circ} \mathrm{C}$ ), using the Purelink ${ }^{\circledR}$ RNA Mini Kit (Life technologies), and reverse transcribed into cDNA (QuantiTect Reverse Transcription Kit, Qiagen) according to the manufacturer's instructions. In addition, cDNA from Symbiodinium C15 (isolated from Montipora digitata, Heron Island) was provided by Dr. Paul Fisher (Davy Lab, Victoria University of Wellington, New Zealand). All Symbiodinium identities are based on ITS2 analysis, performed as published previously (Logan et al., 2010).

\section{Primer design and amplification strategy}

Amino acid sequences for the genes of interest - MnSOD, FeSOD, APX and KatG - from closely related taxa were obtained from GenBank (Benson et al., 2010), Uniprot (Consortium, 2013) and Peroxibase (Fawal et al., 2013), and blasted against Symbiodinium C1 and C3 EST databases (Leggat Lab, James Cook University, Townsville, Australia). Matching contigs were aligned to generate the longest possible consensus sequence for the coding region of interest. Random primers, based mainly on the retrieved longer Symbiodinium C3 sequence for each enzyme, were generated using the Primer3 (Untergasser et al., 2012) plug-in in Geneious® 6.1.7 (Biomatters Ltd., Auckland, New Zealand). 
Symbiodinium C3 sequences for genes of interest were also used to search for contigs in other available Symbiodinium EST libraries, such as A1 (CCMP2467 library, Voolstra Lab, King Abdullah University of Science and Technology, Saudi Arabia) and B1 (mf105 library, Medina Lab, Pennsylvania State University, USA), and respective type-specific primers designed. In addition to the random and type-specific primer approach, a splice leader primer (SL-primer; 5'CCGTAGCCATTTTGGCTCAAG-3'; Zhang \& Lin, 2009) was tested in conjunction with working reverse primers to amplify the $\mathrm{N}$-terminal coding region for each enzyme.

Primers were tested using a general PCR amplification profile, consisting of an initial denaturation of $3 \mathrm{~min}$ at $95^{\circ} \mathrm{C}$, followed by 35 cycles of $30 \mathrm{sec}$ at $95^{\circ} \mathrm{C}, 30$ sec at $54^{\circ} \mathrm{C}$ and $1 \mathrm{~min}$ at $72^{\circ} \mathrm{C}$ with a final elongation of $7 \mathrm{~min}$ at $72^{\circ} \mathrm{C}$, using the MyTaq $^{\text {TM }}$ Red Mix (BIOLINE). PCR amplicons were visualized via gel electrophoresis (1.5-3.5\% [w/v] agarose, using molecular ladders Hyperladder II or $\mathrm{V}$ [BIOLINE] for estimation of amplicon size). Successful amplifications were purified using ExoSAP-IT (Global Science) and sequenced by Macrogen Inc. (Seoul, South Korea). In case non-specific amplifications occurred, annealing temperatures were increased up to $60^{\circ} \mathrm{C}$ to increase PCR specificity and/or bands were manually excised and purified (Zymoclean ${ }^{\mathrm{TM}}$ Gel DNA Recovery Kit).

Identification, alignment and annotation of coding sequences

Partial sequences for each gene of interest and Symbiodinium type were aligned using Clustal W (Thompson et al., 1997) and the consensus sequence blasted (Altschul et al., 1990) against GenBank. Obtained sequences for types A1 (CCMP2467), B1 (Ap1), C1 (CCMP2466) and C3 (Mp) were also validated against their respective EST library entries. Sequences were analysed for the presence of open reading frames (ORF) using ATGpr software (Salamov et al., 1998) and translated accordingly. Amino acid sequences were analysed using EMBL-EBI InterProScan (Zdobnov \& Apweiler, 2001) for general features, and TargetP 1.1 (Emanuelsson et al., 2000) for the presence of signal peptides and their cleavage sites. If full length sequences were obtained, 3-D models, based on the mature amino acid sequence (without $\mathrm{N}$-terminal signal) were developed using i-tasser (Zhang, 2008, Roy et al., 2010). Alignment of 3-D models and visual highlighting of specific features was done in Geneious ${ }^{\circledR}$ 6.1.7. 


\section{Phylogenetic analyses}

Sequences were aligned using Clustal $\mathrm{W}$ and trimmed to equal lengths if necessary. Appropriate phylogenetic models for protein evolution were determined using ProtTest (Abascal et al., 2005) and tree topology determined using maximum likelihood analysis with a bootstrap analysis (100 replicates) to infer topology robustness (Felsenstein, 1985, Hillis \& Bull, 1993). Phylogenetic trees were generated using the PhyML (Guindon \& Gascuel, 2003) plug-in in Geneious ${ }^{\circledR}$ 6.1.7.

\section{Gene expression experiment}

\section{Experimental setup}

Six batch cultures of Symbiodinium B1 (culture ID Ap1) were grown in $2 \mathrm{~L}$ roundbottom flasks, containing silica-free sterile $\mathrm{f} / 2$-medium $(\mathrm{pH}$ 8.1), based on synthetic seawater (salinity 34; Instant Ocean sea salt, Spectrum Brands Inc., USA). Cultures were aerated with filter-sterilized air $(0.22 \mu \mathrm{m})$ and grown on a constant 12-h/12-h light/dark cycle under an irradiance of $40-50 \mu \mathrm{mol}$ quanta $\mathrm{m}^{-2}$ $\mathrm{s}^{-1}$ (cool white fluorescent tubes, Philips $36 \mathrm{~W} / 840$ ) at $25^{\circ} \mathrm{C}$ in temperaturecontrolled tanks. After 12 days of acclimation, these six batch cultures served as inocula for 6 control and 6 treatment flasks that were allocated to two tanks, respectively. Initial densities were set to approximately $1.5 \times 10^{5}$ cells $\mathrm{mL}^{-1}$. After sampling on Day 0 , the temperature was increased to $33^{\circ} \mathrm{C}\left(1^{\circ} \mathrm{C} \mathrm{h}^{-1}\right)$ in the treatment tank during the dark phase, while the control treatment was maintained at $25^{\circ} \mathrm{C}$. Based on the response in previous experiments (Chapter 2), all cultures were sampled in the middle of the light phase on Day 0, Day 1 and Day 3, by taking a total volume of $350 \mathrm{~mL}(7 \times 50 \mathrm{~mL}$ aliquots $)$ each day. Cells were pelleted $(2000 \times \mathrm{g}, 5 \mathrm{~min})$, flash frozen in liquid nitrogen and stored at $-80^{\circ} \mathrm{C}$. In addition, $5-10 \mathrm{~mL}$ aliquots were taken for cell density determination (fixing them in $4 \%$ formalin) and PAM measurements (see below for details).

\section{Viability}

Growth, maximum quantum yield of PSII $\left(\mathrm{F}_{\mathrm{v}} / \mathrm{F}_{\mathrm{m}}\right)$ and chlorophyll a $(\mathrm{Chl}$ a) content per cell were monitored as proxies for cell viability. After $20 \mathrm{~min}$ of dark adaptation, $F_{\mathrm{v}} / F_{\mathrm{m}}$ of $2 \mathrm{~mL}$ of live culture was measured using a Water-PAM chlorophyll fluorometer (Heinz Walz GmbH, Effeltrich, Germany). Haemocytometer counts $(N=6)$ were used to calculate the specific growth rate 
for each replicate via a linear regression fit of log-transformed cell densities from all three sampling days (Guillard, 1973). Chl a of one $50 \mathrm{~mL}$ pellet was extracted over $48 \mathrm{~h}$ in $1-2 \mathrm{~mL} \mathrm{~N}, \mathrm{~N}$-dimethylformamide at $4^{\circ} \mathrm{C}$ in the dark. After centrifugation $\left(3 \mathrm{~min}, 3900 \times \mathrm{g}, 4^{\circ} \mathrm{C}\right.$ ), $3 \times 200 \mu \mathrm{L}$ of the supernatant (technical triplicate) were measured at $646.8 \mathrm{~nm}, 663.8 \mathrm{~nm}$ and $750 \mathrm{~nm}$ in 96-well plates (UVStar, Greiner Bio-One GmbH, Frickenhausen, Germany). Chl a concentrations were determined after optical path length correction $(0.555 \mathrm{~cm})$ (cf. Chapter 2; Porra et al., 1989).

\section{Molecular analysis}

Three frozen pellets per replicate and time-point were pooled and lysed in cold buffer (50 mM KH $\mathrm{PO}_{4} / \mathrm{K}_{2} \mathrm{HPO}_{4}, 0.1 \mathrm{mM}$ EDTA, 10\% [v/v] glycerol) using ca. 200 mg glass beads (710-1180 um; Sigma-Aldrich) in a bead mill $\left(50 \mathrm{~Hz}, 3 \mathrm{~min}, 4^{\circ} \mathrm{C}\right.$; Qiagen tissue lyser, Qiagen N.V., Hilden, Germany). All lysates were centrifuged $\left(16000 \times \mathrm{g}, 5 \mathrm{~min}, 4^{\circ} \mathrm{C}\right)$ and supernatants aliquoted and frozen at $-80^{\circ} \mathrm{C}$ until further analysis. Total aqueous soluble protein content was measured with BSA as a protein standard (Zor \& Selinger, 1996). Assays for SOD and APX were performed as outlined in Chapter 2. Catalase peroxidase activity was determined spectrophotometrically using $100 \mu \mathrm{L}$ lysate in a total reaction volume of $700 \mu \mathrm{L}$, containing potassium phosphate buffer $(50 \mathrm{mM}, \mathrm{pH} 7.0)$, EDTA $(0.1 \mathrm{mM})$ and hydrogen peroxide (14 mM). The reaction was monitored for $3 \mathrm{~min}$ at $240 \mathrm{~nm}$ and $25^{\circ} \mathrm{C}$ in quartz cuvettes (UV-Vis Spectrophotometer UV-2550, Shimadzu Corp., Kyoto, Japan) and activities calculated using an extinction coefficient of $43.6 \mathrm{M}^{-1}$ $\mathrm{cm}^{-1}$ (Beers \& Sizer, 1952). All enzyme activities were normalized per cell and expressed as specific activity $\left(\mathrm{U}^{\mathrm{cell}}{ }^{-1}\right)$, where one unit catalyses one $\mu \mathrm{mol}$ substrate $\min ^{-1}$ cell $^{-1}$.

RNA of three pooled pellets per replicate and time-point was extracted with a bead mill $\left(50 \mathrm{~Hz}, 5 \mathrm{~min}, 4^{\circ} \mathrm{C}\right.$ ), using the Purelink ${ }^{\circledR}$ RNA Mini Kit (Life technologies) and reverse transcribed into cDNA (QuantiTect Reverse Transcription Kit, Qiagen) according to the manufacturer's instructions. 
Primer design and amplification of housekeeping genes and genes of interest for Symbiodinium $B 1$

The housekeeping genes (HKG) B-tubulin (Tub), S-adenosyl methionine synthetase (SAM), calmodulin (Cal), and cytochrome oxidase subunit 1 (Cox) were chosen based on a previous study (Rosic et al., 2011b). FeSOD, MnSOD, $A P X$ and KatG were chosen as genes of interest. Partial sequences for HKG were obtained by either using the available EST library for Symbiodinium B1 (mf105 library, Medina Lab, Pennsylvania State University, USA) or testing the SL-primer in combination with reverse HKG primers previously used for Symbiodinium C3 (Zhang \& Lin, 2009, Rosic et al., 2011b). All primers were designed with Primer3 (Untergasser et al., 2012) as a plug-in in Geneious ${ }^{\circledR} 6.1 .7$ (Biomatters Ltd), with melting temperatures of $60^{\circ} \mathrm{C}$, and tested as outlined above. Sequences were identified by means of BLAST (Altschul et al., 1990) against GenBank, and qPCR primers designed.

Quantitative polymerase chain reaction ( $q P C R$ )

Designed qPCR primers for HKG and target genes were tested on a pooled cDNA sample, constructed from RNA extracted from all six Symbiodinium B1 replicates in the control treatment on Day 0. Equimolar primer pair concentrations were optimized in the range 200-1000 nM, and efficiency assessed using a template dilution series (Table 3.1). Specificity and product size were assessed via melt curve analysis and gel electrophoresis. qPCRs were performed with $2 \mu \mathrm{L}$ 1:50 diluted template in $20 \mu \mathrm{L}$ reaction volume, using a Power SYBR $B$ Green Master Mix (Life Technologies). Thermocycling profiles were run on a StepOne ${ }^{\mathrm{TM}}$ Real-Time PCR machine (Applied Biosystems, Foster City, CA, USA), consisting of an initial incubation for $10 \mathrm{~min}$ at $95^{\circ} \mathrm{C}$, followed by 40 cycles of $15 \mathrm{sec}$ at $95^{\circ} \mathrm{C}$ and $1 \mathrm{~min}$ at $60^{\circ} \mathrm{C}$. The run was concluded with a melt curve from $60^{\circ} \mathrm{C}$ to $95^{\circ} \mathrm{C}$ to assess primer specificity. Despite testing a number of FeSOD qPCR primer pairs, it was not possible to consistently amplify this enzyme. 
Table 3.1. Primer characteristics for gene expression of Symbiodinium B1.

Primer sequence, amplicon size, equimolar concentration and efficiency for potential HKGs and target genes (APX, KatG, MnSOD) used in qPCR of Symbiodinium B1.

\begin{tabular}{|c|c|c|c|c|c|}
\hline Gene & $\begin{array}{l}\text { Forward primer } \\
\qquad\left(5^{\prime}-3^{\prime}\right)\end{array}$ & $\begin{array}{c}\text { Reverse primer } \\
\qquad\left(5^{\prime}-3^{\prime}\right)\end{array}$ & $\begin{array}{l}\frac{0}{N} \\
\frac{0}{0} \\
\frac{0}{0} \\
\frac{0}{0} \\
\frac{0}{2} \\
\frac{\varepsilon}{4}\end{array}$ & 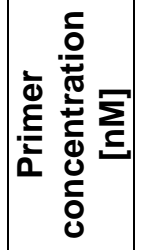 & 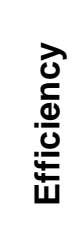 \\
\hline $\mathrm{Cal}$ & TGATGGCGCGCAAGATGAAGG & TGCCATCGCGATCGAAAACCTTG & 78 & 750 & $98 \%$ \\
\hline Cox & TСTGTCTTССTСTСАСАТСTCT & CCACTGCACCATTTCCAAGA & 82 & 225 & $97 \%$ \\
\hline SAM & GACCAAGAACGGCATCAAGT & TGCTGCTCATGGATGCATAC & 74 & 200 & $95 \%$ \\
\hline Tub & CCAGCTTTGGCATTCCCTTG & TGGTTCCACCACTGTGTCAG & 148 & 750 & $94 \%$ \\
\hline$A P X$ & CAATGTGGCACTCATGCTGG & TAAGCTTCTCAAGGTCCGCC & 107 & 500 & $102 \%$ \\
\hline KatG & TCTTCTTGGCCAAGTGAAGC & TTTGATGGCAGTGGTTCCTG & 85 & 500 & $96 \%$ \\
\hline$M n S O D$ & CAACCCCAAACCAGGACAAT & CACATCCCACCAAGCTTTGA & 146 & 1000 & $96 \%$ \\
\hline
\end{tabular}

Statistical analysis

All physiological variables were analysed for temperature effects using repeated measures analysis of variance (rmANOVA). Cell densities were log transformed and $F_{v} / F_{m}$ values were arcsine square root transformed. Datasets for each variable were tested for sphericity with Mauchly's sphericity test. Results of Pillai's trace test or epsilon-adjusted univariate F-test (Greenhouse-Geisser; G-G) at a confidence level of 0.05 are reported. Reported post hoc contrasts of means between Day 0 and day of observation were adjusted for multiple comparisons, using the Bonferroni correction. Stable HKG were identified by comparing HKG expression at $25^{\circ} \mathrm{C}$ and $33^{\circ} \mathrm{C}$ using the software Normfinder (Andersen et al., 2004). $C_{t}$-values for all samples were used to calculate relative gene expression of target genes and unsuitable HKG, using REST 2009 with 10000 iterations (Pfaffl et al., 2002).

\section{Results}

\section{Molecular characterization of antioxidant genes in the genus Symbiodinium}

\section{Manganese superoxide dismutase}

Obtained cDNA sequences were $\sim 800 \mathrm{bp}$ in length and covered the whole reading frame of 266 amino acids (aa) for types B1, C1, C3 and C15. Blasting these sequences on available Symbiodinium EST libraries revealed identical or 
similar MnSOD contigs for Symbiodinium A1 (Locus 9072, Locus 7642), B1 (mf105_rep_c3255 [identical to Ap1], mf105_c9010, mf105_c13368), D (GAFP01017905) and F1 (KC937118). All sequences were identified as dimeric MnSOD, and signal peptides, containing transmembrane domains were found in all sequences, designating them as part of the mitochondrial or secretory pathway (Figure 3.1). However, prediction strength of their subcellular location given by TargetP1.1 ("plant" setting) was low (reliability class 4-5). Dinoflagellate splice leaders were identified in $\mathrm{F} 1$ and $\mathrm{C} 15,57$ and 45 bp upstream of the start codon, respectively. A similar isoform of MnSOD was found for B1, D and F1, where the final stop codon was replaced by a codon for aspartic acid. The subsequent extension of 31-33 aa contained two short a-helices. Thus, the monomeric molecular weight of 25.4-25.5 kDa (all C types and B1 (Ap1)) increased slightly to 28.7-29.3 in B1 (mf105), D, and F1 for the mature (without signal peptide) protein. The extension increased the protein surface, altered the spatial orientation of the $\mathrm{N}$-terminus (Figure 3.2A), and lowered the predicted isoelectric point in Symbiodinium B1 (6.52 vs. 5.24). Sequence identity of the mature protein was highest between members of clade $\mathrm{C}$, but also high between D and $\mathrm{C} 15$ (>97\% sequence identity; $\leq 7$ substitutions) (Appendix Table 7.1 ). Overall differences ranged from 9-17 substitutions for B1 (mf105 and Ap1) against the $C$ and $D$ clades ( 93-96\% identity). The predicted monomer structure of the short MnSOD isoform between Symbiodinium B1 and a clade $\mathrm{C}$ member was nearly identical (Figure 3.2B). The highest degree of substitutions against all other clades was seen in $\mathrm{F} 1$, with 20-30 substitutions translating to a sequence identity of $87-93 \%$. All types grouped into a single cluster, phylogenetically separated from another cluster that contained sequences of Bacillariophyceae, Phaeophyceae, Cryptophyceae and the heterotrophic dinoflagellate Noctiluca scintillans. The pelagophyte Aureococcus anophagefferens was found to take an intermediate position between these two clusters (Figure 3.3). 


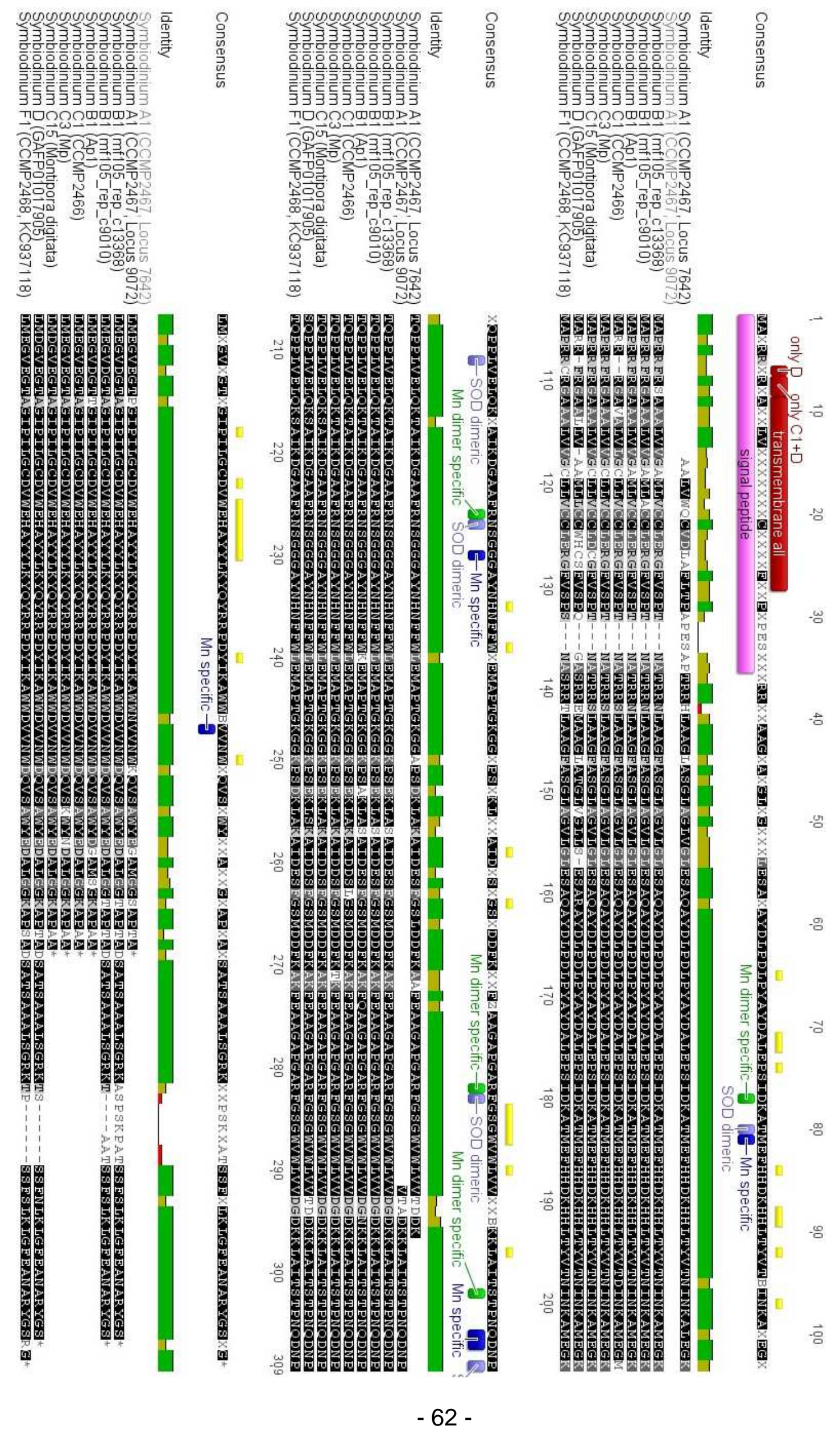


[previous page]

Figure 3.1. Protein alignment of Symbiodinium manganese superoxide dismutase sequences.

Alignment of manganese superoxide dismutase (MnSOD) protein sequences derived from different Symbiodinium types with annotation of functionally important amino acid residues and the $\mathrm{N}$-terminal signal peptide (magenta) with transmembrane domain (red). Identification as a dimeric MnSOD isoform was based on specific residues highlighted on the consensus sequence. Conserved SOD residues (yellow), SOD dimer-specific residues $\mathrm{Thr}^{80}$, $\mathrm{Leu}^{108}, \mathrm{Asn}^{124}$, $\mathrm{Phe}^{180}$, and $\mathrm{Pro}^{206}$ (purple), MnSOD specific residues $\mathrm{Met}^{81}, \mathrm{Gly}^{127}$, Gln ${ }^{203}, \mathrm{Asp}^{204}$ (blue) and manganese dimer-specific residues Asp ${ }^{77}, \mathrm{Arg}^{123}$, $\mathrm{Arg}^{179}$, and $\mathrm{Ser}^{199}$ (green). Annotations were deducted from alignments with characterized sequences from Wintjens et al. (2008). Sequence similarities are $100 \%$ (black), $80-100 \%$ (dark grey), $60-80 \%$ (light grey), and less than 60\% (white), based on the Blosum62 score matrix with a threshold of 1 . Culture ID, host and/or accession numbers given in brackets.
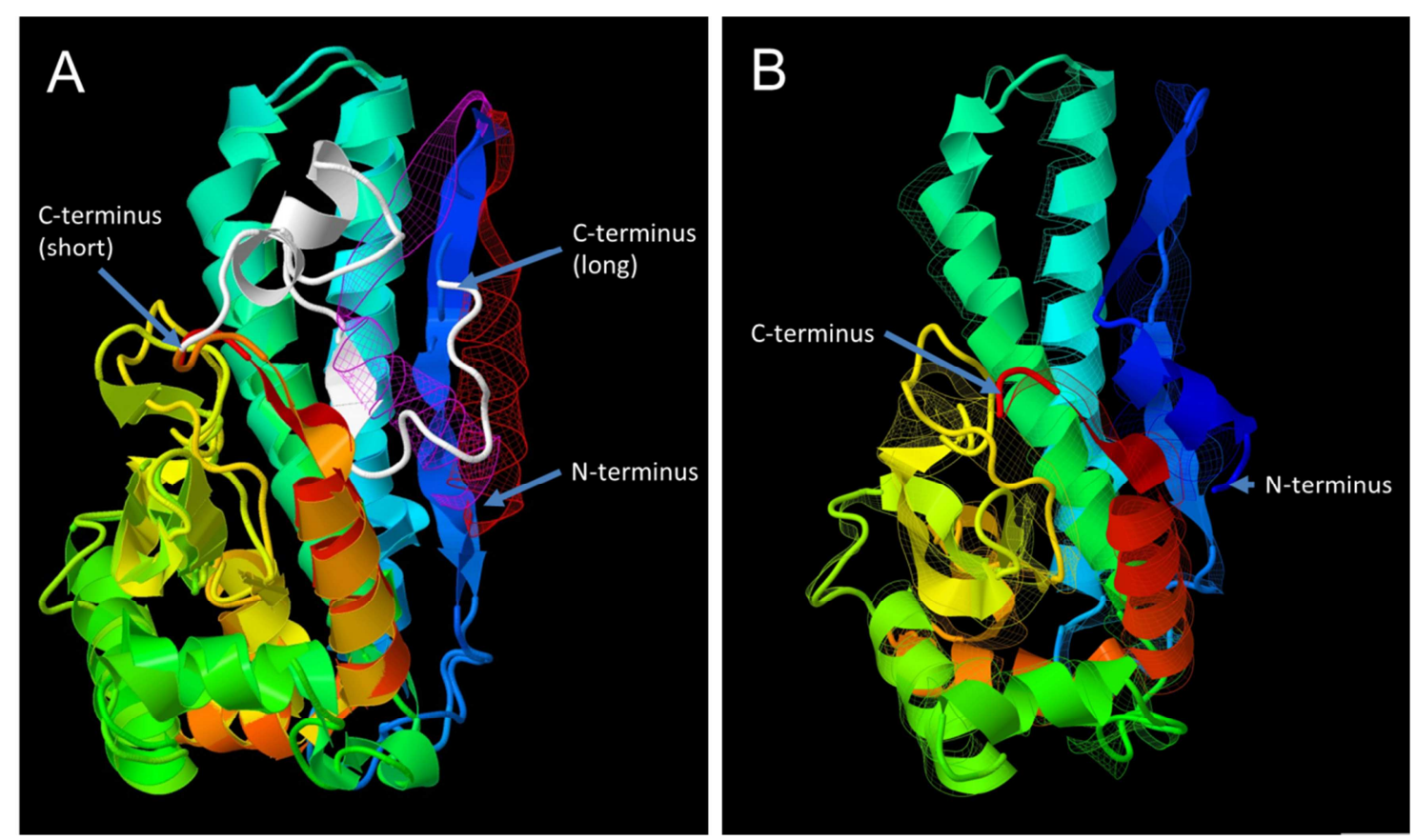

Figure 3.2. Predicted monomer structures of Symbiodinium manganese superoxide dismutase isoforms.

(A) Superimposition of predicted monomer structure from two manganese superoxide dismutase (MnSOD) isoforms from Symbiodinium B1 (Ap1, C-score $=0.30$ and mf105_rep_c9010, C score $=-0.74$ ) (cf. Figure 3.1). The structural extension in the longer isoform from B1 (mf105_rep_c9010) is highlighted in white, indicating the new location of the C-terminus (long). Spatial orientation of the $\mathrm{N}$-terminus is highlighted as mesh ribbon in the short (magenta) and long (red) isoforms. (B) Superimposition of the predicted monomer structure for the shorter MnSOD form from Symbiodinium B1 (Ap1, solid, C-score $=0.30$ ) and Symbiodinium C1 (CCMP2466, mesh ribbon, C-score $=0.32$ ). Colour temperature indicates direction from the $\mathrm{N}$ - to the $\mathrm{C}$-terminus (blue to orange). 


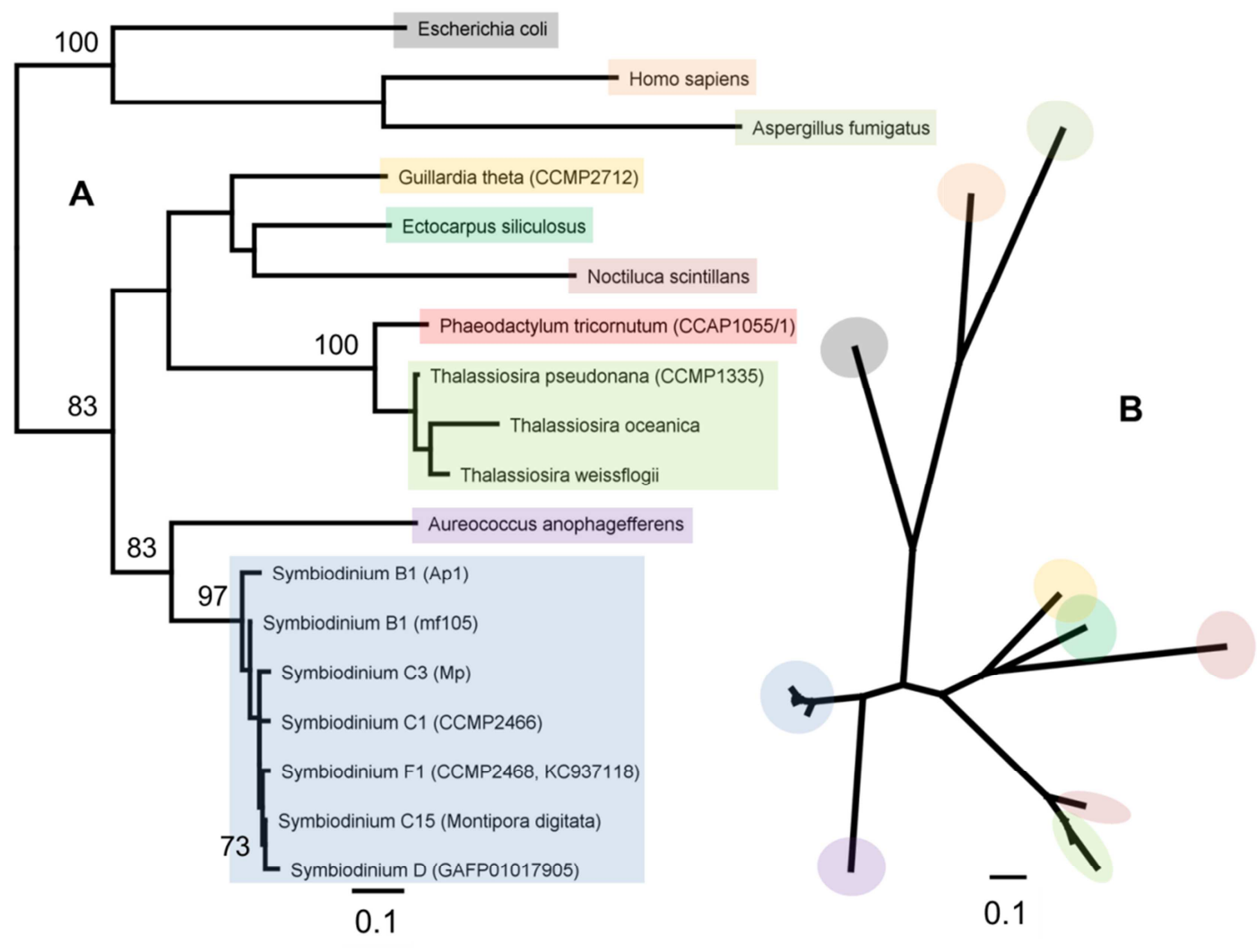

Figure 3.3. Protein phylogeny of manganese superoxide dismutase.

Manganese superoxide dismutase (MnSOD) phylogeny of Symbiodinium sequences and other taxa inferred from maximum likelihood analysis as rooted (A) and unrooted trees (B). Scale bars indicate the number of amino acid substitutions and bootstrap percentages $>70 \%$ are indicated on nodes. Location of clusters in the unrooted tree is indicated by colour. Culture ID, host and/or accession numbers given in brackets.

\section{Iron superoxide dismutase}

Obtaining FeSOD sequences was less successful than for MnSOD, and only partial sequences for Symbiodinium B1, E and F1 were determined. Attempts to measure expression of FeSOD in B1 via qPCR also indicated comparatively low transcript abundance and problems with primer specificity. A previously published Symbiodinium A (PF-2005, AY916504) sequence determined the protein length of the complete reading frame with 201 aa $(21.9 \mathrm{kDa})$ and a predicted isoelectric point of 5.18. No signal peptide was found. Partial sequences obtained here covered $66-74 \%$ of this total protein length (Figure 3.4). Alignments indicated a high degree of residue conservation ( $>94 \%$ ) between the four types A, B1, E and $\mathrm{F} 1$, whereas similarity at the nucleotide level relative to clade $A$ was in the range of $81-86 \%$ (Appendix Table 7.1). Blasting FeSOD protein sequences against other Symbiodinium EST libraries yielded only MnSOD sequences as hits. 


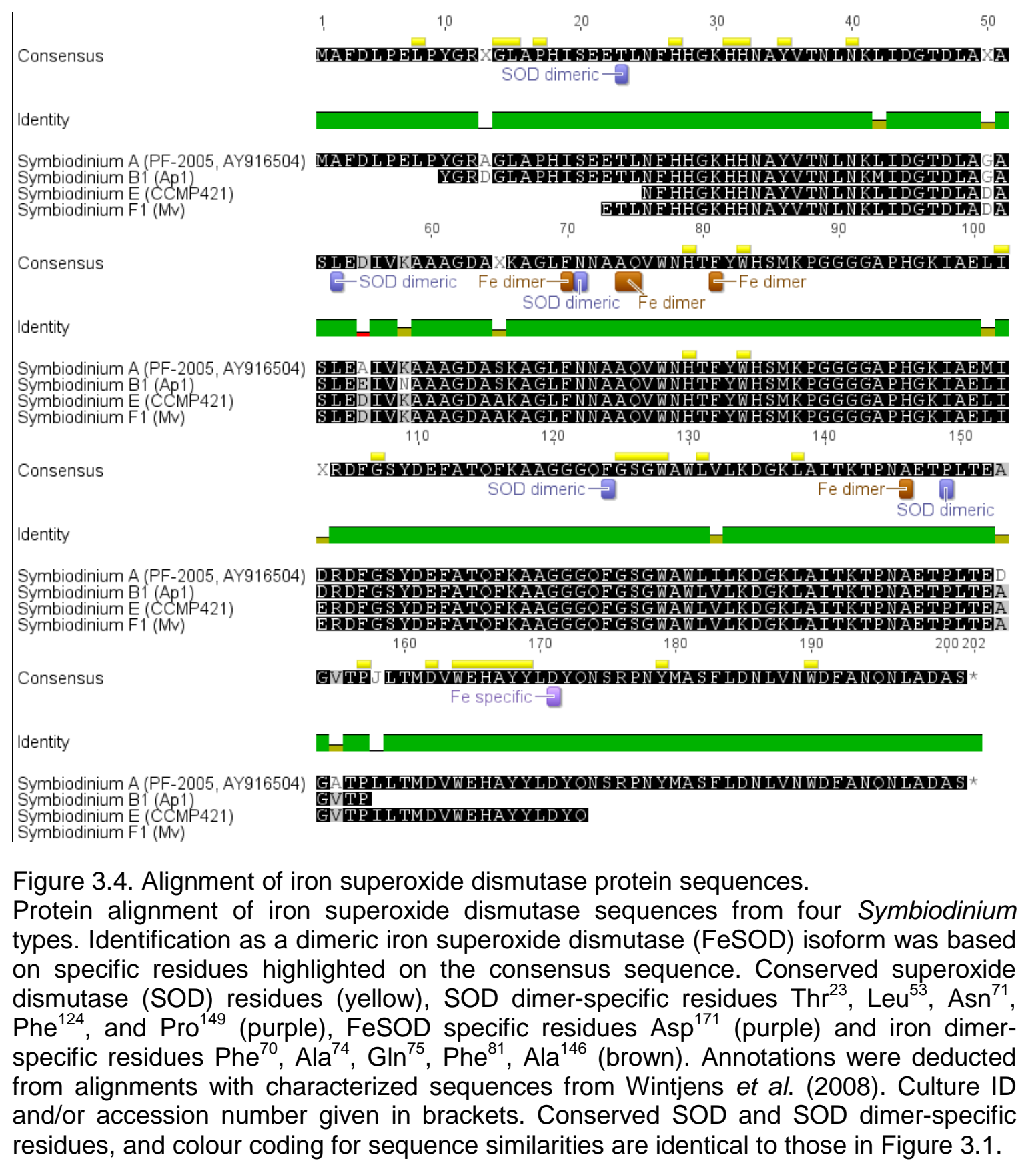

\section{Ascorbate peroxidase}

Using the SL primer as the forward primer worked only for the APX transcript of Symbiodinium F1. While analysis of the ORF frames indicated that the overall protein is at least 390-400 aa long, multiple start codons can be found within the reading frame. The obtained APX transcripts were identical to EST library entries for A1 (Locus 1984), B1 (mf105 rep c1624) and F1 (KC944346, KC951049), and an additional APX transcript was found for Symbiodinium A1 (Locus 9887) in the CCMP2467 library. This isoform more closely matched the APX consensus of all 
other Symbiodinium types and was included in the alignment (Figure 3.5). No signal peptides were found in the $\mathrm{N}$-terminal domain of types $\mathrm{C} 3$ and $\mathrm{F} 1$. Overall sequence identity between clades decreased in the order $(C>B>F>A)$. Variability between types was most prominent between residues 248 and 259. Sequence similarity was very high within clade C (99.6-100\% between Symbiodinium $\mathrm{C} 1$ and $\mathrm{C} 3$; 91\% for $\mathrm{C} 15$ against $\mathrm{C} 1$ and C3). On the other hand, the highest sequence variation at the amino acid level was found between types A1 (Locus 9887) and F1 (Mv), with only 62\% similarity over 359 residues (Appendix Table 7.1). Residues involved in binding of heme and hydrogen peroxide, as well as the formation of the catalytic sites, were highly conserved except for those in Symbiodinium C15 and F1, where $\operatorname{Trp}^{161}$ and His ${ }^{162}$ in the hydrogen peroxide binding site were replaced by $\mathrm{Phe}^{161}$ in $\mathrm{C} 15$ and $\mathrm{Asn}^{162}$ in both (Figure 3.5). A total of 6 out of 13 residues involved in the binding of ascorbate and heme were also different in Symbiodinium $\mathrm{C} 15$, relative to the other types. Phylogenetic analysis of the APX amino acid sequences indicated a highly supported cladal separation between Symbiodinium clades $A, B$ and $C+F$, with clade $A$ at the base of the Symbiodinium cluster (Figure 3.6). The Symbiodinium cluster was most closely related to a cluster containing sequences of Thalassiosira sp. (Bacillariophyceae) and Emiliania huxleyi, (Prymnesiophyceae), which also contained one of the Symbiodinium A sequences. Another cluster of species from these two algal classes was also closer related to Symbiodinium than sequences from other dinoflagellates, rhodophytes, chlorophytes and embryophytes (land plants) (Figure 3.6).

[next page]

Figure 3.5. Alignment of ascorbate peroxidase protein sequences.

Protein alignment of ascorbate peroxidase (APX) sequences of different Symbiodinium types with annotation of functionally important residues. Indicated are residues involved in binding of heme (red), ascorbate (dark green) and cations (proximal [yellow] and distal [dark blue]). Binding sites for hydrogen peroxide (light blue) and residues involved in the formation of the catalytic site (grey) are highlighted. Annotations were deducted from alignments with characterized sequences from Pitsch et al. (2010). Culture ID, host and accession numbers are given in brackets. Colour coding for sequence similarities as in Figure 3.1. 


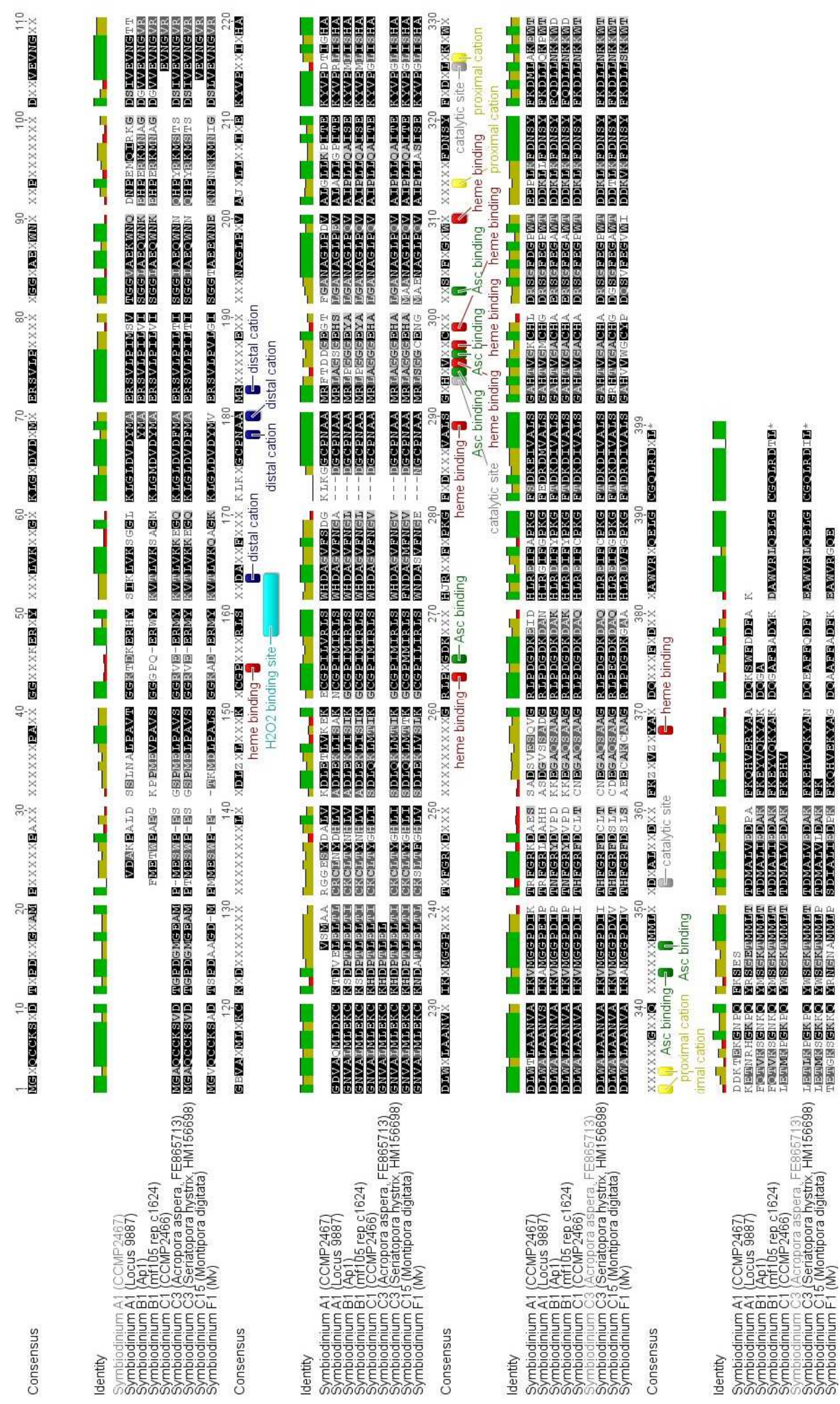




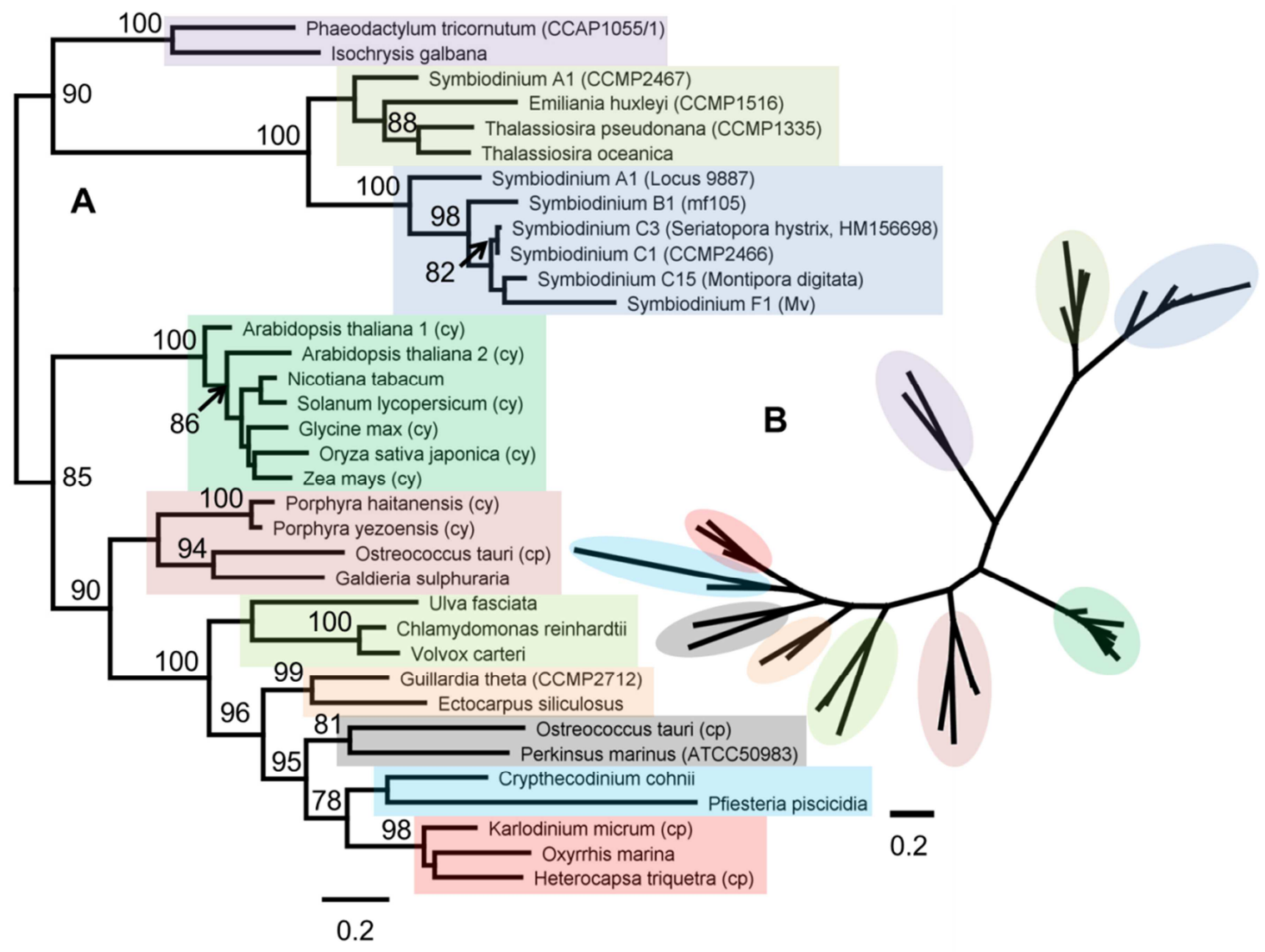

Figure 3.6. Ascorbate peroxidase protein phylogeny.

Ascorbate peroxidase (APX) phylogeny based on protein sequences from Symbiodinium sequences and other taxa inferred from maximum likelihood analysis as rooted $(A)$ and unrooted trees (B). Culture ID, host or accession numbers are given in brackets, and where available, the cytosolic (cy) or chloroplastic (cp) form of APX is indicated. Scale bars reflect the number of amino acid substitutions, and bootstrap percentages $>70 \%$ are indicated on nodes. Location of clusters in the unrooted tree is indicated by colour.

\section{Catalase peroxidase}

The Symbiodinium A1 (CCMP2467) and B1 (Ap1) sequences amplified here were identical to the A1 EST entry (CCMP2467 Locus 513; overhang used for alignment) and the assembled B1 (mf105) EST sequences (contigs mf105_rep_c169, mf105_rep_c11051, mf105_rep_c35625). The full length of the KatG protein for Symbiodinium B1 was 486 aa $(54.4 \mathrm{kDa})$ with a predicted isoelectric point of 6.28. KatG sequences from a total of eight different Symbiodinium genotypes were used for the protein alignment (Figure 3.7). The proximal heme-ligand signature motif (TVALIGGGHTL; Prosite PS00435) was highly conserved in all types and for the three sequences that contained the 
complete N-terminal domain (types B1, C1, C3), a signal peptide (aa 1-17) for the secretory pathway was found (but with low reliability class 5 ). Overall identity at the amino acid level between clades B, C, E and F was $85-87 \%$ (over a comparable length), and $\sim 70-80 \%$ for clade $A$ and $D$ (over a shorter alignment area). Symbiodinium $\mathrm{C} 1$ and $\mathrm{C} 3$ shared the highest pairwise identity with $98.8 \%$.

Comparison with bacterial KatG revealed that KatGs from Symbiodinium (SymKatG) are generally shorter and cover only the $\mathrm{N}$-terminal domain, with types $A 1, B 1$ and $D$ having a stop codon at approximately the same position. Residues of the heme-binding site within this domain are, however, almost all conserved (Figure 3.7, Appendix Figure 7.5). Structural modelling of the SymKatG from B1 (mf105) identified the crystal structure of the bacterial catalase-peroxidase from Haloarcula marismortui (PDB ID 1itk) as the best structural analog (TM-score $=0.839)$. In comparison to bacterial and other dinoflagellate KatGs, SymKatGs contain three large inserts at positions 217-236, 264-276 and 327-340 (Appendix Figure 7.5). Superimposition of the SymKatG from B1 (mf105) with Haloarcula marismortui (PDB ID 1itk) indicates that these inserts form large loops, primarily on the surface of the protein (Figure 3.8). Inserts 2 and 3 were also the main source of sequence variability between Symbiodinium types, with multiple amino acid inserts or deletions, especially in Symbiodinium B1.

Phylogenetic analysis located the Symbiodinium cluster near other dinoflagellate lineages, but distantly from bacterial sequences. Symbiodinium B1 was identified as ancestral to clade $\mathrm{C}$, while $\mathrm{C} 1$ and $\mathrm{C} 3$ could be distinguished phylogenetically within clade $\mathrm{C}$ (Figure 3.9). 


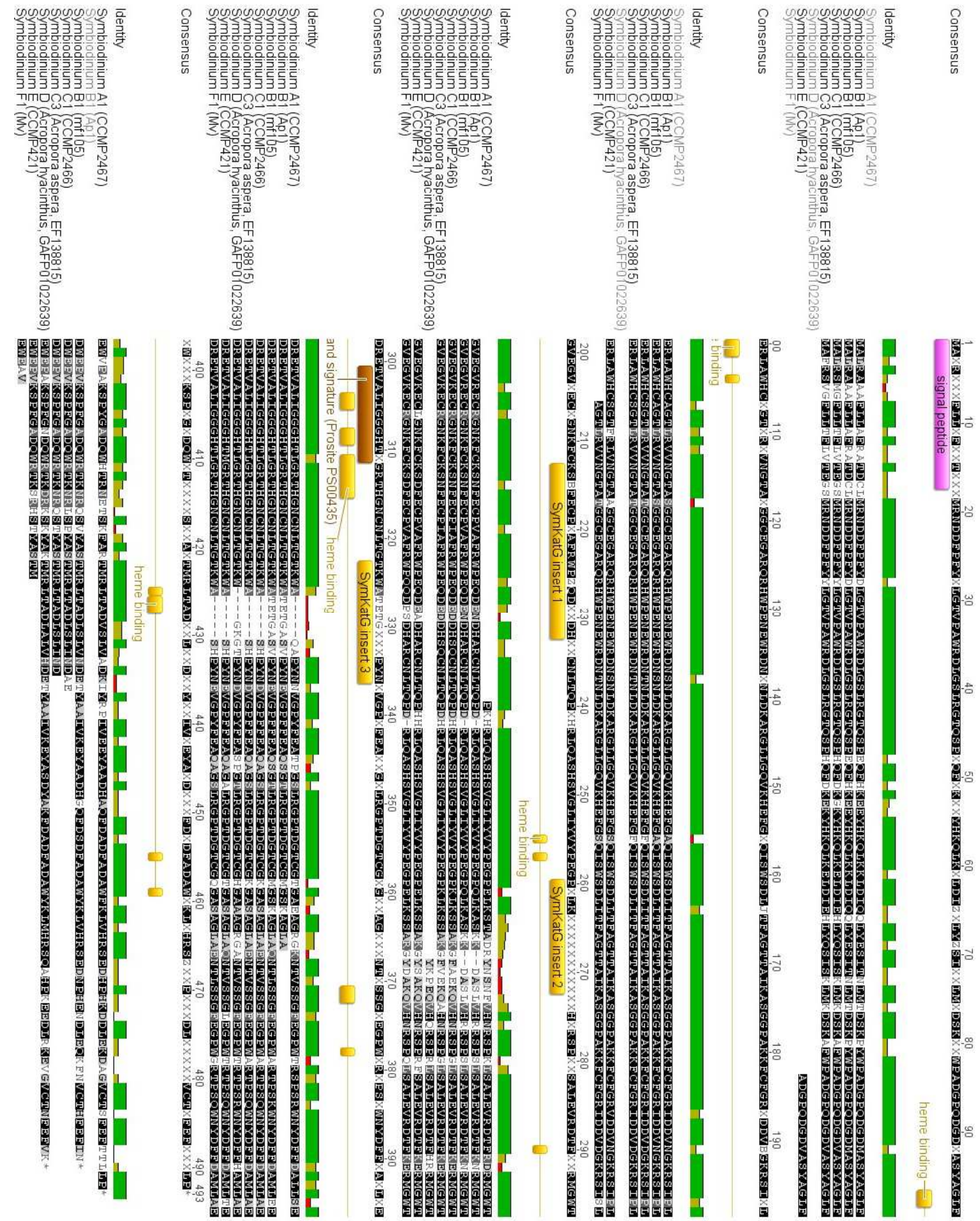

Figure 3.7. Catalase peroxidase protein alignment.

Protein alignment of catalase peroxidase (KatG) sequences of Symbiodinium types with annotation of a signal peptide (magenta), location of Symbiodinium-specific inserts relative to bacterial sequences and residues involved in heme binding (yellow). Annotations are based on alignments with annotated crystal structures of KatG (cf. Appendix Figure 7.5). Location of proximal heme ligand signature is indicated (brown). Culture ID, host and accession number are given in brackets. Colour coding for sequence similarities as in Figure 3.1. 


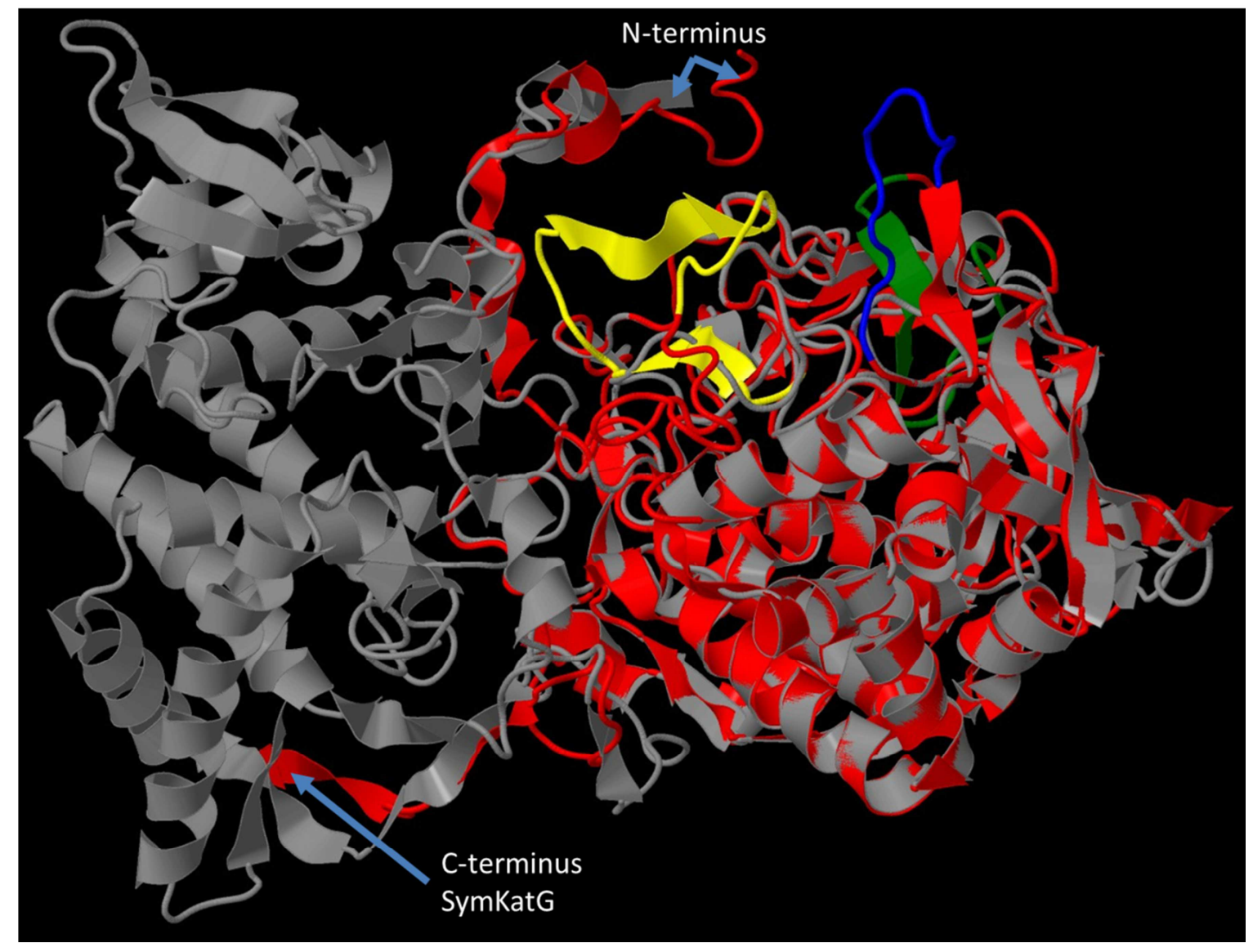

Figure 3.8. Structural comparison of the catalase peroxidase from the bacterium Haloarcula marismortui and Symbiodinium B1.

Superimposition of predicted mature catalase peroxidase (KatG) protein from Symbiodinium B1 $(\mathrm{mf} 105, \mathrm{C}$-score $=0.09)(\mathrm{red})$ with the crystal structure monomer from Haloarcula marismortui (PDB ID 1itk, grey), showing the lack of the C-terminal domain in the Symbiodinium KatG (SymKatG) relative to the bacterial form. Location of the typical loop inserts 1 (yellow), 2 (blue) and 3 (green) in Symbiodinium is highlighted (see text, cf. Appendix Figure 7.5). 


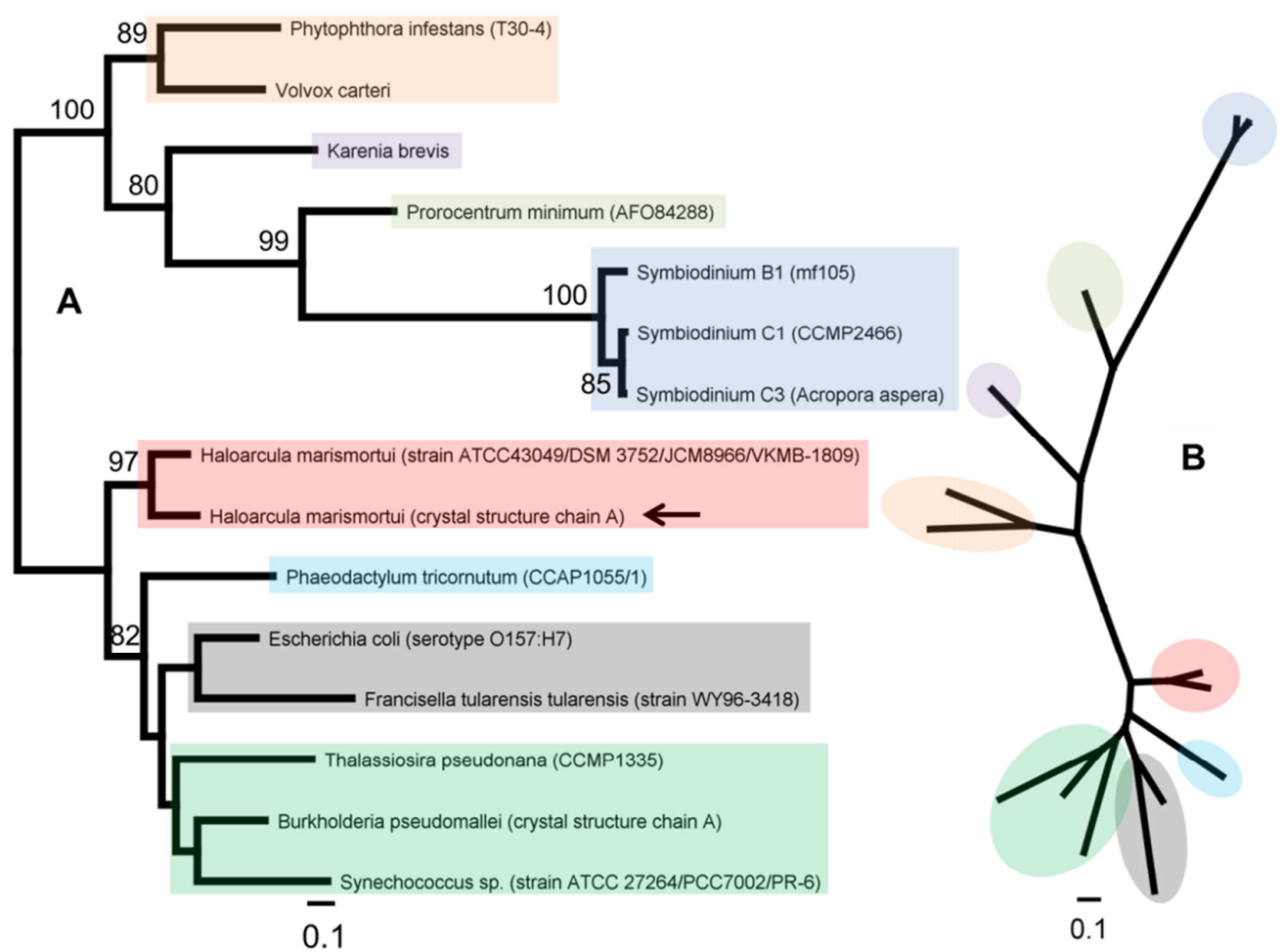

Figure 3.9. Catalase peroxidase protein phylogeny.

Catalase peroxidase (KatG) phylogeny of Symbiodinium types and other eukaryotic and bacterial taxa, inferred from maximum likelihood analysis as rooted (A) and unrooted trees (B). Only the $\mathrm{N}$-terminal domain was used for the alignment (cf. Appendix Figure 7.5). Location of the bacterial sequence used for the structural alignment (Figure 3.8) is indicated by an arrow. Culture ID, host or accession numbers for all other sequences is given in brackets. Scale bars reflect the number of amino acid substitutions, and bootstrap percentages $>70 \%$ are indicated on nodes. Location of clusters in unrooted tree is indicated by colour.

\section{Viability and proteomic temperature response of Symbiodinium B1}

Exposure to $33^{\circ} \mathrm{C}$ over three days led to an arrest in cell growth, relative to the control $(\mu=0.13 \pm 0.04$, mean $\pm S E, N=6)$, and a $16 \%$ decline in $F_{v} / F_{m}$ (Table 3.2). This was, however, not accompanied by a decline in chlorophyll a content. While no significant effect of temperature on protein content was found over time, values on Day 3 tended to be higher at $33^{\circ} \mathrm{C}(\mathrm{p}=0.0733)$. A significant temperature effect on antioxidant enzyme activity was only found for APX activity, where activities at $33^{\circ} \mathrm{C}$ were approximately $30 \%$ higher after three days (Table 3.2). 
Table 3.2. Temperature effects on viability and physiological variables in Symbiodinium B1.

Shown are results for viability, protein and chlorophyll a content, and the activity of enzymatic antioxidants in Symbiodinium B1 in culture over three days of exposure to $25^{\circ} \mathrm{C}$ and $33^{\circ} \mathrm{C}$. Values are means $\pm \mathrm{SE}(\mathrm{N}=6)$. Statistical effects are based on rmANOVA results. Significant effects are indicated by asterisks.

\begin{tabular}{|c|c|c|c|c|c|}
\hline Variable & ${ }^{\circ} \mathrm{C}$ & Day 0 & Day 1 & Day 3 & $F_{\text {time } \mathrm{x} \text { temperature }}$ \\
\hline \multirow{2}{*}{$\begin{array}{c}\text { Cell } \\
\text { density }\end{array}$} & 25 & $148920 \pm 6142$ & $161651 \pm 6553$ & $216050 \pm 10864$ & \multirow{2}{*}{$\begin{array}{c}F_{2,9}=40.3486 \\
p<0.0001^{*}\end{array}$} \\
\hline & 33 & $151759 \pm 11152$ & $128704 \pm 6988$ & $126389 \pm 5798$ & \\
\hline \multirow{2}{*}{$\mathrm{F}_{\mathrm{v}} / \mathrm{F}_{\mathrm{m}}$} & 25 & $0.51 \pm 0.01$ & $0.51 \pm 0.01$ & $0.51 \pm 0.01$ & \multirow{2}{*}{$\begin{array}{c}F_{2,9}=19.5472 \\
p=0.0005^{*}\end{array}$} \\
\hline & 33 & $0.51 \pm 0.02$ & $0.46 \pm 0.02$ & $0.43 \pm 0.01$ & \\
\hline \multirow{2}{*}{$\begin{array}{l}\text { Protein } \\
{\left[\text { pgg cell }^{-1}\right]}\end{array}$} & 25 & $3.82 \pm 0.31$ & $4.62 \pm 0.40$ & $4.67 \pm 0.28$ & \multirow{2}{*}{$\begin{array}{c}F_{2,9}=2.9263 \\
p=0.1049\end{array}$} \\
\hline & 33 & $4.74 \pm 0.46$ & $4.23 \pm 0.38$ & $5.57 \pm 0.35$ & \\
\hline \multirow{2}{*}{$\begin{array}{c}\text { Chl a } \\
{\left[\text { pg cell }^{-1}\right]}\end{array}$} & 25 & $0.85 \pm 0.05$ & $0.89 \pm 0.03$ & $0.77 \pm 0.05$ & \multirow{2}{*}{$\begin{array}{c}F_{2,9}=1.2197 \\
p=0.3398\end{array}$} \\
\hline & 33 & $0.90 \pm 0.03$ & $1.00 \pm 0.06$ & $0.92 \pm 0.08$ & \\
\hline \multirow{2}{*}{$\begin{array}{c}\text { SOD } \\
{\left[\mathrm{nU} \mathrm{cell}^{-1}\right]}\end{array}$} & 25 & $389.6 \pm 25.7$ & $453.8 \pm 48.8$ & $541.0 \pm 33.4$ & \multirow{2}{*}{$\begin{array}{c}F_{2,9}=1.7356 \\
p=0.2304\end{array}$} \\
\hline & 33 & $486.3 \pm 44.4$ & $437.9 \pm 37.9$ & $594.1 \pm 34.3$ & \\
\hline \multirow{2}{*}{$\begin{array}{c}\text { APX } \\
{\left[n U \text { cell }^{-1}\right]}\end{array}$} & 25 & $2.01 \pm 0.22$ & $2.23 \pm 0.26$ & $2.31 \pm 0.22$ & \multirow{2}{*}{$\begin{array}{c}F_{2,9}=4.2666 \\
\mathrm{p}=0.0497^{\star}\end{array}$} \\
\hline & 33 & $2.17 \pm 0.24$ & $2.14 \pm 0.25$ & $2.99 \pm 0.29$ & \\
\hline \multirow{2}{*}{$\begin{array}{c}\text { KatG } \\
{\left[\mathrm{nU} \mathrm{cell}^{-1}\right]}\end{array}$} & 25 & $17.43 \pm 3.69$ & $15.11 \pm 2.93$ & $13.77 \pm 1.95$ & \multirow{2}{*}{$\begin{array}{c}F_{2,9}=0.7105 \\
p=0.5170\end{array}$} \\
\hline & 33 & $19.86 \pm 2.58$ & $20.74 \pm 3.34$ & $19.45 \pm 2.30$ & \\
\hline
\end{tabular}

\section{Antioxidant gene expression in Symbiodinium B1 under thermal stress}

The reference genes Cox and $\mathrm{Cal}$ were identified as the best combination of $\mathrm{HKG}$, with a combined stability value of 0.006 and 0.008 at $25^{\circ} \mathrm{C}$ and $33^{\circ} \mathrm{C}$, respectively (Figure 3.10A). Relative expression of the remaining house-keeping genes and the target genes at $33^{\circ} \mathrm{C}$ showed no significant change over time. However, $S A M$ and $M n S O D$ exhibited significantly higher expression at $33^{\circ} \mathrm{C}$, relative to $25^{\circ} \mathrm{C}$, on Day 0 (Figure $3.10 \mathrm{~B}$ ). The high variability in relative expression for most of the genes on Days 0 and 1 had disappeared by Day 3 , where all replicates had a similar range of expressions. These were, however, not significantly different from the control treatment. 

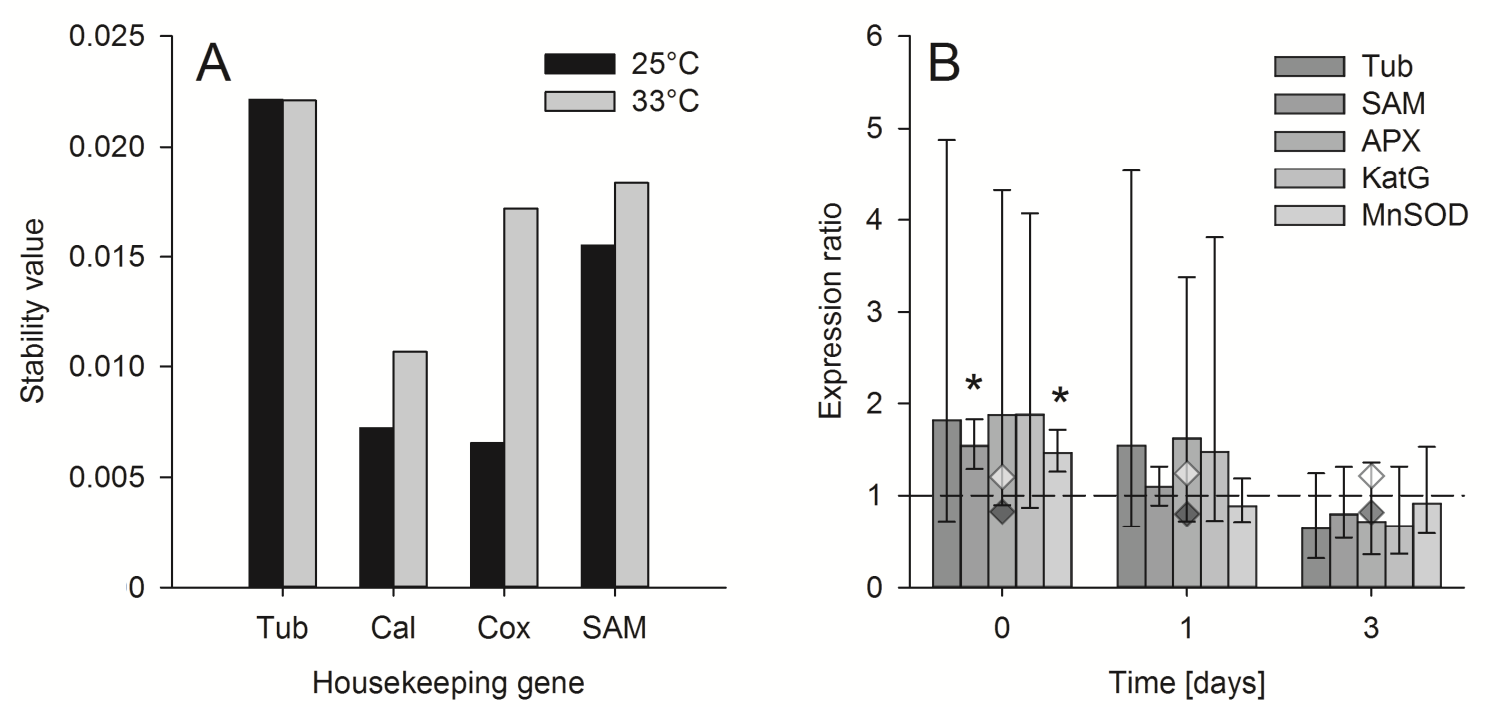

Figure 3.10. Stability of HKG and gene expression in Symbiodinium B1.

(A) Expression stability of potential housekeeping genes at $25^{\circ} \mathrm{C}$ and $33^{\circ} \mathrm{C}$ over three days exposure to $25^{\circ} \mathrm{C}$ and $33^{\circ} \mathrm{C}$ in Symbiodinium $\mathrm{B} 1(\mathrm{~N}=6)$. (B) Expression of antioxidant and unsuitable HKG in Symbiodinium $\mathrm{B} 1$ at $33^{\circ} \mathrm{C}$ relative to control. Shown are expression ratios for B-tubulin (Tub), S-adenosyl methionine synthetase (SAM), ascorbate peroxidase $(A P X)$, catalase peroxidase $(K a t G)$ and manganese superoxide dismutase $(M n S O D)$. Values are means $\pm \mathrm{SE}(\mathrm{N}=6)$. Expression of the HKG calmodulin (white diamonds) and cytochrome oxidase subunit 1 (black diamonds) is indicated. Asterisks indicate significant upregulation relative to the control $(p<0.05)$.

\section{Discussion}

This study provides, for the first time, an overview of the intraspecific genetic diversity of antioxidant genes in the genus Symbiodinium. Sequence characteristics and phylogenetic analyses indicated a high degree of sequence conservation at the amino acid level for SOD isoforms, but considerable variation in peroxidases within the genus. Gene expression and proteomic activity of MnSOD, APX and KatG were successfully measured in Symbiodinium B1 at different temperatures. Significant changes in APX activity could however not be related to changes in gene expression.

\section{SOD isoforms are highly conserved between Symbiodinium types}

Superoxide dismutases are an important component of the antioxidant system that detoxifies ROS in higher plants (Alscher et al., 2002). Activity of these enzymes has also been shown to be a fundamental part of the thermal response of the photosynthetic coral symbiont, Symbiodinium sp. The large diversity in this dinoflagellate genus has been discovered on the basis of the genetic ITS2 
marker (LaJeunesse, 2001). However, to what degree this genetic diversity is reflected in functionally important genes, such as antioxidant genes, has scarcely been investigated (Pochon et al., 2014). While previous studies have established the presence of all three SOD isoforms (CuZnSOD, MnSOD, FeSOD) in Symbiodinium (Lesser \& Shick, 1989a, Matta et al., 1992, Richier et al., 2003), the random primer approach used here detected only the manganese and iron SOD forms. At least three MnSOD isoforms exists in Symbiodinium B1, and these findings agree with the electrophoretic detection of three to four MnSOD isoforms in Symbiodinium from two different host species (Richier et al., 2003). Interestingly, no FeSOD sequence was found in any of the EST libraries. This could be related to the fact that FeSOD seems to be expressed at a very low level, as high $\mathrm{C}_{\mathrm{t}}$-values and problems with non-specific amplification in the GPCR suggest. The difficulties encountered in amplifying FeSOD prevent conclusions being made about the true diversity of this form, though at least two isoforms were reported for the symbionts of the sea anemone Anemonia viridis (Richier et al., 2003). This study confirms, however, that FeSODs from several Symbiodinium types are indeed dimeric and the molecular weight of $21.9 \mathrm{kDa}$ for a Symbiodinium A clade member matches previous findings (Matta et al., 1992).

Structurally, all Symbiodinium MnSODs found here are dimeric, a characteristic that is generally found in prokaryotes rather than eukaryotes, where MnSODs are usually tetrameric (Halliwell \& Gutteridge, 2007). Though signal peptides and cleavage sites were detected in all MnSODs, the low reliability of the prediction using the "plant" setting in TargetP 1.1 requires further investigation with regards to the subcellular locations of these forms. Similarly, the absence or low reliability of a chloroplastic target signal in photosynthetic and heterotrophic dinoflagellate FeSODs from Symbiodinium A (PF-2005, AY916504), Crypthecodinium cohnii (ABQ23892) and Lingulodinium polyedrum (AF289824) emphasizes the need for such investigations, but also raises an important question: are plastid targeting signals in dinoflagellates considerably different from the ones in higher plants, or do these FeSODs represent cytosolic forms as suggested previously for heterotrophic dinoflagellates (Dufernez et al., 2008)?

Sequence homology between Symbiodinium types was very high for MnSOD and FeSOD. As a result, these enzymes are structurally almost identical, suggesting 
similar catalytic properties between types. This homology is also reflected in the fact that it was not possible to phylogenetically distinguish clades within the Symbiodinium cluster for MnSOD. This high sequence similarity at the amino acid level was probably the reason why various members of the evolutionary sister groups of alveolates (with dinoflagellates) and stramenopiles were not clustered according to their taxonomy. Indeed, the Symbiodinium cluster was more related to the stramenopile Aureococcus anophagefferens and to all other stramenopiles than to the dinoflagellate Noctiluca scintillans. In fact, the patristic distance from Symbiodinium B1 to $N$. scintillans and E. coli was approximately the same.

\section{C-terminal reduction and additional loops characterize Symbiodinium catalase peroxidases}

Catalase peroxidases (KatG) are bifunctional heme peroxidases which, in contrast to other peroxidases, express both catalase and peroxidase activity. These enzymes are of bacterial origin and resulted from a gene duplication event, in which both protein domains remained fused while the C-terminal domain lost its catalytic activity (Welinder, 1991). KatGs in Symbiodinium (SymKatG) are characterized by two major structural alterations in comparison to the bacterial form. One of them is the fact that SymKatGs have completely lost their C-terminal domain. This reduction or absence of the C-terminal domain has been observed in other dinoflagellates before (Guo \& Ki, 2013). The exact function of this domain in bacterial KatGs is, however, unknown. It is suspected to stabilize the protein, while its absence affects the spatial layout of the heme-binding residues in the catalytic domain (Baker et al., 2004, Zámocký et al., 2010).

The second feature of SymKatGs is the considerable extension of three KatGtypical loops, relative to bacterial and other phytoplankton groups. The high sequence variability between Symbiodinium types in Loop 2 (SymKatG Insert 1) might be evolutionarily important, since Loops 1 and 2 are structurally relevant for the access of hydrogen peroxide to the prosthetic heme group and thus catalytic activity (Li \& Goodwin, 2004, Smulevich et al., 2006). In addition, substitutions of residues involved in heme and hydrogen peroxide binding, as found in Symbiodinium F1, directly affect the properties of this enzyme and are therefore relevant for type-specific differences in KatG activity and hydrogen peroxide scavenging. 
Sequence evolution of peroxidases reflect patterns of genetic radiation in the genus Symbiodinium

Ascorbate peroxidase (APX) and catalase peroxidase (KatG) transcripts expressed a greater number of amino acid substitutions between Symbiodinium types than did superoxide dismutase transcripts, which could indicate a higher rate of sequence evolution for these two phylogenetically-related heme peroxidases. Both, APX and KatG phylogeny reflected the evolutionary phylogeny of Symbiodinium within the range of types tested (Pochon \& Gates, 2010). It was even possible to resolve closely related members such as $C 1$ and C3 (the ancestral core of the C clade) within the KatG phylogeny. It is therefore conceivable that this enzyme has co-evolved with the genetic radiation of the genus. The inter-cladal sequence divergence of APX (with similarities as low as $62 \%$ between types $A 1$ and $F 1$ ) observed in the phylogenetic analysis, is in fact much higher than the one seen at the genus level of higher plants. While this coevolution of peroxidases within the genus is evident, the phylogenetic analysis suggests different origins of these genes. For APX, all sequences were phylogenetically closer to clusters containing members of the Bacillariophyceae and Haptophyta, rather than grouping with other photosynthetic and nonphotosynthetic dinoflagellates. This could either suggest a common source of APX-coding genes or the acquisition of these genes through horizontal gene transfer from either group, as has been shown for photosynthetic genes in the dinoflagellate Karlodinium venificum (Patron et al., 2006). In contrast, while SymKatGs are similar to those of other dinoflagellates, they show a larger evolutionary distance from their bacterial origin than from the KatGs of Bacillariophyceae. Horizontal gene transfer of KatGs from cyanobacteria or marine bacteria has been suggested for diatoms (Zámocký et al., 2010). Diatom sequences do not, however, express the degree of C-terminal reduction observed in dinoflagellates. The suggested common KatG ancestry of dinoflagellates with Volvox carteri and Phytophthora infestans in this analysis (including the C-terminal reduction) could suggest multiple independent gene transfer events for KatGs. Both enzymes are nuclear encoded, as the presence of a DinoSL trans-splicing mechanism suggests (Zhang \& Lin, 2009, Guo \& Ki, 2013). Attempts in the current study to amplify the N-terminal domain of APX via the DinoSL primer were, however, only successful for Symbiodinium F1. Based 
on the absence of a signal peptide in this type, it is likely that the isoform here represents a cytosolic APX. While it is not directly involved in chloroplastic ROS scavenging, it has a pivotal role in the protection of mitochondrial and chloroplast compartments by regulating cytosolic hydrogen peroxide, as shown for higher plants such as Arabidopsis thaliana (Karpinski et al., 1997, Davletova et al., 2005).

In dinoflagellates, processes of secondary and tertiary endosymbiosis with the retainment of plastids contributed to the incorporation of genes from other eukaryotic groups via horizontal gene transfer (reviewed in Wisecaver \& Hackett, 2011). Symbiodinium is certainly no exception (Rowan et al., 1996, Takishita et al., 2003a, Leggat et al., 2007), and the genetic radiation and varying proximity of both types of peroxidases to phylogenetic sister groups supports an early acquisition of these genes. On the other hand, a high degree of conservation in SODs shows the limitation of genetic variation, as mutations to critical residues of structural characteristics and catalytic functions might be deleterious in these enzymes (Cooper \& Brown, 2008).

\section{Antioxidant gene expression in Symbiodinium B1}

In order to normalize antioxidant expression, a number of potential HKGs were chosen that had previously been shown to be suitable for thermal experiments with Symbiodinium C3 (Rosic et al., 2011b). The Normfinder analysis for expression stability agreed with this previous study, in that Tub is the least stable HKG candidate for thermal stress, however it also indicated that in Symbiodinium $\mathrm{B} 1$, in contrast to $\mathrm{C} 3, \mathrm{Cal}$ and Cox are more stable than SAM. Large variations in gene expression between replicates were evident in the current study. Though natural variation in gene expression has been shown for in hospite Symbiodinium under field conditions (Leggat et al., 2011), there is no obvious explanation as to why this variation was present under the controlled lab settings, and why it was less prominent in SAM and MnSOD expression. Changes in gene expression in Symbiodinium under stress have consistently been found to be small (Boldt et al., 2008, Leggat et al., 2011, Rosic et al., 2011a), thus minimizing variation between replicates is crucial to resolve minor responses. 
Clearly, the thermal scenario tested here arrested growth and lowered the maximum quantum yield in Symbiodinium B1. It was, however, insufficient for evoking a significant response in the expression and activity of most of the antioxidants monitored here. Indeed, only cellular hydrogen peroxide scavenging through APX activity was significantly elevated after three days at $33^{\circ} \mathrm{C}$ in Symbiodinium B1. Considering the lack of response, and the fact that PAM values declined only moderately (by 16-17\%), it is likely that severe photodamage as an initiator of oxidative stress in Symbiodinium B1 might not have occurred yet (Chapter 2; Iglesias-Prieto et al., 1992, Lesser, 1996). However, elevated APX activity might indicate the beginning of an increased turnover of hydrogen peroxide via the Foyer-Halliwell-Asada cycle, which is usually linked to photosynthetic processes of alternative energy dissipation, such as the Mehler reaction (the univalent reduction of oxygen as an alternative electron acceptor at photosystem I) (Asada, 2000). Due to the lack of any significant enzymatic response within the timeframe tested, and the high degree of variation in gene expression between replicates, it was not possible to correlate changes in antioxidant gene transcripts and enzymatic activity in Symbiodinium B1 in the current study. Further research about the level of regulation of these important antioxidant genes in Symbiodinium under stress is needed, as some studies highlight the importance of post-translational regulation in dinoflagellates (Milos et al., 1990, Fagan et al., 1999, Okamoto et al., 2001). The sequence data provided here, and their successful application to measurements of antioxidant gene expression in Symbiodinium B1, provide the foundation for such studies. 
-80 - 


\section{Differential antioxidant activity in the coral holobiont under thermal stress}

\section{Introduction}

A coral colony represents a symbiome that consists of a number of cohabiting taxa. The most important association in reef-building zooxanthellate corals is the endosymbiosis with dinoflagellates of the genus Symbiodinium, which enhances calcification rates and facilitates survival in nutrient-poor waters by providing photosynthetically fixed carbon to the host and promoting the conservation and recycling of nitrogen (Muscatine \& Cernichiari, 1969, Muscatine \& Porter, 1977, Wang \& Douglas, 1997). For these reasons, coral reefs would not exist without this symbiosis. The disruption of this obligate symbiosis by environmental stress has been termed coral bleaching, which is phenotypically visible as a paling of the coral tissue resulting from a loss of the dinoflagellate symbionts and/or their photosynthetic pigments (Gates et al., 1992, Iglesias-Prieto \& Trench, 1994). With the onset of anthropogenic marine pollution and climate change, many reef systems have experienced major perturbations and regime shifts, often associated with bleaching (Pandolfi et al., 2003, Knowlton \& Jackson, 2008). Indeed more than $16 \%$ of the world's coral reefs have been effectively lost as a result of these processes (Wilkinson, 2008). Today, ocean warming is one of the main global challenges faced by reef systems.

Thermal thresholds and mortality vary between coral species (Marshall \& Baird, 2000, Loya et al., 2001, Stimson et al., 2002). The reason for this differential bleaching susceptibility is, to a certain extent, a result of the specific holobiont composition, i.e. the combination of different host species and one or multiple Symbiodinium genotypes (Rowan et al., 1997, Loram et al., 2007, Abrego et al., 2008, Sampayo et al., 2008). In addition, physiological acclimation (e.g., IglesiasPrieto \& Trench, 1994, Gates \& Edmunds, 1999, Middlebrook et al., 2008), host genetic background (Smith-Keune \& van Oppen, 2006, Barshis et al., 2010), and symbiont effects on host physiology (Rowan, 2004a, Ulstrup et al., 2007, Abrego et al., 2008), as well as host heterotrophy during episodes of bleaching and recovery (Grottoli et al., 2004, Grottoli et al., 2006), are important factors that 
define the stability and resilience of the coral symbiosis to environmental stressors. However, the complex interaction of these factors and the resulting trade-offs make an ecological assessment about the relative importance of each partner difficult (Fitt et al., 2001, Berkelmans \& van Oppen, 2006, Abrego et al., 2008, Fitt et al., 2009, Jones \& Berkelmans, 2010). Moreover, while some intracellular sites and effects of thermal damage in the host and symbiont have been identified, there is no consensus about the causal chain of events and the 'weak link' in the symbiosis. Indeed, bleaching can be manifested as host-cell detachment and/or release of potentially photosynthetically-competent symbionts (Gates et al., 1992, Ralph et al., 2001), as well as breakdown of the photosynthetic dark and light reactions (Jones et al., 1998, Warner et al., 1999), and apoptosis and necrosis in both partners (Dunn et al., 2002, Dunn et al., 2004, Dunn et al., 2007b, Takahashi et al., 2008, Tchernov et al., 2011, Hawkins et al., 2013, Paxton et al., 2013).

One key process, suggested over 20 years ago by Lesser et al. (1990), is the leakage of reactive oxygen species (ROS) from the symbiont to the host, which then challenges and potentially overwhelms the host's antioxidant capacity (Downs et al., 2002, Venn et al., 2008, Weis, 2008). In addition, the presence of elevated levels of ROS as well as reactive nitrogen species (RNS) in the host tissue could interfere with innate immune pathways and trigger pro-apoptotic processes in the host (Perez \& Weis, 2006, Weis, 2008, Hawkins et al., 2013). The involvement of ROS in coral bleaching led to the proposal of a unifying mechanistic model of coral bleaching: the "Oxidative Theory of Coral Bleaching". Briefly, exposure to elevated temperature initially affects metabolic rates and growth of the symbiont at sub-lethal temperatures, and leads to reduced photosynthate translocation to the host as a result of a decline in the photosynthetic function in the symbiont. Chronic exposure and excessive temperatures lead to the breakdown of photosynthesis, and the generation of ROS and RNS in the symbiont, with subsequent damage to the cellular infrastructure and the activation of apoptotic pathways in both partners (reviewed in Lesser, 2011).

There is a large body of literature that illustrates increased antioxidant activity as a result of thermal and irradiance stress in both symbionts and their hosts, 
supporting the idea that oxidative stress is the trigger of coral bleaching (reviewed by Lesser, 2006, Lesser, 2011). Studies, monitoring elements of the antioxidant network under thermal stress in both partners simultaneously have been conducted for the corals Galaxea fascicularis (adult), Acropora intermedia (larvae), Platygyra ryukyuensis (adult) and Stylophora pistillata (adult), as well as a number of anemones (Lesser et al., 1990, Yakovleva et al., 2004, Richier et al., 2005, Higuchi et al., 2008, Yakovleva et al., 2009, Higuchi et al., 2012). To what extent antioxidant capacities in each partner contribute to the overall bleaching susceptibility of the holobiont is an important question. In the context of the Oxidative Theory of Coral Bleaching, differential bleaching susceptibility of coral holobionts should be related to mechanisms that prevent or combat the excessive generation of ROS under thermal stress (Downs et al., 2002). Furthermore, if oxidative stress initiates the thermal bleaching cascade, then one should observe distinct temporal patterns of antioxidative defences in both partners. Identifying how the ROS-scavenging processes in the symbiont are linked to the host and whether they precede, coincide with or are independent of the host's response, is of fundamental importance for explaining specific thermal thresholds of different holobionts. Here, physiological data from three common Indo-Pacific corals with differing thermal susceptibilities are presented. The aim of this study was to connect phenotypic bleaching data with the activity of major enzymatic antioxidants in both partners under field conditions.

\section{Material and Methods}

\section{Experimental setup}

Fragments of the scleractinian corals Acropora millepora (cream morph), Pocillopora damicornis (pink morph) and Montipora digitata (green morph) were collected from five different colonies per species $(\mathrm{N}=5)$ on the Heron Island reef flat in Feb 2012 (Heron Island Research Station, HIRS, 2333'S, 151 54'E) under the GBR Marine Park permit G12/34800.1. Each colony provided coral explants that were haphazardly allocated to one control and two $60 \mathrm{~L}$ flow-through seawater tanks at HIRS (Figure 4.1); care was taken to ensure that the coral explants were not in contact with one another. Half of the explants were used for analysis of host cellular and apoptotic signalling (Hawkins et al., 2014), whereas 
the other half were used to assess the activity of enzymatic antioxidants (this study). Tanks were set-up as flow through systems with unfiltered seawater (1.3$1.5 \mathrm{~L} \mathrm{~min}^{-1}$ ) pumped from the reef flat. Each tank was equipped with a $500 \mathrm{~W}$ aquarium heater connected to a temperature controller (MX-1019, WEIPRO®), Guangdong, China; $\pm 1^{\circ} \mathrm{C}$ accuracy).

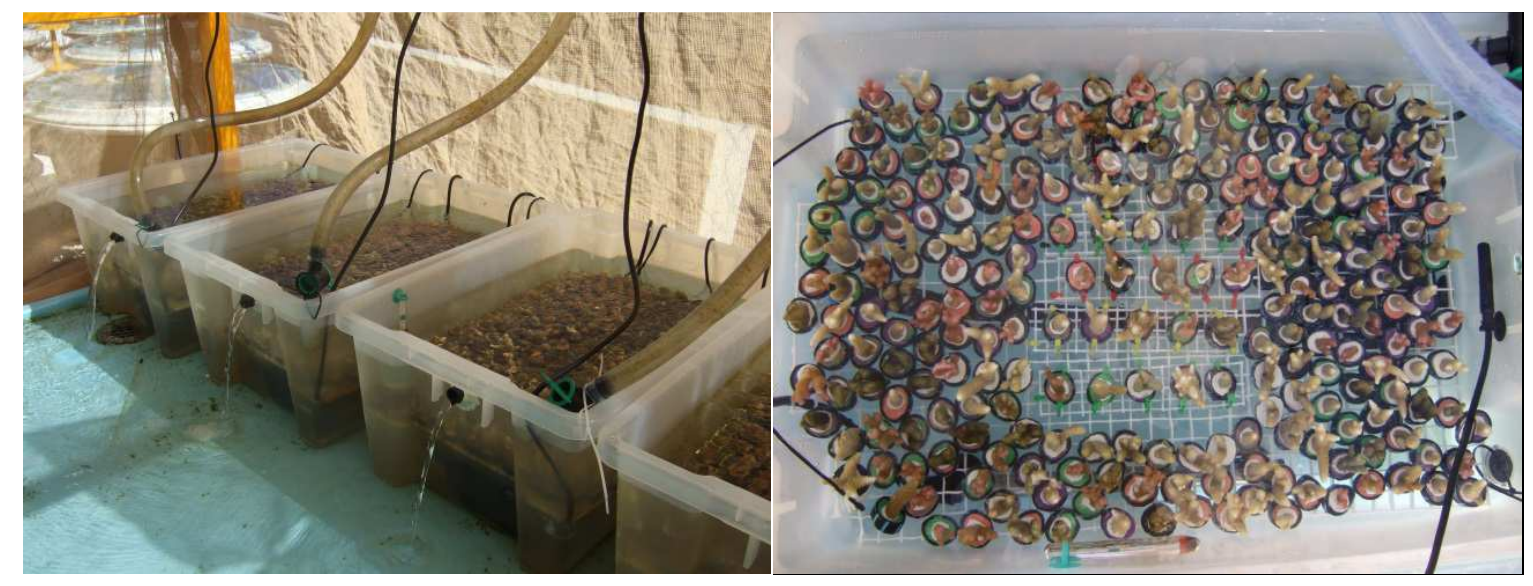

Figure 4.1. Experimental setup on Heron Island.

Tanks were set up as shaded flow-through systems (left) and individual explants fixed to plasticine mounts and placed on submerged trays (right).

The tanks were covered with shade-cloth to give $~ 25 \%$ of the natural irradiance throughout the experiment; this equalled $\sim 450-500 \mu \mathrm{mol}$ quanta $\mathrm{m}^{-2} \mathrm{~s}^{-1}$ on a clear sunny day at noon (LI-COR Quantum light meter LI-189 with cosine sensor, LICOR, Inc., Lincoln, NE, USA). Light data during the experimental period were recorded with an ODYSSEY PAR cosine recorder (DATAFLOW Systems Pty Ltd., Christchurch, New Zealand). This experimental design allowed for replicate corals under each thermal regime to be exposed to identical conditions. Water temperature in all tanks was maintained at a minimal temperature of $27.5^{\circ} \mathrm{C}$, based on long-term seawater temperature data for the Heron Island reef flat for February (AIMS, 2013) and monitored using HOBO Pendant ${ }^{\circledR}$ temperature/light data loggers (Onset Computer Corporation, Bourne, MA, USA). Corals were acclimated for nine days prior to temperature ramping. After sampling on Day 0 , two treatment tanks were heated by $1^{\circ} \mathrm{C}$ per day to their final average temperatures of $31.5^{\circ} \mathrm{C}\left( \pm 0.6^{\circ} \mathrm{C}\right)$ and $33^{\circ} \mathrm{C}\left( \pm 0.8^{\circ} \mathrm{C}\right)$. Average treatment temperature of the control was $28.5^{\circ} \mathrm{C}\left( \pm 0.9^{\circ} \mathrm{C}\right)$. Sampling occurred immediately after noon on Days 0, 5, 7 and 9 and explants were flash frozen in liquid nitrogen 
and stored at $-80^{\circ} \mathrm{C}$ until analysis. All tanks expressed a diel temperature cycle that was influenced by ambient conditions (Figure 4.2).

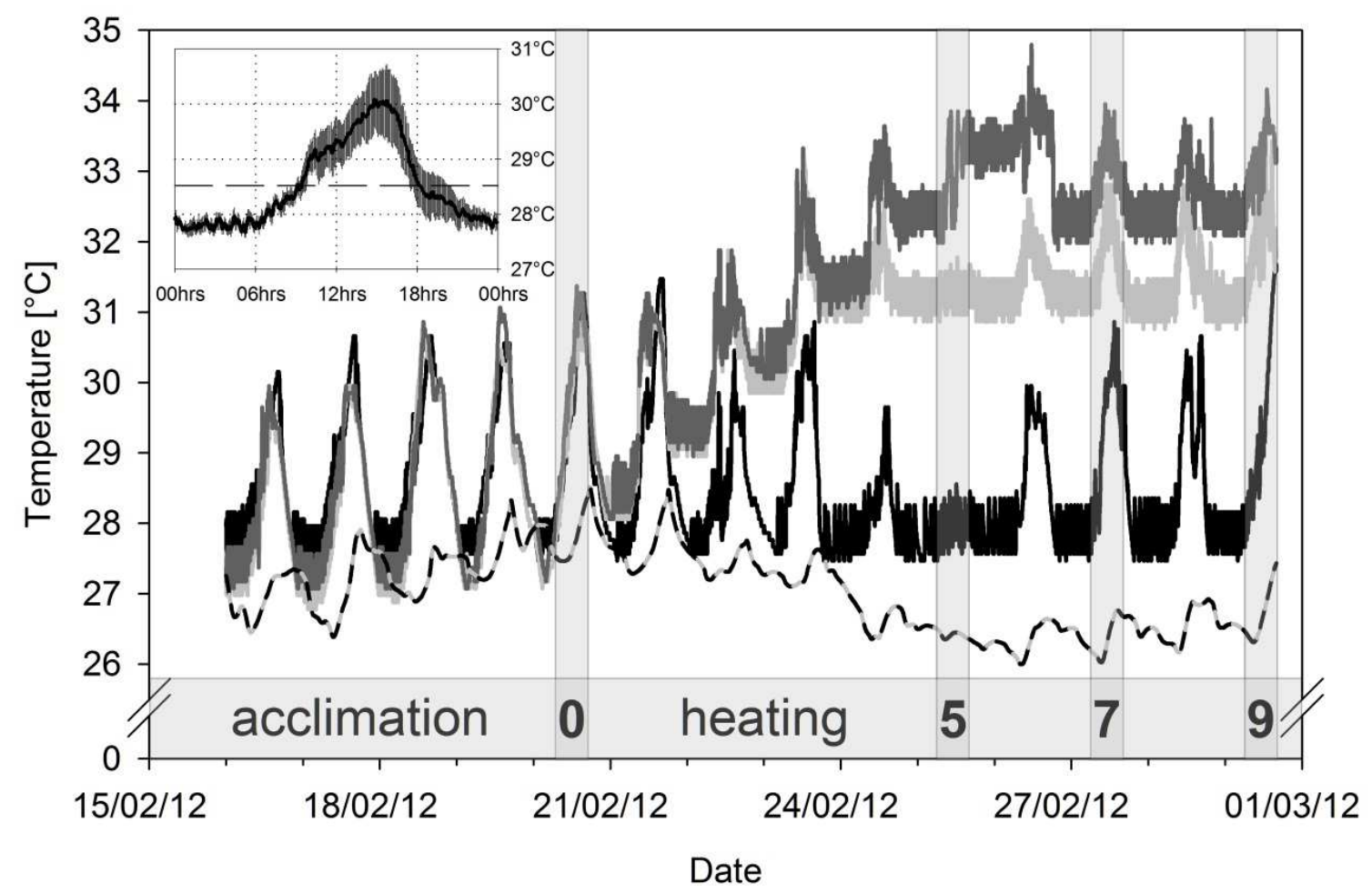

Figure 4.2. Temperature profile of experimental system.

Temperature profile of experimental treatments at $28^{\circ} \mathrm{C}$ (black), $31^{\circ} \mathrm{C}$ (grey) and $33^{\circ} \mathrm{C}$ (dark grey) in a flow-through tank system, during acclimation (prior to Day 0) and the experimental period (with sampling on Days 0,5,7 and 9; time-points highlighted by shading). Ambient water temperature at $2.04 \mathrm{~m}$ water depth (Heron Island relay pole 3, AIMS 2013) depicted as black-grey dashed line. Average daily temperature variation in the control tank over the whole period is shown as a graph insert (mean $\pm 95 \% \mathrm{Cl}, \mathrm{N}=$ 3937 , with overall mean of $28.5^{\circ} \mathrm{C}$ shown as a dashed line).

\section{Sample processing}

Coral and Symbiodinium samples were processed and centrifuged at $4{ }^{\circ} \mathrm{C}$ and samples maintained on ice. Coral explants were water-picked with $8 \mathrm{~mL}$ of cold lysis buffer (50 mM phosphate, $0.1 \mathrm{mM}$ EDTA, 10\% [v/v] glycerol, pH 7.0). Three $500 \mu \mathrm{L}$ aliquots for chlorophyll a ( $\mathrm{Chl}$ a) analysis, genotyping and cell density determination were taken. Samples for cell counting were fixed with $10 \mu \mathrm{L}$ Lugol's solution and stored at $-20^{\circ} \mathrm{C}$. Pigment samples were centrifuged $(16000 \times \mathrm{g}, 5$ min) and the pellet frozen and stored at $-20^{\circ} \mathrm{C}$ in the dark. Following initial centrifugation ( $2000 \times \mathrm{g}, 5 \mathrm{~min}$ ) to pellet the remaining Symbiodinium cells, the supernatant containing the coral tissue was centrifuged again (16000 $\mathrm{g}, 5 \mathrm{~min})$ 
to remove any particulate matter, aliquoted, flash frozen in liquid nitrogen and stored at $-80^{\circ} \mathrm{C}$. The Symbiodinium pellets were flash frozen and stored at $-80^{\circ} \mathrm{C}$ for later analysis. Lysis of Symbiodinium pellets after four wash cycles $(2000 \times \mathrm{g}$, $5 \mathrm{~min}$ ) was performed in lysis buffer using $200 \mathrm{mg}$ glass beads $(710-1180 \mu \mathrm{m}$; Sigma-Aldrich) in a bead mill $\left(50 \mathrm{~Hz}, 3 \mathrm{~min}, 4^{\circ} \mathrm{C}\right.$, Qiagen tissue lyser, Qiagen N.V., Hilden, Germany). All cell lysates were centrifuged (16000 x g, $5 \mathrm{~min}$ ), and supernatants aliquoted and frozen at $-80^{\circ} \mathrm{C}$.

\section{Viability}

Effective and maximum quantum yields of algal PSII $\left(\Delta \mathrm{F} / \mathrm{F}_{\mathrm{m}}{ }_{\mathrm{m}}, \mathrm{F}_{\mathrm{v}} / \mathrm{F}_{\mathrm{m}}\right)$, using pulseamplitude-modulated fluorometry (MI: 9-11, SI: 8, saturation width: 0.8s; Gain up to 10 for bleached corals, Damp: 2-3; Diving-PAM, Heinz Walz GmbH, Effeltrich, Germany) were monitored on the five replicate explants per species and treatment throughout the experiment, and sampled on the last day. Data were recorded daily at noon and $30 \mathrm{~min}$ after sunset, and the values presented are means of triplicate measurements. Maximum midday excitation pressure on photosystem II $\left(Q_{m}\right)$, hereafter referred to as light pressure, was calculated as $Q_{m}$ $=1-\left[\left(\Delta F / F_{m}^{\prime}\right.\right.$ at noon $) /\left(F_{v} / F_{m}\right.$ at dusk on day 0$\left.)\right]$ (Fisher et al., 2012). $Q_{m}$ values therefore indicate the relative state of the reaction centres from fully open $\left(Q_{m}=\right.$ 0 ) to fully closed $\left(Q_{m}=1\right)$ (Maxwell \& Johnson, 2000). Symbiodinium density per unit coral surface area was calculated from haemocytometer counts $(N=6$ per explant) and determination of the coral surface area using the wax dip method (Stimson \& Kinzie, 1991). Chlorophyll extraction was performed in 1-2 mL N, Ndimethylformamide (DMF) over $48 \mathrm{~h}$ at $4^{\circ} \mathrm{C}$ in the dark. For spectrophotometric measurements, extracts were centrifuged $\left(3 \mathrm{~min}\right.$, max speed, $\left.4^{\circ} \mathrm{C}\right)$ and $200 \mu \mathrm{L}$ of the supernatant measured in triplicate at $646.8 \mathrm{~nm}, 663.8 \mathrm{~nm}$ and $750 \mathrm{~nm}$ in 96 well plates (UVStar, Greiner Bio-One, Frickenhausen, Germany). Chlorophyll a concentrations were determined after correction for optical path length $(0.555 \mathrm{~cm})$ (cf. Chapter 2, Porra et al., 1989).

\section{Genotyping}

Symbiodinium aliquots from each replicate were washed three times $(2000 \times g, 5$ $\min , 4^{\circ} \mathrm{C}$ ) and pellets genotyped by sequencing their internal transcribed spacer region (ITS2) as described previously (Logan et al., 2010). Sequences were identified using the GeoSymbio database (Franklin et al., 2012). 


\section{Biochemical analysis}

Host and Symbiodinium samples were analysed for superoxide dismutase (SOD), catalase (CAT in host), catalase peroxidase (KatG in algae) and ascorbate peroxidase (APX) activity. SOD and APX activity were determined using the protocols outlined in Chapter 2. Host CAT and algal KatG activity were determined using the same protocol for both, as outlined in Chapter 3.

Total soluble protein was determined using the improved Bradford method with BSA as the protein standard (Zor \& Selinger, 1996). After checking that protein content per cell (for Symbiodinium) and protein content per surface area (for host coral) were constant, all enzyme activities were normalized to protein content and expressed as specific activity $\left(\mathrm{U} \mathrm{mg}^{-1}\right)$. All enzyme units are defined as the amount of enzyme that catalyses one $\mu \mathrm{mol}$ of superoxide (SOD) and hydrogen peroxide (APX, CAT, KatG) per minute.

\section{Statistical analysis}

Due to the differential survival of the three coral species at each temperature, an overall analysis that included all three corals over the whole timeframe was only possible for the continuously measured variables, $F_{v} / F_{m}$ and $Q_{m}$. The overall $F_{v} / F_{m}$ and $Q_{m}$ dataset between Days 0 and 7 was analysed with a repeated measures analysis of variance (rmANOVA). $F_{v} / F_{m}$ and $Q_{m}$ results were arcsine square root and $\log \left(Q_{m}+0.2\right)$ transformed, respectively. The physiological variables, measured on the four sampling days, were tested for treatment effects over time for each coral species individually using rmANOVA. Results of Pillai's trace test or the epsilon-adjusted univariate F-test (Greenhouse-Geisser; G-G) at a confidence level of 0.05 are reported. Critical $p$-levels of reported post hoc contrasts of means between Day 0 and the day of observation were adjusted for multiple comparisons, using the Bonferroni correction. All comparisons of baseline levels (enzymes, symbiont density, $\mathrm{Chl}$ a content) in the $28^{\circ} \mathrm{C}$ treatment between host species and between Symbiodinium types on Day 0 were made by one-way ANOVA with a post hoc Tukey HSD test. Enzyme activities for three missing replicate data points $\left(A\right.$. millepora, $28^{\circ} \mathrm{C}$, Day 5 , symbiont data and Day 7 , host and symbiont data; P. damicornis, $31^{\circ} \mathrm{C}$, Day 7 , symbiont data) were estimated by extrapolating from measurements of the previous and following sampling points, to prevent the loss of the whole replicate data series for the 
rmANOVA. Statistical analysis was done using SAS JMP 10.0.0 (SAS Institute Inc., Cary, NC, USA).

\section{Results}

\section{Symbiont identity, photobiological responses and bleaching}

Symbionts were consistently identified as Symbiodinium ITS2 types C3 ( $A$. millepora), C15 (M. digitata) and C42 type 2 ( $P$. damicornis, hereafter referred to as $\mathrm{C} 42$ ). Elevated temperatures elicited significantly different responses in $F_{v} / F_{m}$ and $Q_{m}$ in all three coral species (Table 4.1, Figure 4.3A-F). Each coral showed a significant decline in $\mathrm{F}_{\mathrm{v}} / \mathrm{F}_{\mathrm{m}}$ that was greatest at the highest temperature (Figure 4.3A, Table 4.2). At $31^{\circ} \mathrm{C}$, declines in $\mathrm{F}_{\mathrm{v}} / \mathrm{F}_{\mathrm{m}}$ in $A$. millepora and $P$. damicornis over eight days were $16 \%$ and $23 \%$, respectively. While declines in $F_{v} / F_{m}$ in $M$. digitata at $31^{\circ} \mathrm{C}$ and $33^{\circ} \mathrm{C}$ were minor $(98 \%$ and $94 \%$ of control value on Day 8 , respectively), $\mathrm{F}_{\mathrm{v}} / \mathrm{F}_{\mathrm{m}}$ in $A$. millepora and $P$. damicornis dropped markedly from Day 5 at $33^{\circ} \mathrm{C}$. Total declines amounted to $51 \%$ of the control in A. millepora after 8 days and $25 \%$ in $P$. damicornis after 6 days, respectively (visual tissue loss and $80 \%$ mortality prevented any measurements in $P$. damicornis beyond Day 6 ).

Table 4.1. Overall statistical analysis of photobiological variables.

Statistical rmANOVA results for photobiological variables $F_{v} / F_{m}$ and $Q_{m}$ using the complete dataset for Acropora millepora, Montipora digitata and Pocillopora damicornis at $28^{\circ} \mathrm{C}, 31^{\circ} \mathrm{C}$ and $33^{\circ} \mathrm{C}$ over six days. Results show between-subject effects for the factors 'species' and 'temperature' and all within-subject effects. Significant effects are indicated by asterisks.

Maximum quantum yield of PSII $F_{v} / F_{m} \quad$ Light pressure $Q_{m}$

\begin{tabular}{rrr} 
Species & $F_{2,36}=11.8883, \mathrm{p}<0.0001^{*}$ & $F_{2,36}=5.4401, \mathrm{p}=0.0086^{*}$ \\
$\begin{array}{r}\text { Temperature } \\
\text { Species } \mathrm{x}\end{array}$ & $F_{2,36}=16.0726, \mathrm{p}<0.0001^{*}$ & $F_{2,36}=20.1687, \mathrm{p}<0.0001^{*}$ \\
temperature & $F_{4,36}=5.0841, \mathrm{p}=0.0024^{*}$ & $F_{4,36}=3.6711, \mathrm{p}=0.0132^{*}$ \\
\hline Time & $F_{2.0257,72.927}=13.8659, \mathrm{p}<0.0001^{*}$ & $F_{5,32}=18.3546, \mathrm{p}<0.0001^{*}$ \\
Time x species & $F_{4.0515,72.927}=1.3481, \mathrm{p}=0.2601$ & $F_{10,66}=0.9879, \mathrm{p}=0.4627$ \\
Time $\mathrm{x}$ & $F_{4.0515,72.927}=57.0159, \mathrm{p}<0.0001^{*}$ & $F_{10,66}=8.7828, \mathrm{p}<0.0001^{*}$ \\
temperature & $F_{8.103,72.927}=14.1903, \mathrm{p}<0.0001^{*}$ & $F_{20,140}=2.5295, \mathrm{p}=0.0008^{*}$ \\
$\mathrm{~T}$ x $\mathrm{x}$ species & &
\end{tabular}

Declines in $\mathrm{F}_{\mathrm{v}} / \mathrm{F}_{\mathrm{m}}$ at $31^{\circ} \mathrm{C}$ and $33^{\circ} \mathrm{C}$ were associated with significant increases in $\mathrm{Q}_{\mathrm{m}}$ in A. millepora and P. damicornis (Figure 4.3D, $\mathrm{F}$, Table 4.2). $\mathrm{Q}_{\mathrm{m}}$-values 
increased significantly from Day 6 at $31^{\circ} \mathrm{C}$ (reaching $0.42 \pm 0.06$, mean $\pm \mathrm{SE}$ on Day 9) and Day 7 at $33^{\circ} \mathrm{C}(0.75-0.8$ between Day 7 and 9) in A. millepora. A similar trend, with a one-day delay in the $31^{\circ} \mathrm{C}$ response, was observed in $P$. damicornis. Maximum $Q_{m}$, measured in the single surviving colony of $P$. damicornis at $33^{\circ} \mathrm{C}$ on Day 7 was 0.52 (Figure 4.3F). Heating to $31^{\circ} \mathrm{C}$ had no significant effect on $Q_{m}$ in $M$. digitata, and even after 9 days at $33^{\circ} \mathrm{C} Q_{m}$ in this species was lower than in the two thermally susceptible coral species (but significantly higher than in the control).

P. damicornis and A. millepora had visibly bleached by Day 7 at $33^{\circ} \mathrm{C}$. Mortality (visible as tissue sloughing) in $A$. millepora increased from $20 \%$ to $60 \%$ between Days 7 and 9 , whereas tissue loss and $80 \%$ mortality were observed as early as Day 7 in $P$. damicornis. Thus, statistical analysis for all variables at $33^{\circ} \mathrm{C}$ only considered data until Day 7 for $A$. millepora and Day 5 for $P$. damicornis (Table $4.2)$.

Prior to treatment, the three coral species exhibited significant differences in their symbiont densities $(A$. millepora $=M$. digitata $>$ P. damicornis; one-way ANOVA, $\left.F_{2,12}=12.285, \mathrm{p}=0.0012\right)$ and cell-specific chlorophyll a contents $(A$. millepora $<$ $M$. digitata $<P$. damicornis; One-way ANOVA, $\left.F_{2,11}=22.5244, p=0.0001\right)$. For $A$. millepora, declines in symbiont density, chlorophyll content per cell and unit surface area were only observed at the highest temperature, though these were not significantly different from the control over the first 7 days of treatment (Table 4.2, Figure 4.3G, Appendix Figure 7.11A, D). By Day 9, however, the two remaining $A$. millepora explants had lost $87.2 \%$ of their chlorophyll a. Symbiont densities in $P$. damicornis at $31^{\circ} \mathrm{C}$ tended to decline over the nine-day period, but were not statistically different from the control values. In contrast, symbiont densities at $33^{\circ} \mathrm{C}$ were significantly lower than in the control and $31^{\circ} \mathrm{C}$ treatment after only 5 days (Table 4.2, Appendix Figure 7.11C). Chlorophyll content per unit surface area in $P$. damicornis at $33^{\circ} \mathrm{C}$ was, however, not affected until Day 7 (Figure 4.3I), because the significant increase in chlorophyll content per symbiont cell compensated for the loss in cells (Table 4.2, Appendix Figure 7.11F). The continuing decline in symbiont density at $33^{\circ} \mathrm{C}$ led, however, to a net loss of $69.5 \%$ chlorophyll $a$ in the one remaining $P$. damicornis explant by Day 7 . No significant trends for any of the bleaching proxies were seen in Montipora 
digitata, though the cell-specific chlorophyll content tended to increase at $31^{\circ} \mathrm{C}$ from Day 7 onwards.
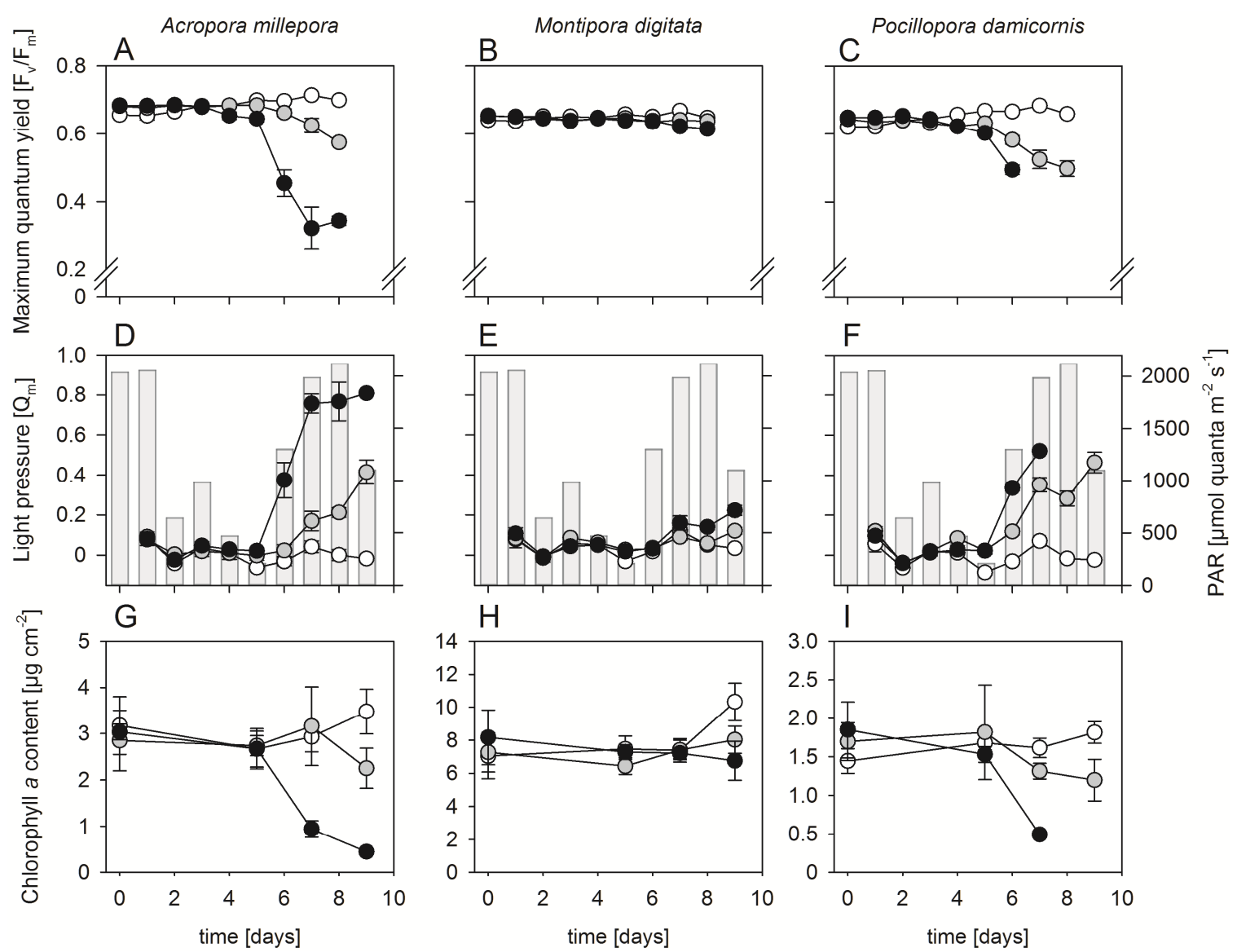

Figure 4.3. Response of photobiological variables of three coral species under thermal stress.

Overview of photobiological variables for Acropora millepora, Montipora digitata and Pocillopora damicornis at $28^{\circ} \mathrm{C}$ (white), $31^{\circ} \mathrm{C}$ (grey) and $33^{\circ} \mathrm{C}$ (black), over nine days of treatment. Shown are dark-adapted quantum yield of PSII $\left(F_{v} / F_{m}, A-C\right)$, light pressure $\left(Q_{m}, D-F\right.$, circles, left axis) and ambient photosynthetically active radiation ( $D-F$, bars, right axis), and chlorophyll a content (G-I) of the coral holobiont as interpolated scatter plots. Values are mean $\pm \mathrm{SE}, \mathrm{N}=5$, except for Days $7,8,9$ in $A$. millepora at $33^{\circ} \mathrm{C}(\mathrm{N}=$ $4,3,2)$ and Day 7 in $P$. damicornis at $33^{\circ} \mathrm{C}(\mathrm{N}=1)$.

\section{Antioxidant features}

The various coral species had significantly different baseline enzyme activities. This was mainly driven by differences in the algal symbionts, as all three coral hosts did not differ with respect to their superoxide dismutase (SOD) or catalase (CAT) activities (Figure 4.4). Significant differences between Symbiodinium types were found for SOD $\left(F_{2,12}=5.239, \mathrm{p}=0.0231\right.$; only $\mathrm{C} 3>\mathrm{C} 15$ post hoc Tukey HSD), catalase peroxidase (KatG; $F_{2,12}=27.539, p<0.0001$; $C 3=\mathrm{C} 42>\mathrm{C} 15$ ), 
and APX activity $\left(F_{2,12}=135.4368, \mathrm{p}<0.0001\right.$; C3 $>$ C42 > C15). Of note, relative to Symbiodinium C15, APX activity was $\sim 13$-fold higher in C3 and $\sim 6$-fold higher in C42.

Comparing host and symbiont showed that host tissues in all three corals expressed significantly higher baseline activities for hydrogen peroxide scavenging by CAT than did their symbionts. Activities in the hosts were higher by a factor of $\sim 5-6$ in $A$. millepora and $P$. damicornis, and $\sim 23$ in $M$. digitata (on a per unit protein basis). In contrast, baseline SOD activity was 3-5 times higher in the symbiont, than the host (Figure 4.4). However, total scavenging activity per unit surface area for both enzymes was higher in the host tissue due to the much higher protein concentration (Appendix Figure 7.12).

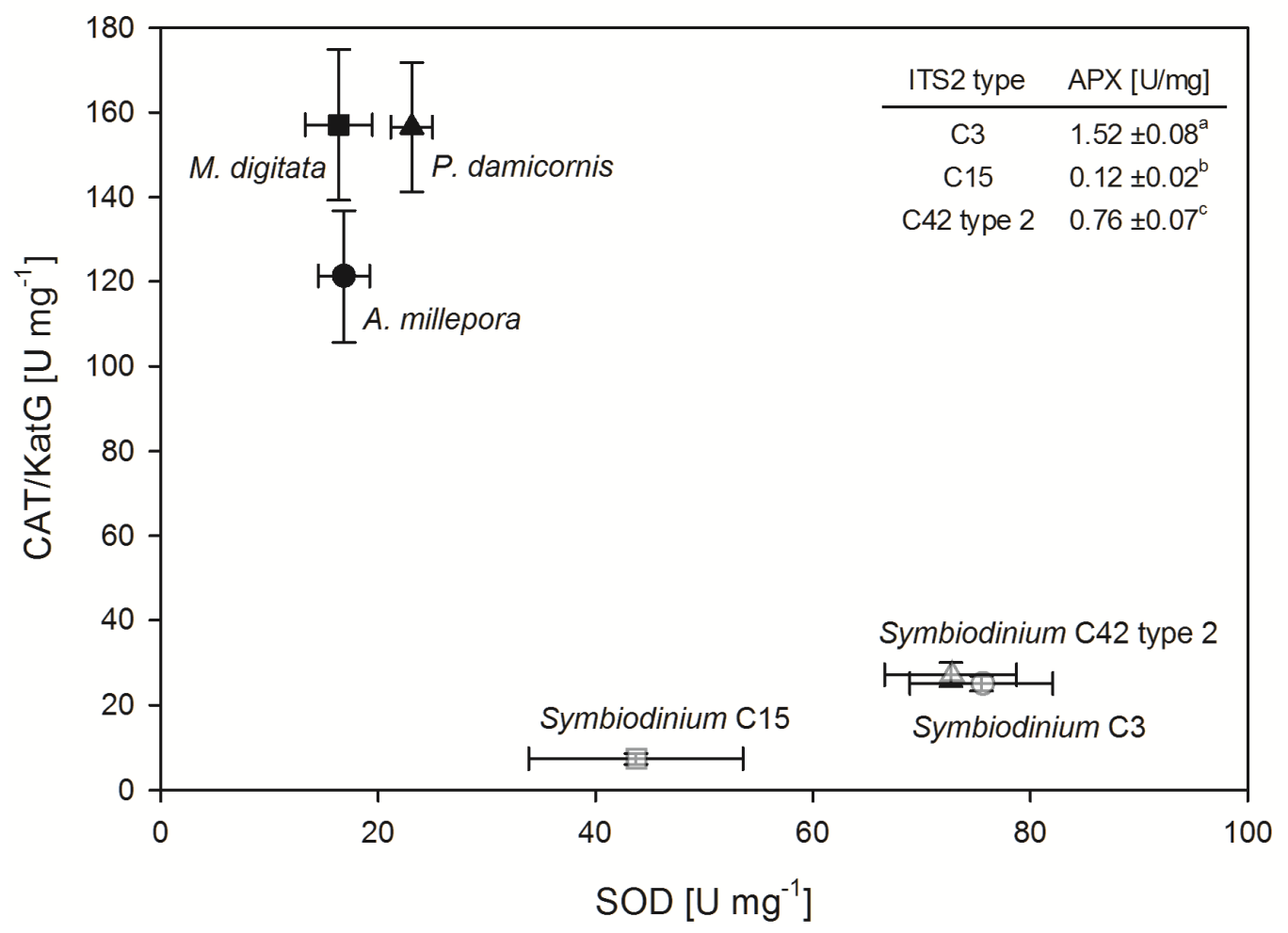

Figure 4.4. Baseline activities of enzymatic antioxidants in host and symbiont of three different coral species.

Constitutive antioxidant enzyme activities of host (black) and symbiont (white) compartments for Acropora millepora/Symbiodinium C3 (circle), Montipora digitata/Symbiodinium C15 (square) and Pocillopora damicornis/Symbiodinium C42 type 2 (triangle), in the control tank at Day 0 (mean $\pm S E, N=5$ ). Activities of superoxide dismutase (SOD), ascorbate peroxidase (APX), catalase (CAT in host) and catalase peroxidase (KatG in algae) are expressed as units per milligram of protein. Significant differences for APX activity are based on one-way ANOVA, where types not connected by the same letter are significantly different based on post hoc Tukey HSD analysis. 
Significant effects of temperature on antioxidant enzyme activities were only found for the corals $A$. millepora and $P$. damicornis. In $A$. millepora, symbiont KatG activity after five days at $31^{\circ} \mathrm{C}$ and $33^{\circ} \mathrm{C}$ was suppressed by $50-60 \%$ (Figure 4.5, Table 4.2); this difference had disappeared, however, by Day 7 for both treatments. In contrast to the situation at $31^{\circ} \mathrm{C}$, where symbiont KatG activities returned to the control level after Day 5 , activity in the two surviving colonies of $A$. millepora on Day 9 was on average twice the activity of the control treatment $\left(+105 \% ; 61.0 \pm 25.9 \mathrm{U} \mathrm{mg}^{-1}\right.$; mean $\left.\pm \mathrm{SD}\right)$. These two colonies also expressed very low symbiont APX activities (-80\%; $\left.0.23 \pm 0.01 \mathrm{U} \mathrm{mg}^{-1}\right)$ relative to the control (Figure 4.5D, G). No changes in symbiont SOD were observed in $A$. millepora. Only host CAT activity in A. millepora, but not SOD, increased at $33^{\circ} \mathrm{C}$, and a statistical effect of temperature was found when eliminating the $31^{\circ} \mathrm{C}$ dataset from the analysis ( $r$ ANOVA, $F_{\text {Time } x \text { Temperature }(2,6)}=5.7435, \mathrm{p}=0.0404$ ). Host CAT activity at $33^{\circ} \mathrm{C}$ on Day 5 was elevated by $\sim 36 \%$ in A. millepora (post hoc contrast, $p=0.0168$ ) and remained at this level until the end of the experiment (Figure 4.6D).

In $P$. damicornis, no significant temperature responses were initially found. However, one colony expressed very high symbiont SOD and APX activities at $33^{\circ} \mathrm{C}$ (corresponding to $236 \%$ and $647 \%$ of the colony average in this treatment) (Figure $4.5 \mathrm{C}, \mathrm{l}$, triangle). Corresponding activities in the host compartment for this colony were also much higher, with host SOD reaching $259 \%$ and CAT reaching $666 \%$ of the colony average in this treatment (Figure 4.6C, F, triangle). While the viability proxies, $F_{v} / F_{m}$ and $Q_{m}$, for this anomalous coral replicate showed no noteworthy differences, its chlorophyll a content per unit surface area on Day 0 was at $3.26 \mu \mathrm{g} \mathrm{cm}^{-2}$, more than twice the average value of the other four colonies (mainly due to a higher symbiont density). This colony also lost $56.9 \% \mathrm{Chl}$ a per unit surface area between Days 0 and 5 , in contrast to the average loss of $3.3 \% \pm$ $16.8 \%$ (mean $\pm S D$ ) for the remaining replicates. Excluding this anomalous replicate from the rmANOVA resulted in a significant effect of temperature on host CAT in $P$. damicornis between Days 0 and $5\left(F_{\text {TimexTemperature }(2,11)}=4.3053, \mathrm{p}\right.$ $=0.0416)$. In this case, the increase of CAT activity was $25-28 \%$ higher at $33^{\circ} \mathrm{C}$ than at $28^{\circ} \mathrm{C}$ and $31^{\circ} \mathrm{C}$ (post hoc contrast: $28^{\circ} \mathrm{C}$ vs. $33^{\circ} \mathrm{C}, \mathrm{p}=0.0242 ; 31^{\circ} \mathrm{C}$ vs. 
$\left.33^{\circ} \mathrm{C} p=0.0264 ; \mathrm{N}=4\right)$. Temperature had, however, no effect on any of the other antioxidants.
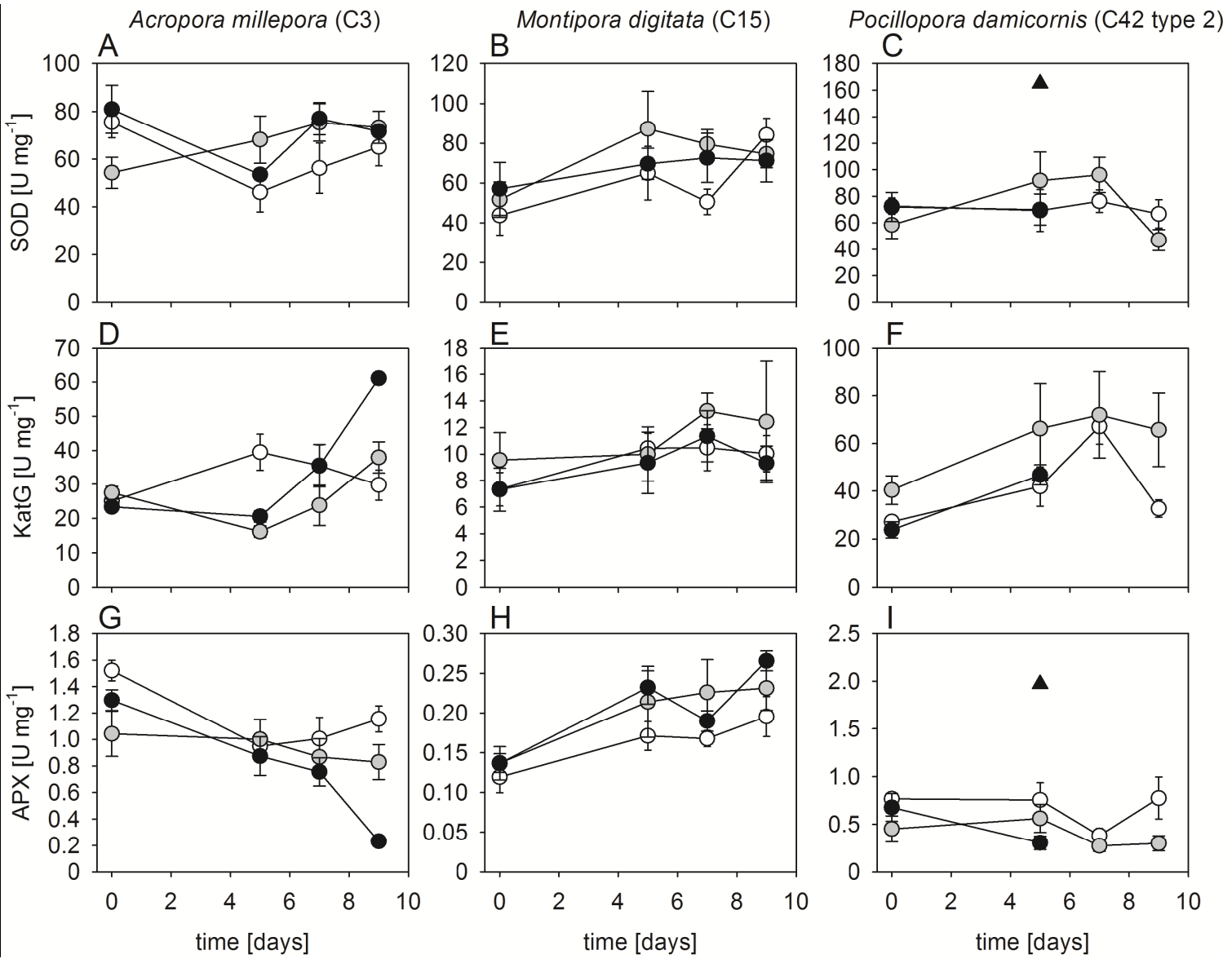

Figure 4.5. Symbiont antioxidant activity.

Symbiont antioxidant activity for superoxide dismutase (SOD, A-C) catalase peroxidase (KatG, D-F) and ascorbate peroxidase (APX, G-I) for the corals Acropora millepora, Montipora digitata and Pocillopora damicornis at $28^{\circ} \mathrm{C}$ (white), $31^{\circ} \mathrm{C}$ (grey) and $33^{\circ} \mathrm{C}$ (black), over nine days of exposure shown as interpolated scatter plots. All enzyme activities are expressed as units per milligram of protein. Values are mean $\pm \mathrm{SE}, \mathrm{N}=5$, except for Day 9 in $A$. millepora at $33^{\circ} \mathrm{C}(\mathrm{N}=2)$ and Day 5 in $P$. damicornis at $33^{\circ} \mathrm{C}$, where $\mathrm{N}=4$. The anomalous replicate of $P$. damicornis at $33^{\circ} \mathrm{C}$ on Day 5 (see text) is indicated by a triangle. Note different scaling. 

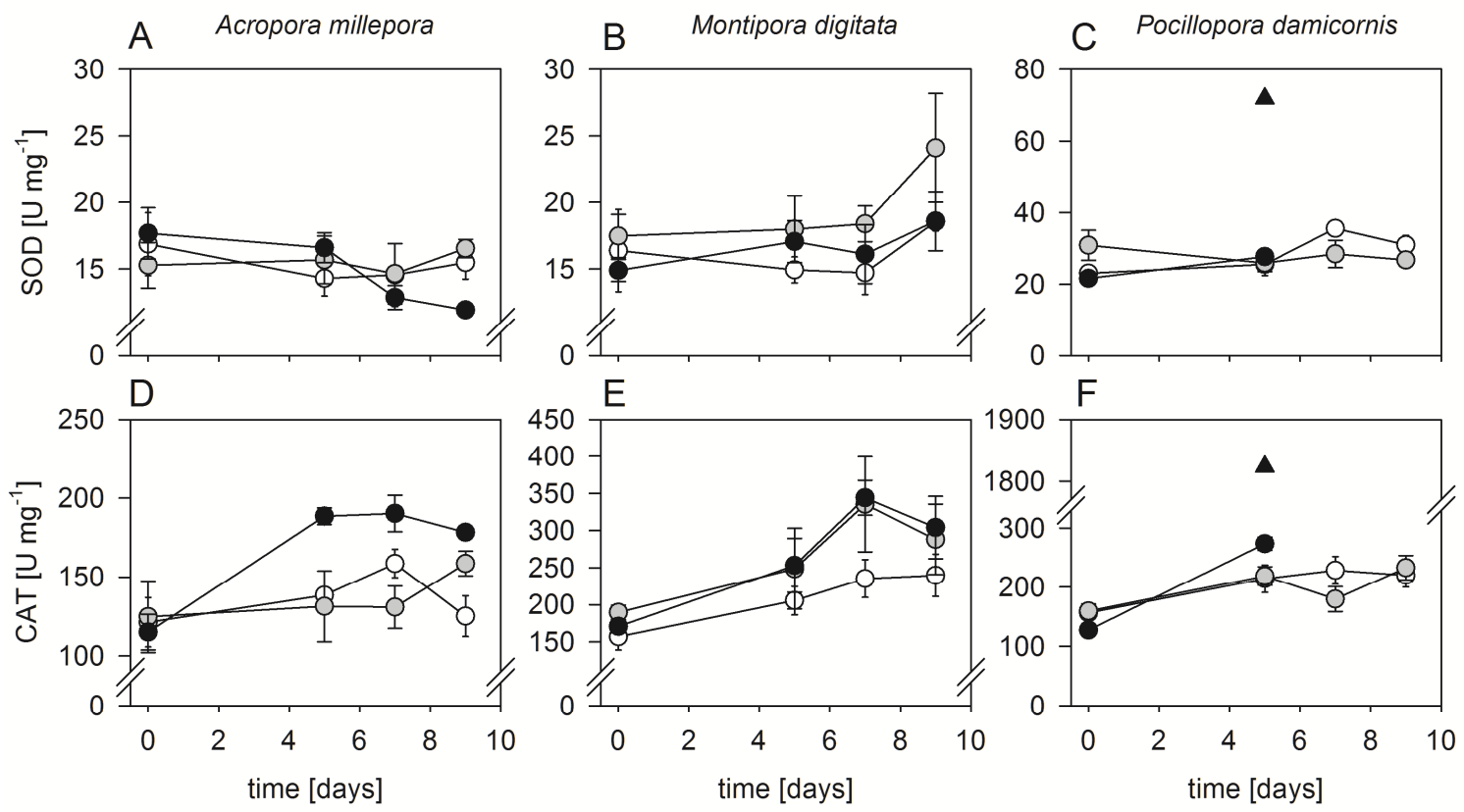

Figure 4.6. Host antioxidant activity.

Host antioxidant activity for superoxide dismutase (SOD, A-C) and catalase (CAT, D-F) for the corals Acropora millepora, Montipora digitata and Pocillopora damicornis at $28^{\circ} \mathrm{C}$ (white), $31^{\circ} \mathrm{C}$ (grey) and $33^{\circ} \mathrm{C}$ (black), over nine days of exposure shown as interpolated scatter plots. All enzyme activities are expressed as units per milligram of protein. Values are mean $\pm \mathrm{SE}, \mathrm{N}=5$, except for Day 9 in $A$. millepora at $33^{\circ} \mathrm{C}(\mathrm{N}=2)$ and Day 5 in $P$. damicornis at $33^{\circ} \mathrm{C}$ where $\mathrm{N}=4$. The anomalous replicate of $P$. damicornis at $33^{\circ} \mathrm{C}$ on Day 5 (see text) is indicated by a triangle. 
Table 4.2. Statistical analysis of physiological variables for each coral.

Statistical rmANOVA results for photobiological and antioxidant variables in Acropora millepora, Montipora digitata and Pocillopora damicornis at $28^{\circ} \mathrm{C}, 31^{\circ} \mathrm{C}$ and $33^{\circ} \mathrm{C}$. Results show all within-subject effects. Due to differential survival of corals, time periods for analysis differ between corals. Significant effects at $p<0.05$ are indicated by an asterisk.

\begin{tabular}{|c|c|c|c|c|}
\hline Variable & Time period & Time & Time x Temperature & $\begin{array}{c}\text { Time } x \text { Temperature } \\
\left(28^{\circ} \mathrm{C} \text { vs } 31^{\circ} \mathrm{C} \text { over } 9 \text { days }\right)\end{array}$ \\
\hline \multicolumn{5}{|l|}{ ACROPORA MILLEPORA } \\
\hline$\overline{F_{v} / F_{m}}$ & 7 Days & $F_{1.2007,13.208}=2.3953, p=0.1428$ & $\mathrm{~F}_{2.4015,13.208}=30.6175, \mathrm{p}<0.0001^{\star}$ & $\mathrm{F}_{2.8033,22.426}=31.7197, \mathrm{p}<0.0001^{*}$ \\
\hline $\mathrm{Q}_{\mathrm{m}}$ & 7 Days & $F_{6,6}=4.7336, p=0.0401^{*}$ & $F_{12,14}=3.3113, p=0.0180^{*}$ & $F_{8,1}=167.1899, p=0.0597$ \\
\hline Symbiont density [cells $/ \mathrm{cm}^{-2}$ ] & 7 Days & $F_{2,10}=0.3954, p=0.6835$ & $F_{4,22}=2.9154, p=0.0658$ & $F_{3,6}=2.8092, p=0.1303$ \\
\hline Symbiont Chl a content $\left[\mathrm{pg} \mathrm{cell}{ }^{-1}\right]$ & 7 Days & $F_{2,10}=0.1575, p=0.8564$ & $F_{4,22}=1.7673, p=0.1714$ & $F_{3,6}=1.9954, p=0.2162$ \\
\hline Holobiont Chl a content $\left[\mu \mathrm{g} \mathrm{cm}^{-2}\right]$ & 7 Days & $F_{2,10}=0.3772, p=0.6951$ & $F_{4,22}=1.7858, p=0.1677$ & $F_{3,6}=0.9296, p=0.4820$ \\
\hline Host SOD $\left[\mathrm{U} \mathrm{mg}^{-1}\right]$ & 7 Days & $F_{2,10}=0.5379, p=0.6000$ & $F_{4,22}=0.6165, p=0.6554$ & $F_{3,6}=0.3024, p=0.8231$ \\
\hline Host CAT [U mg $\left.{ }^{-1}\right]$ & 7 Days & $F_{2,10}=2.5366, p=0.1285$ & $F_{4,22}=1.9430, p=0.1389$ & $F_{3,6}=1.9825, p=0.2181$ \\
\hline Symbiont SOD [U mg-1] & 7 Days & $F_{2,9}=2.9042, p=0.1064$ & $F_{4,18}=1.7453, p=0.1797$ & $F_{3,6}=1.5538, p=0.2952$ \\
\hline Symbiont APX[U mg-1] & 7 Days & $F_{2,9}=5.3255, p=0.0298^{*}$ & $F_{4,18}=1.0789, p=0.3933$ & $F_{3,6}=4.1277, p=0.0660$ \\
\hline Symbiont KatG [U mg-1] & 7 Days & $F_{2,9}=8.3813, p=0.0088^{*}$ & $F_{4,20}=3.6872, p=0.0209^{\star}$ & $F_{3,6}=6.9972, p=0.0219^{*}$ \\
\hline \multicolumn{5}{|l|}{ MONTIPORA DIGITATA } \\
\hline $\mathrm{F}_{\mathrm{v}} / \mathrm{F}_{\mathrm{m}}$ & 8 Days & $F_{8,5}=1.9110, p=0.2465$ & $F_{16,12}=2.4292, p=0.0627$ & $F_{8,1}=0.4557, p=0.8232$ \\
\hline $\mathrm{Q}_{\mathrm{m}}$ & 9 Days & $F_{8,5}=33.4402, p=0.0006^{*}$ & $F_{16,12}=5.2715, p=0.0029^{\star}$ & $F_{4.0744,32.595}=2.2010, p=0.0897$ \\
\hline Symbiont density [cells/cm ${ }^{-2}$ ] & 9 Days & $F_{3,5}=3.5693, p=0.1023$ & $F_{6,12}=0.9576, p=0.4919$ & $F_{3,5}=2.4012, p=0.1838$ \\
\hline Symbiont Chl a content $\left[\mathrm{pg}\right.$ cell $\left.^{-1}\right]$ & 9 Days & $F_{3,8}=1.1589, p=0.3835$ & $F_{6,18}=2.6049, p=0.0537$ & $F_{3,5}=1.9000, p=0.2475$ \\
\hline Holobiont Chl a content $\left[\mu \mathrm{g} \mathrm{cm}^{-2}\right]$ & 9 Days & $F_{1.4677,11.742}=0.4892, p=0.1191$ & $F_{2.9355,11.742}=0.6090, p=0.6189$ & $F_{1.5112,10.578}=0.9279, p=0.3988$ \\
\hline Host SOD [U mg-1] & 9 Days & $F_{3,10}=0.5671, p=0.6491$ & $F_{6,22}=0.2435, p=0.9569$ & $F_{3,6}=0.1681, p=0.9141$ \\
\hline Host CAT [U mg $\left.{ }^{-1}\right]$ & 9 Days & $F_{3,10}=1.9060, p=0.1925$ & $F_{6,22}=0.6868, p=0.6623$ & $F_{3,6}=0.5135, p=0.6878$ \\
\hline Symbiont SOD [U mg $\left.{ }^{-1}\right]$ & 9 Days & $F_{3,10}=8.5437, p=0.0041^{*}$ & $F_{6,22}=1.5348, p=0.2143$ & $F_{3,6}=2.1044, p=0.2011$ \\
\hline Symbiont APX[U mg-1] & 9 Days & $F_{1.6254,19.505}=2.0156, p=0.1656$ & $F_{3.2508,19.505}=0.6380, p=0.6114$ & $F_{1.4704,11.764}=0.2258, p=0.7344$ \\
\hline Symbiont KatG [U mg $\left.{ }^{-1}\right]$ & 9 Days & $F_{2.0043,24.052}=0.6121, p=0.5508$ & $F_{4.0086,24.052}=0.1774, p=0.9481$ & $F_{3,6}=0.2038, p=0.8902$ \\
\hline
\end{tabular}


Table 4.2 [continued]

\begin{tabular}{|c|c|c|c|c|}
\hline Variable & Time period & Time & Time $\mathrm{x}$ Temperature & $\begin{array}{c}\text { Time } \times \text { Temperature } \\
\left(28^{\circ} \mathrm{C} \text { vs } 31^{\circ} \mathrm{C} \text { over } 9 \text { days }\right)\end{array}$ \\
\hline \multicolumn{5}{|l|}{ POCILLOPORA DAMICORNIS } \\
\hline $\bar{F}_{\mathrm{v}} / \mathrm{F}_{\mathrm{m}}$ & 6 Days & $F_{1.8934,22.72}=11.4899, p=0.0004^{*}$ & $F_{3.7867,22.72}=38.7964, p<0.0001^{*}$ & $F_{1.7139,13.711}=29.5001, p<0.0001^{*}$ \\
\hline $\mathrm{Q}_{\mathrm{m}}$ & 6 Days & $F_{2.4921,29.905}=14.8441, p<0.0001^{*}$ & $\mathrm{~F}_{4.9842,29.905}=9.3233, \mathrm{p}<0.0001^{*}$ & $F_{2.7357,21.886}=14.8779, p<0.0001^{*}$ \\
\hline Symbiont density [cells $/ \mathrm{cm}^{-2}$ ] & 5 Days & $F_{1,11}=4.2109, p=0.0647$ & $F_{2,11}=10.7232, p=0.0026^{\star}$ & $F_{3,6}=2.3475, p=0.1720$ \\
\hline Symbiont Chl a content $\left[\mathrm{pg}\right.$ cell $\left.{ }^{-1}\right]$ & 5 Days & $F_{1,10}=0.8488, p=0.3786$ & $F_{2,10}=6.3760, p=0.0164^{*}$ & $F_{3,5}=0.5787, p=0.6539$ \\
\hline Holobiont $\mathrm{Chl}$ a content $\left[\mu \mathrm{g} \mathrm{cm}^{-2}\right]$ & 5 Days & $F_{1,10}=0.1520, p=0.7048$ & $F_{2,10}=0.3854, p=0.6899$ & $F_{3,5}=1.7419, p=0.2739$ \\
\hline Host SOD [U mg $\left.{ }^{-1}\right]$ & 5 Days & $F_{1,12}=0.1699, p=0.6875$ & $F_{2,12}=2.7969, p=0.1007$ & $F_{3,6}=1.7267, p=0.2604$ \\
\hline Host CAT [U mg $\left.{ }^{-1}\right]$ & 5 Days & $F_{1,12}=0.1014, p=0.7556$ & $F_{2,12}=1.5910, p=0.2438$ & $F_{3,6}=1.7714, p=0.2523$ \\
\hline Symbiont SOD [U mg $\left.{ }^{-1}\right]$ & 5 Days & $F_{1,12}=0.0191, p=0.8923$ & $F_{2,12}=0.5670, p=0.5817$ & $F_{3,6}=1.0130, p=0.4499$ \\
\hline Symbiont APX $\left[\mathrm{U} \mathrm{mg}^{-1}\right]$ & 5 Days & $F_{1,12}=0.0024, p=0.9616$ & $F_{2,12}=0.1061, p=0.9002$ & $F_{1.6358,13.087}=0.6794, p=0.4955$ \\
\hline Symbiont KatG $\left[\mathrm{U} \mathrm{mg}{ }^{-1}\right]$ & 5 Days & $F_{1,12}=1.5261, p=0.2403$ & $F_{2,12}=0.2321, p=0.7963$ & $F_{3,6}=1.7605, p=0.2543$ \\
\hline
\end{tabular}




\section{Discussion}

This study investigated the differential bleaching response of three common IndoPacific coral species from the Heron Island reef flat during a simulated heating event, exposing them to $3-5^{\circ} \mathrm{C}$ above ambient temperatures. While bleaching and photobiological stress was evident in two susceptible coral species at the highest temperature, no significant up-regulation of antioxidant defences in their symbionts was observed. In contrast, increased scavenging of hydrogen peroxide in the host compartment at this temperature preceded algal dysfunction and overall bleaching in these corals.

\section{Photophysiological stress at high temperatures}

A visual bleaching response - manifested as paling in Acropora millepora, and paling and tissue sloughing in Pocillopora damicornis - was not apparent until after seven days of exposure to $33^{\circ} \mathrm{C}$; there was also a significant decline in chlorophyll content per unit surface area between Days 5 and 7 at this temperature. Substantial changes in the photobiological parameters $F_{v} / F_{m}$ and $Q_{m}$ coincided with these bleaching responses, which were consistent with previous findings (Hoegh-Guldberg \& Jones, 1999, Warner et al., 1999, FerrierPagès et al., 2007). High values of $Q_{m}$ show that under full daylight some of the PS II reaction centres were inactivated, indicating dynamic or irreversible photoinhibition (Maxwell \& Johnson, 2000). However, for advanced bleaching states, increases in $Q_{m}$ might also be related to a brighter light environment for the remaining symbionts, as a result of the decrease in light absorption and increase in light scattering by the coral skeleton (Enríquez et al., 2005). In contrast, the lack of bleaching in both A. millepora and P. damicornis at $31^{\circ} \mathrm{C}$, despite moderate reductions in $F_{v} / F_{m}$ and increases in $Q_{m}$, suggests that the corals were within an acceptable range for their protective or photoacclimatory mechanisms, e.g., thermal dissipation of light energy (Gorbunov et al., 2001, Fisher et al., 2012). In contrast to A. millepora and P. damicornis, Montipora digitata was thermally robust, confirming previous findings for this species (Fisher et al., 2012). 


\section{Oxidative stress in host and symbiont as a temperature response}

In the current study, the photophysiological responses of $A$. millepora and $P$. damicornis indicated an adverse effect of high temperature. However, this was not mirrored by enzymatic responses that would likely accompany oxidative stress. For example, the major enzymatic antioxidants SOD, APX and KatG increase their activities in thermally-challenged Symbiodinium in culture (Chapter 2, Lesser, 1996, McGinty et al., 2012), but this was, with the exception of one single $P$. damicornis colony, not the case in the two bleaching corals here. The only suggestion of oxidative stress in both thermally-susceptible coral species was elevated host CAT activity, indicating an increased turnover of hydrogen peroxide via this enzyme. As no changes in host SOD were observed, two possibilities for these elevated levels of CAT exist: (1) host catalase may have responded to leakage of hydrogen peroxide from the symbiont as suggested by the Oxidative Theory of Coral Bleaching; (2) increased CAT might result from elevated metabolic activity in the host. Measurements of ROS production in the host and symbionts would have helped to resolve this issue.

Previous studies investigating the effects of thermal stress on Symbiodinium when in hospite have found enhanced symbiont SOD activity (Anemonia viridis; Richier et al., 2005, Galaxea fascicularis; Higuchi et al., 2008, Higuchi et al., 2012), consistent with photophysiological stress and the formation of superoxide via the Mehler reaction (Asada, 1984). Host SOD, in comparison, may (Orbicella [Syn. Montastraea] faveolata; Downs et al., 2000; Stylophora pistillata; Yakovleva et al., 2004) or may not (Platygyra ryukyuensis; Yakovleva et al., 2004; A. viridis; Richier et al., 2005) respond to elevated temperature. Similarly, increased host tissue levels of ROS, in the form of superoxide or hydroxyl radicals in response to elevated temperature can be either present (Aiptasia pulchella; Nii \& Muscatine, 1997) or absent (G. fascicularis; Higuchi et al., 2010). Clearly, cnidarian species differ significantly in their cellular physiology and the strategies employed to mitigate oxidative stress. But, consistent observations in support of the Oxidative Theory of Bleaching have largely arisen from studies that manipulated irradiance (including ultraviolet radiation) rather than temperature, or investigated the effects of bathymetric gradients (Dykens \& Shick, 1984, Lesser \& Shick, 1989b, Lesser \& Shick, 1989a, Dykens et al., 1992, Shick et al., 1995, Downs et al., 2002, 
Lesser \& Farrell, 2004, Levy et al., 2006, Kuguru et al., 2010). It should also be noted that increased antioxidant activity in corals can arise in response to pollution, aerial exposure or seasonal environmental variability, without necessarily eliciting a bleaching response (Liñán-Cabello et al., 2010, Ramos et al., 2011, Teixeira et al., 2013).

When considering why no up-regulation of antioxidant defences was seen in the hosts and symbionts in the current study, it is worth considering the rate of experimental heating. Heating rate profoundly affects the physiological response of the coral holobiont (Middlebrook et al., 2010) and the $1^{\circ} \mathrm{C}$ per day ramping used in the current study is considerably slower than in the previously mentioned studies of antioxidant defences under thermal stress; these have applied heating rates ranging from instantaneous to $4^{\circ} \mathrm{C}$ per hour (Lesser, 1996, Yakovleva et al., 2004, Richier et al., 2005, Higuchi et al., 2008, Yakovleva et al., 2009, Higuchi et al., 2012; T. Higuchi and I. Yakovleva, personal communication). Not only are such rapid temperature increases not representative of bleaching conditions in the field, but they limit the capacity to measure and distinguish between shortand long-term responses. For example, the corals Acropora intermedia and Montipora digitata from the South China Sea lost over $90 \%$ of their symbionts within 48 hours after an experimental $4^{\circ} \mathrm{C}$ per hour temperature ramp, from $25^{\circ} \mathrm{C}$ to $33^{\circ} \mathrm{C}$ (Imbs \& Yakovleva, 2012). Furthermore, it is unknown whether or how heating rate affects the mechanism by which bleaching occurs, i.e., the spectrum of responses from symbiont in situ degradation to host cell apoptosis and necrosis (reviewed in Weis, 2008). However, while acknowledging the limitations of fast heating experiments, slower scenarios with longer sampling intervals as applied in this study also bear the risk of missing important information. For example, potential antioxidant responses between Days 0 and 5 in this experiment could have been missed and not considered in the physiological interpretation. However, such changes would also have been independent of maximum quantum yields, which remained constant in all corals.

Variation in experimental settings and thermal scenarios is important if we are to correctly interpret coral bleaching experiments, and the contrast between shortand long-term responses is becoming increasingly evident as transcriptomic 
studies allow a broader view of the dynamic molecular responses of both partners (Leggat et al., 2011, Meyer et al., 2011).

\section{ROS and coral bleaching - the role of hydrogen peroxide}

This study showed that catalase activity per unit protein is five to twenty-three times higher in the host than in the symbiont, as also reported in several previous studies (Yakovleva et al., 2004, Levy et al., 2006, Higuchi et al., 2008). Assuming that the primary site of ROS production under light and temperature stress is the symbiont chloroplast, ROS have to pass through four membranes (i.e., three chloroplastic membranes and one plasma membrane) and the cell wall to leave the symbiont, where they potentially encounter additional symbiont-derived membranes and the final host-derived symbiosome membrane (Wakefield \& Kempf, 2001). The high number of enclosed compartments supports the suggestion that hydrogen peroxide is the primary form in which reactive oxygen leaves the symbiont, as it has, in comparison to other ROS, a comparably long lifetime, a large diffusion radius, and no net charge (Winterbourn, 2008). Potential leakage of hydrogen peroxide (but also extracellular generation of ROS) from Symbiodinium and other dinoflagellates has been shown experimentally (Palenik \& Morel, 1990, Sandeman, 2006, Suggett et al., 2008, Saragosti et al., 2010). However, estimates of baseline scavenging capacities from this study suggest that extraordinarily high levels of hydrogen peroxide would need to leak from the symbionts in order to challenge or overwhelm the host's antioxidant defences (not considering the capacity of other enzymatic and non-enzymatic systems). In fact, the scavenging capacity of the symbionts is estimated to be less than that of the host (on a per unit protein basis), suggesting that host hydrogen peroxide might pose a greater oxidative risk for the symbionts rather than the other way round. Thermal stress-induced degradation of cnidarian host mitochondria that potentially limits the capacity to scavenge mitochondrial ROS effectively in the host tissue has been shown recently (Dunn et al., 2012). The potential importance of host-produced hydrogen peroxide is highlighted in the current study, where host catalase activity in $A$. millepora at $33^{\circ} \mathrm{C}$ remained high until the end of the experiment, despite photosynthetic dysfunction that might be expected to limit ROS synthesis as a result of lower rates of oxygen evolution (Nii \& Muscatine, 1997, Richier et al., 2005). The presence of a high hydrogen peroxide 
concentration of host origin could also explain the KatG increase and APX inhibition in the symbionts at this temperature, since neither was related to changes in symbiont SOD levels.

It is clear that we still have much to learn about the fluxes of ROS between host and symbiont. Studies on oxidative damage to the symbiosome membrane that forms the interface between the host and symbiont would considerably improve our understanding about these fluxes. Moreover, we still have much to learn about the relative sensitivity of the two partners to oxidative stress and how this determines the thermal sensitivity of particular host-symbiont combinations. For example, while differences in CAT/KatG and SOD activities between Symbiodinium types and host species were apparent here, it is not yet possible to confirm or deny whether antioxidant defences determine thermal tolerance. Of particular note, the thermally tolerant M. digitata harboured Symbiodinium C15, which contained lesser enzymatic antioxidant defences than did the more thermally sensitive Symbiodinium types studied here. This highlights the importance of mechanisms that prevent the generation of ROS upstream of antioxidant defences as well as the potentially important regulatory role of the host.

\section{Antioxidants and bleaching in corals - the role of the host}

This study indicates that an antioxidant response is mainly mounted in the host compartment of two thermally-susceptible coral species at least two days before visible bleaching and photosynthetic stress are observed in their algal symbionts. The study of Hawkins et al. (2014), which was run in tandem with the current study, adds to this observation by illustrating a transient up-regulation of host caspase-like enzyme activity - indicative of apoptotic-like cell death - in $A$. millepora at $33^{\circ} \mathrm{C}$, that coincided with higher catalase-mediated hydrogen peroxide turnover in the host (this study). A correlative relationship between induction of nitric oxide synthesis and apoptosis under thermal stress in the host has been suggested (Hawkins et al., 2013, Hawkins et al., 2014), and the patterns seen in $A$. millepora highlight the role that ROS might play in the activation of apoptotic-like pathways. However, in $P$. damicornis at $31^{\circ} \mathrm{C}$, a similar response with respect to host caspases (Hawkins et al., 2014) did not coincide with an upregulation in enzymatic antioxidants (this study). The relationship 
between ROS and apoptosis in the coral host therefore appears to be speciesspecific.

In summary, this study shows that, under the thermal scenario tested here, no change in antioxidant defences occurs in the dinoflagellate symbiont, while host defences in thermally susceptible corals increase in the form of enhanced scavenging of hydrogen peroxide, and precede bleaching. These findings raise questions about whether the symbiont is the primary source of ROS in the bleaching process and highlight the potential role of the host in thermal bleaching. 


\section{General discussion}

One of the most ecologically significant symbioses formed by dinoflagellates of the genus Symbiodinium is that with reef-building corals. This genus is genetically diverse, geographically widespread and associates not only with corals, but also with a variety of other taxa. To what extent different Symbiodinium genotypes vary in their sensitivity to stress and how they influence the thermal threshold of reef-building corals are two fundamental questions in coral biology. This thesis investigated one particular aspect of the stress physiology of Symbiodinium and corals: The role of antioxidant defences under thermal stress. More specifically, the thesis focused on how fundamental mechanisms to remove potentially harmful reaction oxygen species (ROS) differ between Symbiodinium types and whether they contribute to differential bleaching in the intact symbiosis. Within the scope of this thesis, I was able to test a number of hypotheses (Chapter 1, page 22) and the key findings show that:

(1) Scavenging rates of reactive oxygen by particular enzymatic antioxidants differ considerably between Symbiodinium types under the same environmental conditions in vitro and in hospite.

(2) Symbiodinium type differences are smaller for enzymes involved in the first line of defence (i.e. those that scavenging superoxide), than in downstream systems (i.e. those involved, either directly or indirectly, in hydrogen peroxide scavenging).

(3) While the activity of primary enzymatic antioxidants in response to thermal exposure is similar between sensitive and tolerant Symbiodinium types in culture, sensitive types consistently fail to maintain redox homeostasis in the glutathione system.

(4) Some antioxidant genes in Symbiodinium have likely been acquired through horizontal gene transfer from other taxa. 
(5) Superoxide dismutase (SOD) isoforms are highly conserved across cladal and sub-cladal boundaries.

(6) Protein evolution of two peroxidases reflects the genetic radiation of the genus Symbiodinium

(7) Thermal sensitivity of the coral holobiont is primarily reflected in changes of ROS scavenging in the host compartment rather than the symbiont under the experimental settings used here.

(8) Enzymatic scavenging capacity for hydrogen peroxide is considerably higher in the host than the symbiont.

This thesis has identified the antioxidant network as an important component in explaining differential thermal susceptibility in Symbiodinium types. Through the analysis of network responses, and characteristics of individual enzymatic components of this network, it was possible to link the diversity of Symbiodinium to type specific antioxidant profiles and mechanisms that are relevant for the thermal response in culture. Based on the field study conducted here, these properties, while still relevant for specific types, might, however, be more or less responsive under different thermal scenarios in hospite. Instead, host responses, including antioxidant and apoptotic processes may play a very important role in the initiation of coral bleaching.

In the following sections, I review the antioxidant physiology of different Symbiodinium types and discuss some aspects that might have promoted the development of this. Considering the varying experimental approaches used in previous studies on coral bleaching, I will discuss the potentially important role of heating rates on the differential temporal response of host and symbiont and discuss some antioxidant host features in the context of the Oxidative Theory of Coral Bleaching. 


\section{How Symbiodinium genotypes differ in their antioxidant physiology}

Recognizing the diversity of the genus Symbiodinium requires the use of molecular genetic markers. Nine major clades have been established, and using faster evolving markers, hundreds of genetically different types within these clades have been consistently identified (Rowan \& Powers, 1991b, Rowan \& Powers, 1991a, LaJeunesse, 2001, LaJeunesse, 2005, Sampayo et al., 2009, Pochon \& Gates, 2010). More importantly, this genetic diversity extends to the protein level, and early experiments on 17 different Symbiodinium cultures showed that electrophoretic banding patterns of proteins are an intrinsic property of these Symbiodinium types and are consistently present, independent of host association and geographical origin (Schoenberg \& Trench, 1980a). Proteins are the functional level at which DNA mutations can act and, in more recent studies, transcriptome analysis of different Symbiodinium types has shown that genetic divergence had a considerable impact on a number of orthologous proteins, of which some are relevant for processes of thermal tolerance in Symbiodinium (Voolstra et al., 2009, Bayer et al., 2012, Ladner et al., 2012). In recognition of the important role of reactive oxygen in the coral bleaching cascade (reviewed in Lesser, 2011), the goal of this thesis was to contrast the antioxidant system in different Symbiodinium types by focusing on two aspects: the differential activity of parts of the network under thermal stress (Chapter 2), and the extent of structural variability of important enzymatic elements of this network (Chapter 3 ) as a result of the genetic radiation of the genus.

Detoxification of superoxide via SOD and ascorbate peroxidase (APX) was a common feature in all investigated Symbiodinium types in culture at $33^{\circ} \mathrm{C}$. The exception was type E1, where APX activity was not correlated with SOD, highlighting the importance of alternative pathways of hydrogen peroxide scavenging in this particular type. It was not possible to distinguish sensitive (type B1, C1 and E) and tolerant types (F1) based solely on the response of the SODAPX-pathway, despite declining photosynthetic function and cell death in the former. Features of the antioxidant response that were directly connected to survival at high temperatures were in fact only present in the glutathione system, 
particularly with respect to its redox state. It was also this system that illustrated the mechanisms that accompanied the transition from acclimation/survival at sublethal temperatures to oxidative stress/death at $33^{\circ} \mathrm{C}$ in the most susceptible type, Symbiodinium B1. This is the first study to show that glutathione redox homeostasis is compromised in thermally susceptible Symbiodinium types. Further research, combining different light-temperature scenarios that include a larger number of Symbiodinium types, is needed to establish whether the glutathione redox state and its regulation at sub-lethal temperatures is indeed a suitable indicator of thermal sensitivity. While the focus of this study was primarily on the effects of temperature, future studies that investigate the interaction of higher light and temperature settings are required. Since light it is an important driver for processes that generate ROS in photosynthetic organisms, the rate and scale of antioxidant upregulation would have likely been stronger for higher irradiances.

From a mechanistic point of view, thermal tolerance at $33^{\circ} \mathrm{C}$ in Symbiodinium $\mathrm{F} 1$ is achieved through a proportionally higher up-regulation of the cellular SOD-APX pathway and stability of the glutathione redox state (as a combination of higher glutathione synthesis and recycling). The glutathione redox state in the most thermally susceptible type, B1, on the other hand, can only be maintained up to $29^{\circ} \mathrm{C}$. The collapse of regulating processes, such as recycling through glutathione reductase, leads to a redox imbalance and cellular damage. Moreover, the removal of end products of oxidative damage via enzymes such as glutathione S-transferase further exacerbates the cellular redox homeostasis. Based on the experiments performed, these processes seem to be fundamental in explaining the molecular basis for low and high thermal thresholds in Symbiodinium at low light levels (Figure 5.1). 


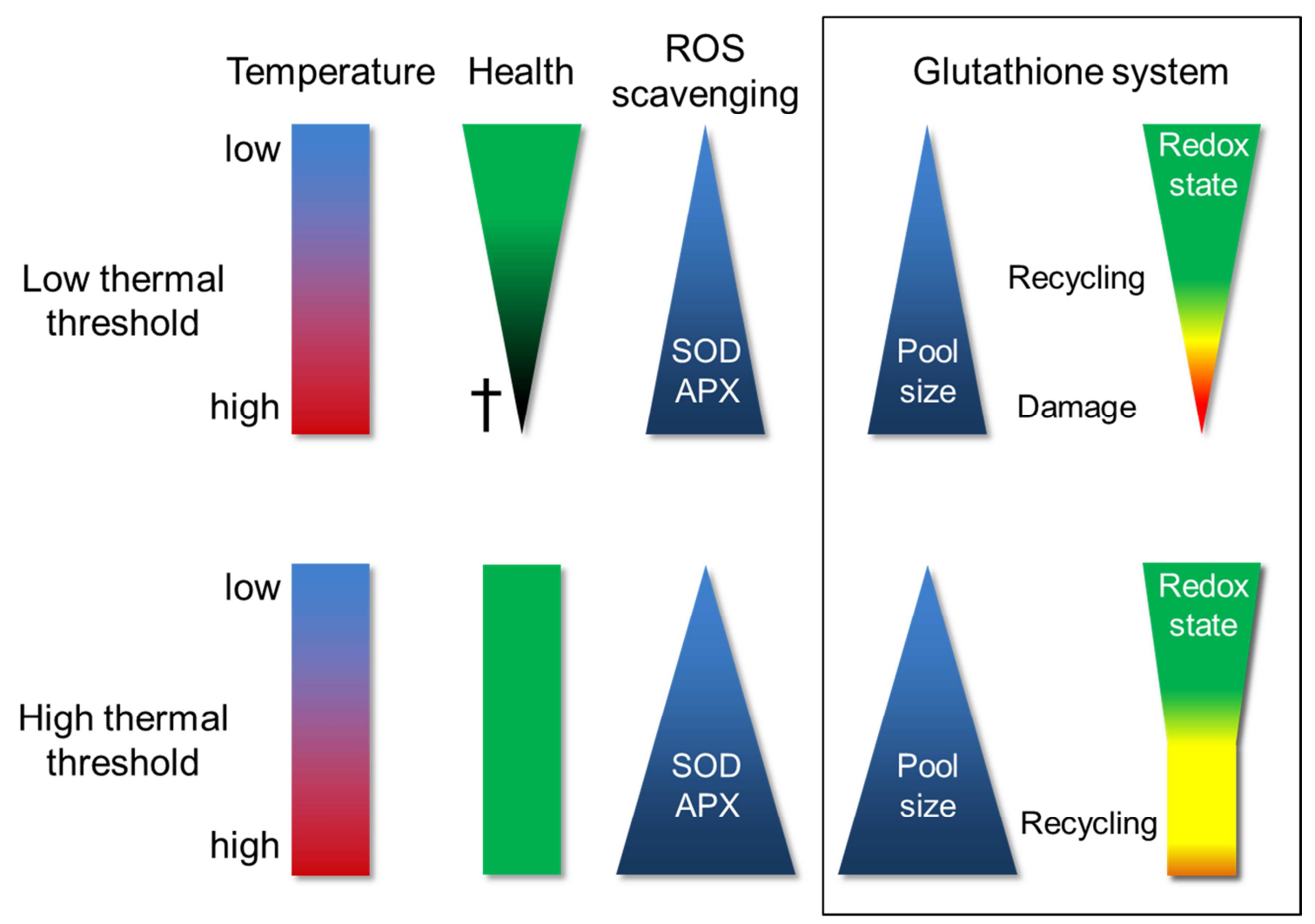

Figure 5.1. Key antioxidant processes that define thermal thresholds in Symbiodinium at low light intensities.

Simplified overview of response patterns of key antioxidants that provide the molecular basis for the link between survival and temperature stress in Symbiodinium types with low and high thermal thresholds. Health includes photosynthetic functioning and cellular growth. ROS scavenging refers to the main removal pathway of superoxide via superoxide dismutase (SOD) and ascorbate peroxidase (APX). Components of the glutathione system that affect the glutathione redox state in addition to glutathione synthesis are indicated as Recycling (glutathione reductase; GR) and Damage (glutathione S-transferase; GST)

In addition to these differences in activity patterns of the antioxidant network, individual enzyme activities that determine the steady-state levels of particular ROS were considerably different between Symbiodinium types (Chapters 2 and 4). While the antioxidant activity of symbionts in hospite is affected by hostspecific features that affect the light environment such as tissue thickness, the presence of UV-shielding compounds or symbiont density (Shick et al., 1995, Dunlap \& Shick, 1998, Loya et al., 2001), Symbiodinium cultures allow an assessment under similar experimental conditions, free from host effects. This thesis agrees with previous studies regarding the differential activity of SOD between types (Lesser, 2011, McGinty et al., 2012), but shows that differences in total activity are much smaller for SOD than other antioxidants. For example, 
APX activity differed by a factor of 2-3 and total glutathione content differed by up to a factor of five (Chapter 2) or ten (Krämer et al., 2012). Even magnitude scale differences in glutathione S-transferase activity between Symbiodinium B1 and Symbiodinium E and F1 were observed (Chapter 2). Protective photosynthetic processes such as xanthophyll cycling or D1-repair, which affect the rate of ROS generation in the chloroplast, differ between Symbiodinium types (Warner \& Berry-Lowe, 2006, Takahashi et al., 2009, Ragni et al., 2010). The downstream pathways that scavenge ROS (primarily superoxide and hydrogen peroxide), though similar in mechanism, consequently express variability in their throughput. The differing baseline activities of these enzymes in Symbiodinium types are likely a reflection of this and contribute to the type-specific differences in the steady-state levels of ROS observed previously (McGinty et al., 2012).

Differences in baseline activities were much greater for network elements downstream of SOD (Chapter 2). Though SOD activity was measured as global activity (i.e. including all isoforms), it was possible to show that this finding is potentially related to the high degree of sequence conservation for SOD isoforms between distant Symbiodinium types (Chapter 3 ). Identities of $\geq 95 \%$ at the amino acid level suggest that most, if not all Symbiodinium types use the same molecular form of SOD. Complete conservation of functionally important residues and overall structure, indicates similar physicochemical properties of SODs in genetically distant types. Thus, enzymatic properties with regard to substrate affinity and turnover rate, as well as molecular stability at a higher temperature, are probably very similar. Further characterization of these isoforms is needed, but based on the findings in the Chapters 2 and 3, little selective advantage between types can be expected on the basis of superoxide dismutase activity, unless it relates to the ability to increase its expression under thermal stress.

In contrast, both peroxidases investigated here showed more amino acid substitutions and thus a higher degree of modification between types. Structural modification, with alteration of amino acid residues involved in substrate and cofactor binding, can alter catalytic rates and activity of these enzymes (reviewed in Gutteridge \& Thornton, 2005), and might therefore be under positive selective pressure. The study of Ladner et al. (2012) used ratios of synonymous (i.e. change in amino acid) versus non-synonymous (i.e. no change in amino acid) 
nucleotide substitutions per site as an indicator for genes that have been under positive selection. However, my approach was to analyse sequences with respect to substitutions in residues involved in binding of substrate or co-factors, to highlight the impact of Symbiodinium diversity on structural features. Further research is clearly needed to bridge the gap between sequence variations and their functional consequences. Purifying and characterizing these enzymes will allow further investigations of the effects of temperature on the stability and substrate affinity ( $K_{m}$ values) of these proteins. The amount of data generated here also opens the possibility for work on post-transcriptional gene silencing to more thoroughly investigate the role and regulation of individual parts of the system, as this was not possible with the gene expression experiment conducted here.

\section{Is genetic redundancy a driver of variability in antioxidant defences downstream of superoxide dismutase?}

This thesis has shown that for Symbiodinium in culture, differences in survival under thermal stress cannot be related directly to the activity of individual antioxidants, but rather to mechanistic changes in the antioxidant network, and primarily to more downstream processes. Differences in activity and structure of primary enzymatic antioxidants such as SODs were comparably small between Symbiodinium types. This might be due to either lower mutation rates or a higher selective pressure on their conserved molecular function. The reason why the link between genetic divergence, variation in the antioxidant system, and thermal sensitivity in Symbiodinium types can be found in more downstream pathways, probably relates to a degree of genetic redundancy with respect to the removal of secondary ROS, such as hydrogen peroxide. Genetic redundancy means that two or more genes have the same proteomic function and modification or inactivation of one of these genes has no or only a small effect on the biological phenotype (Nowak et al., 1997). In the context of antioxidants, this redundancy contains numerical (most cellular compartments have their own set of antioxidants) and functional aspects (multiple enzymes scavenge hydrogen peroxide, relying on different cofactors). Comparative studies of Arabidopsis knockout mutants that have suppressed expression of individual antioxidants, 
have shown that the concerted antioxidant response is highly specific to the type of stress (flexibility), and that other components of the antioxidant network tend to be overexpressed in order to compensate for specific deficiencies (i.e. redundancy) (Mittler et al., 2004). In tobacco plants, a lack or reduced expression of CAT can be compensated for by increased expression of APX or glutathione peroxidase (GPX) (Willekens et al., 1997), whereas APX deficiency results in increased SOD, CAT and GR activity (Rizhsky et al., 2002). For Symbiodinium, this functional redundancy has not been shown yet, partially due to the lack of available sequences for most of these genes. However, on an evolutionary scale, this redundancy might have provided the opportunity for the development of variations in downstream antioxidant responses and sequence alteration in peroxidase between Symbiodinium types.

This thesis provides evidence for functional variation in the glutathione system between thermally sensitive and tolerant Symbiodinium types, and provides evidence for sequence variation in two peroxidases that could become evolutionarily relevant if they alter catalytic properties of hydrogen peroxide removal. Both of these enzymatic variations could contribute to an evolutionary advantage for some Symbiodinium types, as they provide the means to more effectively detoxify ROS under high temperatures or high light.

\section{Is there a link between heating rate and the temporal onset of ROS production in both partners?}

The findings obtained from the culture experiment (Chapter 2) agree with the theory that photo-oxidative stress initiates cell death in thermally stressed Symbiodinium (reviewed in Chapter 1). However, considerable variability between replicates was observed for individual antioxidants, perhaps due to different heating rates between the replicate experimental trials; these ranged from $1.1-1.8^{\circ} \mathrm{C}$ per hour for the $33^{\circ} \mathrm{C}$ treatment (Chapter 2). Applying a lower, but more stable heating rate of $1^{\circ} \mathrm{C}$ per hour, delayed the overall antioxidant response in the same Symbiodinium type (B1) in culture (Chapter 3), and significant effects after three days were only measurable for APX activity. Coral reef-flat communities can experience temperature changes of $1^{\circ} \mathrm{C}$ per hour during spring tides (Berkelmans \& Willis, 1999), however temperature changes 
relevant to mass bleaching are usually lower, and therefore heating rates of $1^{\circ} \mathrm{C}$ per day were applied for the in hospite study (Chapter 4). Surprisingly, antioxidant responses of the holobiont under these conditions contradicted the findings of a number of previous studies in that no overall upregulation was observed in the symbiont, and that antioxidant processes in the host preceded photosynthetic dysfunction (outlined in Chapter 4).

While it appears reasonable to assume that heating rates affect the temporal response of photosynthetic dysfunction, this aspect has been largely overlooked in the literature. However, Middlebrook et al. (2010) demonstrated that lowering heating rates from 1 to $0.5^{\circ} \mathrm{C}$ per day considerably delays the photosynthetic response of the coral Acropora formosa with faster heating causing more immediate dysfunction. If lower ramping rates delay photosynthetic decline (and therefore the initiation of photo-oxidative stress in the symbiont) relative to the host's thermal response, then host-generated hydrogen peroxide may interfere with and/or inhibit the symbiont's antioxidative network (e.g., feedback inhibition of APX and some SOD isoforms). This assumes that the cellular hydrogen peroxide level in the host's cytosol initially exceeds the steady-state concentration in the symbiont under thermal stress, when low heating rates are applied. This study has shown a higher turnover of hydrogen peroxide in the host than in the symbiont, but no studies have investigated the effect of temperature on the actual concentration of cellular hydrogen peroxide in each partner. Further studies will also have to examine the role that heating rates play in the temporal response of antioxidant defences and ROS concentrations in both partners. A hydrogen peroxide-mediated impact of the host on the symbiont's physiology under thermal stress (at low heating rates) would be consistent with the patterns described in Chapter 4 and Hawkins et al. (in press), where host antioxidant and caspase-like activities, respectively, were elevated prior to photosynthetic dysfunction.

Increased host ROS levels and their potential impact on the symbiont population should also be considered in the context of pathogen recognition and immunity responses in the coral. Symbiodinium cells are essentially located within arrested phagosomes (Fitt \& Trench, 1983), and increased production of nitric oxide in the host under thermal stress has been associated with an apoptotic-like cell 
pathway that also plays a role in the initial winnowing process and removal of unsuitable symbiont types (Perez \& Weis, 2006, Dunn \& Weis, 2009, Hawkins et al., 2013). Thus, the breakdown of host-symbiont recognition mechanisms might lead to the maturation of phagosomes to phagolysosomes (Chen et al., 2005b). Antipathogenic responses in mature phagolysosomes usually involve the generation of ROS and nitric oxide, partially through membrane-bound NADPH oxidase (Nox), which has been found in the genome of the cnidarian Nematostella vectensis (Sumimoto, 2008, Wink et al., 2011). The role of ROS in pathogen recognition has not been investigated in depth in corals, but increased peroxidase activity (measured as the holobiont response) to a fungal infection has been observed in the symbiotic sea fan Gorgonia ventalina (Mydlarz \& Harvell, 2007). It therefore seems plausible that hydrogen peroxide might form part of the host's antipathogenic response towards its symbionts, particularly when it has sufficient CAT activity to attenuate any self-harm.

\section{Can the symbiont challenge the host with ROS?}

Enzymatic scavenging capacities of hydrogen peroxide by CAT have been shown to be consistently higher in the host than in the symbiont, whereas SOD activities are more similar though slightly higher in the symbiont (Chapter 4, Lesser et al., 1990, Shick et al., 1995, Yakovleva et al., 2004, Levy et al., 2006, Higuchi et al., 2008, Higuchi et al., 2012) (Figure 5.2A). Assuming that both compartments have low cellular steady-state concentrations of superoxide and hydrogen peroxide, leakage of either compound to the other partner potentially represents an oxidative challenge. While superoxide is short-lived and cannot permeate through intact membranes (Takahashi \& Asada, 1983, Winterbourn, 2008), necrotic Symbiodinium cells that may release superoxide pose a potential threat to the host since high levels of superoxide can also react with nitric oxide $\left(\mathrm{NO}^{\bullet}\right)$, generating peroxynitrite (ONOO*-) (Radi et al., 2001, Halliwell \& Gutteridge, 2007). This highly reactive compound can damage enzymes and is potentially involved in the molecular bleaching process (Perez \& Weis, 2006, Hawkins et al., 2013, Hawkins \& Davy, 2013).

As outlined in Chapter 1, hydrogen peroxide is capable of passing through membranes and the leakage of this compound from thermally-compromised 
symbionts to the host tissue is central to the Oxidative Theory of Coral Bleaching (Downs et al., 2002, Venn et al., 2008, Weis, 2008). If the symbiont initiates the bleaching cascade and determines the bleaching susceptibility of the coral holobiont, then two assumptions must be met. First, the production of ROS will not exceed the scavenging rate in thermally tolerant symbionts. Second, the amount of ROS released by thermally compromised symbionts is sufficient to challenge the host's antioxidative system. However, this thesis provides no evidence for increased symbiont antioxidant defences prior to thermal bleaching in tolerant corals relative to susceptible corals. Indeed, such differences were only found in the host response, in the form of increased CAT activity and the initiation of apoptotic events in susceptible corals (Chapter 4, Hawkins et al., 2014).

Measurements of steady-state fluxes of hydrogen peroxide in the host and symbiont are needed to elucidate the potential of the symbiont to harm the host via leakage of this ROS. As a first step, however, the upper vales for the release of ROS (as hydrogen peroxide) by injured Symbiodinium (Mydlarz \& Jacobs, 2004) will be used here to model potential events. If all cells were to be physically injured at the same time, the estimated rate of released hydrogen peroxide at symbiont densities comparable to those in $A$. millepora and $M$. digitata would be 0.1-0.2 $\mu \mathrm{mol} \mathrm{min}{ }^{-1} \mathrm{~cm}^{-2}\left(\sim 0.1 \mathrm{pmol} \mathrm{H}_{2} \mathrm{O}_{2} \min ^{-1}\right.$ cell $^{-1} \mathrm{x} \sim 1-210^{6}$ cells $\left.\mathrm{cm}^{-2}\right)$. In contrast, total CAT activity of the host for the same surface area would be at least $60 \mu \mathrm{mol} \mathrm{min}{ }^{-1}\left(0.5-1.0 \mathrm{mg}\right.$ protein $\mathrm{cm}^{-2} \times 120 \mu \mathrm{mol} \mathrm{min}^{-1} \mathrm{mg}$ protein $\left.{ }^{-1}\right)$. Thus, the maximum release of hydrogen peroxide from the symbiont population and the minimal scavenging capacity by host CAT differ by three orders of magnitude (or $\sim 0.2 \%$ ). Even higher values of hydrogen peroxide production, as measured by McGinty et al. (2012) (maximum 2 pmol $\mathrm{H}_{2} \mathrm{O}_{2} \min ^{-1}$ cell ${ }^{-1}$ for Symbiodinium $\mathrm{C} 1$ ), would still amount to only $3.3 \%$ of the host's scavenging capacity. Values provided by these latter authors are, however, debatable, since the probe concentration used (0.67 $\mathrm{mM}$ dichlorofluorescin diacetate, DCFDA) was likely cytotoxic and above the recommended concentration (10 $\mu \mathrm{M}$, Halliwell \& Gutteridge, 2007). It is also of note that the imbalance in the scavenging capacity of the symbiont and host in Acropora millepora and $P$. damicornis increased further under thermal stress (Figure 5.2B). 

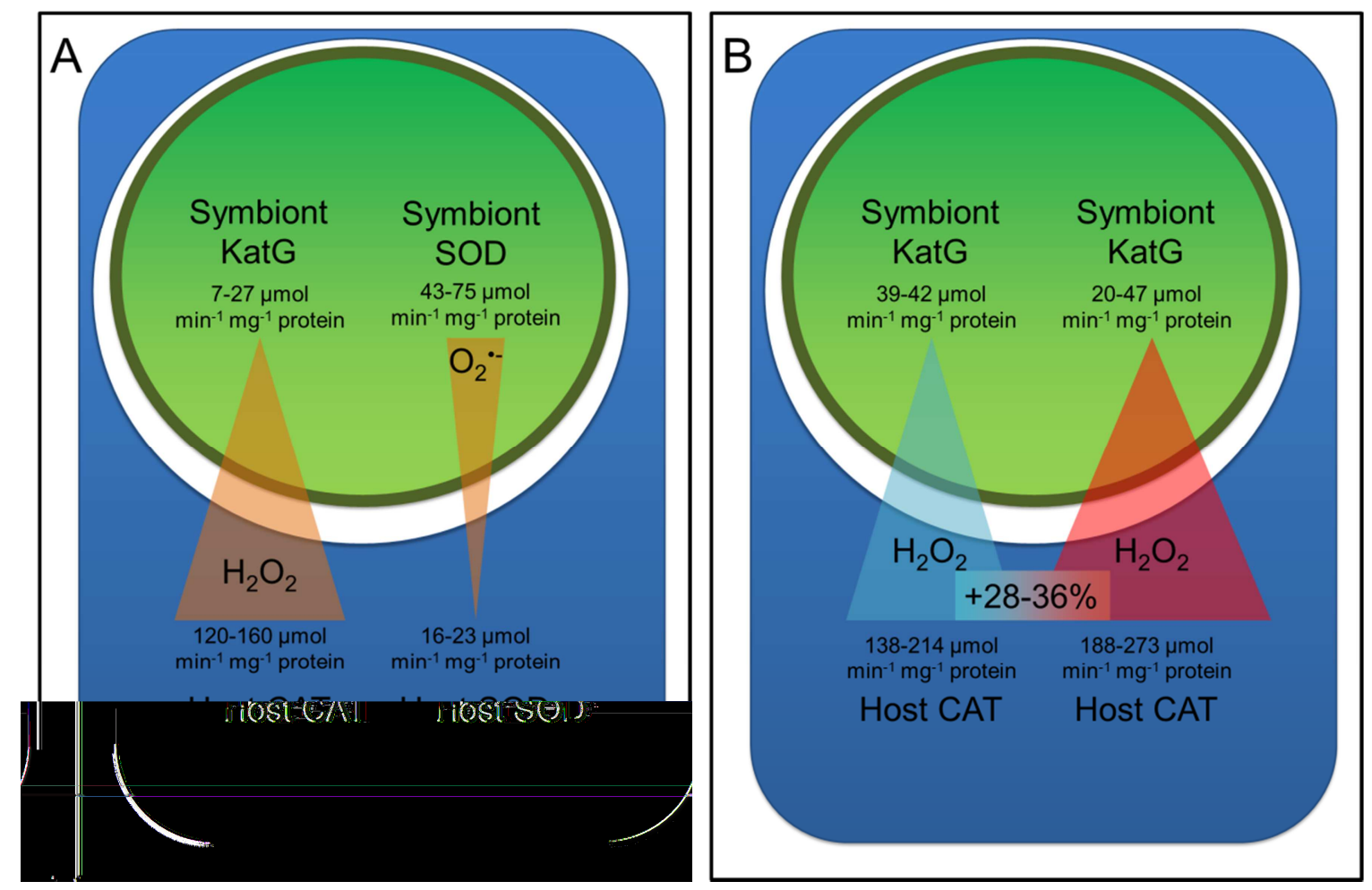

Figure 5.2. Schematic depiction of gradients for scavenging of hydrogen peroxide and superoxide in host and symbiont.

(A) Gradients for superoxide and hydrogen peroxide scavenging in host (blue) and symbiont (green) compartment at $28^{\circ} \mathrm{C}$. Values are based on the range of specific activities for superoxide dismutase (SOD), catalase (CAT), and catalase peroxidase (KatG) from the corals Acropora millepora, Montipora digitata and Pocillopora damicornis. (B) Changes in hydrogen peroxide scavenging in $A$. millepora and $P$. damicornis between $28^{\circ} \mathrm{C}$ (bright blue triangle) and $33^{\circ} \mathrm{C}$ (red triangle).

\section{Cell biology is the key to understanding the thermal response of corals}

Our understanding of the cellular and molecular biology of corals has made substantial advances in the past 25 years, especially in the areas of Symbiodinium diversity, coral stress physiology, inter-partner recognition, metabolic relationships and symbiotic breakdown (Brown, 1997, Coffroth \& Santos, 2005, Weis et al., 2008, Yellowlees et al., 2008, Lesser, 2011, Davy et al., 2012). Nevertheless, as our awareness of the complexity and interdependence of the host-symbiont relationship increases so too does the realisation that we still have a huge amount to learn about its function.

While the research presented in this thesis provides further insight into the mechanisms that underlie thermal tolerance in corals, it also raises numerous 
questions about the potential roles of other antioxidant systems, their regulation at the transcriptomic level, and their ultimate importance in explaining differential bleaching susceptibilities. Indeed, while physiological differences between Symbiodinium types (e.g., Iglesias-Prieto et al., 1992, Warner et al., 1996, Bhagooli \& Hidaka, 2003, Fabricius et al., 2004, Suggett et al., 2008, Takahashi et al., 2009, Ragni et al., 2010) and the role of the host in determining bleaching susceptibility (Gates et al., 1992, Dove, 2004, Abrego et al., 2008, Fitt et al., 2009, Barshis et al., 2010, Tchernov et al., 2011) are becoming clearer, there is still a great need for experiments that elucidate the cellular and molecular processes involved. There is also a need for experiments that test the effects of thermal acclimation and heating rates on these cellular and molecular processes, as these play a fundamental role in the thermal stress response of corals (Middlebrook et al., 2008, Middlebrook et al., 2010).

As predicted by climate change scenarios, corals will have to adapt by increasing their thermal tolerance by $0.2-1.0^{\circ} \mathrm{C}$ per decade (Donner et al., 2005). Individual reef systems have been shown to be extremely resilient under the absence of local human stressors (Gilmour et al., 2013), however the combined effect of local and global environmental pressures will likely prove detrimental for most reefs in the next 50 to 100 years (Hoegh-Guldberg et al., 2007, Carpenter et al., 2008, Frieler et al., 2012, Hoegh-Guldberg, 2012). In conclusion, cell biology is the key to understanding the thermal response of corals, but it is the human attitude towards environmental stewardship that will decide whether the present complexity of coral reef ecosystems will survive into the future. 
- 116 - 


\section{References}

Abascal, F., Zardoya, R. \& Posada, D. 2005. ProtTest: selection of best-fit models of protein evolution. Bioinformatics 21:2104-05.

Abrego, D., Ulstrup, K. E., Willis, B. L. \& van Oppen, M. J. H. 2008. Speciesspecific interactions between algal endosymbionts and coral hosts define their bleaching response to heat and light stress. Proc $R$ Soc $B$ 275:227382.

AIMS 2013. Table generated 25th March 2013 using Reef Monitoring, Heron Island HERFL1 Water Temperature @1m Reef Flat Site 1. Long term Monitoring and Data Centre, AIMS. Viewed 25th March 2013 $<$ http://data.aims.gov.au/aimsrtds/datatool.xhtml>.

Allemand, D., Tambutté, É., Zoccola, D. \& Tambutté, S. 2011. Coral calcification, cells to reefs. In: Dubinksy, Z. \& Stambler, N. [Eds.] Coral reefs: An ecosystem in transition. Springer, Dordrecht Heidelberg London New York, pp. 119-50.

Alscher, R. G., Erturk, N. \& Heath, L. S. 2002. Role of superoxide dismutases (SODs) in controlling oxidative stress in plants. J Exp Bot 53:1331-41.

Altschul, S. F., Gish, W., Miller, W., Myers, E. W. \& Lipman, D. J. 1990. Basic local alignment search tool. $J$ Mol Biol 215:403-10.

Andersen, C. L., Jensen, J. L. \& Ørntoft, T. F. 2004. Normalization of real-time quantitative reverse transcription-PCR data: a model-based variance estimation approach to identify genes suited for normalization, applied to bladder and colon cancer data sets. Cancer Res 64:5245-50.

Apel, K. \& Hirt, H. 2004. Reactive oxygen species: metabolism, oxidative stress, and signal transduction. Annu. Rev. Plant Physiol. Plant Mol. Biol. 55:37399.

Arasimowicz-Jelonek, M. \& Floryszak-Wieczorek, J. 2011. Understanding the fate of peroxynitrite in plant cells - From physiology to pathophysiology. Phytochemistry:681-88.

Asada, K. 1984. Chloroplasts: Formation of active oxygen and its scavenging. Methods Enzymol 105:422-29.

Asada, K. 2000. The water-water cycle as alternative photon and electron sinks. Philos Trans $R$ Soc B Biol Sci 355:1419-31.

Asada, K. 2006. Production and scavenging of reactive oxygen species in chloroplasts and their functions. Plant Physiol 141:391-96.

Baird, A. H., Bhagooli, R., Ralph, P. J. \& Takahashi, S. 2009. Coral bleaching: the role of the host. Trends Ecol Evol 24:16-20.

Baker, A. C. 2003. Flexibility and specificity in coral-algal symbiosis: Diversity, ecology, and biogeography of Symbiodinium. Annu Rev Ecol Evol Syst 34:661-89.

Baker, A. C. 2004. Corals' adaptive response to climate change. Nature 430:741. 
Baker, A. C., Smith, L. W. \& Wirshing, H. H. 2008. Coral symbiont diversity along a seawater temperature gradient. Tester's Symposium. Honolulu, Hawaii, 22-Mar-08.

Baker, M. A., G.J., C. \& Zaman, A. 1990. Microtiter plate assay for the measurements of glutathione and glutathione disulfide in large numbers of biological samples. Anal Biochem 190:360-65.

Baker, R. D., Cook, C. O. \& Goodwin, D. C. 2004. Properties of catalaseperoxidase lacking its C-terminal domain. Biochem Biophys Res Commun 320:833-39.

Barshis, D. J., Stillman, J. H., Gates, R. D., Toonen, R. J., Smith, L. W. \& Birkeland, C. 2010. Protein expression and genetic structure of the coral Porites lobata in an environmentally extreme Samoan back reef: does host genotype limit phenotypic plasticity? Mol Ecol 19:1705-20.

Bayer, T., Aranda, M., Sunagawa, S., Yum, L. K., DeSalvo, M. K., Lindquist, E., Coffroth, M. A., Voolstra, C. R. \& Medina, M. 2012. Symbiodinium transcriptomes: Genome insights into the dinoflagellate symbionts of reefbuilding corals. PLOS ONE 7:e35269.

Beauchamp, C. \& Fridovich, I. 1971. Superoxide dismutase: Improved assays and an assay applicable to acrylamide gels. Anal Biochem 44:276-87.

Beers, R. \& Sizer, I. W. 1952. A spectrophotometric method for measuring the breakdown of hydrogen peroxide by catalase. J Biol Chem 195:133-40.

Benson, D. A., Karsch-Mizrachi, I., Lipman, D. J., Ostell, J. \& Sayers, E. W. 2010. GenBank. Nucleic Acids Res 38:D46-D51.

Berkelmans, R. \& van Oppen, M. J. H. 2006. The role of zooxanthellae in the thermal tolerance of corals: a 'nugget of hope' for coral reefs in an era of climate change. Proc $R$ Soc B 273:2305-12.

Berkelmans, R. \& Willis, B. 1999. Seasonal and local spatial patterns in the upper thermal limits of corals on the inshore Central Great Barrier Reef. Coral Reefs 18:219-28.

Berumen, M. L. \& Pratchett, M. S. 2006. Recovery without resilience: persistent disturbance and long-term shifts in the structure of fish and coral communities at Tiahura Reef, Moorea. Coral Reefs 25:647-53.

Bhagooli, R. \& Hidaka, M. 2003. Comparison of stress susceptibility of in hospite and isolated zooxanthellae among five coral species. J Exp Mar Biol Ecol 291:181-97.

Bienert, G. P., Schjoerring, J. K. \& Jahn, T. P. 2006. Membrane transport of hydrogen peroxide. Biochim Biophys Acta 1758:994-1003.

Black, N. A., Voellmy, R. \& Szmant, A. M. 1995. Heat shock protein induction in Montastraea faveolata and Aiptasia pallida exposed to elevated temperatures. Biol Bull 188:234-40.

Boldt, L., Yellowlees, D. \& Leggat, W. 2008. Measuring Symbiodinium sp. gene expression patterns with quantitative real-time PCR. Proceedings of the 11th International Coral Reef Symposium. Fort Lauderdale, FL, USA, 7-11 July 2008, pp. 118-22. 
Bou-Abdallah, F., Chasteen, N. D. \& Lesser, M. P. 2006. Quenching of superoxide radicals by green fluorescent protein. Biochim Biophys Acta 1760:1690-95.

Bouchard, J. N. \& Yamasaki, H. 2008. Heat stress stimulates nitric oxide production in Symbiodinium microadriaticum: A possible linkage between nitric oxide and the coral bleaching phenomenon. Plant Cell Physiol 49:641-52.

Brown, B. E. 1997. Coral bleaching: causes and consequences. Coral Reefs 16:129-38.

Brown, B. E., Ambarsari, I., Warner, M. E., Fitt, W. K., Dunne, R. P., Gibb, S. W. \& Cummings, D. G. 1999. Diurnal changes in photochemical efficiency and xanthophyll concentrations in shallow water reef corals: evidence for photoinhibition and photoprotection. Coral Reefs 19:99-105.

Bruno, J. F. \& Selig, E. R. 2007. Regional decline of coral cover in the IndoPacific: Timing, extent, and subregional comparisons. PLOS ONE 2:e711.

Buchanan, B. B., Gruissem, W. \& Jones, R. L. 2000. Biochemistry and molecular biology of plants. American Society of Plant Physiologists, Rockville, MD, USA.

Buddemeier, R. W., Baker, A. C., Fautin, D. G. \& Jacobs, J. R. 2004. The Adaptive Hypothesis of Bleaching. In: Rosenberg, E. \& Loya, Y. [Eds.] Coral Health and Disease. Springer-Verlag, Berlin, Germany.

Buddemeier, R. W. \& Fautin, D. G. 1993. Coral bleaching as an adaptive mechanism. Bioscience 43:320-26.

Burke, L. M., Reytar, K., Spalding, M. \& Perry, A. 2011. Reefs at risk revisited. World Resources Institute Washington, DC,

Cadenas, E. 1995. Mechanisms of oxygen activation and reactive oxygen species detoxification. In: Ahmad, S. [Ed.] Oxidative Stress and Antioxidant Defenses in Biology. Chapman \& Hall, New York, USA.

Cantin, N. E., van Oppen, M. J. H., Willis, B. L., Mieog, J. C. \& Negri, A. P. 2009. Juvenile corals can acquire more carbon from high-performance algal symbionts. Coral Reefs 28:405-14.

Carpenter, K. E., Abrar, M., Aeby, G., Aronson, R. B., Banks, S., Bruckner, A., Chiriboga, A., Cortés, J., Delbeek, J. C. \& DeVantier, L. 2008. One-third of reef-building corals face elevated extinction risk from climate change and local impacts. Science 321:560-63.

Cesar, H. S. J. \& Van Beukering, P. 2004. Economic valuation of the coral reefs of Hawai'i. Pac Sci 58:231-42.

Chen, C. A., Wang, J.-T., Fang, L.-S. \& Yang, Y.-W. 2005a. Fluctuating algal symbiont communities in Acropora palifera (Scleractinia: Acroporidae) from Taiwan. Mar Ecol Prog Ser 295:113-21. 
Chen, M.-C., Hong, M.-C., Huang, Y.-S., Liu, M.-C., Cheng, Y.-M. \& Fang, L.-S. 2005b. ApRab11, a cnidarian homologue of the recycling regulatory protein Rab11, is involved in the establishment and maintenance of the Aiptasia-Symbiodinium endosymbiosis. Biochem Biophys Res Commun 338:1607-16.

Coffroth, M. A. \& Santos, S. R. 2005. Genetic Diversity of Symbiotic Dinoflagellates in the Genus Symbiodinium. Protist 156:19-34.

Colasanti, M., Persichini, T. \& Venturini, G. 2010. Nitric oxide pathway in lower metazoans. Nitric Oxide 23:94-100.

Conservation International 2008. Economic values of coral reefs, mangroves, and seagrasses: A global compilation. Center for Applied Biodiversity Science, Conservation International, Arlington, VA, USA,

Consortium, U. 2013. Update on activities at the Universal Protein Resource (UniProt) in 2013. Nucleic Acids Res 41:D43-D47.

Cooper, G. M. \& Brown, C. D. 2008. Qualifying the relationship between sequence conservation and molecular function. Genome Res 18:201-05.

Cribb, A. E., Leeder, J. S. \& Spielberg, S. P. 1989. Use of a microplate reader in an assay of glutathione reductase using 5, 5'-dithiobis (2-nitrobenzoic acid). Anal Biochem 183:195-96.

Császár, N., Seneca, F. \& Van Oppen, M. 2009. Variation in antioxidant gene expression in the scleractinian coral Acropora millepora under laboratory thermal stress. Mar Ecol Prog Ser 392:102.

D'Autréaux, B. \& Toledano, M. B. 2007. ROS as signalling molecules: mechanisms that generate specificity in ROS homeostasis. Nat Rev Mol Cell Biol 8:813-24.

Davletova, S., Rizhsky, L., Liang, H., Shengqiang, Z., Oliver, D. J., Coutu, J., Shulaev, V., Schlauch, K. \& Mittler, R. 2005. Cytosolic ascorbate peroxidase 1 is a central component of the reactive oxygen gene network of Arabidopsis. The Plant Cell 17:268-81.

Davy, S. K., Allemand, D. \& Weis, V. M. 2012. Cell biology of cnidariandinoflagellate symbiosis. Microbiol Mol Biol Rev 76:229-61.

Dayer, R., Fischer, B. B., Eggen, R. I. L. \& Lemaire, S. D. 2008. The peroxiredoxin and glutathione peroxidase families in Chlamydomonas reinhardtii. Genetics 179:41-57.

deSalvo, M. K., Voolstra, C. R., Sunagawa, S., Schwarz, J. A., Stillman, J. H., Coffroth, M. A., Szmant, A. M. \& Medina, M. 2008. Differential gene expression during thermal stress and bleaching in the Caribbean coral Montastraea faveolata. Mol Ecol 17:3952-71.

Dietz, K.-J., Horling, F., König, J. \& Baier, M. 2002. The function of the chloroplast 2-cysteine peroxiredoxin in peroxide detoxification and its regulation. J Exp Bot 53:1321-29.

Donner, S. D., Skirving, W. J., Little, C. M., Oppenheimer, M. \& Hoegh Guldberg, O. 2005. Global assessment of coral bleaching and required rates of adaptation under climate change. Glob Change Biol 11:2251-65. 
Dove, S. 2004. Scleractinian corals with photoprotective host pigments are hypersensitive to thermal bleaching. Mar Ecol Prog Ser 272:99-116.

Downs, C. A., Fauth, J. E., Halas, J. C., Dustan, P., Bemiss, J. \& Woodley, C. M. 2002. Oxidative stress and seasonal coral bleaching. Free Radical Biol Med 33:533-43.

Downs, C. A., Fauth, J. E., Robinson, C. E., Curry, R., Lanzendorf, B., Halas, J. C., Halas, J. \& Woodley, C. M. 2005. Cellular diagnostics and coral health: Declining coral health in the Florida Keys. Mar Pollut Bull 51:558-69.

Downs, C. A., Mueller, E., Phillips, S., Fauth, J. E. \& Woodley, C. M. 2000. A molecular biomarker system for assessing the health of coral (Montastraea faveolata) during heat stress. Mar Biotechnol 2:533-44.

Dufernez, F., Derelle, E., Noël, C., Sanciu, G., Mantini, C., Dive, D., SoyerGobillard, M.-O., Capron, M., Pierce, R. J. \& Wintjens, R. 2008. Molecular characterization of iron-containing auperoxide dismutases in the heterotrophic dinoflagellate Crypthecodinium cohnii. Protist 159:223-38.

Dunlap, W. C. \& Shick, J. M. 1998. Ultraviolet radiation-absorbing mycosporinelike amino acids in coral reef organisms: A biochemical and environmental perspective. J Phycol 34:418-30.

Dunlap, W. C. \& Yamamoto, Y. 1995. Small-molecule antioxidants in marine organisms: antioxidant activity of mycosporine-glycine. Comp Biochem Physiol B Biochem Mol Biol 112:105-14.

Dunn, S. R., Bythell, J. C., le Tissier, M. D. A., Burnett, W. J. \& Thomason, J. C. 2002. Programmed cell death and cell necrosis activity during hyperthermic stress-induced bleaching of the symbiotic sea anemone Aiptasia sp. J Exp Mar Biol Ecol 272:29-53.

Dunn, S. R., Pernice, M., Green, K., Hoegh-Guldberg, O. \& Dove, S. G. 2012. Thermal stress promotes host mitochondrial degradation in symbiotic cnidarians: are the batteries of the reef going to run out? PLOS ONE 7:e39024.

Dunn, S. R., Phillips, W. S., Green, D. R. \& Weis, V. M. 2007a. Knockdown of actin and caspase gene expression by RNA interference in the symbiotic anemone Aiptasia pallida. Biol Bull 212:250-58.

Dunn, S. R., Phillips, W. S., Spatafora, J. W., Green, D. R. \& Weis, V. M. 2006. Highly conserved caspase and Bcl-2 homologues from the sea anemone Aiptasia pallida: lower metazoans as models for the study of apoptosis evolution. J Mol Evol 63:95-107.

Dunn, S. R., Schnitzler, C. E. \& Weis, V. M. 2007b. Apoptosis and autophagy as mechanisms of dinoflagellate symbiont release during cnidarian bleaching: every which way you lose. Proc $R$ Soc $B$ 274:3079-85.

Dunn, S. R., Thomason, J. C., le Tissier, M. D. A. \& Bythell, J. C. 2004. Heat stress induces different forms of cell death in sea anemones and their endosymbiotic algae depending on temperature and duration. Cell Death Differ 11:1213-22. 
Dunn, S. R. \& Weis, V. M. 2009. Apoptosis as a post-phagocytic winnowing mechanism in a coral-dinoflagellate mutualism. Environ Microbiol 11:26876.

Dykens, J. A. \& Shick, J. M. 1982. Oxygen production by endosymbiotic algae controls superoxide dismutase activity in their animal host. Nature 297:579-80.

Dykens, J. A. \& Shick, J. M. 1984. Photobiology of the symbiotic sea anemone, Anthopleura elegantissima: defenses against photodynamic effects, and seasonal photoacclimatization. Biol Bull 167:683-97.

Dykens, J. A., Shick, J. M., Benoit, C., Buettner, G. R. \& Winston, G. W. 1992. Oxygen radical production in the sea anemone Anthopleura elegantissima and its endosymbiotic algae. J Exp Biol 168:219-41.

Edge, S. E., Morgan, M. B. \& Snell, T. W. 2008. Temporal analysis of gene expression in a field population of the Scleractinian coral Montastraea faveolata. J Exp Mar Biol Ecol 355:114-24.

Edmunds, P. J. \& Gates, R. D. 2003. Has coral bleaching delayed our understanding of fundamental aspects of coral-dinoflagellate symbioses? Bioscience 53:976-80.

Elmore, S. 2007. Apoptosis: a review of programmed cell death. Toxicol Pathol 35:495-516.

Elstner, E. F. 1982. Oxygen activation and oxygen toxicity. Annu. Rev. Plant Physiol. Plant Mol. Biol. 33:73-96.

Emanuelsson, O., Nielsen, H., Brunak, S. \& von Heijne, G. 2000. Predicting subcellular localization of proteins based on their $\mathrm{N}$-terminal amino acid sequence. J Mol Biol 300:1005-16.

Enríquez, S., Méndez, E. R. \& Iglesias-Prieto, R. 2005. Multiple scattering on coral skeletons enhances light absorption by symbiotic algae. Limnol Oceanogr 50:1025-32.

Fabricius, K. E., Mieog, J. C., Colin, P. L., Idip, D. \& van Oppen, M. J. H. 2004. Identity and diversity of coral endosymbionts (zooxanthellae) from three Palauan reefs with contrasting bleaching, temperature and shading histories. Mol Ecol 13:2445-58.

Fagan, T., Morse, D. \& Hastings, J. W. 1999. Circadian synthesis of a nuclearencoded chloroplast glyceraldehyde-3-phosphate dehydrogenase in the dinoflagellate Gonyaulax polyedra is translationally controlled. Biochemistry 38:7689-95.

Fang, L., Huang, S. \& Lin, K. 1997. High temperature induces the synthesis of heat-shock proteins and the elevation of intracellular calcium in the coral Acropora grandis. Coral Reefs 16:127-31.

Fawal, N., Li, Q., Savelli, B., Brette, M., Passaia, G., Fabre, M., Mathé, C. \& Dunand, C. 2013. PeroxiBase: a database for large-scale evolutionary analysis of peroxidases. Nucleic Acids Res 41:D441-D44.

Felsenstein, J. 1985. Confidence limits on phylogenies: An approach using the bootstrap. Evolution 39:783-91. 
Ferri, K. F. \& Kroemer, G. 2001. Organelle-specific initiation of cell death pathways. Nat Cell Biol 3:E255-E63.

Ferrier-Pagès, C., Richard, C., Forcioli, D., Allemand, D., Pichon, M. \& Shick, J. M. 2007. Effects of temperature and UV radiation increases on the photosynthetic efficiency in four scleractinian coral species. Biol Bull 213:76-87.

Finkel, T. \& Holbrook, N. J. 2000. Oxidants, oxidative stress and the biology of ageing. Nature 408:239-47.

Fisher, P. L., Malme, M. K. \& Dove, S. 2012. The effect of temperature stress on coral-Symbiodinium associations containing distinct symbiont types. Coral Reefs 31:473-85.

Fitt, W. K., Brown, B. E., Warner, M. E. \& Dunne, R. P. 2001. Coral bleaching: interpretation of thermal tolerance limits and thermal thresholds in tropical corals. Coral Reefs 20:51-65.

Fitt, W. K., Gates, R. D., Hoegh-Guldberg, O., Bythell, J. C., Jatkar, A., Grottoli, A. G., Gomez, M., Fisher, P., LaJeunesse, T. C., Pantos, O., IglesiasPrieto, R., Franklin, D. J., Rodrigues, L. J., Torregiani, J. M., van Woesik, R. \& Lesser, M. P. 2009. Response of two species of Indo-Pacific corals, Porites cylindrica and Stylophora pistillata, to short-term thermal stress: The host does matter in determining the tolerance of corals to bleaching. $J$ Exp Mar Biol Ecol 373:102-10.

Fitt, W. K. \& Trench, R. K. 1983. Endocytosis of the symbiotic dinoflagellate Symbiodinium microadriaticum Freudenthal by endodermal cells of the scyphistomae of Cassiopeia xamachana and resistance of the algae to host digestion. J Cell Sci 64:195-212.

Flores-Ramírez, L. A. \& Liñán-Cabello, M. A. 2007. Relationships among thermal stress, bleaching and oxidative damage in the hermatypic coral, Pocillopora capitata. Comp Biochem Physiol C Toxicol Pharmacol 146:194-202.

Forêt, S., Kassahn, K. S., Grasso, L., Hayward, D., Iguchi, A., Ball, E. \& Miller, D. J. 2007. Genomic and microarray approaches to coral reef conservation biology. Coral Reefs 26:475-86.

Foyer, C. H., Lelandais, M. \& Kunert, K. J. 1994. Photooxidative stress in plants. Physiol Plant 92:696-717.

Foyer, C. H. \& Noctor, G. 2011. Ascorbate and glutathione: the heart of the redox hub. Plant Physiol 155:2-18.

Foyer, C. H. \& Shigeoka, S. 2011. Understanding oxidative stress and antioxidant functions to enhance photosynthesis. Plant Physiol 155:93-100.

Franco, R., Schoneveld, O. J., Pappa, A. \& Panayiotidis, M. I. 2007. The central role of glutathione in the pathophysiology of human diseases. Arch Physiol Biochem 113:234-58. 
Franklin, E. C., Stat, M., Pochon, X., Putnam, H. M. \& Gates, R. D. 2012. GeoSymbio: a hybrid, cloud-based web application of global geospatial bioinformatics and ecoinformatics for Symbiodinium-host symbioses. Mol Ecol Resour 12:369-73.

Frei, B., Kim, M. C. \& Ames, B. N. 1990. Ubiquinol-10 is an effective lipid-soluble antioxidant at physiological concentrations. Proc Natl Acad Sci U S A 87:4879-83.

Freudenthal, H. D. 1962. Symbiodinium gen. nov. and Symbiodinium microadriaticum sp. nov., a Zooxanthella : Taxonomy, life cycle, and morphology. J Protozool 9:45-52.

Fridovich, I. 1998. Oxygen toxicity: A radical explanation. J Exp Biol 201:1203-09.

Frieler, K., Meinshausen, M., Golly, A., Mengel, M., Lebek, K., Donner, S. \& Hoegh-Guldberg, O. 2012. Limiting global warming to $2^{\circ} \mathrm{C}$ is unlikely to save most coral reefs. Nat Clim Change.

Fryer, M. J., Andrews, J. R., Oxborough, K., Blowers, D. A. \& Baker, N. R. 1998. Relationship between $\mathrm{CO}_{2}$ assimilation, photosynthetic electron transport, and active $\mathrm{O}_{2}$ metabolism in leaves of maize in the field during periods of low temperature. Plant Physiol 116:571-80.

Fukuzawa, K. 2000. Singlet oxygen scavenging in phospholipid membranes. Methods Enzymol 319:101-10.

Gardner, T. A., Côté, I. M., Gill, J. A., Grant, A. \& Watkinson, A. R. 2003. Longterm region-wide declines in Caribbean corals. Science 301:958-60.

Gates, R. D., Baghdasarian, G. \& Muscatine, L. 1992. Temperature stress causes host cell detachment in symbiotic cnidarians: Implications for coral bleaching. Biol Bull 182:324-32.

Gates, R. D. \& Edmunds, P. J. 1999. The physiological mechanisms of acclimatization in tropical reef corals. Am Zool 39:30-43.

Gattuso, J. P., Allemand, D. \& Frankignoulle, M. 1999. Photosynthesis and calcification at cellular, organismal and community levels in coral reefs: a review on interactions and control by carbonate chemistry. Am Zool 39:160-83.

Gerschman, R., Gilbert, D. L., Nye, S. W., Dwyer, P. \& Fenn, W. O. 1954. Oxygen poisoning and X-irradiation: A mechanism in common. Science 119:623-26.

Gilmour, J. P., Smith, L. D., Heyward, A. J., Baird, A. H. \& Pratchett, M. S. 2013. Recovery of an isolated coral reef system following severe disturbance. Science 340:69-71.

Glynn, P. W. 1993. Coral reef bleaching: ecological perspectives. Coral Reefs 12:1-17.

Glynn, P. W. 1996. Coral reef bleaching: facts, hypotheses and implications. Glob Change Bio/ 2:495-509. 
Golbuu, Y., Victor, S., Penland, L., Idip Jr, D., Emaurois, C., Okaji, K., Yukihira, H., Iwase, A. \& Van Woesik, R. 2007. Palau's coral reefs show differential habitat recovery following the 1998-bleaching event. Coral Reefs 26:31932.

Gorbunov, M. Y., Kolber, Z. S., Lesser, M. P. \& Falkowski, P. G. 2001. Photosynthesis and photoprotection in symbiotic corals. Limnol Oceanogr 46:75-85.

Grottoli, A. G., Rodrigues, L. J. \& Juarez, C. 2004. Lipids and stable carbon isotopes in two species of Hawaiian corals, Porites compressa and Montipora verrucosa, following a bleaching event. Mar Biol 145:621-31.

Grottoli, A. G., Rodrigues, L. J. \& Palardy, J. E. 2006. Heterotrophic plasticity and resilience in bleached corals. Nature 440:1186-89.

Guillard, R. R. L. 1973. Division rates. In: Stein, J. R. [Ed.] Handbook of Phycological Methods - Culture Methods and Growth Measurements. Cambridge University Press, New York, pp. 298-311.

Guillard, R. R. L. \& Ryther, J. H. 1962. Studies of marine planktonic diatoms. I. Cyclotella nana Hustedt, and Detonula confervacea (cleve) Gran. Can J Microbiol 8:229-39.

Guindon, S. \& Gascuel, O. 2003. A simple, fast and accurate algorithm to estimate large phylogenies by maximum likelihood. Syst Biol 52:696-704.

Guo, J., Lao, Y. \& Chang, D. C. 2009. Calcium and apoptosis. In: Mikoshiba, K. [Ed.] Handbook of Neurochemistry and Molecular Neurobiology: Neural Signaling Mechanisms. Springer Verlag, New York, pp. 597-622.

Guo, R. \& Ki, J. S. 2013. Characterization of a novel catalase-peroxidase (KatG) gene from the dinoflagellate Prorocentrum minimum. J Phycol.

Gutteridge, A. \& Thornton, J. M. 2005. Understanding nature's catalytic toolkit. Trends Biochem Sci 30:622-29.

Habig, W. H., Pabst, M. J. \& Jakoby, W. B. 1974. Glutathione-S-transferase. The first enzymatic step in mercapturic formation. J Biol Chem 249:7130-39.

Halliwell, B. 1984. Chloroplast metabolism. Oxford University Press, Oxford, UK,

Halliwell, B. 1987. Oxidative damage, lipid peroxidation and antioxidant protection in chloroplasts. Chem Phys Lipids 44:327-40.

Halliwell, B. \& Gutteridge, J. M. C. 2007. Free radicals in biology and medicine. 4th edition ed. Oxford University Press, Oxford, UK.

Hancock, J. T. 2008. The role of redox in signal transduction. Methods Mol Biol 476:1-9.

Haugaard, N. 1968. Cellular mechanisms of oxygen toxicity. Physiol Rev 48:31173.

Hawkins, T. D., Bradley, B. J. \& Davy, S. K. 2013. Nitric oxide mediates coral bleaching through an apoptotic-like cell death pathway: evidence from a model sea anemone-dinoflagellate symbiosis. FASEB J.

Hawkins, T. D. \& Davy, S. K. 2012. Nitric oxide production and tolerance differ among Symbiodinium types exposed to heat stress. Plant Cell Physiol 53:1889-98. 
Hawkins, T. D. \& Davy, S. K. 2013. Nitric oxide and coral bleaching: is peroxynitrite generation required for symbiosis collapse? J Exp Biol.

Hawkins, T. D., Krueger, T., Becker, S., Fisher, P. L. \& Davy, S. K. 2014. Differential nitric oxide synthesis and host cell apoptosis correlate with bleaching susceptibility in reef corals. Coral Reefs 33:141-53.

Hernawan, U. E. 2008. Review: Symbiosis between the giant clams (Bivalvia: Cardiidae) and zooxanthellae (Dinophycea). Biodiversitas 9:53-58.

Higuchi, T., Fujimura, H., Arakaki, T. \& Oomori, T. 2008. Activities of antioxidant enzymes (SOD and CAT) in the coral Galaxea fascicularis against increased hydrogen peroxide concentrations in seawater. Proceedings of the 11th International Coral Reef Symposium. Fort Lauderdale, FL, USA, 7-11 July 2008, pp. 926-30.

Higuchi, T., Fujimura, H., Hitomi, Y., Arakaki, T., Oomori, T. \& Suzuki, Y. 2010. Photochemical formation of hydroxyl radicals in tissue extracts of the coral Galaxea fascicularis. Photochem Photobiol 86:1421-26.

Higuchi, T., Suzuki, Y. \& Fujimura, H. 2012. Multiple effects of hydrogen peroxide and temperature on antioxidants and bleaching. Proceedings of the 12th International Coral Reef Symposium. Cairns, Australia, 9-13 July 2012.

Hill, R. \& Ralph, P. J. 2006. Photosystem II heterogeneity of in hospite zooxanthellae in scleractinian corals exposed to bleaching conditions. Photochem Photobio/ 82:1577-85.

Hill, R. \& Ralph, P. J. 2008. Impact of bleaching stress on the function of the oxygen evolving complex of zooxanthellae from scleractinian corals. $J$ Phycol 44:299-310.

Hillis, D. M. \& Bull, J. J. 1993. An empirical test of bootstrapping as a method for assessing confidence in phylogenetic analysis. Syst Biol 42:182-92.

Hinde, R. 1988. Factors produced by symbiotic marine invertebrates which affect translocation between the symbionts. In: Scannerini, S., Smith, D., Bonfante-Fasolo, P. \& Gianinazzi-Pearson, V. [Eds.] Cell to cell signals in plant, animal and microbial symbiosis. Springer-Verlag, Berlin.

Hoegh-Guldberg, O. 1999. Climate change, coral bleaching and the future of the world's coral reefs. Mar Freshw Res 50:839-66.

Hoegh-Guldberg, O. 2012. The adaptation of coral reefs to climate change: Is the Red Queen being outpaced? Sci Mar 76:403-08.

Hoegh-Guldberg, O. \& Jones, R. J. 1999. Photoinhibition and photoprotection in symbiotic dinoflagellates from reef-building corals. Mar Ecol Prog Ser 183:73-86.

Hoegh-Guldberg, O., Mumby, P. J., Hooten, A. J., Steneck, R. S., Greenfield, P., Gomez, E., Harvell, C. D., Sale, P. F., Edwards, A. J., Caldeira, K., Knowlton, N., Eakin, C. M., Iglesias-Prieto, R., Muthiga, N., Bradbury, R. H., Dubi, A. \& Hatziolos, M. E. 2007. Coral reefs under rapid climate change and ocean acidification. Science 318:1737-42.

Hotchkiss, R. S., Strasser, A., McDunn, J. E. \& Swanson, P. E. 2009. Cell Death. N Engl J Med 361:1570. 
Huang, S.-P., Lin, K.-L. \& Fang, L.-S. 1998. The involvement of calcium in heatinduced coral bleaching. Zool Stud 37:89-94.

Hughes, T. P., Baird, A. H., Bellwood, D. R., Card, M., Connolly, S. R., Folke, C., Grosberg, R., Hoegh-Guldberg, O., Jackson, J. B. C., Kleypas, J., Lough, J. M., Marshall, P., Nystrom, M., Palumbi, S. R., Pandolfi, J. M., Rosen, B. \& Roughgarden, J. 2003. Climate change, human impacts, and the resilience of coral reefs. Science 301:929-33.

Iglesias-Prieto, R., Matta, J. L., Robins, W. A. \& Trench, R. K. 1992. Photosynthetic response to elevated temperature in the symbiotic dinoflagellate Symbiodinium microadriaticum in culture. Proc Natl Acad Sci U S A 89:10302-05.

Iglesias-Prieto, R. \& Trench, R. K. 1994. Acclimation and adaptation to irradiance in symbiotic dinoflagellates. I. Responses of the photosynthetic unit to changes in photon flux density. Mar Ecol Prog Ser 113:163-75.

Imbs, A. \& Yakovleva, I. 2012. Dynamics of lipid and fatty acid composition of shallow-water corals under thermal stress: an experimental approach. Coral Reefs 31:41-53.

IPCC 2007. Climate Change 2007: Synthesis Report. Contribution of Working Groups I, II and III to the Fourth Assessment Report of the Intergovernmental Panel on Climate Change. In: Core Writing Team, Pachauri, R. K. \& Reisinger, A. [Eds.]. IPCC, Geneva, Switzerland, pp. 104.

Jennings, D. B., Ehrenshaft, M., Pharr, D. M. \& Williamson, J. D. 1998. Roles for mannitol and mannitol dehydrogenase in active oxygen-mediated plant defense. Proc Natl Acad Sci U S A 95:15129-33.

Jimbo, M., Yanohara, T., Koike, K., Koike, K., Sakai, R., Muramoto, K. \& Kamiya, H. 2000. The d-galactose-binding lectin of the octocoral Sinularia lochmodes: characterization and possible relationship to the symbiotic dinoflagellates. Comp Biochem Physiol B Biochem Mol Biol 125:227-36.

Jokiel, P. \& Coles, S. 1990. Response of Hawaiian and other Indo-Pacific reef corals to elevated temperature. Coral Reefs 8:155-62.

Jones, A. \& Berkelmans, R. 2010. Potential costs of acclimatization to a warmer climate: growth of a reef coral with heat tolerant vs. sensitive symbiont types. PLoS ONE 5:e10437.

Jones, C. G., Lawton, J. H. \& Shachak, M. 1994. Organisms as ecosystem engineers. Oikos 69:373-86.

Jones, R. J. 2008. Coral bleaching, bleaching-induced mortality, and the adaptive significance of the bleaching response. Mar Biol 154:65-80.

Jones, R. J., Hoegh-Guldberg, O., Larkum, A. W. D. \& Schreiber, U. 1998. Temperature-induced bleaching of corals begins with impairment of the $\mathrm{CO}_{2}$ fixation mechanism in zooxanthellae. Plant, Cell Environ 21:1219-30.

Kanematsu, S. \& Asada, K. 1994. Superoxide dismutase. In: Fukui, T. \& Soda, K. [Eds.] Molecular Aspects of Enzyme Catalysis. Kodansha Ltd., Tokyo, Japan. 
Karpinski, S., Escobar, C., Karpinska, B., Creissen, G. \& Mullineaux, P. M. 1997. Photosynthetic electron transport regulates the expression of cytosolic ascorbate peroxidase genes in Arabidopsis during excess light stress. The Plant Cell 9:627-40.

Kawaguti, S. 1944. On the physiology of reef corals. VII. Zooxanthella of the reef corals is Gymnodinium sp., Dinoflagellata; its culture in vitro. Palao Tropical Biological Station Studies 2:675-79.

Kazandjian, A., Shepherd, V. A., Rodriguez-Lanetty, M., Nordemeier, W., Larkum, A. W. D. \& Quinnell, R. G. 2008. Isolation of symbiosomes and the symbiosome membrane complex from the zoanthid Zoanthus robustus. Phycologia 47:294-306.

Kleypas, J. A., McManus, J. W. \& Meñez, L. A. B. 1999. Environmental limits to coral reef development: where do we draw the line? Am Zool 39:146-59.

Knowlton, N. \& Jackson, J. B. C. 2008. Shifting baselines, local impacts, and global change on coral reefs. PLoS Biol 6:e54.

Koike, K., Jimbo, M., Sakai, R., Kaeriyama, M., Muramoto, K., Ogata, T., Maruyama, T. \& H., K. 2004. Octocoral chemical signaling selects and controls dinoflagellate symbionts. Biol Bull 207:80-86.

Kolesar, A. M. \& Mitchelmore, C. L. 2003. Oxidative stress at the onset of symbiosis in the coral Fungia scutaria. In: Cox, E. F. \& Lewis, T. D. [Eds.] Molecular Biology of Corals, Results of 2002 Edwin W. Pauley Summer Program in Marine Biology. University of Hawai'i/Hawai'i Institute of Marine Biology, pp. 66-74.

Kopp, C., Pernice, M., Domart-Coulon, I., Djediat, C., Spangenberg, J., Alexander, D., Hignette, M., Meziane, T. \& Meibom, A. 2013. Highly dynamic cellular-level response of symbiotic coral to a sudden increase in environmental nitrogen. mBio 4.

Kortschak, R. D., Samuel, G., Saint, R. \& Miller, D. J. 2003. EST analysis of the cnidarian Acropora millepora reveals extensive gene loss and rapid sequence divergence in the model invertebrates. Curr Biol 13:2190-95.

Kramarsky-Winter, E., Downs, C., Downs, A. \& Loya, Y. 2009. Cellular responses in the coral Stylophora pistillata exposed to eutrophication from fish mariculture. Evol Ecol Res 11:1-21.

Krämer, W. E., Caamaño-Ricken, I., Richter, C. \& Bischof, K. 2012. Dynamic regulation of photoprotection determines thermal tolerance of two phylotypes of Symbiodinium clade A at two photon fluence rates. Photochem Photobiol.

Krieger-Liszkay, A. 2005. Singlet oxygen production in photosynthesis. J Exp Bot 56:337-46.

Kuguru, B., Achituv, Y., Gruber, D. M. \& Tchernov, D. 2010. Photoacclimation mechanisms of corallimorpharians on coral reefs: Photosynthetic parameters of zooxanthellae and host cellular responses to variation in irradiance. Journal of Experimental Marine Biology and Ecology 394:5362. 
Kühl, M., Cohen, Y., Dalsgaard, T., Jørgensen, B. B. \& Revsbech, N. P. 1995. Microenvironment and photosynthesis of zooxanthellae in scleractinian corals studied with microsensors for $\mathrm{O}_{2}, \mathrm{pH}$ and light. Mar Ecol Prog Ser 117:159-72.

Kvennefors, E. C. E., Leggat, W., Hoegh-Guldberg, O., Degnan, B. M. \& Barnes, A. C. 2008. An ancient and variable mannose-binding lectin from the coral Acropora millepora binds both pathogens and symbionts. Dev Comp Immunol 32:1582-92.

Ladner, J. T., Barshis, D. J. \& Palumbi, S. R. 2012. Protein evolution in two cooccurring types of Symbiodinium: an exploration into the genetic basis of thermal tolerance in Symbiodinium clade D. BMC Evol Biol 12:217.

LaJeunesse, T. C. 2001. Investigating the biodiversity, ecology and phylogeny of endosymbiotic dinoflagellates in the genus Symbiodinium using the ITS region: In search of a "species" level marker. J Phycol 37:866-80.

LaJeunesse, T. C. 2005. "Species" radiations of symbiotic dinoflagellates in the Atlantic and Indo-Pacific since the Miocene-Pliocene transition. Mol Biol Evol 22:570-81.

LaJeunesse, T. C., Loh, W. K. W., van Woesik, R., Hoegh-Guldberg, O., Schmidt, G. W. \& Fitt, W. K. 2003. Low symbiont diversity in southern Great Barrier Reef corals, relative to those of the Caribbean. Limnol Oceanogr 48:2046-54.

Lasi, M., Pauly, B., Schmidt, N., Cikala, M., Stiening, B., Käsbauer, T., Zenner, G., Popp, T., Wagner, A. \& Knapp, R. T. 2010. The molecular cell death machinery in the simple cnidarian Hydra includes an expanded caspase family and pro-and anti-apoptotic Bcl-2 proteins. Cell Res 15:269-78.

Leadley, P. 2010. Biodiversity scenarios: Projections of 21st century change in biodiversity, and associated ecosystem services: A technical report for the Global Biodiversity Outlook 3. UNEP/Earthprint, Secretariat of the Convention on Biological Diversity, Montreal, 132.

Leggat, W., Hoegh Guldberg, O., Dove, S. \& Yellowlees, D. 2007. Analysis of an EST library from the dinoflagellate (Symbiodinium sp.) symbiont of reef building corals. J Phycol 43:1010-21.

Leggat, W., Seneca, F., Wasmund, K., Ukani, L., Yellowlees, D. \& Ainsworth, T. D. 2011. Differential responses of the coral host and their algal symbiont to thermal stress. PLOS ONE 6:e26687.

Lesser, M. P. 1996. Elevated temperatures and ultraviolet radiation cause oxidative stress and inhibit photosynthesis in symbiotic dinoflagellates. Limnol Oceanogr 41:271-83.

Lesser, M. P. 1997. Oxidative stress causes coral bleaching during exposure to elevated temperatures. Coral Reefs 16:187-92.

Lesser, M. P. 2006. Oxidative stress in marine environments: Biochemistry and physiological ecology. Annu Rev Physiol 68:253-78. 
Lesser, M. P. 2011. Coral bleaching: causes and mechanisms. In: Dubinksy, Z. \& Stambler, N. [Eds.] Coral reefs: An ecosystem in transition. Springer, pp. 405-19.

Lesser, M. P. \& Farrell, J. H. 2004. Exposure to solar radiation increases damage to both host tissues and algal symbionts of corals during thermal stress. Coral Reefs 23:367-77.

Lesser, M. P. \& Shick, J. M. 1989a. Effects of irradiance and ultraviolet radiation on photoadaptation in the zooxanthellae of Aiptasia pallida: primary production, photoinhibition, and enzymic defenses against oxygen toxicity. Mar Biol 102:243-55.

Lesser, M. P. \& Shick, J. M. 1989b. Photoadaption and defenses against oxygen toxicity in zooxanthellae from natural populations of symbiotic cnidarians. $J$ Exp Mar Biol Ecol 134:129-41.

Lesser, M. P., Stochaj, W. R., Tapley, D. W. \& Shick, J. M. 1990. Bleaching in coral reef anthozoans: effects of irradiance, ultraviolet radiation, and temperature on the activities of protective enzymes against active oxygen. Coral Reefs 8:225-32.

Levy, O., Achituv, Y., Yacobi, Y. Z., Stambler, N. \& Dubinsky, Z. 2006. The impact of spectral composition and light periodicity on the activity of two antioxidant enzymes (SOD and CAT) in the coral Favia favus. J Exp Mar Biol Ecol 328:35-46.

Lewis, C. L. \& Coffroth, M. A. 2004. The acquisition of exogenous algal symbionts by an octocoral after bleaching. Science 304:1490-92.

Li, Y. \& Goodwin, D. C. 2004. Vital roles of an interhelical insertion in catalaseperoxidase bifunctionality. Biochem Biophys Res Commun 318:970-76.

Lin, K. L., Wang, J. T. \& Fang, L. S. 2000. Participation of glycoproteins on zooxanthellal cell walls in the establishment of a symbiotic relationship with the sea anemone Aiptasia pulchella. Zool Stud 39:172-78.

Liñán-Cabello, M. A., Flores-Ramírez, L. A., Zenteno-Savin, T., Olguín-Monroy, N. O., Sosa-Avalos, R., Patiño-Barragan, M. \& Olivos-Ortiz, A. 2010. Seasonal changes of antioxidant and oxidative parameters in the coral Pocillopora capitata on the Pacific coast of Mexico. Mar Eco/31:407-17.

Little, A. F., van Oppen, M. J. H. \& Willis, B. L. 2004. Flexibility in algal endosymbiosis shapes growth in reef corals. Science 304:1492-94.

Lobban, C. S., Schefter, M., Simpson, A. G. B., Pochon, X., Pawlowski, J. \& Foissner, W. 2002. Maristentor dinoferus n. gen., n. sp., a giant heterotrich ciliate (Spirotrichea: Heterotrichida) with zooxanthellae, from coral reefs on Guam, Marina Islands. Mar Biol 140:411-23.

Logan, D. D. K., LaFlamme, A. C., Weis, V. M. \& Davy, S. K. 2010. Flowcytometric characterization of the cell-surface glycans of symbiotic dinoflagellates (Symbiodinium spp.). J Phycol 46:525-33.

Loram, J. E., Trapido-Rosenthal, H. G. \& Douglas, A. E. 2007. Functional significance of genetically different symbiotic algae Symbiodinium in a coral reef symbiosis. Mol Ecol 16:4849-57. 
Lough, J. \& Barnes, D. 2000. Environmental controls on growth of the massive coral Porites. J Exp Mar Biol Ecol 245:225-43.

Loya, Y., Sakai, K., Yamazato, K., Nakano, Y., Sambali, H. \& van Woesik, R. 2001. Coral bleaching: the winners and the losers. Ecol Lett 4:122-31.

Marshall, P. \& Baird, A. 2000. Bleaching of corals on the Great Barrier Reef: differential susceptibilities among taxa. Coral Reefs 19:155-63.

Martínez, M., Intralawan, A., Vázquez, G., Pérez-Maqueo, O., Sutton, P. \& Landgrave, R. 2007. The coasts of our world: Ecological, economic and social importance. Ecol Econ 63:254-72.

Matta, J. L., Govind, N. S. \& Trench, R. K. 1992. Polyclonal antibodies against iron-superoxide dismutase from Escherichia coli B cross-react with superoxide dismutase from Symbiodinium microadriaticum (Dinophycea). J Phycol 28:343-46.

Maxwell, K. \& Johnson, G. N. 2000. Chlorophyll fluorescence - a practical guide. $J$ Exp Bot 51:659-68.

Mayfield, A. B., Wang, L.-H., Tang, P.-C., Fan, T.-Y., Hsiao, Y.-Y., Tsai, C.-L. \& Chen, C.-S. 2011. Assessing the impacts of experimentally elevated temperature on the biological composition and molecular chaperone gene expression of a reef coral. PLOS ONE 6:e26529.

McGinty, E. S., Pieczonka, J. \& Mydlarz, L. D. 2012. Variations in reactive oxygen release and antioxidant activity in multiple Symbiodinium types in response to elevated temperature. Microb Ecol 64:1000-07.

McLaughlin, J. J. A. \& Zahl, P. A. 1957. Studies in marine biology. II. In vitro culture of zooxanthellae. Proc Soc Exp Biol Med 95:115-21.

McLaughlin, J. J. A. \& Zahl, P. A. 1959. Axenic zooxanthellae from various invertebrate hosts. Ann N Y Acad Sci 77:55-72.

Mehler, A. H. 1951. Studies on reactions of illuminated chloroplasts. I. Mechanisms of the reduction of oxygen and other Hill reagents. Arch Biochem Biophys 33:65-77.

Meyer, E., Aglyamova, G. \& Matz, M. 2011. Profiling gene expression responses of coral larvae (Acropora millepora) to elevated temperature and settlement inducers using a novel RNA-Seq procedure. Mol Ecol 20:3599616.

Middlebrook, R., Anthony, K. R., Hoegh-Guldberg, O. \& Dove, S. 2010. Heating rate and symbiont productivity are key factors determining thermal stress in the reef-building coral Acropora formosa. J Exp Biol 213:1026-34.

Middlebrook, R., Hoegh-Guldberg, O. \& Leggat, W. 2008. The effect of thermal history on the susceptibility of reef-building corals to thermal stress. $J$ Exp Biol 211:1050-6.

Miller, D. J., Hemmrich, G., Ball, E. E., Hayward, D. C., Khalturin, K., Funayama, N., Agata, K. \& Bosch, T. 2007. The innate immune repertoire in Cnidariaancestral complexity and stochastic gene loss. Genome Biol 8:R59. 
Miller, J. R. 2009. Effects of temperature on dimethylsulfoniopropionate concentration in Symbiodinium (Freudenthal 1962). Master thesis, College of Charleston.

Milos, P., Morse, D. \& Hastings, J. 1990. Circadian control over synthesis of many Gonyaulax proteins is at a translational level. Naturwissenschaften 77:87-89.

Misra, H. P. \& Fridovich, I. 1971. The generation of superoxide radical during the autoxidation of ferredoxin. J Biol Chem 246:6886-90.

Mitchelmore, C. L., Ringwood, A. H. \& Weis, V. M. 2003. Differential accumulation of cadmium and changes in glutathione levels as a function of symbiotic state in the sea anemone Anthopleura elegantissima. $J$ Exp Mar Biol Ecol 284:71-85.

Mittler, R., Vanderauwera, S., Gollery, M. \& Van Breusegem, F. 2004. Reactive oxygen gene network of plants. Trends in Plant Science 9:490-98.

Mittler, R., Vanderauwera, S., Suzuki, N., Miller, G., Tognetti, V. B., Vandepoele, K., Gollery, M., Shulaev, V. \& Van Breusegem, F. 2011. ROS signaling: the new wave? Trends in Plant Science 16:300-09.

Møller, I. M., Jensen, P. E. \& Hansson, A. 2007. Oxidative modifications to cellular components in plants. Annu. Rev. Plant Physiol. Plant Mol. Biol. 58:459-81.

Muller-Parker, G. \& D'Elia, C. F. 1997. Interactions between corals and their symbiotic algae. In: Birkeland, C. [Ed.] Life and death of coral reefs. Chapman \& Hall, New York, NY, USA, pp. 96-113.

Mullineaux, P. M. \& Baker, N. R. 2010. Oxidative stress: antagonistic signaling for acclimation or cell death? Plant Physiol 154:521-25.

Mullineaux, P. M., Karpinski, S. \& Creissen, G. P. 2006. Integration of signaling in antioxidant defenses. In: Demmig-Adams, B., Adams III, W. W. \& Mattoo, A. K. [Eds.] Photoprotection, Photoinhibition, Gene Regulation, and Environment. Springer, pp. 223-39.

Munné-Bosch, S. 2005. The role of $\alpha$-tocopherol in plant stress tolerance. J Plant Physiol 162:743-48.

Muscatine, L. \& Cernichiari, E. 1969. Assimilation of photosynthetic products of zooxanthellae by a reef coral. Biol Bull 137:506-23.

Muscatine, L. \& Hand, C. 1958. Direct evidence for the transfer of materials from symbiotic algae to the tissues of a coelenterate. Proc Natl Acad Sci U S A 44:1259.

Muscatine, L. \& Porter, J. W. 1977. Reef corals: mutualistic symbioses adapted to nutrient-poor environments. Bioscience 27:454-60.

Mydlarz, L. D. \& Harvell, C. D. 2007. Peroxidase activity and inducibility in the sea fan coral exposed to a fungal pathogen. Comp Biochem Physiol A Mol Integr Physiol 146:54-62.

Mydlarz, L. D. \& Jacobs, R. S. 2004. Comparison of an inducible oxidative burst in free-living and symbiotic dinoflagellates reveals properties of the pseudopterosins. Phytochemistry 65:3231-41. 
Nakagawa, T. \& Yuan, J. 2000. Cross-talk between two cysteine protease families: activation of caspase-12 by calpain in apoptosis. J Cell Biol 150:887-94.

Nakano, Y. \& Asada, K. 1981. Hydrogen peroxide is scavenged by ascorbatespecific peroxidase in spinach chloroplasts. Plant Cell Physiol 22:867-80.

Navrot, N., Collin, V., Gualberto, J., Gelhaye, E., Hirasawa, M., Rey, P., Knaff, D. B., Issakidis, E., Jacquot, J.-P. \& Rouhier, N. 2006. Plant glutathione peroxidases are functional peroxiredoxins distributed in several subcellular compartments and regulated during biotic and abiotic stresses. Plant Physiol 142:1364-79.

Navrot, N., Rouhier, N., Gelhaye, E. \& Jacquot, J. P. 2007. Reactive oxygen species generation and antioxidant systems in plant mitochondria. Physiol Plant 129:185-95.

Nii, C. M. \& Muscatine, L. 1997. Oxidative stress in the symbiotic sea anemone Aiptasia pulchella (Carlgren, 1943): contribution of the animal to superoxide ion production at elevated temperature. Biol Bull 192:444-56.

Noctor, G., Gomez, L., Vanacker, H. \& Foyer, C. H. 2002. Interactions between biosynthesis, compartmentation and transport in the control of glutathione homeostasis and signalling. $J$ Exp Bot 53:1283-304.

Nowak, M. A., Boerlijst, M. C., Cooke, J. \& Smith, J. M. 1997. Evolution of genetic redundancy. Nature 388:167-71.

Nyholm, S. V. \& McFall-Ngai, M. J. 2004. The winnowing: establishing the squidVibrio symbiosis. Nature Rev Microbio/ 2:632-42.

Oberst, A., Bender, C. \& Green, D. R. 2008. Living with death: the evolution of the mitochondrial pathway of apoptosis in animals. Cell Death Differ 15:1139-46.

Okamoto, O. K., Robertson, D. L., Fagan, T. F., Hastings, J. W. \& Colepicolo, P. 2001. Different regulatory mechanisms modulate the expression of a dinoflagellate iron-superoxide dismutase. J Biol Chem 276:19989-93.

Oliver, J., Berkelmans, R. \& Eakin, C. 2009. Coral bleaching in space and time. Coral bleaching. Springer, pp. 21-39.

Osaka, T., Beika, A., Hattori, A., Kohno, Y., Kato, K. H. \& Mizutani, T. 2003. The protozoa dinoflagellate Oxyrrhis marina contains selenoproteins and the relevant translation apparatus. Biochem Biophys Res Commun 300:23640.

Ouyang, X., Tran, Q. T., Goodwin, S., Wible, R. S., Sutter C.H. \& T.R., S. 2011. Yap1 activation by $\mathrm{H}_{2} \mathrm{O}_{2}$ or thiol-reactive chemicals elicits distinct adaptive gene responses. Free Radical Biol Med 50:1-13.

Palenik, B. \& Morel, F. M. 1990. Comparison of cell-surface L-amino acid oxidases from several marine phytoplankton. Mar Ecol Prog Ser 59:195201. 
Pandolfi, J. M., Bradbury, R. H., Sala, E., Hughes, T. P., Bjorndal, K. A., Cooke, R. G., McArdle, D., McClenachan, L., Newman, M. J. H. \& Paredes, G. 2003. Global trajectories of the long-term decline of coral reef ecosystems. Science 301:955-58.

Paxton, C. W., Davy, S. K. \& Weis, V. M. 2013. Stress and death of cnidarian host cells play a role in cnidarian bleaching. J Exp Biol.

Pearse, V. B. \& Muscatine, L. 1971. Role of symbiotic algae (zooxanthellae) in coral calcification. Biol Bull 141:350-63.

Peng, S. E., Wang, Y. B., Wang, L. H., Chen, W. N. U., Lu, C. Y., Fang, L. S. \& Chen, C. S. 2010. Proteomic analysis of symbiosome membranes in Cnidaria-dinoflagellate endosymbiosis. Proteomics 10:1002-16.

Perez, S. \& Weis, V. M. 2006. Nitric oxide and cnidarian bleaching: an eviction notice mediates breakdown of a symbiosis. J Exp Biol 209:2804-10.

Pfaffl, M. W., Horgan, G. W. \& Dempfle, L. 2002. Relative expression software tool (REST@) for group-wise comparison and statistical analysis of relative expression results in real-time PCR. Nucleic Acids Res 30:e36-e36.

Pitsch, N. T., Witsch, B. \& Baier, M. 2010. Comparison of the chloroplast peroxidase system in the chlorophyte Chlamydomonas reinhardtii, the bryophyte Physcomitrella patens, the lycophyte Selaginella moellendorffii and the seed plant Arabidopsis thaliana. BMC Plant Biology 10:133.

Pochon, X. \& Gates, R. D. 2010. A new Symbiodinium clade (Dinophyceae) from soritid foraminifera in Hawai'i. Mol Phylogenet Evol 56:492-97.

Pochon, X., Montoya-Burgos, J. I., Stadelmann, B. \& Pawlowski, J. 2006. Molecular phylogeny, evolutionary rates, and divergence timing of the symbiotic dinoflagellate genus Symbiodinium. Mol Phylogenet Evol 38:2030.

Pochon, X., Pawlowski, J., Zaninetti, L. \& Rowan, R. 2001. High genetic diversity and relative specificity among Symbiodinium-like endosymbiotic dinoflagellates in soritid foraminiferans. Mar Biol 139:1069-78.

Pochon, X., Putnam, H. M. \& Gates, R. D. 2014. Multi-gene analysis of the symbiotic and free-living dinoflagellate genus Symbiodinium. PeerJ 10.7287/peerj.preprints.267v1.

Polato, N. R., Voolstra, C. R., Schnetzer, J., DeSalvo, M. K., Randall, C. J., Szmant, A. M., Medina, M. \& Baums, I. B. 2010. Location-specific responses to thermal stress in larvae of the reef-building coral Montastraea faveolata. PLOS ONE 5:e11221.

Porra, R., Thompson, W. \& Kriedemann, P. 1989. Determination of accurate extinction coefficients and simultaneous equations for assaying chlorophylls $a$ and $b$ extracted with four different solvents: verification of the concentration of chlorophyll standards by atomic absorption spectroscopy. Biochim Biophys Acta 975:384-94.

Pratchett, M. S., McCowan, D., Maynard, J. A. \& Heron, S. F. 2013. Changes in bleaching susceptibility among corals subject to ocean warming and recurrent bleaching in Moorea, French Polynesia. PLoS ONE 8:e70443. 
Prézelin, B. B. 1987. Photosynthetic physiology of dinoflagellates. In: Taylor, M. [Ed.] The Biology of Dinoflagellates. Blackwell, New York, USA, pp. 174223.

Putnam, H., Mayfield, A., Fan, T., Chen, C. \& Gates, R. 2012a. The physiological and molecular responses of larvae from the reef-building coral Pocillopora damicornis exposed to near-future increases in temperature and $\mathrm{pCO}_{2}$. Mar Biol 160:2157-73.

Putnam, H. M., Stat, M., Pochon, X. \& Gates, R. D. 2012b. Endosymbiotic flexibility associates with environmental sensitivity in scleractinian corals. Proc $R$ Soc $B$ 279:4352-61

Radi, R., Peluffo, G., Alvarez, M. a. N., Naviliat, M. \& Cayota, A. 2001. Unraveling peroxynitrite formation in biological systems. Free Radical Biol Med 30:463-88.

Ragni, M., Airs, R. L., Hennige, S. J., Suggett, D. J., Warner, M. E. \& Geider, R. J. 2010. PSII photoinhibition and photorepair in Symbiodinium (Pyrrhophyta) differs between thermally tolerant and sensitive phylotypes. Mar Ecol Prog Ser 406:57-70.

Ralph, P. J., Gademann, R. \& Larkum, A. W. D. 2001. Zooxanthellae expelled from bleached corals at $33^{\circ} \mathrm{C}$ are photosynthetically competent. Mar Ecol Prog Ser 220:163-68.

Ramos, R., Bastidas, C., Debrot, D. \& García, E. 2011. Phase I and II biotransformation and antioxidant enzymes in the coral Siderastrea siderea act as biomarkers for reproductive condition and habitat quality. Mar Biol Res 7:398-406.

Ramos, R. \& García, E. 2007. Induction of mixed-function oxygenase system and antioxidant enzymes in the coral Montastraea faveolata on acute exposure to benzo(a)pyrene. Comp Biochem Physiol C Toxicol Pharmacol 144:34855.

Rands, M. L., Loughman, B. C. \& Douglas, A. E. 1993. The symbiotic interface in an algal-invertebrate symbiosis. Proc $R$ Soc $B$ 253:161-65.

Reynolds, W. S., Schwarz, J. A. \& Weis, V. M. 2000. Symbiosis-enhanced gene expression in cnidarian-algal associations: cloning and characterization of a cDNA, sym32, encoding a possible cell adhesion protein. Comp Biochem Physiol A Mol Integr Physiol 126:33-44.

Richier, S., Furla, P., Plantivaux, A., Merle, P.-L. \& Allemand, D. 2005. Symbiosis-induced adaptation to oxidative stress. J Exp Biol 208:277-85.

Richier, S., Merle, P.-L., Furla, P., Pigozzi, D., Sola, F. \& Allemand, D. 2003. Characterization of superoxide dismutases in anoxia- and hyperoxiatolerant symbiotic cnidarians. Biochim Biophys Acta 1621:84-91.

Richier, S., Sabourault, C., Courtiade, J., Zucchini, N., Allemand, D. \& Furla, P. 2006. Oxidative stress and apoptotic events during thermal stress in the symbiotic sea anemone, Anemonia viridis. FEBS J 273:4186-98.

Richter, C. 1987. Biophysical consequences of lipid peroxidation in membranes. Chem Phys Lipids 44:175-89. 
Rijstenbil, J. W. 2002. Assessment of oxidative stress in the planktonic diatom Thallassiosira pseudonana in response to UVA and UVB radiation. $J$ Plankton Res 24:1277-88.

Rizhsky, L., Hallak-Herr, E., Van Breusegem, F., Rachmilevitch, S., Barr, J. E., Rodermel, S., Inzé, D. \& Mittler, R. 2002. Double antisense plants lacking ascorbate peroxidase and catalase are less sensitive to oxidative stress than single antisense plants lacking ascorbate peroxidase or catalase. Plant J 32:329-42.

Roberts, J. M., Wheeler, A. J. \& Freiwald, A. 2006. Reefs of the deep: the biology and geology of cold-water coral ecosystems. Science 312:543-47.

Robison, J. D. \& Warner, M. E. 2006. Differential impacts of photoacclimation and thermal stress on the photobiology of four different phylotypes of Symbiodinium (Pyrrhophyta). J Phycol 42:568-79.

Rockel, P., Strube, F., Rockel, A., Wildt, J. \& Kaiser, W. M. 2002. Regulation of nitric oxide (NO) production by plant nitrate reductase in vivo and in vitro. $J$ Exp Bot 53:103-10.

Rodriguez-Lanetty, M., Harii, S. \& Hoegh-Guldberg, O. 2009. Early molecular responses of coral larvae to hyperthermal stress. Mol Ecol 18:5101-14.

Rosic, N. N., Pernice, M., Dove, S., Dunn, S. \& Hoegh-Guldberg, O. 2011a. Gene expression profiles of cytosolic heat shock proteins Hsp70 and Hsp90 from symbiotic dinoflagellates in response to thermal stress: possible implications for coral bleaching. Cell Stress Chaperones 16:69-80.

Rosic, N. N., Pernice, M., Rodriguez-Lanetty, M. \& Hoegh-Guldberg, O. 2011b. Validation of housekeeping genes for gene expression studies in Symbiodinium exposed to thermal and light stress. Mar Biotechnol 13:35565.

Rowan, R. 2004a. Coral bleaching: Thermal adaptation in reef coral symbionts. Nature 430:742-42.

Rowan, R. 2004b. Thermal adaptations in reef coral symbionts. Nature 430:742.

Rowan, R. \& Knowlton, N. 1995. Intraspecific diversity and ecological zonation in coral-algal symbiosis. Proc Natl Acad Sci U S A 92:2850-53.

Rowan, R., Knowlton, N., Baker, A. \& Jara, J. 1997. Landscape ecology of algal symbionts creates variation in episodes of coral bleaching. Nature 388:265-69.

Rowan, R. \& Powers, D. A. 1991a. A molecular genetic classification of zooxanthellae and the evolution of animal-algal symbioses. Science 251:1348-51.

Rowan, R. \& Powers, D. A. 1991b. Molecular genetic identification of symbiotic dinoflagellates (zooxanthellae). Mar Ecol Prog Ser 71:65-73.

Rowan, R., Whitney, S. M., Fowler, A. \& Yellowlees, D. 1996. Rubisco in marine symbiotic dinoflagellates: form II enzymes in eukaryotic oxygenic phototrophs encoded by a nuclear multigene family. The Plant Cell 8:53953. 
Roy, A., Kucukural, A. \& Zhang, Y. 2010. I-TASSER: a unified platform for automated protein structure and function prediction. Nat Protoc 5:725-38.

Safavi-Hemami, H., Young, N. D., Doyle, J., Llewellyn, L., Klueter, A. \& Stadler, K. 2010. Characterisation of nitric oxide synthase in three cnidariandinoflagellate symbioses. PLOS ONE 5:e10379.

Salamov, A. A., Nishikawa, T. \& Swindells, M. B. 1998. Assessing protein coding region integrity in cDNA sequencing projects. Bioinformatics 14:384-90.

Sampayo, E. M., Dove, S. \& LaJeunesse, T. C. 2009. Cohesive molecular genetic data delineate species diversity in the dinoflagellate genus Symbiodinium. Mol Ecol 18:500-19.

Sampayo, E. M., Ridgway, T., Bongaerts, P. \& Hoegh-Guldberg, O. 2008. Bleaching susceptibility and mortality of corals are determined by finescale differences in symbiont type. Proc Natl Acad Sci U S A 105:1044449.

Sandeman, I. M. 2006. Fragmentation of the gastrodermis and detachment of zooxanthellae in symbiotic cnidarians: a role for hydrogen peroxide and $\mathrm{Ca}^{2+}$ in coral bleaching and algal density control. Rev Biol Trop 54:79-96.

Santos, S. R., Taylor, D. J., Kinzie III, R. A., Hidaka, M., Sakai, K. \& Coffroth, M. A. 2002. Molecular phylogeny of symbiotic dinoflagellates inferred from partial chloroplast large subunit (23S)-rDNA sequences. Mol Phylogenet Evol 23:97-111.

Saragosti, E., Tchernov, D., Katsir, A. \& Shaked, Y. 2010. Extracellular production and degradation of superoxide in the coral Stylophora pistillata and cultured Symbiodinium. PLOS ONE 5:e12508.

Sawyer, S. J. \& Muscatine, L. 2001. Cellular mechanisms underlying temperature-induced bleaching in the tropical sea anemone Aiptasia pulchella. J Exp Biol 204:3443-56.

Schoenberg, D. A. \& Trench, R. K. 1980a. Genetic variation in Symbiodinium (=Gymnodinium) microadriaticum Freudenthal, and specificity in its symbiosis with marine invertebrates. I. Isoenzyme and soluble protein patterns of axenic cultures of Symbiodinium microadriaticum. Proc $R$ Soc B 207:405-27.

Schoenberg, D. A. \& Trench, R. K. 1980b. Genetic variation in Symbiodinium (=Gymnodinium) microadriaticum Freudenthal, and specificity in its symbiosis with marine invertebrates. II. Morphological variation in Symbiodinium microadriaticum. Proc $R$ Soc B 207:429-44.

Schwarz, J. A., Brokstein, P. B., Voolstra, C., Terry, A. Y., Miller, D. J., Szmant, A. M., Coffroth, M. A. \& Medina, M. 2008. Coral life history and symbiosis: functional genomic resources for two reef building Caribbean corals, Acropora palmata and Montastraea faveolata. BMC Genomics 9:97.

Schwarz, J. A. \& Weis, V. M. 2003. Localization of a symbiosis-related protein, Sym32, in the Anthopleura elegantissima-Symbiodinium muscatinei association. Biol Bull 205:339-50. 
Shaik, I. H. \& Mehvar, R. 2006. Rapid determination of reduced and oxidized glutathione levels using a new thiol-masking reagent and the enzymatic recycling method: application to the rat liver and bile samples. Anal Bioanal Chem 385:105-13.

Sharp, V. A., Brown, B. E. \& Miller, D. 1997. Heat shock protein (hsp 70) expression in the tropical reef coral Goniopora djiboutiensis. J Therm Biol 22:11-19.

Shashar, N., Cohen, Y. \& Loya, Y. 1993. Extreme diel fluctuations of oxygen in diffusive boundary layers surrounding stony corals. Biol Bull 185:455-61.

Shen, B., Jensen, R. G. \& Bohnert, H. J. 1997. Mannitol protects against oxidation by hydroxyl radicals. Plant Physiol 115:527-32.

Sheppard, C. R. C., Davy, S. K. \& Pilling, G. M. 2009. The biology of coral reefs. Oxford University Press, New York, USA,

Sheptovitsky, Y., G. \& Brudvig, G. W. 1998. Catalase-free photosystem II: The $\mathrm{O}_{2}$-evolving complex does not dismutate hydrogen peroxide. Biochemistry 37:5052-59.

Shick, J. M., Lesser, M. P., Dunlap, W. C., Stochaj, W. R., Chalker, B. E. \& Wu Won, J. 1995. Depth-dependent responses to solar ultraviolet radiation and oxidative stress in the zooxanthellae coral Acropora microphthalma. Mar Biol 122:41-51.

Shinzato, C., Shoguchi, E., Kawashima, T., Hamada, M., Hisata, K., Tanaka, M., Fujie, M., Fujiwara, M., Koyanagi, R. \& Ikuta, T. 2011. Using the Acropora digitifera genome to understand coral responses to environmental change. Nature 476:320-23.

Sies, H. 1991. Oxidative stress II. Oxidants and antioxidants. Academic Press, London, UK,

Sies, H. 1999. Glutathione and its role in cellular functions. Free Radical Biol Med 27:916-21.

Silverman, J., Lazar, B. \& Erez, J. 2007. Effect of aragonite saturation, temperature, and nutrients on the community calcification rate of a coral reef. Journal of Geophysical Research: Oceans (1978-2012) 112.

Smith-Keune, C. \& van Oppen, M. 2006. Genetic structure of a reef-building coral from thermally distinct environments on the Great Barrier Reef. Coral Reefs 25:493-502.

Smith, I. K., Vierheller, T. I. \& Thorne, C. A. 1988. Assay of glutathione reductase in crude tissue homogenates using 5,5-Dithiobis(2-nitrobenzoic acid). Anal Biochem 175:408-13.

Smulevich, G., Jakopitsch, C., Droghetti, E. \& Obinger, C. 2006. Probing the structure and bifunctionality of catalase-peroxidase (KatG). J Inorg Biochem 100:568-85.

Stambler, N. 2010. Coral symbiosis under stress. In: Seckbach, J. \& Grube, M. [Eds.] Symbioses and Stress. Springer, Dordrecht, pp. 197-224. 
Stat, M., Carter, D. \& Hoegh-Guldberg, O. 2006. The evolutionary history of Symbiodinium and scleractinian hosts - Symbiosis, diversity, and the effect of climate change. Perspect Plant Ecol Evol Syst 8:26-43.

Stat, M., Loh, W. K. W., Hoegh-Guldberg, O. \& Carter, D. A. 2008a. Symbiont acquisition strategy drives host-symbiont associations in the southern Great Barrier Reef. Coral Reefs 27:763-72.

Stat, M., Loh, W. K. W., LaJeunesse, T. C., Hoegh-Guldberg, O. \& Carter, D. A. 2009. Stability of coral-endosymbiont associations during and after a thermal stress event in the southern Great Barrier Reef. Coral Reefs 28:709-13.

Stat, M., Morris, E. \& Gates, R. D. 2008b. Functional diversity in coraldinoflagellate symbiosis. Proc Natl Acad Sci U S A 105:9256-61.

Steen, R. G. \& Muscatine, L. 1987. Low temperature evokes rapid exocytosis of symbiotic algae by a sea anemone. Biol Bull 172:246-63.

Stimson, J. \& Kinzie, R. A. 1991. The temporal pattern and rate of release of zooxanthellae from the reef coral Pocillopora damicornis (Linnaeus) under nitrogen-enrichment and control conditions. J Exp Mar Biol Ecol 153:6374.

Stimson, J., Sakai, K. \& Sembali, H. 2002. Interspecific comparison of the symbiotic relationship in corals with high and low rates of bleachinginduced mortality. Coral Reefs 21:409-21.

Suggett, D. J., Warner, M. E., Smith, D. J., Davey, P., Hennige, S. \& Baker, N. R. 2008. Photosynthesis and production of hydrogen peroxide by Symbiodinium (Pyrrhophyta) phylotypes with different thermal tolerances. $J$ Phycol 44:948-56.

Sumimoto, H. 2008. Structure, regulation and evolution of Nox-family NADPH oxidases that produce reactive oxygen species. FEBS $J$ 275:3249-77.

Sunagawa, S., Choi, J., Forman, H. J. \& Medina, M. 2008. Hyperthermic stressinduced increase in the expression of glutamate-cysteine ligase and glutathione levels in the symbiotic sea anemone Aiptasia pallida. Comp Biochem Physiol B Biochem Mol Biol 151:133-38.

Sunda, W., Kieber, D. J., Kiene, R. P. \& Huntsman, S. 2002. An antioxidant function for DMSP and DMS in marine algae. Nature 418:317-20.

Tachibana, T., Okazaki, S., Murayama, A., Naganuma, A., Nomoto, A. \& Kuge, S. 2009. A major peroxiredoxin-induced activation of Yap1 transcription factor is mediated by reduction-sensitive disulfide bonds and reveals a low level of transcriptional activation. J Biol Chem 284:4464-72.

Taguchi, S. \& Kinzie III, R. A. 2001. Growth of zooxanthellae in culture with two nitrogen sources. Mar Biol 138:149-55.

Takabayashi, M., Santos, S. R. \& Cook, C. B. 2004. Mitochondrial DNA phylogeny of the symbiotic dinoflagellates (Symbiodinium, Dinophyta). $J$ Phycol 40:160-64. 
Takahashi, M.-A. \& Asada, K. 1983. Superoxide anion permeability of phospholipid membranes and chloroplast thylakoids. Arch Biochem Biophys 226:558-66.

Takahashi, S., Nakamura, T., Sakamizu, M., van Woesik, R. \& Yamasaki, H. 2004. Repair machinery of symbiotic photosynthesis as the primary target of heat stress for reef-building corals. Plant Cell Physiol 45:251-55.

Takahashi, S., Whitney, S., Itoh, S., Maruyama, T. \& Badger, M. 2008. Heat stress causes inhibition of the de novo synthesis of antenna proteins and photobleaching in cultured Symbiodinium. Proc Natl Acad Sci U S A 105:4203-08.

Takahashi, S., Whitney, S. M. \& Badger, M. R. 2009. Different thermal sensitivity of the repair of photodamaged photosynthetic machinery in cultured Symbiodinium species. Proc Natl Acad Sci U S A 106:3237-42.

Takishita, K., Ishida, K.-i. \& Maruyama, T. 2003a. An enigmatic GAPDH Gene in the symbiotic dinoflagellate genus Symbiodinium and its related species (the order Suessiales): Possible lateral gene transfer between two eukaryotic algae, dinoflagellate and euglenophyte. Protist 154:443-54.

Takishita, K., Ishikura, M. \& Koike, M., T. 2003b. Comparison of phylogenies based on nuclear-encoded SSU rDNA and plastid-encoded psbA in the symbiotic dinoflagellate genus Symbiodinium. Phycologia 42:285-91.

Tanaka, Y., Miyajima, T., Koike, I., Hayashibara, T. \& Ogawa, H. 2006. Translocation and conservation of organic nitrogen within the coralzooxanthella symbiotic system of Acropora pulchra, as demonstrated by dual isotope-labeling techniques. J Exp Mar Biol Ecol 336:110-19.

Tchernov, D., Gorbunov, M. Y., de Vargas, C., Yadav, S. N., Milligan, A. J., Häggblom, M. \& Falkowski, P. G. 2004. Membrane lipids of symbiotic algae are diagnostic of sensitivity to thermal bleaching in corals. Proc Natl Acad Sci U S A 101:13531-35.

Tchernov, D., Kvitt, H., Haramaty, L., Bibby, T. S., Gorbunov, M. Y., Rosenfeld, H. \& Falkowski, P. G. 2011. Apoptosis and the selective survival of host animals following thermal bleaching in zooxanthellate corals. Proc Natl Acad Sci U S A 108:9905-09.

Teixeira, T., Diniz, M., Calado, R. \& Rosa, R. 2013. Coral physiological adaptations to air exposure: Heat shock and oxidative stress responses in Veretillum cynomorium. J Exp Mar Biol Ecol 439:35-41.

Thompson, J. D., Gibson, T. J., Plewniak, F., Jeanmougin, F. \& Higgins, D. G. 1997. The ClustalX windows interface: Flexible strategies for multiple sequence alignment aided by quality analysis tools. Nucleic Acids Res 24:4876-82.

Toller, W. W., Rowan, R. \& Knowlton, N. 2001. Repopulation of zooxanthellae in the Caribbean corals Montastraea annularis and M. faveolata following experimental and disease-associated bleaching. Biol Bull 201:360-73. 
Tolleter, D., Seneca, F. O., DeNofrio, J. C., Krediet, C. J., Palumbi, S. R., Pringle, J. R. \& Grossman, A. R. 2013. Coral bleaching independent of photosynthetic activity. Curr Biol 23:1782-86.

Trench, R. K. 1997. Diversity of symbiotic dinoflagellates and the evolution of microalgal-invertebrate symbiosis. Proceedings of the 8th International Coral Reef Symposium. Panama City, Panama, 24-29 July 1997, pp. 1275-86.

Ulstrup, K. E., Van Oppen, M., Kühl, M. \& Ralph, P. J. 2007. Inter-polyp genetic and physiological characterisation of Symbiodinium in an Acropora valida colony. Mar Biol 153:225-34.

Ulstrup, K. E. \& van Oppen, M. J. H. 2003. Geographic and habitat partitioning of genetically distinct zooxanthellae (Symbiodinium) in Acropora corals on the Great Barrier Reef. Mol Ecol 12:3477-84.

Untergasser, A., Cutcutache, I., Koressaar, T., Ye, J., Faircloth, B. C., Remm, M. \& Rozen, S. G. 2012. Primer3 - New capabilities and interfaces. Nucleic Acids Res 40:e115-e15.

van Beukering, P., Haider, W., Longland, M., Cesar, H., Sablan, J., Shjegstad, S., Beardmore, B., Liu, Y. \& Garces, G. O. 2007. The economic value of Guam's coral reefs. University of Guam Marine Laboratory,

van Oppen, M. J. H., Palstra, F. P., Piquet, A. M.-T. \& Miller, D. J. 2001. Patterns of coral-dinoflagellate associations in Acropora: significance of local availability and physiology of Symbiodinium strains and host-symbiont selectivity. Proc $R$ Soc $B$ 268:1759-67.

Venn, A. A., Loram, J. E. \& Douglas, A. E. 2008. Photosynthetic symbioses in animals. J Exp Bot 59:1069-80.

Venn, A. A., Wilson, M. A., Trapido-Rosenthal, H. G., Keely, B. J. \& Douglas, A. E. 2006. The impact of coral bleaching on the pigment profile of the symbiotic algae, Symbiodinium. Plant, Cell Environ 29:2133-42.

Vieira, H. L., Haouzi, D., El Hamel, C., Jacotot, E., Belzacq, A. S., Brenner, C. \& Kroemer, G. 2000. Permeabilization of the mitochondrial inner membrane during apoptosis: impact of the adenine nucleotide translocator. Cell Death Differ 7:1146-54.

Voolstra, C. R., Sunagawa, S., Schwarz, J. A., Coffroth, M. A., Yellowlees, D., Leggat, W. \& Medina, M. 2009. Evolutionary analysis of orthologous cDNA sequences from cultured and symbiotic dinoflagellate symbionts of reefbuilding corals (Dinophyceae: Symbiodinium). Comp Biochem Physiol D Genomics Proteomics 4:67-74.

Wakefield, T. S. \& Kempf, S. C. 2001. Development of host- and symbiontspecific monoclonal antibodies and confirmation of the origin of the symbiosome membrane in a cnidarian-dinoflagellate symbiosis. Biol Bull 200:127-43.

Wang, J.-T. \& Douglas, A. E. 1997. Nutrients, signals, and photosynthate release by symbiotic algae. Plant Physiol 114:631-63. 
Warner, M. E. \& Berry-Lowe, S. 2006. Differential xanthophyll cycling and photochemical activity in symbiotic dinoflagellates in multiple locations of three species of Caribbean coral. J Exp Mar Biol Ecol 339:86-95.

Warner, M. E., Fitt, W. K. \& Schmidt, G. W. 1996. The effects of elevated temperature on the photosynthetic efficiency of zooxanthellae in hospite from four different species of reef coral: A novel approach. Plant, Cell Environ 19:291-99.

Warner, M. E., Fitt, W. K. \& Schmidt, G. W. 1999. Damage to photosystem II in symbiotic dinoflagellates: A determinant of coral bleaching. Proc Natl Acad Sci U S A 96:8007-12.

Weis, V. M. 2008. Cellular mechanisms of cnidarian bleaching: stress causes the collapse of symbiosis. J Exp Biol 211:3059-66.

Weis, V. M., Davy, S. K., Hoegh-Guldberg, O., Rodriguez-Lanetty, M. \& Pringe, J. R. 2008. Cell biology in model systems as the key to understanding corals. Trends Ecol Evol 23:369-76.

Weis, V. M., Reynolds, W. S., deBoer, M. D. \& Krupp, D. A. 2001. Host-symbiont specificity during onset of symbiosis between the dinoflagellates Symbiodinium spp. and planula larvae of the scleractinian coral Fungia scutaria. Coral Reefs 20:301-08.

Welinder, K. G. 1991. Bacterial catalase-peroxidases are gene duplicated members of the plant peroxidase superfamily. Biochim Biophys Acta 1080:215-20.

Whitehead, L. F. \& Douglas, A. E. 2003. Metabolite comparisons and the identity of nutrients translocated from symbiotic algae to an animal host. $J$ Exp Biol 206:3149-57.

Wilkinson, C. 2008. Status of coral reefs of the world. Australian Institute of Marine Science, Townsville, Australia,

Willekens, H., Chamnongpol, S., Davey, M., Schraudner, M., Langebartels, C., Van Montagu, M., Inzé, D. \& Van Camp, W. 1997. Catalase is a sink for $\mathrm{H}_{2} \mathrm{O}_{2}$ and is indispensable for stress defence in $\mathrm{C} 3$ plants. EMBO $\mathrm{J}$ 16:4806-16.

Wink, D. A., Hines, H. B., Cheng, R. Y., Switzer, C. H., Flores-Santana, W., Vitek, M. P., Ridnour, L. A. \& Colton, C. A. 2011. Nitric oxide and redox mechanisms in the immune response. J Leukocyte Biol 89:873-91.

Winterbourn, C. C. 2008. Reconciling the chemistry and biology of reactive oxygen species. Nat Chem Biol 4:278-86.

Wintjens, R., Gilis, D. \& Rooman, M. 2008. Mn/Fe superoxide dismutase interaction fingerprints and prediction of oligomerization and metal cofactor from sequence. Proteins Structure Function and Bioinformatics 70:156477.

Wisecaver, J. H. \& Hackett, J. D. 2011. Dinoflagellate genome evolution. Annu Rev Microbio/ 65:369-87. 
Wood-Charlson, E. M., Hollingsworth, L. L., Krupp, D. A. \& Weis, V. M. 2006. Lectin/glycan interactions play a role in recognition in a coral/dinoflagellate symbiosis. Cell Microbiol 8:1985-93.

Yakovleva, I., Bhagooli, R., Takemura, A. \& Hidaka, M. 2004. Differential susceptibility to oxidative stress of two scleractinian corals: antioxidant functioning of mycrosporine-glycine. Comp Biochem Physiol B Biochem Mol Biol 139:721-30.

Yakovleva, I. M., Baird, A. H., Yamamoto, H. H., Bhagooli, R., Nonaka, M. \& Hidaka, M. 2009. Algal symbionts increase oxidative damage and death in coral larvae at high temperatures. Mar Ecol Prog Ser 378:105-12.

Yellowlees, D., Rees, T. A. V. \& Leggat, W. 2008. Metabolic interactions between algal symbionts and invertebrate hosts. Plant, Cell Environ 31:679-94.

Yost, D. M. \& Mitchelmore, C. L. 2009. Dimethylsulfoniopropionate (DMSP) lyase activity in different strains of the symbiotic alga Symbiodinium microadriaticum. Mar Ecol Prog Ser 386:61-70.

Youle, R. J. \& Strasser, A. 2008. The Bcl-2 protein family: opposing activities that mediate cell death. Nat Rev Mol Cell Biol 9:47-59.

Zámocký, M., Furtmüller, P. G. \& Obinger, C. 2010. Evolution of structure and function of Class I peroxidases. Arch Biochem Biophys 500:45-57.

Zdobnov, E. M. \& Apweiler, R. 2001. InterProScan-an integration platform for the signature-recognition methods in InterPro. Bioinformatics 17:847-48.

Zhang, H. \& Lin, S. 2009. Retrieval of missing spliced leader in dinoflagellates. PLOS ONE 4:e4129.

Zhang, Y. 2008. I-TASSER server for protein 3D structure prediction. BMC Bioinformatics 9:40.

Zmasek, C. M., Zhang, Q., Ye, Y. \& Godzik, A. 2007. Surprising complexity of the ancestral apoptosis network. Genome Biol 8:R226.

Zor, T. \& Selinger, Z. 1996. Linearization of the Bradford protein assay increases its sensitivity: theoretical and experimental studies. Anal Biochem 236:302-08. 
- 144 - 


\section{APPENDIX}

\section{Appendix for Chapter 2}
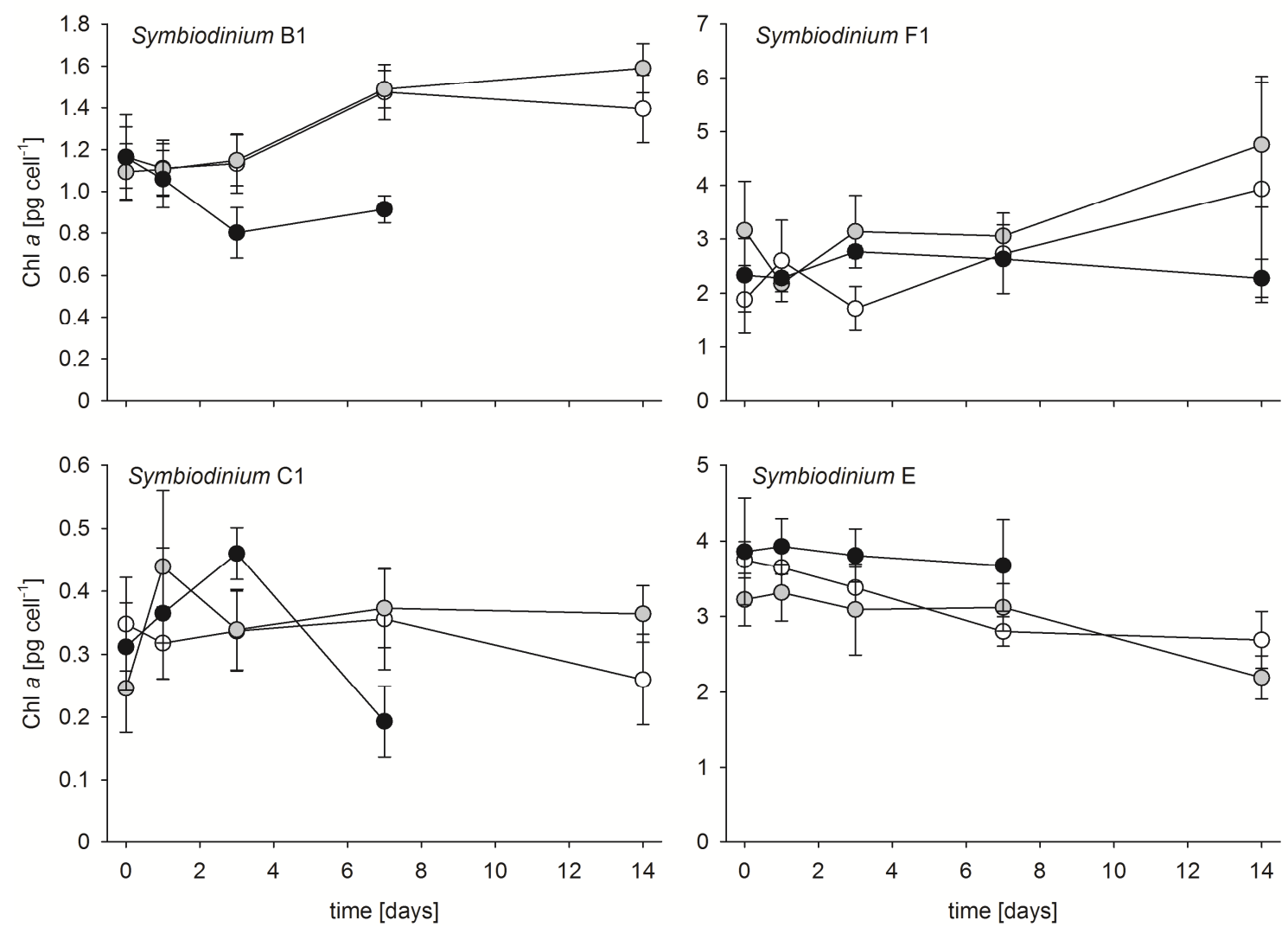

Figure 7.1. Chlorophyll a content per cell.

Chlorophyll a content (pg cell ${ }^{-1}$ ) over 14 days at $25^{\circ} \mathrm{C}$ (white), $29^{\circ} \mathrm{C}$ (grey) and $33^{\circ} \mathrm{C}$ (black). Symbiodinium $\mathrm{B} 1, \mathrm{C} 1$ and $\mathrm{E}$ died after seven days at $33^{\circ} \mathrm{C}$. Values are means \pm SE. Symbiodinium B1 $(\mathrm{N}=5-6), \mathrm{C} 1(\mathrm{~N}=3-4), \mathrm{E}(\mathrm{N}=4), \mathrm{F} 1(\mathrm{~N}=3-4)$. Note different scaling. 

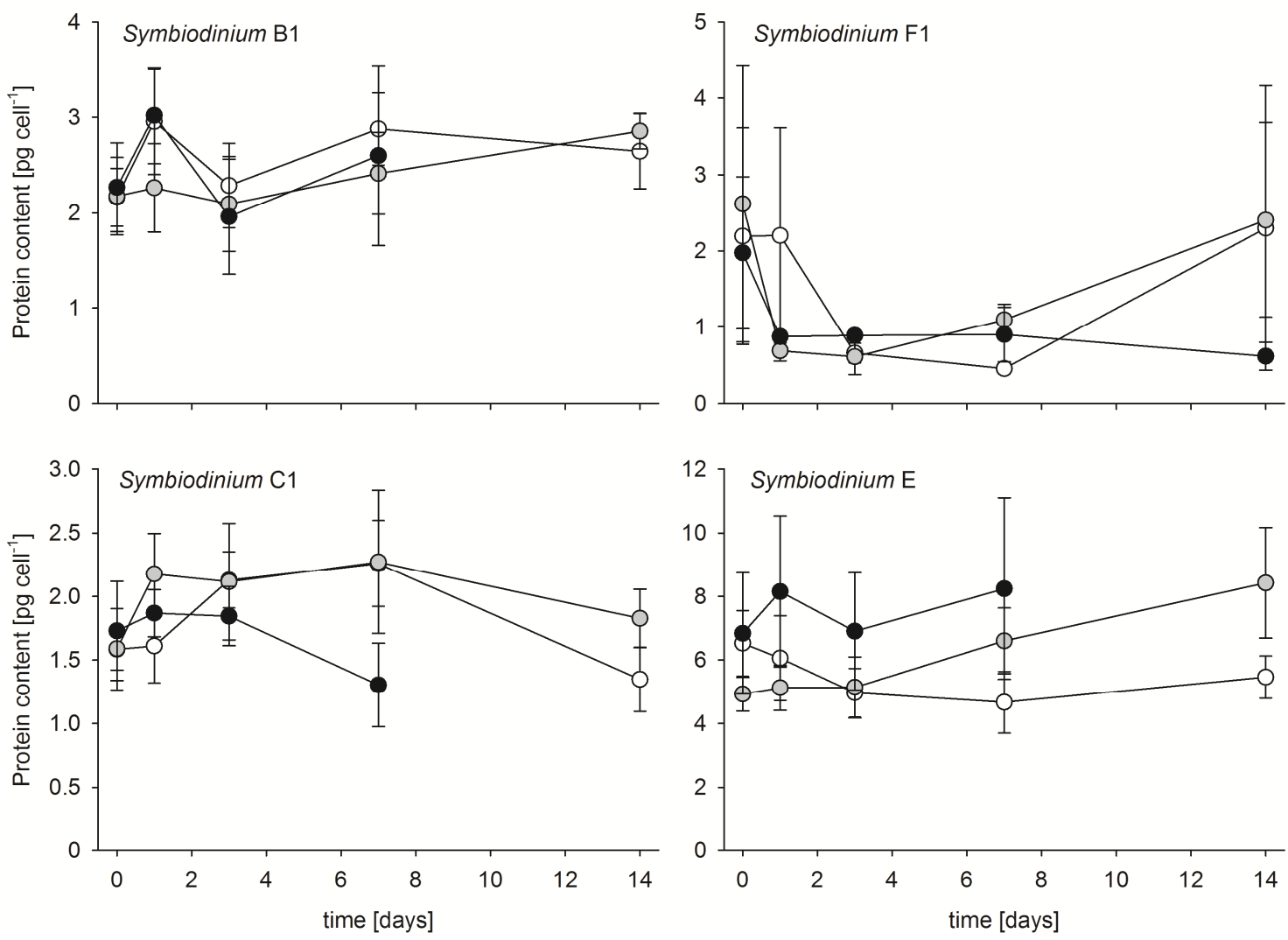

Figure 7.2. Protein content per cell.

Protein content (pg cell ${ }^{-1}$ ) over 14 days at $25^{\circ} \mathrm{C}$ (white), $29^{\circ} \mathrm{C}$ (grey) and $33^{\circ} \mathrm{C}$ (black). Symbiodinium $\mathrm{B} 1, \mathrm{C} 1$ and $\mathrm{E}$ died after seven days at $33^{\circ} \mathrm{C}$. Values are means $\pm \mathrm{SE}$. Symbiodinium B1 $(\mathrm{N}=5-6), \mathrm{C} 1(\mathrm{~N}=3-4), \mathrm{E}(\mathrm{N}=4), \mathrm{F} 1(\mathrm{~N}=3-4)$. Note different scaling. 

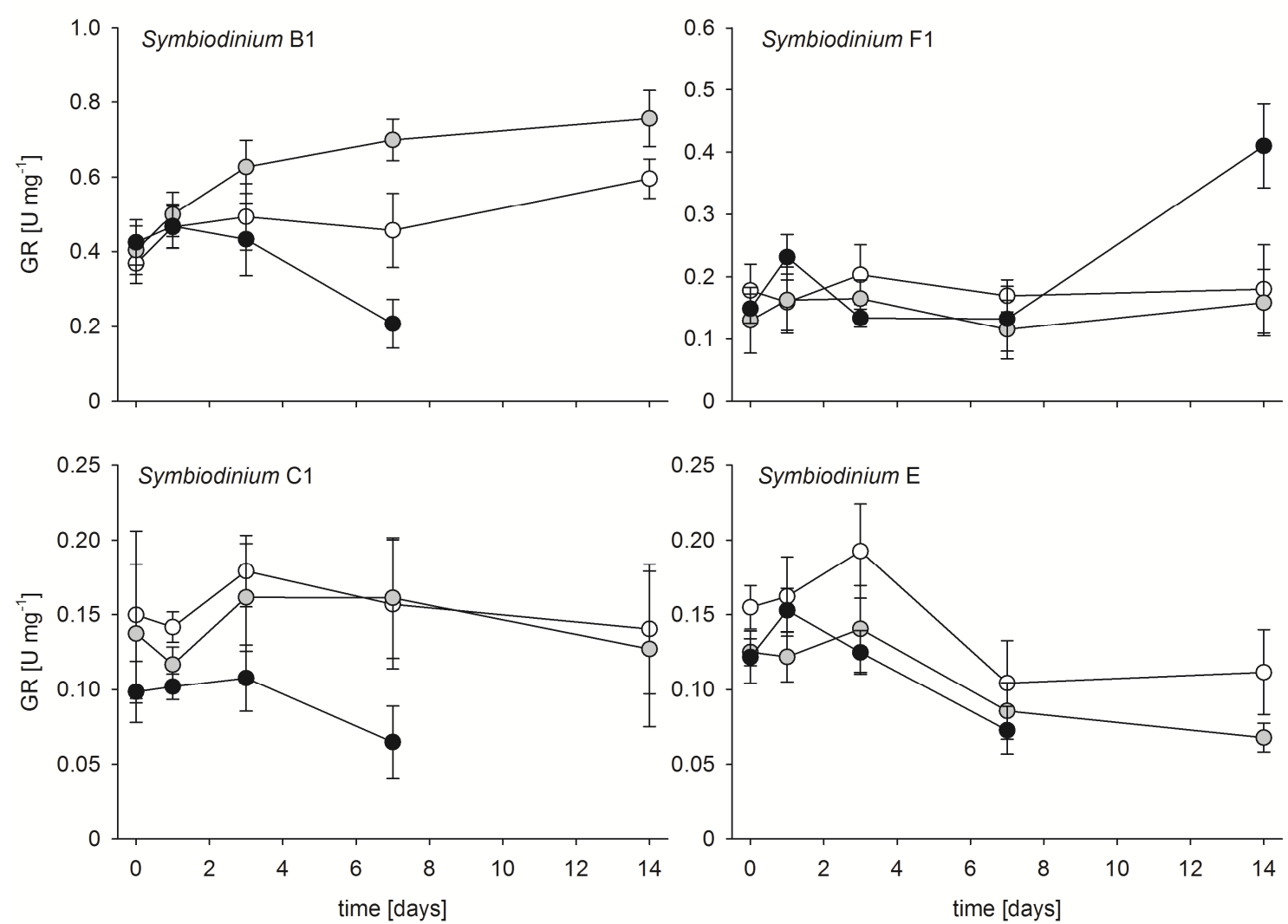

Figure 7.3. Glutathione reductase activity.

Glutathione reductase (GR) activity in units per mg protein over 14 days at $25^{\circ} \mathrm{C}$ (white), $29^{\circ} \mathrm{C}$ (grey) and $33^{\circ} \mathrm{C}$ (black). Symbiodinium B1, C1 and $\mathrm{E}$ died after seven days at $33^{\circ} \mathrm{C}$. Values are means $\pm \mathrm{SE}$. Symbiodinium B1 $(\mathrm{N}=5-6), \mathrm{C} 1(\mathrm{~N}=3-4), \mathrm{E}(\mathrm{N}=4), \mathrm{F} 1$ $(\mathrm{N}=4-5)$. Note different scaling. 

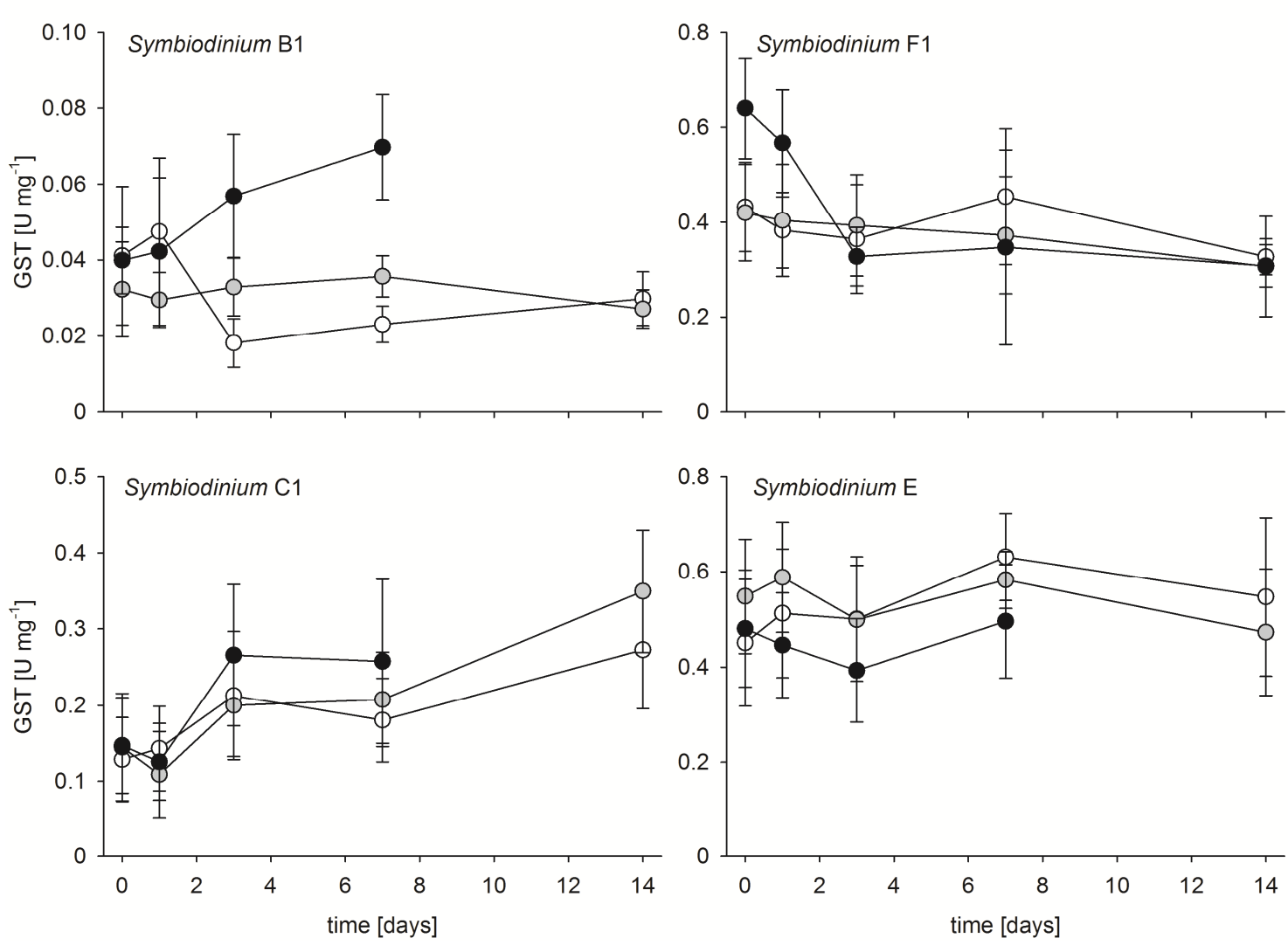

Figure 7.4. Glutathione S-transferase activity.

Glutathione S-transferase (GST) activity in units per mg protein over 14 days at $25^{\circ} \mathrm{C}$ (white), $29^{\circ} \mathrm{C}$ (grey) and $33^{\circ} \mathrm{C}$ (black). Symbiodinium B1, C1 and $\mathrm{E}$ died after seven days at $33^{\circ} \mathrm{C}$. Values are means $\pm S E$. Symbiodinium B1 $(N=5-6), C 1(N=3-4), E(N=4), F 1$ $(\mathrm{N}=3-4)$. Note different scaling. 
Appendix for Chapter 3
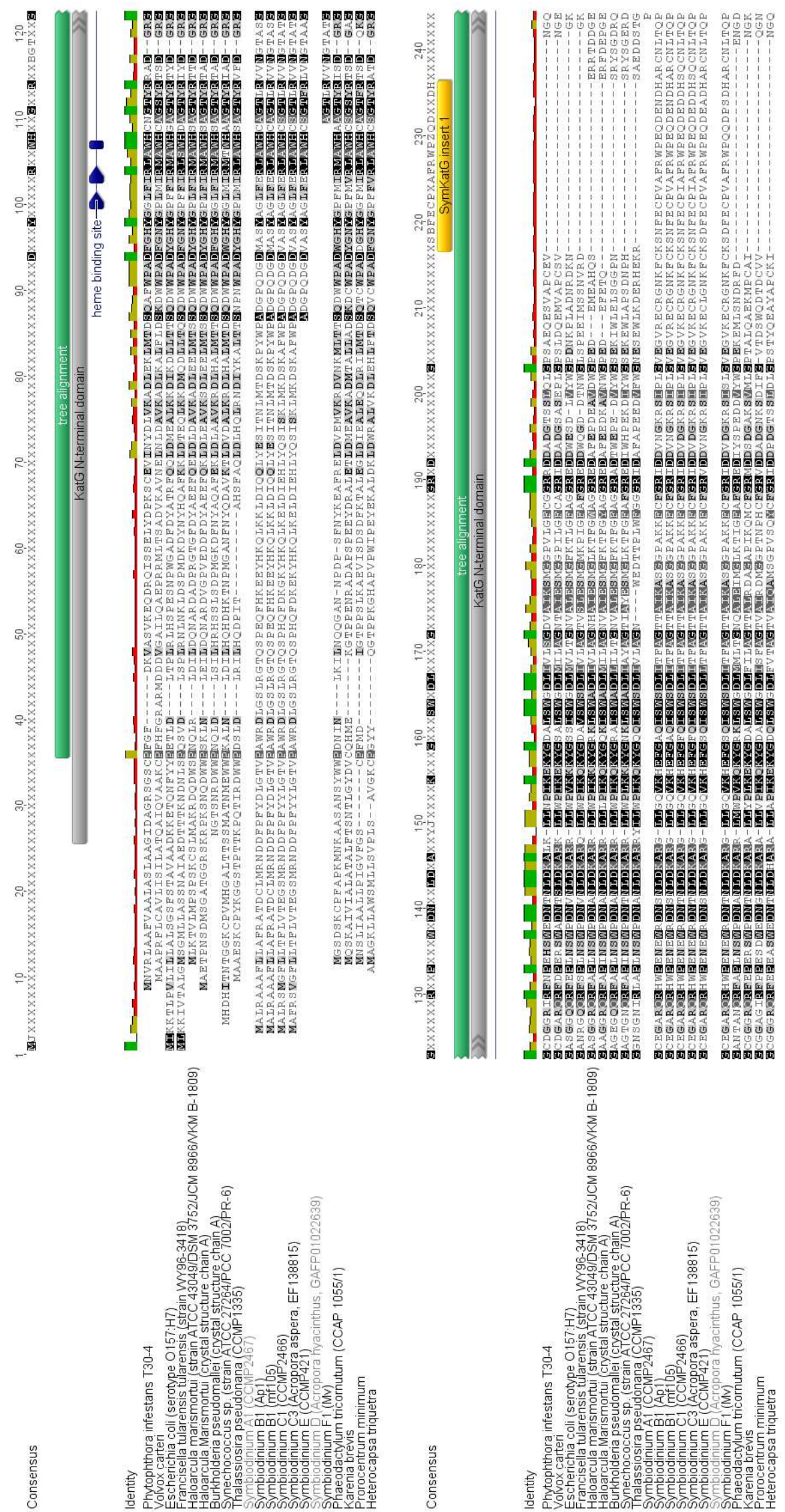
Figure 7.5 [continued]
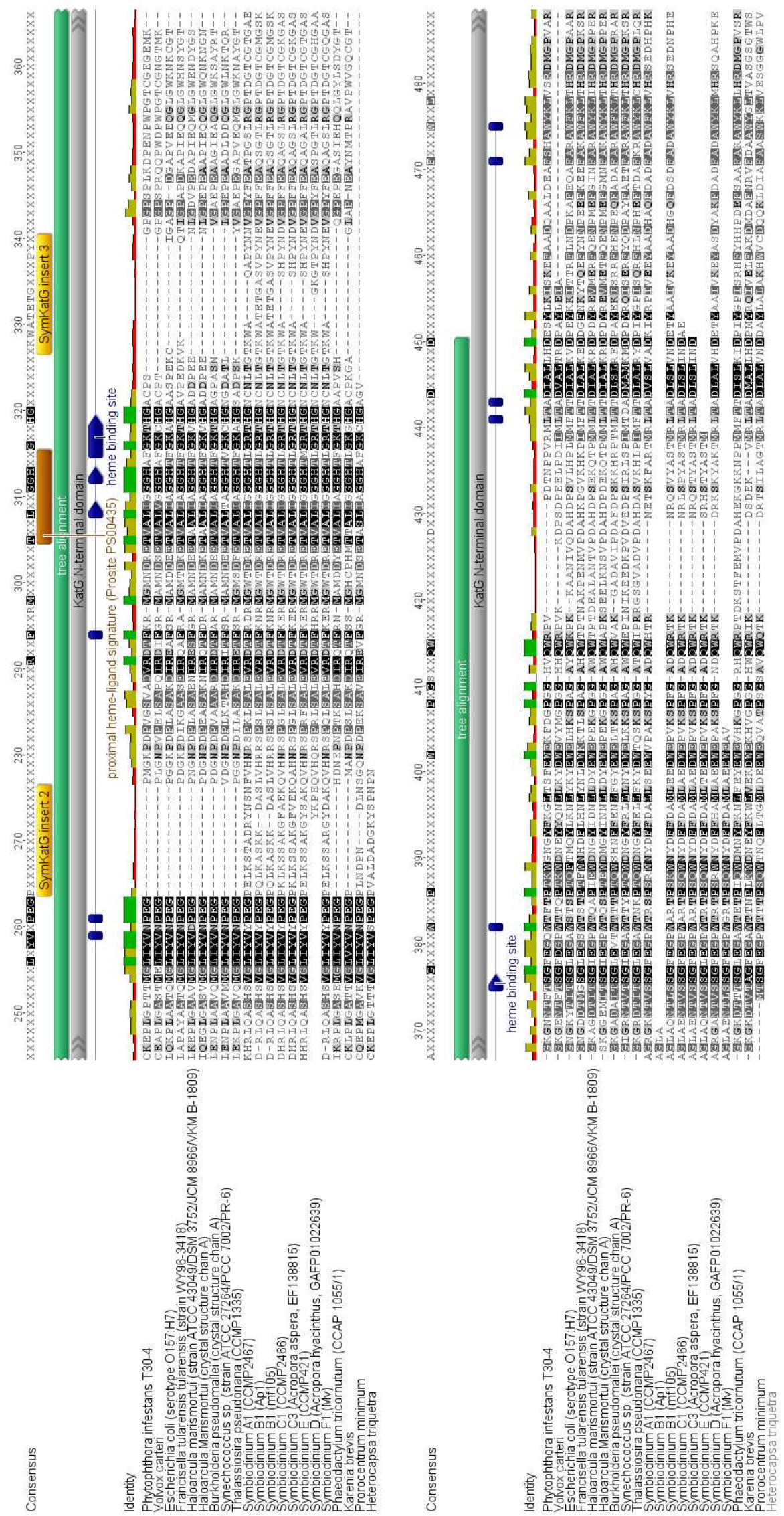
Figure 7.5 [continued]

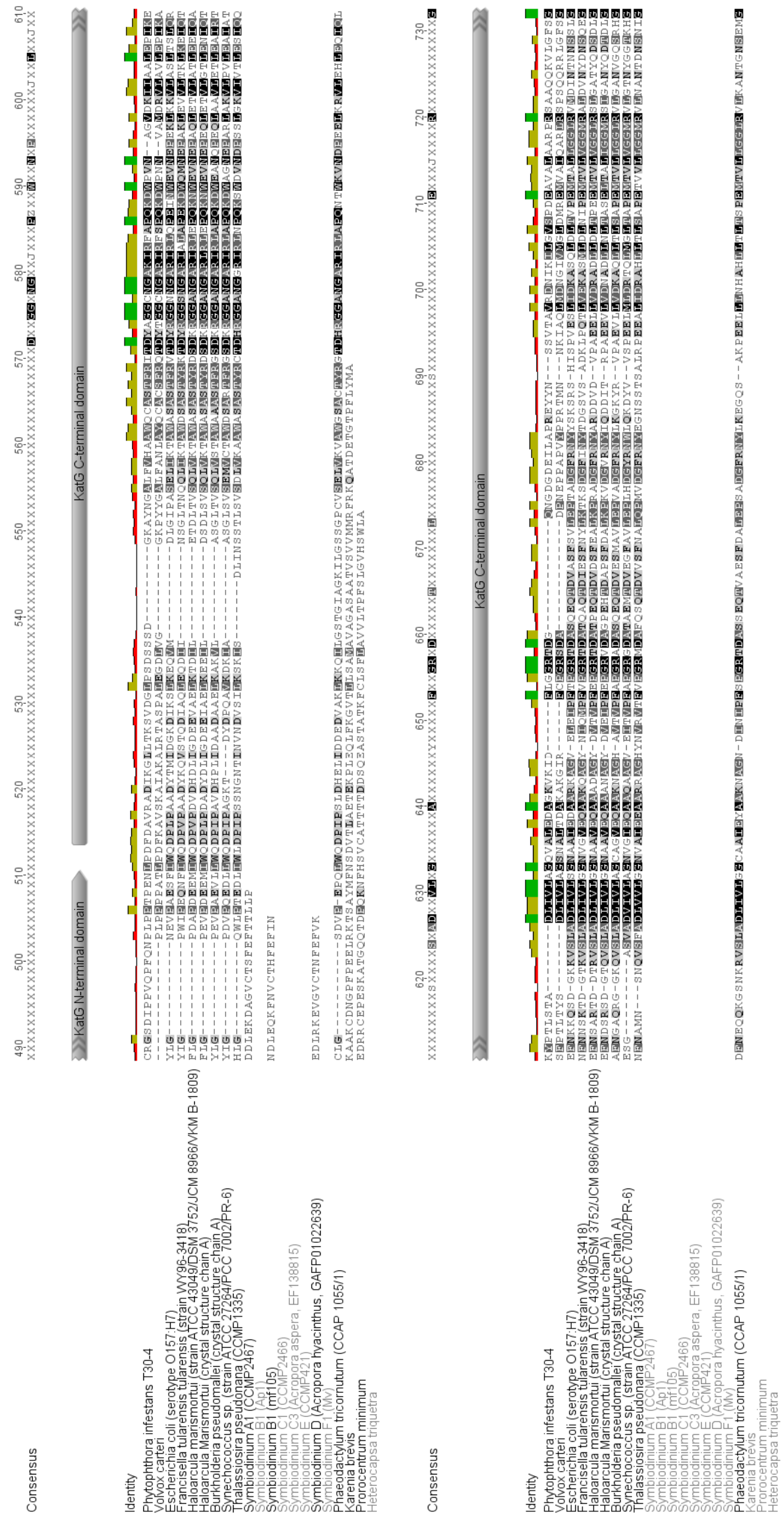


Figure 7.5 [continued]

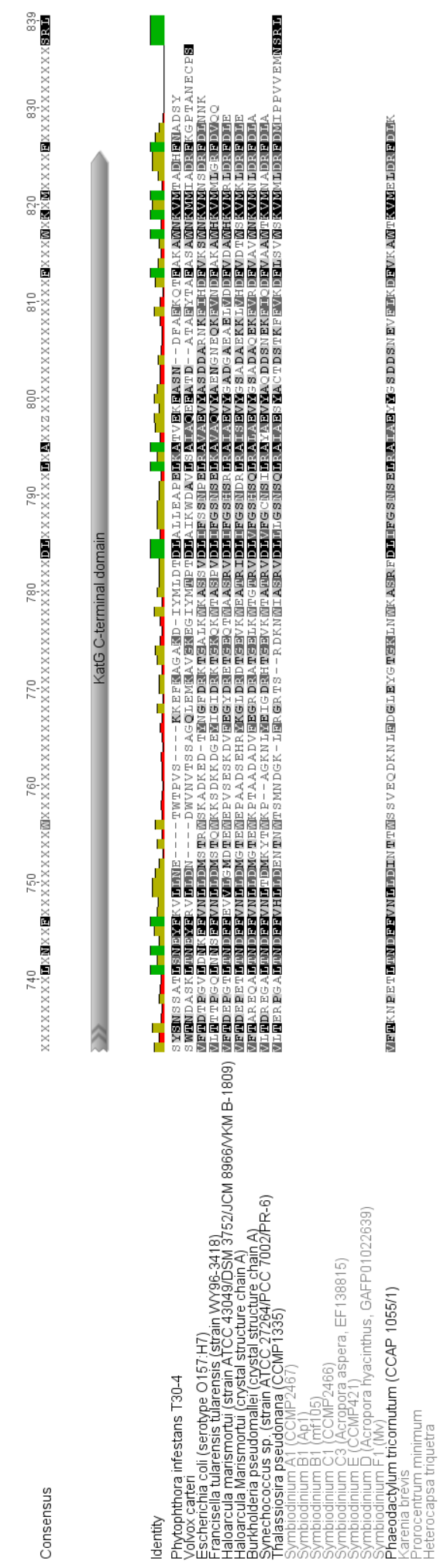

Figure 7.5. Complete KatG alignment.

Complete KatG alignment including $\mathrm{N}$ - and $\mathrm{C}$-terminal domains used for phylogenetic analysis (Figure 3.9) and overall characterization as outlined in Chapter 3. Key residues are highlighted according to Figure 3.7. Length of trimmed alignment used for phylogenetic tree highlighted on consensus (green). 
Table 7.1 Distance matrices for pairwise comparison of amino acid sequences for different Symbiodinium ITS2 types.

Percentage identity (bottom half) and absolute number of amino acid substitutions (top half) for mature proteins of (A) MnSOD, (B) FeSOD, (C) APX and (D) KatG. Colour scale from grey to black indicates increasing similarity and lower number of residue substitutions. Culture ID, host and accession number given in brackets, if applicable. Sequences for B1 (mf105_rep_XXX) and A1 (CCMP2467, Locus XXX) refer to EST entries derived from mf105 library, Medina Lab, Pennsylvania State University, USA and CCMP2467 library, Voolstra Lab, King Abdullah University of Science and Technology, Saudi Arabia.

(A) Manganese superoxide dismutase (MnSOD)

\begin{tabular}{|c|c|c|c|c|c|c|c|c|c|c|}
\hline & $\begin{array}{c}\text { A1 } \\
\text { (CCMP } \\
2467, \\
\text { Locus } \\
7642 \text { ) }\end{array}$ & $\begin{array}{c}\text { A1 } \\
\text { (CCMP } \\
2467, \\
\text { Locus } \\
\text { 9072) }\end{array}$ & $\begin{array}{c}\text { B1 } \\
\text { (mf105_rep_ } \\
\text { c13368) }\end{array}$ & $\begin{array}{c}\text { B1 } \\
\text { (mf105_rep_ } \\
\text { c9010) }\end{array}$ & $\begin{array}{c}\text { B1 } \\
(\mathrm{Ap} 1)\end{array}$ & $\begin{array}{c}\text { C1 } \\
\text { (CCMP } \\
2466)\end{array}$ & $\begin{array}{c}\text { C3 } \\
\text { (Mp) }\end{array}$ & $\begin{array}{c}\mathrm{C} 15 \\
\text { (Montipora } \\
\text { digitata) }\end{array}$ & $\begin{array}{c}\mathrm{F} 1 \\
(\mathrm{CCMP} \\
2468)\end{array}$ & $\begin{array}{c}\text { D } \\
\text { (Acropora } \\
\text { hyacinthus, } \\
\text { GAFP01017 } \\
905)\end{array}$ \\
\hline $\begin{array}{l}\text { A1 (CCMP2467, Locus } \\
\text { 7642) }\end{array}$ & & 1 & 12 & 12 & 15 & 15 & 13 & 13 & 18 & 12 \\
\hline $\begin{array}{l}\text { A1 (CCMP2467, Locus } \\
\text { 9072) }\end{array}$ & $80.0 \%$ & & 10 & 10 & 11 & 9 & 11 & 10 & 9 & 10 \\
\hline $\begin{array}{l}\text { B1 } \\
\text { (mf105_rep_c13368) }\end{array}$ & $92.4 \%$ & $87.7 \%$ & & 6 & 12 & 10 & 10 & 9 & 28 & 17 \\
\hline B1 (mf105_rep_c9010) & $92.4 \%$ & $87.7 \%$ & $97.8 \%$ & & 12 & 10 & 10 & 9 & 25 & 13 \\
\hline B1 (Ap1) & $90.5 \%$ & $86.4 \%$ & $94.9 \%$ & $94.9 \%$ & & 16 & 15 & 15 & 30 & 16 \\
\hline C1 (CCMP2466) & $90.5 \%$ & $88.9 \%$ & $95.7 \%$ & $95.7 \%$ & $93.2 \%$ & & 6 & 5 & 21 & 10 \\
\hline C3 (Mp) & $91.8 \%$ & $86.4 \%$ & $95.7 \%$ & $95.7 \%$ & $93.6 \%$ & $97.4 \%$ & & 5 & 21 & 10 \\
\hline $\begin{array}{l}\text { C15 (Montipora } \\
\text { digitata) }\end{array}$ & $91.8 \%$ & $87.7 \%$ & $96.2 \%$ & $96.2 \%$ & $93.6 \%$ & $97.9 \%$ & $97.9 \%$ & & 16 & 7 \\
\hline F1 (CCMP2468) & $88.6 \%$ & $88.9 \%$ & $89.7 \%$ & $90.7 \%$ & $87.2 \%$ & $91.0 \%$ & $91.0 \%$ & $93.2 \%$ & & 20 \\
\hline $\begin{array}{l}\text { D (Acropora } \\
\text { hyacinthus, } \\
\text { GAFP01017905) }\end{array}$ & $92.4 \%$ & $87.7 \%$ & $93.8 \%$ & $95.2 \%$ & $93.2 \%$ & $95.7 \%$ & $95.7 \%$ & $97.0 \%$ & $92.5 \%$ & \\
\hline
\end{tabular}




\section{(B) Iron superoxide dismutase (FeSOD)}

\begin{tabular}{|l|c|c|c|c|}
\hline & A (PF-2005) & B1 (Ap1) & E (CCMP421) & F1 (Mv) \\
\hline A (PF-2005) & & 8 & 9 & 7 \\
\hline B1 (Ap1) & $94.6 \%$ & & 6 & 6 \\
\hline E (CCMP421) & $94.0 \%$ & $95.5 \%$ & & 0 \\
\hline F1 (Mv) & $94.7 \%$ & $95.5 \%$ & $100.0 \%$ & \\
\hline
\end{tabular}

(C) Ascorbate peroxidase (APX)

\begin{tabular}{|c|c|c|c|c|c|c|c|c|c|}
\hline & $\begin{array}{c}\text { A1 } \\
\text { (CCMP } \\
2467)\end{array}$ & $\begin{array}{l}\text { A1 } \\
\text { (Locus } \\
9887 \text { ) }\end{array}$ & $\begin{array}{c}\text { B1 } \\
\text { (Ap1) }\end{array}$ & $\begin{array}{c}\text { B1 } \\
\text { (mf105_rep } \\
\text { c1624) }\end{array}$ & $\begin{array}{c}\text { C1 } \\
\text { (CCMP } \\
2466)\end{array}$ & $\begin{array}{c}\text { C3 } \\
\text { (Acropora } \\
\text { aspera, } \\
\text { FE865713) }\end{array}$ & $\begin{array}{c}\text { C3 } \\
\text { (Seriatopora } \\
\text { hystrix, } \\
\text { HM156698) }\end{array}$ & $\begin{array}{c}\text { C15 } \\
\text { (Montipora } \\
\text { digitata) }\end{array}$ & $\begin{array}{c}\mathrm{F} 1 \\
\text { (Mv) }\end{array}$ \\
\hline A1 (CCMP2467) & & 84 & 89 & 89 & 85 & $\mathrm{~N} / \mathrm{A}$ & 85 & 96 & 102 \\
\hline A1 (Locus 9887) & $61.8 \%$ & & 91 & 120 & 64 & 48 & 113 & 76 & 133 \\
\hline B1 (Ap1) & $59.5 \%$ & $70.1 \%$ & & 0 & 36 & 12 & 49 & 47 & 85 \\
\hline $\begin{array}{l}\text { B1 } \\
\text { (mf105_rep_c1624) }\end{array}$ & $59.5 \%$ & $66.3 \%$ & $100.0 \%$ & & 36 & 27 & 69 & 47 & 105 \\
\hline C1 (CCMP2466) & $61.4 \%$ & $75.2 \%$ & $86.0 \%$ & $86.0 \%$ & & 0 & 1 & 22 & 65 \\
\hline $\begin{array}{l}\text { C3 } \\
\text { (Acropora aspera, } \\
\text { FE865713) }\end{array}$ & $\mathrm{N} / \mathrm{A}$ & $54.7 \%$ & $80.3 \%$ & $74.5 \%$ & $100.0 \%$ & & 1 & 0 & 37 \\
\hline $\begin{array}{l}\text { C3 } \\
\text { (Seriatopora } \\
\text { hystrix, HM156698) }\end{array}$ & $61.4 \%$ & $68.3 \%$ & $83.9 \%$ & $81.6 \%$ & $99.6 \%$ & $99.2 \%$ & & 23 & 106 \\
\hline $\begin{array}{l}\text { C15 } \\
\text { (Montipora digitata) }\end{array}$ & $56.4 \%$ & $70.3 \%$ & $81.6 \%$ & $81.6 \%$ & $91.4 \%$ & $100.0 \%$ & $91.0 \%$ & & 63 \\
\hline $\mathrm{F} 1(M v)$ & $53.6 \%$ & $62.6 \%$ & $72.0 \%$ & $71.1 \%$ & $74.8 \%$ & $70.6 \%$ & $72.3 \%$ & $75.4 \%$ & \\
\hline
\end{tabular}




\section{(D) Catalase peroxidase (KatG)}

\begin{tabular}{|c|c|c|c|c|c|c|c|c|}
\hline & $\begin{array}{c}\text { A1 } \\
\text { (CCMP } \\
2467)\end{array}$ & $\begin{array}{c}\text { B1 } \\
\text { (Ap1) }\end{array}$ & $\begin{array}{c}\mathrm{B} 1 \\
(\mathrm{mf105})\end{array}$ & $\begin{array}{c}\text { C1 } \\
\text { (CCMP } \\
2466)\end{array}$ & $\begin{array}{c}\text { C3 } \\
\text { (Acropora } \\
\text { aspera, } \\
\text { EF138815) }\end{array}$ & $\begin{array}{c}\mathrm{D} \\
\text { (Acropora } \\
\text { hyacinthus, } \\
\text { GAFP01022 } \\
639)\end{array}$ & $\begin{array}{c}\mathrm{E} \\
(\mathrm{CCMP} 421)\end{array}$ & $\begin{array}{c}\mathrm{F} 1 \\
(\mathrm{Mv})\end{array}$ \\
\hline A1 (CCMP2467) & & 34 & 73 & 45 & 44 & 58 & 40 & 31 \\
\hline B1 (Ap1) & $73.2 \%$ & & 0 & 51 & 51 & 25 & 38 & 33 \\
\hline $\mathrm{B} 1$ (mf105) & $70.9 \%$ & $100.0 \%$ & & 60 & 57 & 59 & 47 & 39 \\
\hline C1 (CCMP2466) & $76.6 \%$ & $85.4 \%$ & $85.7 \%$ & & 5 & 37 & 33 & 24 \\
\hline $\begin{array}{l}\text { C3 (Acropora aspera, } \\
\text { EF138815) }\end{array}$ & $76.8 \%$ & $85.4 \%$ & $86.3 \%$ & $98.8 \%$ & & 36 & 33 & 25 \\
\hline $\begin{array}{l}\text { D (Acropora hyacinthus, } \\
\text { GAFP01022639) }\end{array}$ & $73.5 \%$ & $74.5 \%$ & $73.4 \%$ & $77.6 \%$ & $77.9 \%$ & & 33 & 27 \\
\hline E (CCMP421) & $77.7 \%$ & $86.6 \%$ & $86.2 \%$ & $90.1 \%$ & $90.1 \%$ & $78.3 \%$ & & 29 \\
\hline $\mathrm{F} 1(\mathrm{Mv})$ & $80.3 \%$ & $87.3 \%$ & $86.7 \%$ & $91.7 \%$ & $91.4 \%$ & $79.2 \%$ & $90.0 \%$ & \\
\hline
\end{tabular}



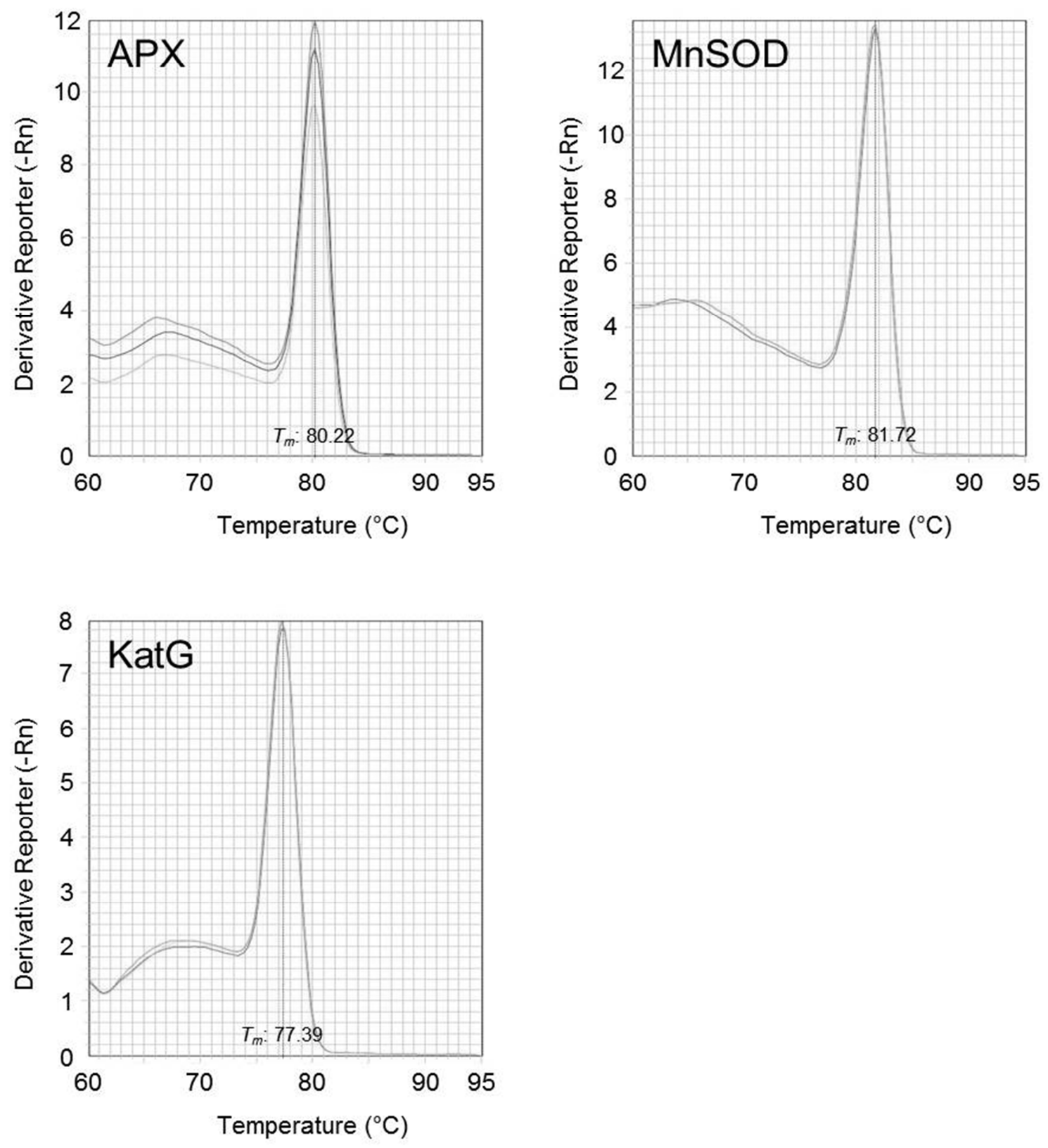

Figure 7.6. Melting curve analysis for genes of interest.

The first derivative of the dissociation of the PCR-product with increasing temperature as measured by the reporter fluorophore (SYBR green) for three technical replicates. Melting temperatures $\left(T_{m}\right)$ of PCR-products for the measured genes of interest, ascorbate peroxidase (APX), manganese superoxide dismutase (MnSOD) and catalase peroxidase (KatG) of Symbiodinium B1, using the qPCR primers listed in Table 3.1, are indicated (dashed line). 

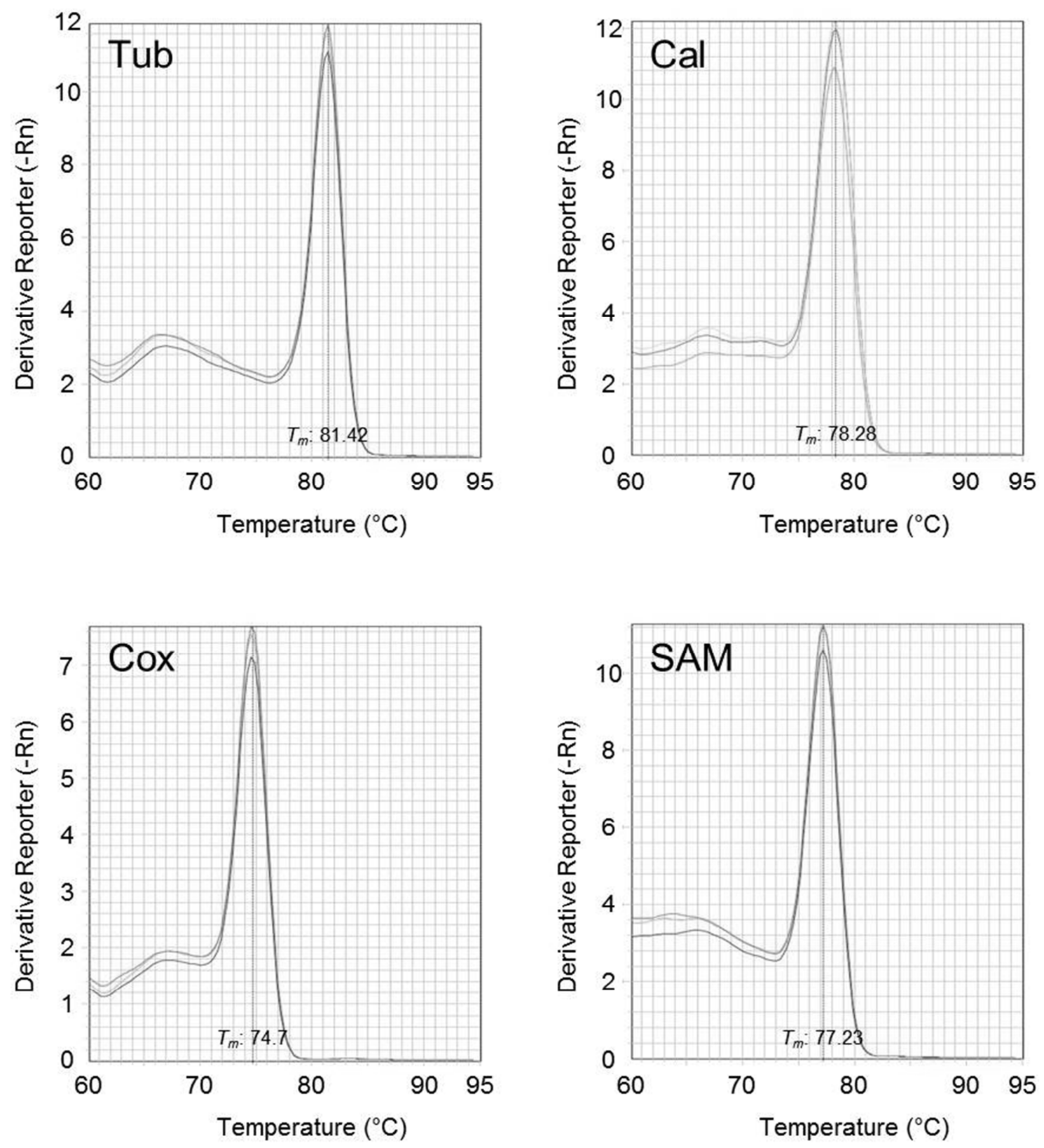

Figure 7.7. Melting curve analysis for housekeeping genes.

The first derivative of the dissociation of the PCR-product with increasing temperature as measured by the reporter fluorophore (SYBR green) for three technical replicates. Melting temperatures $\left(T_{m}\right)$ of PCR-products for the housekeeping genes B-tubulin (Tub), calmodulin (Cal), cytochrome oxidase subunit 1 (Cox) and S-adenosyl methionine synthetase (SAM) of Symbiodinium B1, using the qPCR primers listed in Table 3.1, are indicated (dashed line). 

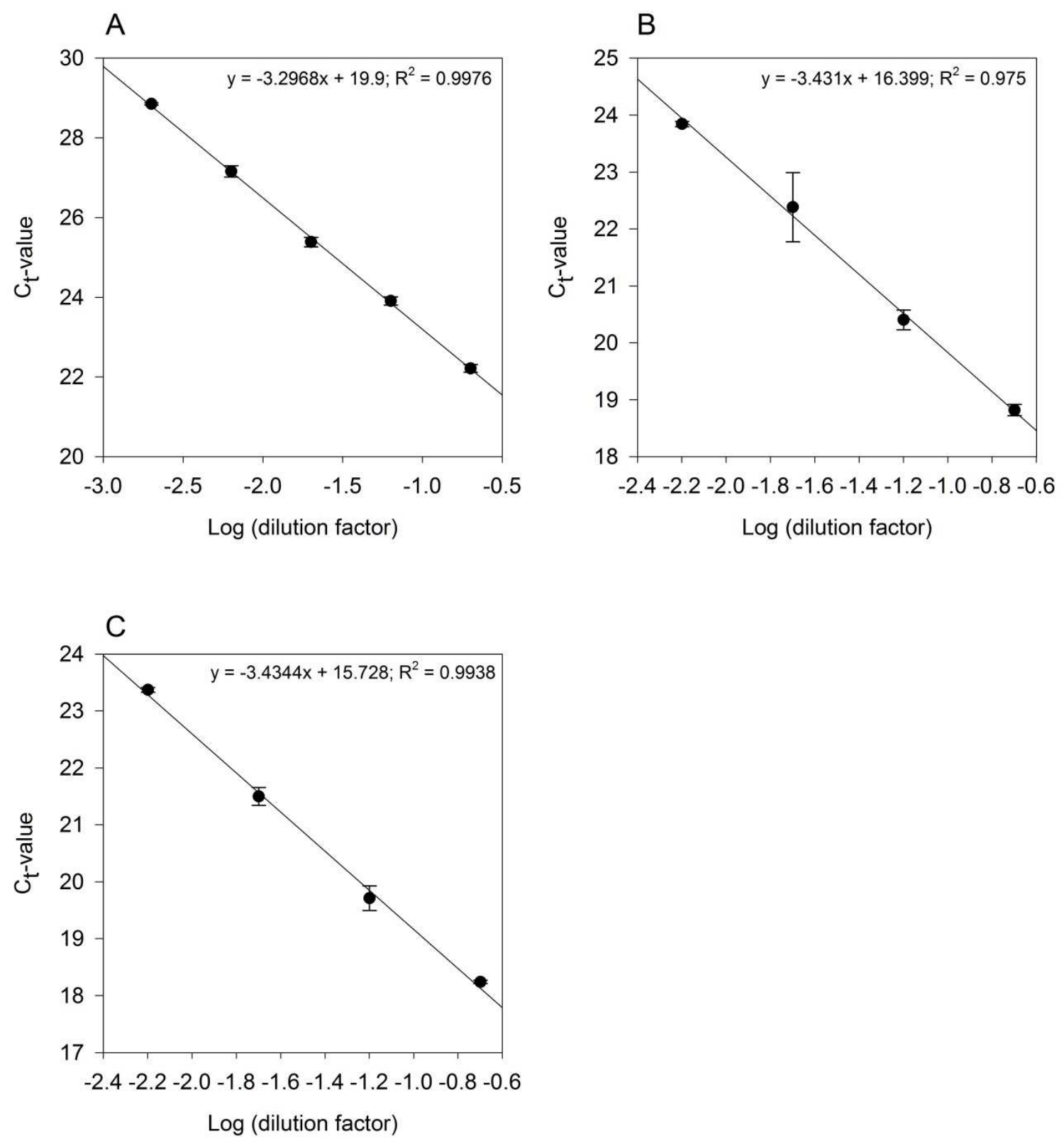

Figure 7.8. Calibration curves for genes of interest in Symbiodinium B1.

Calibration curves used for determination of real-time efficiencies of qPCR primers for genes of interest (cf. Table 3.1). Depicted are $\mathrm{C}_{\mathrm{t}}$-values versus dilution ranges of cDNA samples from Symbiodinium B1 (see 0). Equations of linear regressions used for calculating efficiencies $E$ according to the equation $E=-1+10^{\wedge}[-1 /$ slope $]$, are given for each graph together with their coefficient of determination. (A) ascorbate peroxidase, (B) manganese superoxide dismutase, $(C)$ catalase peroxidase. Values are means \pm SD of three technical replicates. 

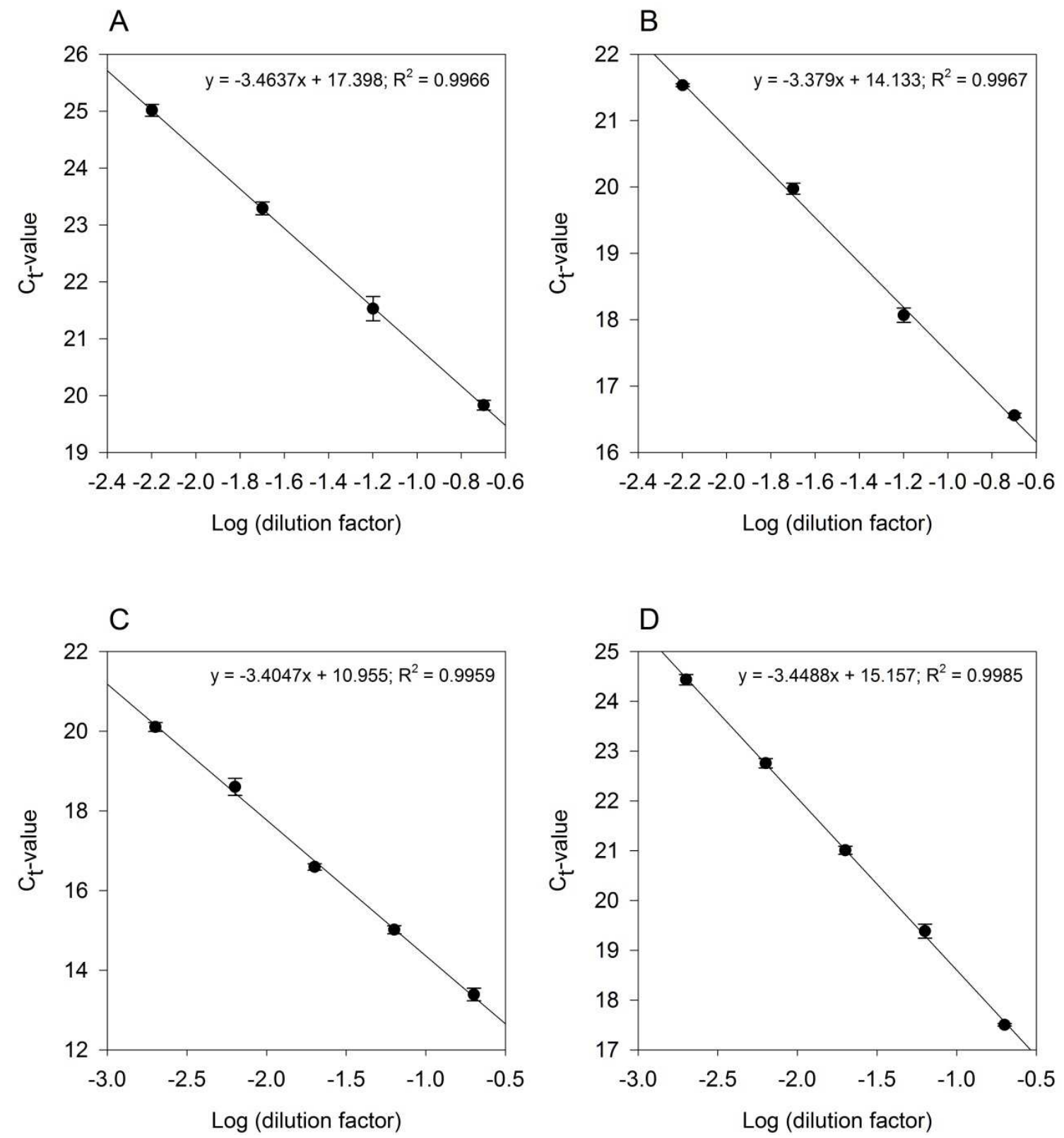

Figure 7.9. Calibration curves for potential housekeeping genes in Symbiodinium B1.

Calibration curves used for determination of real-time efficiencies of qPCR primers for genes of interest (cf. Table 3.1). Depicted are $\mathrm{C}_{\mathrm{t}}$-values versus dilution ranges of cDNA samples from Symbiodinium B1 (see 0). Equations of linear regressions used for calculating efficiencies $E$ according to the equation $E=-1+10^{\wedge}[-1 /$ slope], are given for each graph together with their coefficient of determination. (A) B-tubulin, (B) calmodulin, (C) cytochrome oxidase subunit 1, (D) S-adenosyl methionine synthetase. Values are means \pm SD of three technical replicates. 


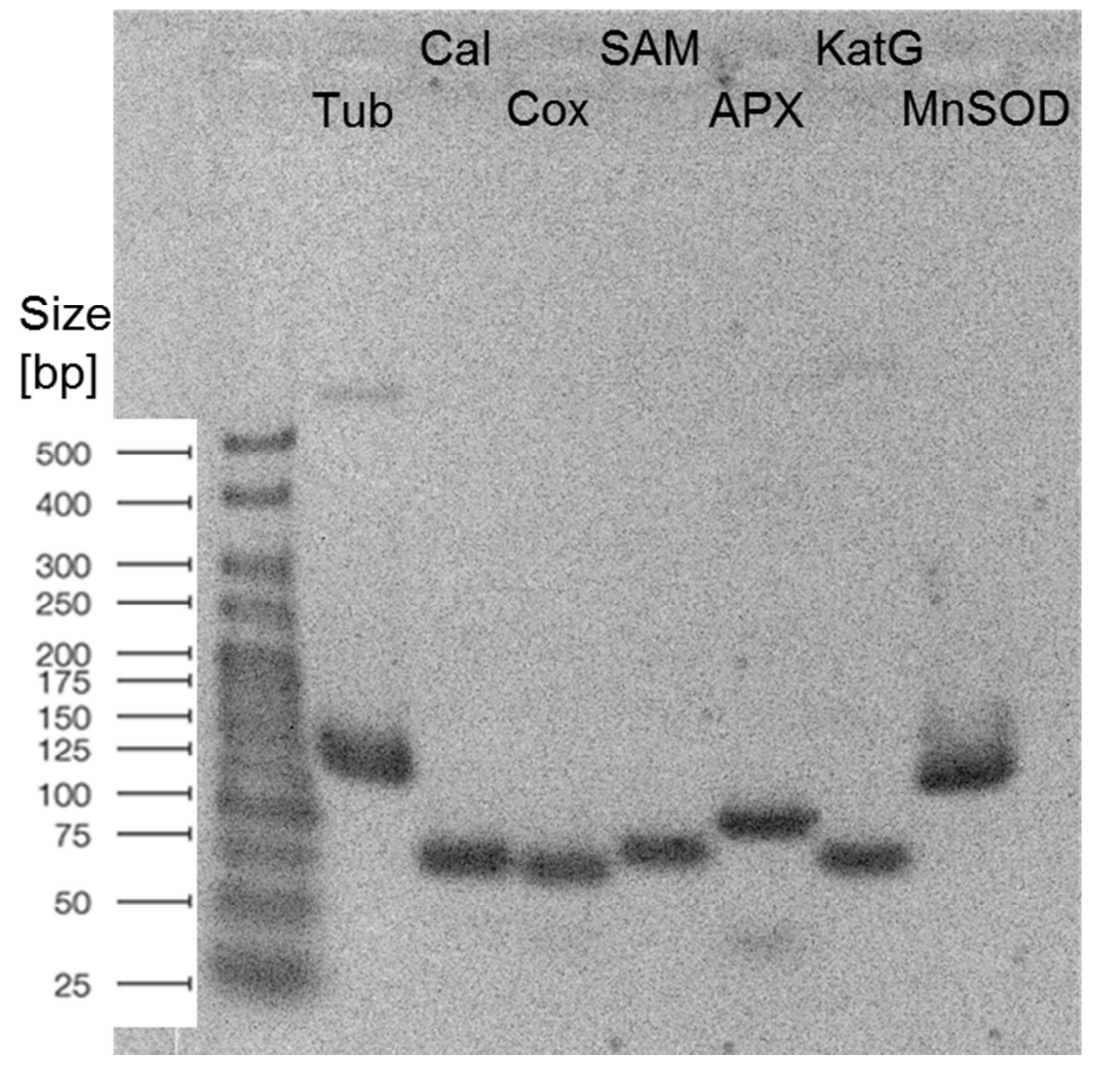

Figure 7.10. Gel electrophoresis of PCR amplicons for suitable qPCR primers in Symbiodinium B1.

PCR amplicon sizes for all potential housekeeping genes (B-tubulin [Tub], calmodulin [Cal], cytochrome oxidase subunit 1 [Cox], S-adenosyl methionine synthetase [SAM]) and genes of interest (ascorbate peroxidase [APX], catalase peroxidase [KatG], manganese superoxide dismutase [MnSOD]) for Symbiodinium B1 (cf. Table 3.1). PCR consisted of 40 cycles with an annealing temperature of $60^{\circ} \mathrm{C}$. PCR products were visualized on $3.5 \%[\mathrm{w} / \mathrm{v}]$ agarose with ethidium bromide, using Hyperladder $\mathrm{V}$ as the size marker. 


\section{Appendix for Chapter 4}
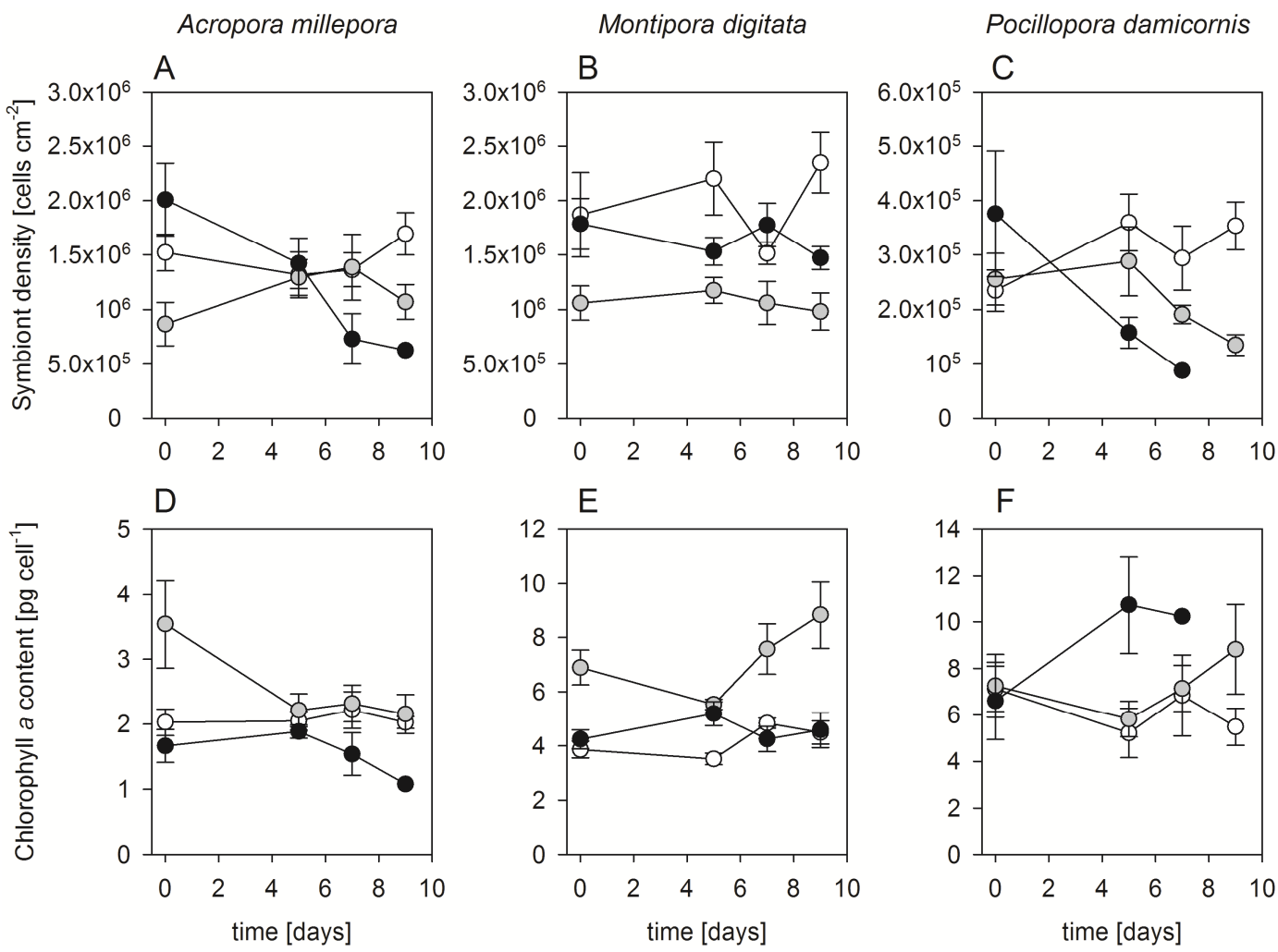

Figure 7.11. Symbiont density and chlorophyll a content.

Symbiont density and chlorophyll a content per cell for Acropora millepora, Montipora digitata and Pocillopora damicornis exposed to $28^{\circ} \mathrm{C}$ (white), $31^{\circ} \mathrm{C}$ (grey) and $33^{\circ} \mathrm{C}$ (black) over nine days. Values are means $\pm \mathrm{SE}, \mathrm{N}=5$, except for Day 9 in $A$. millepora at $33^{\circ} \mathrm{C}(\mathrm{N}=2)$ and for Day 7 in $P$. damicornis at $33^{\circ} \mathrm{C}(\mathrm{N}=1)$. Note different scaling. 


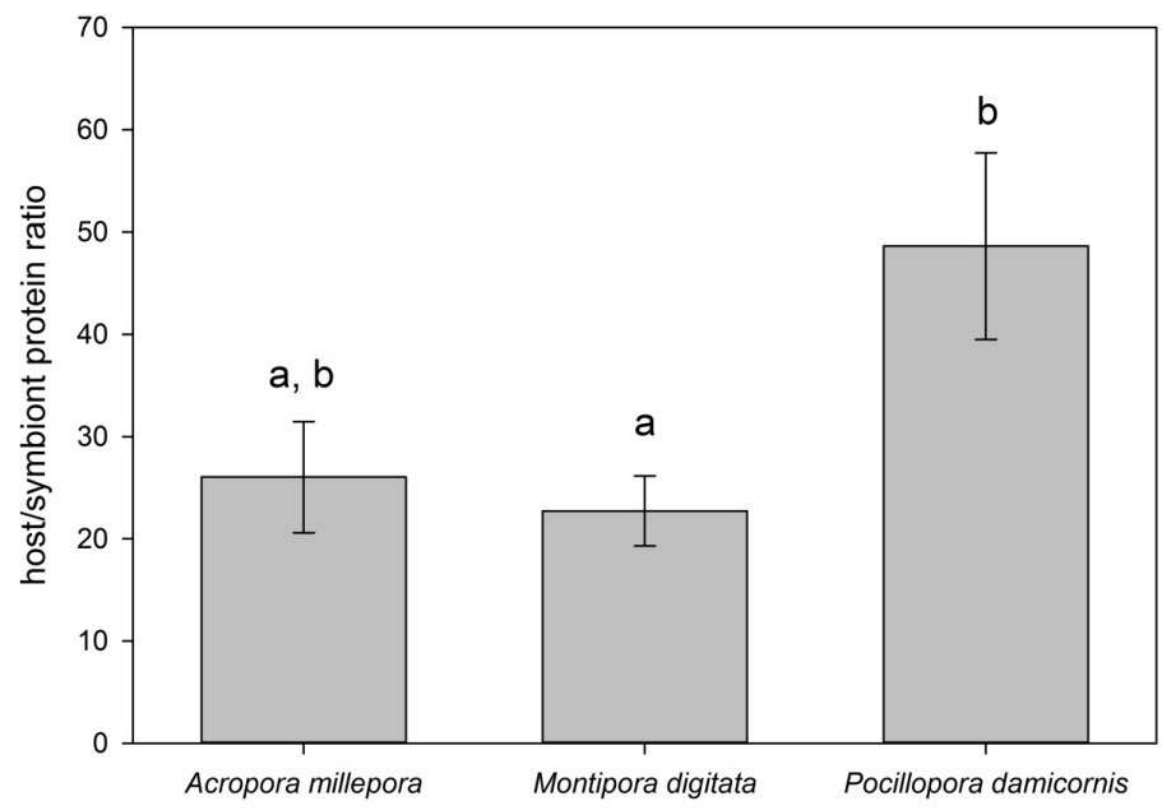

Figure 7.12. Ratios between total host and symbiont protein.

Ratios between total protein content of the host and symbionts, for three coral species on Day 0 at $28^{\circ} \mathrm{C}$. Values are means $\pm \mathrm{SE}(\mathrm{N}=5$, except for $P$. damicornis, where $\mathrm{N}=$ 4 ). Bars that share the same letter are not significantly different from each other (oneway ANOVA, $F_{2,11}=5.1519, p=0.0264$ with post hoc Tukey HSD test).
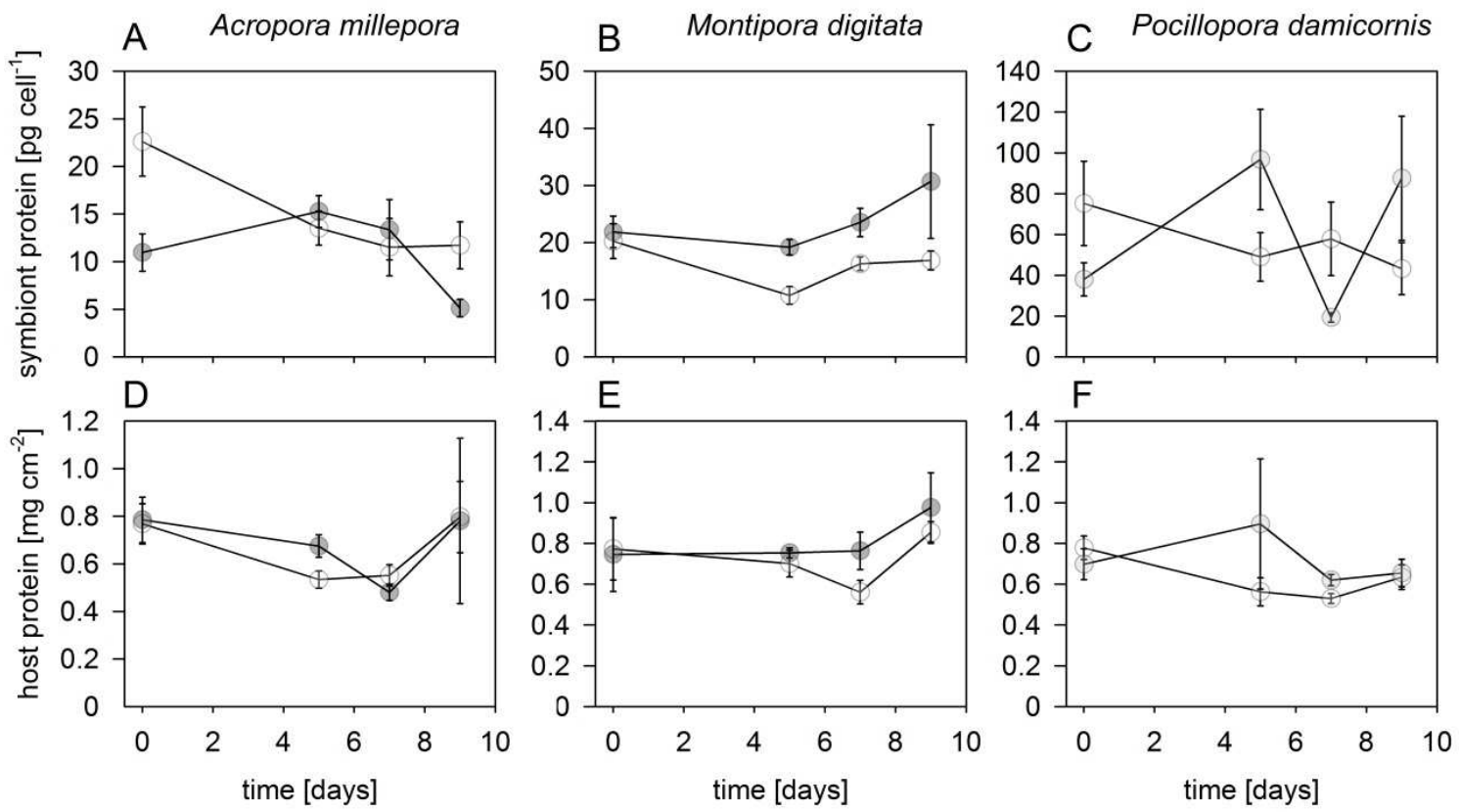

Figure 7.13. Protein contents for host and symbiont.

Shown are the relative protein contents for the symbiont $(A-C)$ and the host (D-F) for $28^{\circ} \mathrm{C}$ (white), $31^{\circ} \mathrm{C}$ (grey) and $33^{\circ} \mathrm{C}$ (dark grey). Values represent measurements from colony explants that were analysed for NO synthesis and host apoptotic-like pathways in the same experiment (Hawkins et al., 2014). Values are means $\pm S E(N=4-5)$. 
Figure 7.14. ITS2 rDNA signatures of isolated coral symbionts.

M.digitata 1

M.digitata 2

M.digitata 3

M.digitata 4

M.digitata 5

P.damicornis 1

P.damicornis 2

P.damicornis 3

P.damicornis 4

P.damicornis 5

A. millepora 1

A. millepora 2

A. millepora 3

A. millepora 4

A. millepora 5

M.digitata 1

M.digitata 2

M.digitata 3

M.digitata 4

M.digitata 5

P.damicornis 1

P.damicornis 2

P.damicornis 3

P.damicornis 4

P.damicornis 5

A. millepora 1

A. millepora 2

A. millepora 3

A. millepora 4

A. millepora 5

$\cdots|\ldots|_{10} \ldots|\cdots|_{20} \ldots|\cdots|_{30}$

30

40

$\ldots|\ldots|_{50} \ldots|\cdots|_{60} \ldots|\ldots|_{70}$

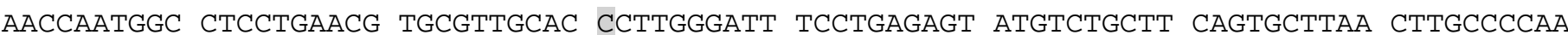
AACCAATGGC CTCCTGAACG TGCGTTGCAC CCTTGGgAtT TCCTGAGAgT ATGTCTGCTT CAGTGCTTAA CTTGCCCCAA AACCAATGGC CTCCTGAACG TGCGTTGCAC CCTTGGgAtT TCCTGAGAGT ATGTCTGCTT CAGTGCTTAA CTTGCCCCAA

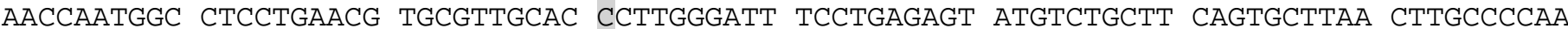
AACCAATGGC CTCCTGAACG TGCGTTGCAC CCTTGGgAtT TCCTGAGAGT ATGTCTGCtT CAGTGCTTAA CTtGCCCCAA

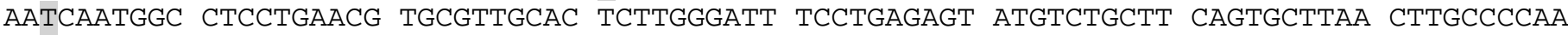
AATCAATGgC CTCCTGAACG TGCGTTGCAC TCTTGgGATT TCCTGAGAgT ATGTCTGCTT CAgTGCTTAA CTTGCCCCAA

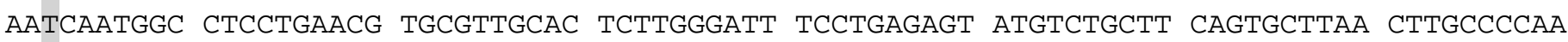

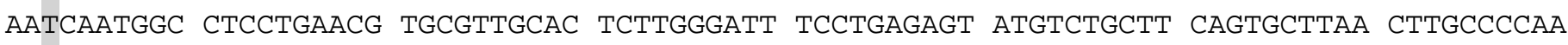

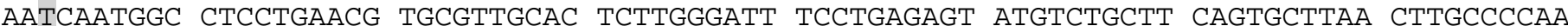

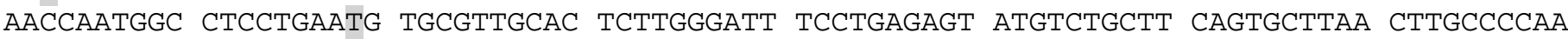
AACCAATGGC CTCCTGAATg TGCGTTGCAC TCTTGgGATt TCCTGAgAgt ATGTCTGCTT CAGTGCTTAA CTTGCCCCAA

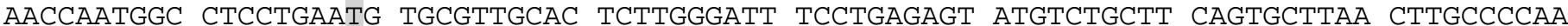
AACCAATGGC CTCCTGAATg TGCGTTGCAC TCTTGGGATT TCCTGAGAGT ATGTCTGCTT CAGTGCTTAA CTTGCCCCAA

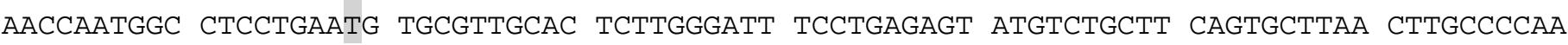
$\ldots|\ldots|_{90} \ldots|\ldots|_{100} \ldots|\ldots|_{110} \ldots|\ldots|_{120} \ldots|\ldots|_{130} \ldots|\ldots|_{140} \ldots|\ldots| \ldots|\ldots| \ldots \mid$

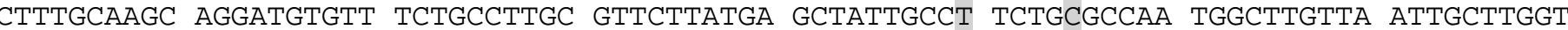
CTTTGCAAGC AGgATGTGTT TCTGCCTTGC GTTCTTATGA GCTATTGCCT TCTGCGCCAA TGGCTTGTTA ATtGCTTGGT

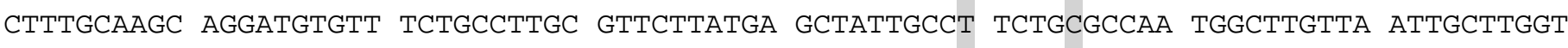

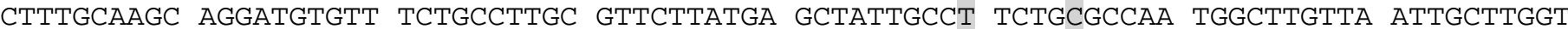
CTTTGCAAGC AgGATGTGTT TCTGCCTTGC GTTCTTATGA GCTATTGCCT TCTGCGCCAA TGGCTTGTTA ATtGCTTGGT

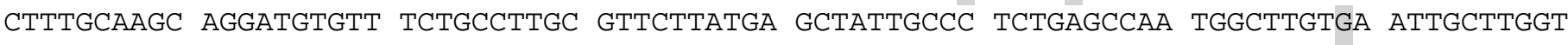

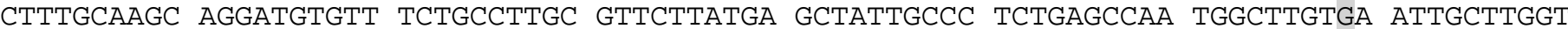
CTTTGCAAGC AgGATGTGTT TCTGCCTTGC GTTCTTATGA GCTATTGCCC TCTGAgCCAA TGgCTTGTGA ATtGCTTGGT CTTTGCAAGC AGGATGTGTT TCTGCCTTGC GTTCTTATGA GCTATTGCCC TCTGAGCCAA TGGCTTGTGA ATTGCTTGGT

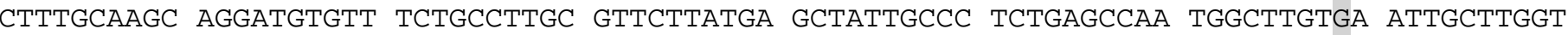
CTTTGCAAGC AgGATGTGTT TCTGCCTTGC GTTCGTATGA GTTATTGCCC TCTGAgCCAA TGGCTTGTTA ATtGCTTGGT CTTTGCAAGC AGgATGTGTT TCTGCCTTGC GTTCGTATGA GTTATTGCCC TCTGAGCCAA TGGCTTGTTA ATtGCTTGGT

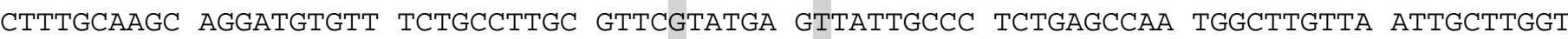

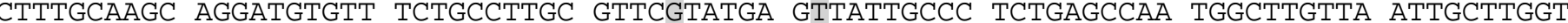

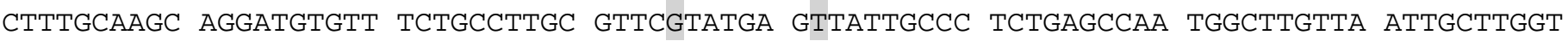


Figure 7.14 [continued]

M.digitata 1

M.digitata 2

M.digitata 3

M.digitata 4

M.digitata 5

P.damicornis 1

P.damicornis 2

P.damicornis 3

P.damicornis 4

P.damicornis 5

A. millepora 1

A. millepora 2

A. millepora 3

A. millepora 4

A. millepora 5

M.digitata 1

M.digitata 2

M.digitata 3

M.digitata 4

M.digitata 5

P.damicornis 1

P.damicornis 2

P.damicornis 3

P.damicornis 4

P.damicornis 5

A. millepora 1

A. millepora 2

A. millepora 3

A. millepora 4

A. millepora 5

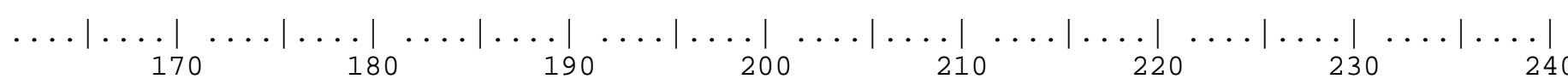

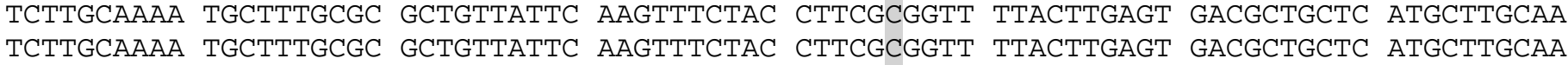
TCTTGCAAAA TGCTTTGCGC GCTGTTATTC AAGTTTCTAC CTTCGCGGTT TTACTTGAGT GACGCTGCTC ATGCTTGCAA TCTTGCAAAA TGCTTTGCGC GCTGTTATTC AAgTTTCTAC CTTCGCGGTT TTACTTGAgT GACGCTGCTC ATGCTTGCAA TCTTGCAAAA TGCTTTGCGC GCTGTTATTC AAGTTTCTAC CTTCGCGGTT TTACTTGAGT GACGCTGCTC ATGCTTGCAA

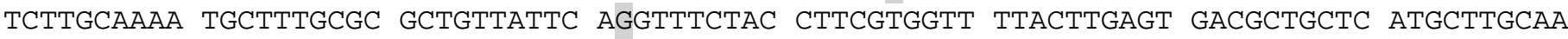
TCTTGCAAAA TGCTTTGCGC GCTGTTATTC AGGTTTCTAC CTTCGTGGTT TTACTTGAGT GACGCTGCTC ATGCTTGCAA TCTTGCAAAA TGCTTTGCGC GCTGTTATTC AGgTTTCTAC CTTCGTGGTT TTACTTGAGT GACGCTGCTC ATGCTTGCAA

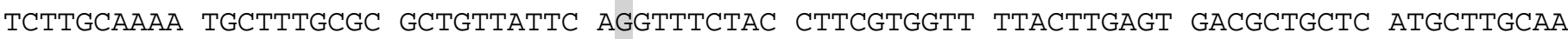
TCTTGCAAAA TGCTTTGCGC GCTGTTATTC AgGTTTCTAC CTTCGTGGTT TTACTTGAgt GACGCTGCTC ATGCTTGCAA TCTTGCAAAA TGCTTTGCGC GCTGTTATTC AAGTTTCTAC CTTCGTGGTT TTACTTGAgt GACGCTGCTC ATGCTTGCAA

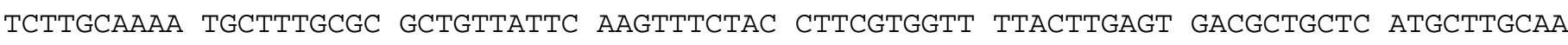
TCTTGCAAAA TGCTTTGCGC GCTGTTATTC AAgTTTCTAC CTTCGTGGTT TTACTTGAgT GACGCTGCTC ATGCTtGCAA TCTTGCAAAA TGCTTTGCGC GCTGTTATTC AAGTTTCTAC CTTCGTGGTT TTACTTGAGT GACGCTGCTC ATGCTTGCAA

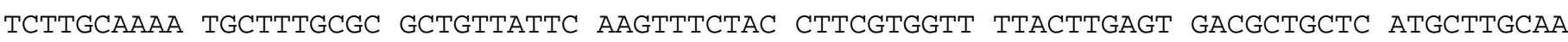

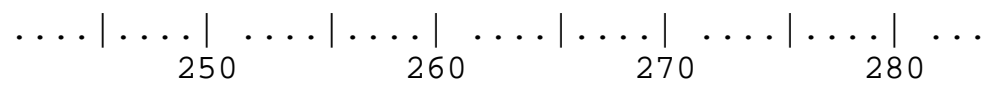

CCGCTGGGAT GCAGGTGCAT GCCTCTAGCA TGAAGTCAGA CAA CCGCTGGGAT GCAGGTGCAT GCCTCTAGCA TGAAGTCAGA CAA CCGCTGGGAT GCAGGTGCAT GCCTCTAGCA TGAAGTCAGA CAA CCGCTGGGAT GCAGGTGCAT GCCTCTAGCA TGAAGTCAGA CAA CCGCTGGGAT GCAGGTGCAT GCCTCTAGCA TGAAGTCAGA CAA CCGCTGGGAT GCAGGTGCAT GCCTCTAGCA TGAAGTCAGA CAA CCGCTGGGAT GCAGGTGCAT GCCTCTAGCA TGAAGTCAGA CAA CCGCTGGgAT GCAGGTGCAT GCCTCTAGCA TGAAgTCAgA CAA CCGCTGGGAT GCAGGTGCAT GCCTCTAGCA TGAAGTCAGA CAA CCGCTGGGAT GCAGGTGCAT GCCTCTAGCA TGAAGTCAGA CAA CCGCTGGGAT GCAGGTGCAT GCCTCTAGCA TGAAGTCAGA CAA CCGCTGGGAT GCAGGTGCAT GCCTCTAGCA TGAAGTCAGA CAA CCGCTGGGAT GCAGGTGCAT GCCTCTAGCA ---------- --CCGCTGGGAT GCAGgTGCAT GCCTCTAGCA TGAAgTCAGA CAA CCGCTGGgAt GCAGGTGCAT GCCTCTAGCA TGAAGTCAGA CAA 


\section{Additional work}

\section{Effects of temperature priming on thermal response at $33^{\circ} \mathrm{C}$ in Symbiodinium B1}

Symbiodinium batch cultures are usually maintained at constant temperatures in the lab, before being used in experimental treatments. This experiment was designed to assess whether the mode of thermal acclimation (constant vs. fluctuating) has an effect on the temperature response of Symbiodinium B1 to $33^{\circ} \mathrm{C}$.

\section{Experimental design}

A batch culture of Symbiodinium B1 (culture ID Ap1, maintained at $25^{\circ} \mathrm{C}$ ) was split into two subcultures and acclimated either at a constant temperature of $24.7^{\circ} \mathrm{C} \pm 0.29^{\circ} \mathrm{C}$ (mean $\pm S D, N=4489$ ), or a diel temperature cycle between $24.5^{\circ} \mathrm{C}$ and $28^{\circ} \mathrm{C}$, with an average of $25.4^{\circ} \mathrm{C} \pm 1.29^{\circ} \mathrm{C}$ (Figure 8.1 ). The subcultures were grown in sterile $\mathrm{f} / 2$-medium (natural seawater base) and acclimated for 32 days under a $12 \mathrm{~h} \mathrm{~L} / \mathrm{D}$ cycle of $65-70 \mu$ mol quanta $\mathrm{m}^{-2} \mathrm{~s}^{-1}$ (cool white fluorescent tubes, Philips 36W/840). At the end of the acclimation period, each subculture was used to start eight culture flasks with a cell density of $\sim 1 \times 10^{5}$ cells $\mathrm{mL}^{-1}$, respectively. Four randomly picked flasks from each acclimation mode were then allocated to a third tank, while the rest was maintained under their respective acclimation treatment. On the next day (Day 0 of the experiment), three $50 \mathrm{~mL}$ culture aliquots from all flasks were harvested, pelleted $(2000 \times \mathrm{g}, 5$ min, $25^{\circ} \mathrm{C}$ ), and immediately frozen at $-80^{\circ} \mathrm{C}$. After sampling on Day 0 , the temperature in the third tank was increased from $25^{\circ} \mathrm{C}$ to $33^{\circ} \mathrm{C}$ by a rate of $1.25^{\circ} \mathrm{C}$ hour $^{-1}$, and maintained at this temperature. All three tanks were sampled on Days 0, 3, and 6 between 13:00 and 15:00. Temperature in each tank was monitored via HOBO Pendant $\AA^{\circledR}$ data loggers (Onset Computer Corporation, Bourne, MA, USA).

To assess the effect of acclimation on the temperature response, maximum quantum yield $\left(\mathrm{F}_{\mathrm{v}} / \mathrm{F}_{\mathrm{m}}\right)$, chlorophyll a content, cell growth, protein content, superoxide dismutase (SOD) activity, and ascorbate peroxidase (APX) activity 
were monitored (according to protocols given in Chapter 2 and Chapter 3). PAM and density data were arcsine square root transformed and log transformed, respectively, for statistical analysis. Normality of data was confirmed with the Shapiro-Wilk test and sphericity assumptions tested using Mauchly's sphericity test. Effects of temperature and acclimation mode on all variables were analyzed using repeated measures ANOVA.

\section{Results}

While $F_{v} / F_{m}$ and cell growth significantly declined at $33^{\circ} \mathrm{C}$, protein content, SOD activity, and APX activity were significantly elevated after six days (Table 8.1, Figure 8.2). Acclimation mode had no effect on any of the physiological variables, except for $F_{v} / F_{m}$. Values declined significantly stronger when cultures were acclimated to a fluctuating temperature (-38\% relative to control), compared to a constant temperature $(-22 \%)$ (post hoc contrast, $\mathrm{p}<0.0001)$.

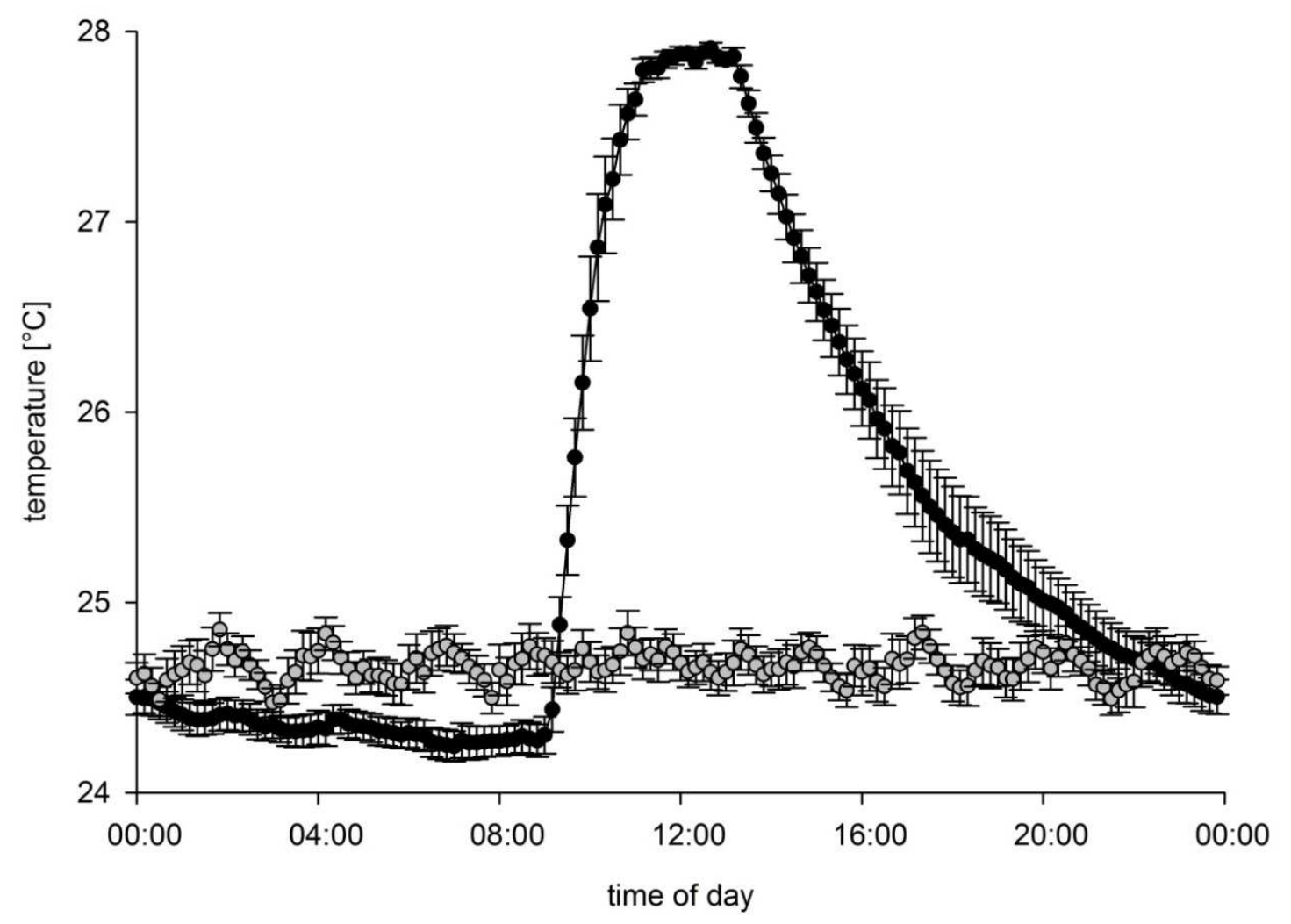

Figure 8.1. Acclimation treatments used for Symbiodinium B1.

Daily temperature ranges for the constant (grey) and fluctuating (black) acclimation treatment as interpolated scatter plots. Values represent means $\pm 95 \% \mathrm{Cl}$ of 32 days of acclimation. 
Table 8.1. Statistical analysis for acclimation experiment.

Within-subject effects of the rmANOVA are listed. Significant effects at $p<0.05$ are indicated by an asterisk.

\begin{tabular}{|r|r|r|r|r|}
\hline Variable & \multicolumn{1}{|c|}{ Time } & \multicolumn{1}{c|}{$\begin{array}{r}\text { Time } \mathrm{x} \\
\text { Temperature }\end{array}$} & $\begin{array}{r}\text { Time } \times \text { Acclimation } \\
\text { mode }\end{array}$ & $\begin{array}{r}\text { Time } \mathrm{x} \\
\text { Temperature } \mathrm{x} \\
\text { Acclimation mode }\end{array}$ \\
\hline $\mathrm{F}_{\mathrm{v}} / \mathrm{F}_{\mathrm{m}}$ & $\mathrm{F}_{2,11}=6.3809$, & $\mathrm{F}_{2,11}=225.9159$, & $\mathrm{F}_{2,11}=1.1397$, & $\mathrm{F}_{2,11}=20.5630$, \\
$\mathrm{p}=0.0145^{*}$ & $\mathrm{p}<0.0001^{*}$ & $\mathrm{p}=0.3550$ & $\mathrm{p}=0.0002^{*}$ \\
\hline Chl a & $\mathrm{F}_{2,11}=4.4628$, & $\mathrm{F}_{2,11}=0.57$, & $\mathrm{F}_{2,11}=0.6525$, & $\mathrm{F}_{2,11}=0.3617$, \\
content & $\mathrm{p}=0.0381^{*}$ & $\mathrm{p}=0.5814$ & $\mathrm{p}=0.5398$ & $\mathrm{p}=0.7045$ \\
\hline Cell & $\mathrm{F}_{2,11}=422.9798$, & $\mathrm{F}_{2,11}=206.3351$, & $\mathrm{F}_{2,11}=0.6043$, & $\mathrm{F}_{2,11}=0.9674$, \\
density & $\mathrm{p}<0.0001^{*}$ & $\mathrm{p}<0.0001^{*}$ & $\mathrm{p}=0.5636$ & $\mathrm{p}=0.4102$ \\
\hline Protein & $\mathrm{F}_{2,11}=20.4219$, & $\mathrm{F}_{2,11}=9.9318$, & $\mathrm{F}_{2,11}=0.29$, & $\mathrm{F}_{2,11}=0.7427$, \\
content & $\mathrm{p}=0.0002^{*}$ & $\mathrm{p}=0.0034^{*}$ & $\mathrm{p}=0.7538$ & $\mathrm{p}=0.4983$ \\
\hline SOD & $\mathrm{F}_{2,11}=22.9868$, & $\mathrm{F}_{2,11}=28.8713$, & $\mathrm{F}_{2,11}=0.1939$, & $\mathrm{F}_{2,11}=0.0696$, \\
& $\mathrm{p}=0.0001^{*}$ & $\mathrm{p}<0.0001^{*}$ & $\mathrm{p}=0.8265$ & $\mathrm{p}=0.9332$ \\
\hline APX & $\mathrm{F}_{2,11}=4.6381$, & $\mathrm{F}_{2,11}=30.8692$, & $\mathrm{F}_{2,11}=0.3033$, & $\mathrm{F}_{2,11}=0.1568$, \\
& $\mathrm{p}=0.0346^{*}$ & $\mathrm{p}<0.0001^{*}$ & $\mathrm{p}=0.7444$ & $\mathrm{p}=0.8568$ \\
\hline
\end{tabular}



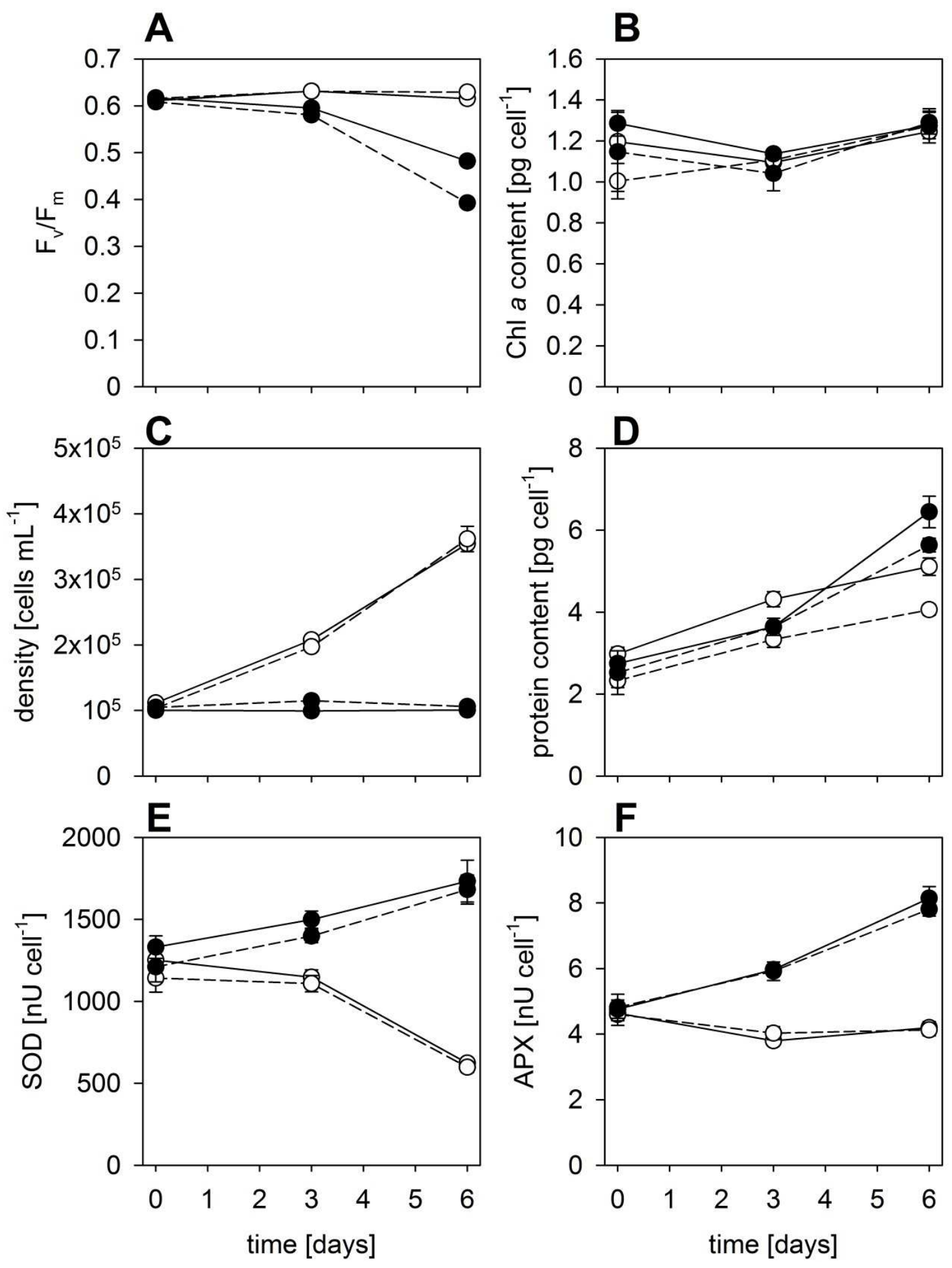

Figure 8.2. Effects of thermal acclimation mode on physiological variables under thermal stress in Symbiodinium B1.

Response of maximum quantum yield $(A)$, chlorophyll a content $(B)$, growth $(C)$, protein content (D), superoxide dismutase activity (E), and ascorbate peroxidase (D) in Symbiodinium $\mathrm{B} 1$ at $25^{\circ} \mathrm{C}$ (white) and $33^{\circ} \mathrm{C}$ (black) shown as interpolated scatter plots. Cultures were acclimated to constant $\left(25^{\circ} \mathrm{C}\right.$, straight line) or fluctuating temperatures $\left(25-28^{\circ} \mathrm{C}\right.$, dashed line) prior to the thermal exposure. Values are means $\pm S E(N=4)$. 